\title{
Induction, Bounding, Weak Combinatorial Principles, and The Homogeneous Model Theorem
}

\author{
Denis R. Hirschfeldt \\ Karen Lange \\ Richard A. Shore
}

\author{
Author address: \\ Department of Mathematics, University of Chicago, 5734 S. Uni- \\ Versity Ave., Chicago, IL 60637 \\ E-mail address: drh@math.uchicago.edu \\ Department of Mathematics, Wellesley College, 106 Central St., \\ Wellesley, MA 02481 \\ E-mail address: klange2@wellesley.edu \\ Department of Mathematics, Malott Hall, Cornell University, \\ ITHACA, NY 14853 \\ E-mail address: shore@math.cornell.edu
}





\section{Contents}

Chapter 1. Introduction 1

1.1. The thematic level 1

1.2. The specifics 5

1.3. An outline of the paper 13

Chapter 2. Definitions 15

2.1. Reverse mathematics 15

2.2. Model theoretic notions 16

2.3. Other notions 19

Chapter 3. The Atomic Model Theorem and Related Principles 21

Chapter 4. Defining Homogeneity 27

Chapter 5. Closure Conditions and Model Existence $\quad 41$

5.1. Type spectra of homogeneous models 41

5.2. Type spectra of general models 43

5.3. Comparing closure conditions 44

5.4. Spectrum enumeration existence theorems 45

5.5. Model existence theorems 48

Chapter 6. Extension Functions and Model Existence $\quad 51$

6.1. Extension functions 51

6.2. The reverse mathematics of extension functions 56

Chapter 7. The Reverse Mathematics of Model Existence Theorems $\quad 75$

7.1. Extension function approximations, AMT, and ATT 76

7.2. Comparing model existence theorems 83

7.3. Computability theoretic equivalences 84

$\begin{array}{lll}\text { Chapter 8. } & \text { Open Questions } & 87\end{array}$

Appendix A. Approximating Generics $\quad 89$

Appendix B. Atomic Trees 93

$\begin{array}{lll}\text { Appendix C. } & \text { Saturated Models } & 97\end{array}$

$\begin{array}{ll}\text { Bibliography } & 99\end{array}$ 



\begin{abstract}
Goncharov and Peretyat'kin independently gave necessary and sufficient conditions for when a set of types of a complete theory $T$ is the type spectrum of some homogeneous model of $T$. Their result can be stated as a principle of second order arithmetic, which we call the Homogeneous Model Theorem (HMT), and analyzed from the points of view of computability theory and reverse mathematics. Previous computability theoretic results by Lange suggested a close connection between HMT and the Atomic Model Theorem (AMT), which states that every complete atomic theory has an atomic model. We show that HMT and AMT are indeed equivalent in the sense of reverse mathematics, as well as in a strong computability theoretic sense. We do the same for an analogous result of Peretyat'kin giving necessary and sufficient conditions for when a set of types is the type spectrum of some model.

Along the way, we analyze a number of related principles. Some of these turn out to fall into well-known reverse mathematical classes, such as $\mathrm{ACA}_{0}, \mathrm{I} \Sigma_{2}^{0}$, and $\mathrm{B} \Sigma_{2}^{0}$. Others, however, exhibit complex interactions with first order induction and bounding principles. In particular, we isolate several principles that are provable from I $\Sigma_{2}^{0}$, are (more than) arithmetically conservative over $\mathrm{RCA}_{0}$, and imply I $\Sigma_{2}^{0}$ over $\mathrm{B} \Sigma_{2}^{0}$. In an attempt to capture the combinatorics of this class of principles, we introduce the principle $\Pi_{1}^{0} \mathrm{GA}$, as well as its generalization $\Pi_{n}^{0} \mathrm{GA}$, which is conservative over $\mathrm{RCA}_{0}$ and equivalent to $\mathrm{I} \Sigma_{n+1}^{0}$ over $\mathrm{B} \Sigma_{n+1}^{0}$.
\end{abstract}

Received by the editor July 22, 2015.

2010 Mathematics Subject Classification. Primary 03B30; Secondary 03C07, 03C15, 03C50, 03C57, 03D45, 03F30, 03F35.

Hirschfeldt was partially supported by the National Science Foundation of the United States, grants DMS-0801033 and DMS-1101458.

Lange was partially supported by NSF Grants DMS-0802961 and DMS-1100604.

Shore was partially supported by NSF Grants DMS-0554855, DMS-0852811, and DMS1161175, John Templeton Foundation Grant 13408, and a short term visiting position at the University of Chicago as part of the Mathematics Department's visitor program. 



\section{CHAPTER 1}

\section{Introduction}

This paper began as an investigation into the difficulty (in terms of the axioms needed) of proving certain theorems of classical model theory about the existence of models of given theories with specific properties (in particular homogeneity). We were also motivated by, and interested in illuminating, the relations of these proof theoretic analyses with ones of the computational complexity of constructing these models. In analyzing these questions we were led into several byways: proof theoretic, computational, and combinatorial. The paper has thus become a case study in reverse mathematics and its relations to effective or computable mathematics.

We begin our introduction with a thematic explanation of the format and goals of reverse mathematics as well as its connections in general to computable mathematics. We then describe the nature of our results and the places that they occupy within reverse mathematics on the same thematic level. After discussing some previous related results (both reverse mathematical and computability theoretic), we give a more specific, even formal, description of the questions considered and the results provided. Finally, we provide an outline of the full paper in Section 1.3.

\subsection{The thematic level}

The general goal of reverse mathematics is to calibrate the complexity of mathematical theorems by determining precisely the systems of axioms needed to prove the given theorems. More specifically, the subject deals with what might be called countable mathematics in that usually it restricts its attention to theorems about countable structures (algebraic, logical, or combinatorial) or ones in the parts of analysis and topology (such as separable metric spaces) that can be approximated by countable sets. (For some approaches to the uncountable see [25] and [45].)

One direction of this calibration is standard. We know what it means for a theorem to be provable from a set of axioms. The other direction provides both the preciseness of the answers and the name of the subject. We reverse the usual situation by proving the axioms from the theorem. Formally, this means starting with some common weak base set of axioms $B$ and an axiom system $S$ extending $B$ that proves the theorem of interest, $\Phi$. Then one establishes a reversal by showing that $B \cup\{\Phi\}$ proves $S$. Thus, relative to the weak base theory $B$, the set of axioms $S$ proving $\Phi$ and $\Phi$ itself are logically equivalent. So the proof theoretic, or better reverse mathematical, strength of $\Phi$ is determined to be precisely that of $S$.

Given that our subjects of interest are countable, the usual and natural setting for reverse mathematics is second order arithmetic (rather than say set theory). The language here is that of ordinary first order arithmetic $(+, \times, \leqslant, 0$, and 1$)$ with first order logic (including the usual variables $x, y, z, \ldots$ ranging over "the numbers") supplemented by a collection of distinct variables $X, Y, Z \ldots$ (and perhaps constant symbols $A, B, \ldots$ ) ranging over (or denoting specific) "sets of numbers" 
along with a binary relation symbol $x \in X$ representing membership of numbers $x$ in sets $X$. The axioms for a system of second order arithmetic begin with the standard basic ones for arithmetic with,$+ \times, \leqslant, 0$, and 1 and an induction principle saying that every set containing 0 and closed under successor contains all the numbers (or, equivalently, that every nonempty set has a least element). It may then be augmented by additional comprehension axioms asserting that sets with some properties (e.g. definable by formulas in some given class) exist. One may also add axioms asserting induction principles of the form that if 0 has some property (e.g. as specified by a formula of some class) and the numbers with this property are closed under successor then every number has the specified property (or the corresponding least number principle as above). We give more precise definitions and examples in Section 2.1.

There is a close connection between reverse and effective mathematics and between the standard axiomatic systems of reverse mathematics and the calibration schemes from computability theory for the complexity of sets and functions. To make this correspondence clear (and for many other purposes), we need to specify the semantics for axiom systems for second order arithmetic. A structure for this language is one of the form $\mathcal{M}=\langle M, S,+, \times, \leqslant, 0,1, \in\rangle$ where $M$ is a set (the set of the "numbers" of $\mathcal{M}$ ) over which the first order quantifiers and variables of our language range; $S \subseteq 2^{M}$ is the collection of subsets of the "numbers" in $\mathcal{M}$ over which the second order quantifiers and variables of our language range; as usual, + and $\times$ are binary functions on $M$; and $\leqslant$ is a binary relation on $M$ while 0 and 1 are members of $M$. We always interpret $\epsilon$ as the usual membership relation between elements of $M$ and elements of $S$.

The standard weak base system for reverse mathematics, $\mathrm{RCA}_{0}$, contains, in addition to the usual basic axioms for arithmetic: $\mathrm{I} \Sigma_{1}^{0}$, induction for $\Sigma_{1}^{0}$ formulas (i.e., ones with one (or a block of) existential quantifier(s) followed by a quantifier free matrix); and $\Delta_{1}^{0}-\mathrm{CA}_{0}$, comprehension for sets determined by equivalent $\Sigma_{1}^{0}$ and $\Pi_{1}^{0}$ formulas (the latter being ones with one universal quantifier or block of universal quantifiers). This system corresponds to computable (or recursive) mathematics in the sense that the models of this theory whose numbers $M$ are just the usual natural numbers $\mathbb{N}$ are precisely the ones whose sets are closed under join (i.e., effective union) and Turing reducibility; i.e., if $A, B \in M$ then any set computable from the pair $A \oplus B$ is also in $M$. (The structures $\mathcal{M}$ with $M=\mathbb{N}$ are called $\omega$-models.) Thus the theorems of $\mathrm{RCA}_{0}$ are essentially theorems of computable mathematics and the converse usually holds as well except at times when the classical computability theory style proofs rely on more than $\Sigma_{1}^{0}$ induction. In this paper we also can often follow proofs from computable model theory to derive their analogs in $\mathrm{RCA}_{0}$. More frequently, though, $\mathrm{RCA}_{0}$ does not suffice.

Reverse mathematics had its beginnings in the work of Harvey Friedman in the late '60s and early '70s $([\mathbf{1 1}, \mathbf{1 2}, \mathbf{1 3}])$. Its early development is well chronicled in Simpson's classic text [46], which is also the source for many important ideas and results. It has four basic systems in addition to $\mathrm{RCA}_{0}$, and the $\omega$-models of each correspond in a similar way to closure conditions on their sets familiar from computability theory. The primary story of the first few decades of reverse mathematics was that almost every standard theorem from the literature of classical mathematics that was analyzed was equivalent over $\mathrm{RCA}_{0}$ (in the sense of reverse mathematics described above) to one of these five systems. They were thus dubbed the "big five". 
Moreover, each of these systems corresponds to a well known philosophical system for mathematics as well as to a standard level of complexity in computability theory. (For details, here and elsewhere, as well as a thorough general introduction to reverse mathematics, we refer the reader to the second edition of the standard text by Simpson [47]. For a shorter introduction focused on combinatorial and model theoretic principles, see Hirschfeldt [20]. A very brief introduction can be found in Shore [44].)

In addition to $\mathrm{RCA}_{0}$, the two next stronger of the big five are the ones most relevant to this paper: $\mathrm{ACA}_{0}$ and $\mathrm{WKL}_{0}$. The first extends $\mathrm{RCA}_{0}$ by adding on $\Sigma_{n}^{0}-\mathrm{CA}_{0}$ for every $n \in \mathbb{N}$, i.e., arithmetic comprehension: all sets defined by formulas of first order arithmetic (with set parameters) exist. It corresponds to closure under the Turing jump (i.e., the halting problem for machines with oracles in the structure). (The Turing jump and its iterates provide the most common measure of complexity in computability theory.) The second (and weaker) system corresponds to structures in which the collection of sets forms what is called a Scott set; i.e., every infinite binary tree in the collection has an infinite path in it as well. Each of these three systems has many equivalents in most areas of mathematics. Following the classical story of reverse mathematics we provide more equivalences in this paper.

On the other hand, a very interesting and, in our view, important trend in reverse mathematics has emerged in the past decade or so. Many theorems of standard mathematics have been found to lie outside the scope of the big five systems. Some are stronger than all of them, some are weaker even than $\mathrm{WKL}_{0}$, and some are incomparable with $\mathrm{WKL}_{0}$ but still below $\mathrm{ACA}_{0}$. Starting with papers such as [5], [22], and [23], combinatorics and model theory have been primary sources of such examples. Earlier examples can be found in papers like [42], but these more recent papers began a trend of exploring the reverse mathematics of large classes of related combinatorial and model theoretic principles not in the scope of the big five. (Expositions and discussions of these phenomena can be found in [44] and much more extensively in [20].) In this paper we continue along this road with new examples from model theory of theorems with comprehension strength lying outside the big five.

A yet newer phenomenon in reverse mathematics is the appearance of a large number of examples of principles escaping the standard systems because of issues that lie along the induction rather than (or in addition to) the comprehension spectrum. One striking example at the high end of the standard systems is provided by work of Montalbán [32] and Neeman $[\mathbf{3 8}, \mathbf{3 9}]$. In the last of these three papers, Neeman showed that in the proof of an equivalence in the second paper (sharpening results of the first paper), the use of $\mathrm{I} \Sigma_{1}^{1}$ (induction for formulas with leading second order existential quantifiers followed by a first order matrix) cannot be eliminated. Another example where high levels of induction are required is in Montalbán and Shore [35]. They prove a result in $\mathrm{ATR}_{0}$ (the system from the big five after $\mathrm{ACA}_{0}$ ) using transfinite induction. In particular, they use induction on arbitrary well orderings (instead of just of $\mathbb{N}$ ) for classes defined by $\Pi_{1}^{1}$ formulas (so second order with one leading universal quantifier). However, the theorem fails without the extra induction assumption.

In this paper, in addition to cases where extra comprehension axioms are needed, we often run into issues at the lowest levels of the induction spectrum. 
Indeed, this happens first at the stage of choosing the "right" definitions. In our case, there is one important variation of homogeneity that requires comprehension axioms $\left(\mathrm{ACA}_{0}\right)$ to prove its equivalence to the others (Proposition 4.2). This equivalence is thus better viewed as a theorem and, indeed, is usually cited as such in the literature. On the other hand, there are a number of variations on the standard definitions that are used interchangeably and not distinguished in either the classical or computability theoretic literature. These variations are ones for which the usual proofs of equivalence use different levels of induction-like axioms. Here, we show reversals, in that the equivalences imply the axioms used to prove them over $\mathrm{RCA}_{0}$. (Examples include Theorems 4.3 and 4.4.) Which definition is chosen can play a crucial role in getting the "right" theorem characterizing the strength of our model theoretic principles.

In addition, even with the "right" choice of definition, more induction than is available in $\mathrm{RCA}_{0}$ is used in the classical or computability theoretic proofs at a number of crucial junctures (e.g. for $\Sigma_{2}^{0}$ rather than $\Sigma_{1}^{0}$ formulas). Here, three routes are followed for different theorems. One applies the method of Shore blocking from generalized recursion theory $([\mathbf{4 3}])$ to carry out, in $\mathrm{RCA}_{0}$, arguments that seem to need $\mathrm{I} \Sigma_{2}^{0}$. (Examples include Theorem 6.10 as well as Theorem 4.1 of [23].) The second provides reversals to show that the theorems of interest are reverse mathematically equivalent to some induction-like principle. (Examples are Theorem 4.3 for $\mathrm{I} \Sigma_{2}^{0}$ and Theorem 4.4 for $\mathrm{B} \Sigma_{2}^{0}$, the bounding principle for $\Sigma_{2}^{0}$ formulas, which will be defined in Section 2.1 and has been shown by Slaman [48] to be equivalent to $\Delta_{2}^{0}$-induction.) The third, and most interesting, route is one that leads to new axiomatic systems, theorems, and combinatorial principles of strength intermediate among, or incomparable with, the standard hierarchy of induction type axioms (which includes the usual bounding axioms). The driving examples include entries labeled 3 or 4 in Table 6.1 on page 57 that lead to the combinatorial principle $\Pi_{1}^{0}$ GA used extensively in Chapter 6. This principle is generalized in Appendix A to principles $\Pi_{n}^{0} \mathrm{GA}$ that fit into all levels of the induction hierarchy in a most unusual way. Each follows from the next level of induction: $\mathrm{RCA}_{0}+\mathrm{I}_{n+1}^{0} \vdash \Pi_{n}^{0} \mathrm{GA}$. However, $\Pi_{n}^{0} \mathrm{GA}$ is strictly weaker in two ways. First, even $\mathrm{B} \Sigma_{2}^{0}$ does not follow from any $\Pi_{n}^{0} \mathrm{GA}$. Second, for every $n$, we have $\mathrm{RCA}_{0} \vdash \Pi_{n}^{0} \mathrm{GA}+\mathrm{B} \Sigma_{n+1}^{0} \leftrightarrow \mathrm{I} \Sigma_{n+1}^{0}$.

A foundationally challenging phenomenon arising in this paper is provided by theorems or constructions that have essentially two provably different proofs. In particular, we have theorems of model theory that are provable from, for example, either $\mathrm{WKL}_{0}$ or $\mathrm{I} \Sigma_{2}^{0}$ and others from $\mathrm{WKL}_{0} \vee \mathrm{B} \Sigma_{2}^{0}$ (as described, e.g., following Proposition 4.5) or the disjunction of other pairs of comprehension and induction type axioms (see, e.g., the remarks preceding Question 8.5 and items labeled 3 or 4 in Table 6.1). When such a principle is not provable in $\mathrm{RCA}_{0}$, there may not be any canonical "best" proof or axiomatic system for it. The only earlier example of this phenomenon of which we are aware is the fact that the principle that iterations of continuous functions are continuous is equivalent to $\mathrm{WKL}_{0} \vee \mathrm{I} \Sigma_{2}^{0}$ (Friedman, Simpson, and $\mathrm{Yu}[\mathbf{1 4}]$ ). Many additional examples of this phenomenon and related ones can be found in recent work of Belanger $[\mathbf{3}]$. (Some of his equivalents for $\mathrm{WKL}_{0} \vee \mathrm{I} \Sigma_{2}^{0}$ in terms of amalgamation of types are mentioned in Theorem 5.12 below.) An interesting question then is what is the impact of such results on the philosophical or foundational program of reverse mathematics? (A different 
foundational question is posed by other results by Belanger $([\mathbf{2}, \mathbf{3}])$ where model theoretic facts are shown to be equivalent to $\mathrm{ACA}_{0} \vee \neg \mathrm{WKL}_{0}$.)

\subsection{The specifics}

In order to describe the actual problems analyzed in our results, we require specific formal definitions of the standard axiomatic systems being used (and some variations on them) as well as the model theoretic notions being analyzed in both the computability theoretic and reverse mathematical settings. These are provided in Chapter 2. Here we give a description for the reader who already has a basic familiarity with reverse mathematics and elementary model theory or who will refer to Chapter 2 as needed. For now we just note that all languages (and so theories) and structures are assumed to be countable. Additionally, we assume all theories to be complete and consistent. So in the setting of computable model theory, for us, the notions of computable and decidable coincide for theories. In the setting of reverse mathematics, we include the full elementary diagram in the presentation of a structure. (From the viewpoint of effective model theory then, we study only decidable structures for which the full elementary diagram is computable rather than computable structures for which only the atomic diagram is assumed to be computable.)

The initial motivation for our study was the investigation by Hirschfeldt, Shore, and Slaman of the reverse mathematical complexity of several classical model theoretic theorems in $[\mathbf{2 3}]$ and related work from the viewpoint of computability theory that both preceded and followed it, in particular the analysis of what in [23] is called AMT, the Atomic Model Theorem: every atomic theory has an atomic model.

Harrington [19] and independently Goncharov and Nurtazin [16] proved an important early result on atomic models: An atomic, (complete) decidable theory $T$ has a decidable atomic model if and only if there exists a uniformly computable listing of all of the principal types of $T$, i.e., all the types realized in the atomic model. From the relativized version of the above characterization, it easily follows that every atomic, decidable theory has a $\mathbf{0}^{\prime}$-decidable atomic model, i.e., one whose full elementary diagram is computable in $\mathbf{0}^{\prime}$. Csima [7] greatly improved this result by showing that such a theory always has an atomic model decidable in some low degree. Csima, Hirschfeldt, Knight, and Soare [9] studied atomic bounding degrees, where a degree $\mathbf{d}$ is atomic bounding if every atomic, decidable theory has a $\mathbf{d}$-decidable atomic model. They showed that the $\Delta_{2}^{0}$ atomic bounding degrees $\mathbf{x}$ (i.e., $\mathbf{x} \leqslant_{\mathrm{T}} \mathbf{0}^{\prime}$ ) are exactly the nonlow 2 ones (i.e., $\mathbf{x}^{\prime \prime}>_{\mathrm{T}} \mathbf{0}^{\prime \prime}$ ). (These computability theoretic papers used the word "prime" in place of "atomic". The two notions are classically equivalent (for countable models) but differ in our context, as discussed extensively in $[\mathbf{2 3}]$ and a bit in this paper.) The question of which degrees not below $\mathbf{0}^{\prime}$ are atomic bounding is more complicated (see Conidis [6]) but is connected in various ways to the reverse mathematical strength of AMT as analyzed in [23].

In [23], it is shown that AMT is not provable in $\mathrm{RCA}_{0}$, is incomparable with $\mathrm{WKL}_{0}$, and is quite a weak theory. Indeed, it is strictly weaker than all the combinatorial principles considered by Hirschfeldt and Shore $[\mathbf{2 2}]$ that are not $\Pi_{1}^{1}$ conservative over $\mathrm{RCA}_{0}$. It is also shown in $[\mathbf{2 3}]$ that $\mathrm{AMT}$ is more than $\Pi_{1}^{1}$ conservative over $\mathrm{RCA}_{0}$ and is $\Pi_{1}^{1}$-conservative over $\left(\mathrm{RCA}_{0}+\right) \mathrm{B} \Sigma_{2}^{0}$. (A system of axioms $S$ is $\Gamma$-conservative over another system $T$ (for $\Gamma$ a class of formulas) if for 
any $\Phi \in \Gamma$, if $S+T \vdash \Phi$ then $T \vdash \Phi$. So this is a way of saying that $S+T$ is not stronger than $T$ at least as far as theorems in $\Gamma$ are concerned.)

Since classically every (countable) atomic model is homogeneous, it is natural to study how degree theoretic and reverse mathematical results on homogeneous models compare with ones on atomic models. Now there are two ways to view the possible generalization of AMT from atomic to homogeneous models. The first views atomic models as ones whose elements satisfy a particular property. (They each realize some atom, i.e., a formula generating a principal type.) The second characterizes the atomic models by their type spectra, the sets of types they realize. (They realize precisely the principal types.) Since these characterizations are classically equivalent, the notion of atomic bounding degree captures the question of how hard it is to build an atomic model of any decidable atomic theory and is equivalent to the question of how hard it is to construct a model that realizes precisely the set of principal types of such a theory. These questions then both reasonably correspond to AMT in reverse mathematics.

For homogeneous models the two views are different. The first asks the simpler question of how hard it is to build a homogeneous model of any given decidable theory. Here the degree theoretic answer given by Csima, Harizanov, Hirschfeldt, and Soare $[\mathbf{8}]$ is that it is exactly the PA degrees (the ones of complete extensions of Peano arithmetic or equivalently of Scott sets) that suffice for these constructions. As would then be expected, the reverse mathematical analog is that the existence of a homogeneous model for every theory is equivalent to $\mathrm{WKL}_{0}$ (Lange $[\mathbf{2 6}]$; see also Belanger $[\mathbf{3}]$ ). This is also the computational and reverse mathematical complexity of being able to produce a completion of any (computable) consistent set $S$ of sentences, and hence a model of $S$ (see [47, Theorem IV.3.3]).

In this paper, we are concerned with the more difficult question of determining when is there a homogeneous model with a given type spectrum. (It is worth mentioning that classically a homogeneous model is determined up to isomorphism by its type spectrum; see Proposition 4.7 for a reverse mathematical analysis of this fact.) The first question, even classically, is then which sets of types are type spectra of homogeneous models. Consider the situation in computable model theory. Clearly, for any decidable model, there is a computable enumeration of the types it realizes. Goncharov [15] and Peretyat'kin [41] independently showed that there are additional conditions on a computable listing of types $X$ that are necessary and sufficient for there to be a decidable homogeneous model $\mathcal{A}$ such that $X$ is an enumeration of the type spectrum of $\mathcal{A}$. Classically, it is easy to see that the set of types realized in a homogeneous model must satisfy certain amalgamation properties for formulas and types. To get a decidable homogeneous model, an additional effectiveness condition is needed on the listing of types. It must have what we call a computable extension function approximation that indicates (in terms of their indices) how types and formulas can be amalgamated. (See Section 6.1 for a formal definition.) The results of Goncharov and Peretyat'kin also answer the question classically if one ignores the computability theoretic restrictions and simply requires that the set of types be closed under the appropriate amalgamation procedures (see Theorem 5.1).

In $[\mathbf{2 7}]$ and $[\mathbf{2 8}]$, Lange studied the degree theoretic analog of the second view of AMT for homogeneous models. She showed that for any computable list of types $X$ with the appropriate amalgamation properties, there is a model $\mathcal{A}$ decidable in 
some low degree such that $X$ is an enumeration of the type spectrum of $\mathcal{A}$. She also investigated the question of which degrees $\mathbf{d}$ have the property that, given any $X$ as above, there is a $\mathbf{d}$-decidable homogeneous model $\mathcal{A}$ such that $X$ is an enumeration of the type spectrum of $\mathcal{A}$. These we might call the homogeneous bounding degrees. (Lange and Soare $[\mathbf{2 9}]$ called them the $\mathbf{0}$-bounding degrees. Note that the papers $[\mathbf{8}$, $\mathbf{9}, \mathbf{2 9}$ ] use the term "homogeneous bounding degrees" in the other sense mentioned above, where the class of such degrees coincides with that of the PA degrees.) Lange showed that, as for atomic models, the homogeneous bounding degrees $\mathbf{d} \leqslant \mathbf{0}^{\prime}$ are precisely the $\mathbf{d}$ with $\mathbf{d}^{\prime \prime}>\mathbf{0}^{\prime \prime}$. Comparing these degree theoretic results (and others) with the aforementioned ones of Csima [7] and Csima, Hirschfeldt, Knight, and Soare [9] for atomic models suggested a deeper connection between model existence principles for atomic and homogeneous models. However, the proofs of these results for homogeneous models and for atomic models seemed quite different. In this kind of situation, where analogies between disparate principles suggest but do not immediately provide a precise connection, reverse mathematical and computability theoretic analysis can often clarify the situation. In this paper, we show that the model existence principle studied by Lange and AMT are indeed connected in a strong way, as seen from the viewpoints of reverse mathematics and computability theory. In particular, our results explain the similarity between Lange's results for homogeneous models and the ones for atomic models by showing that, in a precise sense, both sets of results are dealing with the same principle, in two different guises.

First, we must provide an analog to the principle AMT. Recall that the view of AMT that we are trying to emulate states that every atomic theory $T$ has a model whose type spectrum consists of the principal types of $T$. In the homogeneous case, we want a principle, the Homogeneous Model Theorem (HMT), asserting that if a list of types could be an enumeration of the type spectrum of a homogeneous model, then there in fact exists a homogeneous model whose type spectrum is enumerated by this list. The characterization of such lists of types in the computability theoretic setting in [15] and [41] mentioned above provides us with the obvious starting point. In Section 5.1, we present several variations on the conditions given in these characterizations that are classically equivalent but differ when viewed reverse mathematically. In Chapter 4, we give several definitions of homogeneity that are also classically equivalent, but differ in the setting of reverse mathematics. We obtain three versions of the HMT principle depending on how we describe the closure conditions on the list of types and which definition of homogeneity we use. In Section 7.2, we show that these versions of HMT imply AMT and that AMT implies one of them over $\mathrm{RCA}_{0}$ (and the other ones with sufficient additional induction assumptions). Thus the "right" versions supply us with the theorem on the existence of homogeneous models with specified type spectra that is reverse mathematically equivalent to the atomic model theorem.

Our proofs of these reverse mathematical results actually provide degree theoretic information that greatly strengthens the computational analogy between atomic and homogeneous models (from the second viewpoint above). Indeed, we show (Corollary 7.12) that the atomic bounding degrees are precisely what we have called the homogeneous bounding degrees.

At this point, we also note that while we were originally motivated by the idea of the analogy between existence principles for atomic and homogeneous models, and 
the analogy proved precise for the second view of AMT, there is also a close analogy between these principles and existence principles for arbitrary models. As we mentioned above, the pure model existence problem for sets of consistent sentences is reverse mathematically (and degree theoretically) equivalent to $\mathrm{WKL}_{0}$ (the $\mathrm{PA}$ degrees), as is the existence problem for homogeneous models of theories (or consistent sets of sentences). If we move to the second view of AMT, where we require the existence of a model with any given "possible" type spectrum, and move from arbitrary consistent sets of sentences to theories, our analysis shows that the existence of arbitrary models, AMT, and HMT are both reverse mathematically (with the "right" definitions or induction assumptions) and degree theoretically equivalent. (Notice that specifying a set of types automatically specifies a (complete) theory.)

Of course, one needs the appropriate necessary and sufficient conditions for a set of types to be the type spectrum of an arbitrary model. These are given in [41] for both the classical and computability theoretic settings. In Theorem 5.7 we present these conditions, and in the following sections we carry out the analysis for arbitrary models along with the one for homogeneous models. The outcome of this analysis is that one of the variants (WMT) of the existence theorem for arbitrary models is reverse mathematically equivalent to HMT (Theorem 7.6). Moreover, the analogously defined class of model bounding degrees is the same as the classes of atomic and homogeneous bounding degrees (Corollary 7.12). As with HMT there are variants for arbitrary models that are classically and computably equivalent but may differ depending on the types of induction-like axioms available. Before turning to these questions, we note that not only are AMT, HMT, and WMT equivalent over $\mathrm{RCA}_{0}$ but, if we work over $\mathrm{I} \Sigma_{2}^{0}$, they are also equivalent to all the variants that we are about to discuss, as well as the combinatorial principles $\Pi_{1}^{0} \mathrm{G}$ and ATT that we consider below. Thus AMT is a candidate for a system that, like each of the big five, is what Montalbán ([33] and [34]) has called robust, a term that still lacks a precise definition but is informally defined in $[\mathbf{3 4}]$ as "equivalent to small perturbations of itself". Another example of a robust system outside the big five is $\mathrm{WWKL}_{0}$ (see [47, Section X.1] for a definition of this system).

As we mentioned above, issues connected to induction arise already when we try to formalize the definition of homogeneous model in Chapter 4. The usual classical definition requires that, given two sequences of the same type and an extension of one of them, we can find a corresponding extension of the other such that the extended sequences continue to have the same type. One can vary this idea by considering extensions of length 1 or of arbitrary finite length, and also by requiring that extensions can be found for finitely many pairs of given sequences as in Definition 4.1(i)-(iii). All these variants are classically and computably equivalent since the proofs of equivalence require only simple induction arguments. These proofs are not quite simple enough, however, to work in $\mathrm{RCA}_{0}$. There is one additional standard version (Definition 4.1(iv)), which we call strong 1-homogeneity, that requires the existence of an automorphism taking one of the two given sequences with the same type to the other. This is "obviously" a much stronger condition. The usual proof is not computable and seems to require the jump of the model to carry it out. Not surprisingly, we can show that this level of complexity is computationally and reverse mathematically necessary. The equivalence of this characterization of homogeneity to any of the others is itself reverse mathematically equivalent to $\mathrm{ACA}_{0}$ (Proposition 4.2). On the other hand, given this result, it is surprising to 
note that the existence theorem that every theory has a homogeneous model in this strong sense remains at the level of $\mathrm{WKL}_{0}$ (Lange [26] and Belanger [3]).

The analysis of the relations among the other variations on homogeneity (Chapter 4) are more interesting and unusual, as are the ones (Chapter 5) of the variations on the amalgamation closure conditions in the definition of the "possible" sets of types that could be type spectra of homogeneous models. These two sets of variations are corresponding ones, in that the closure conditions are the ones that obviously hold for models satisfying the corresponding notion of homogeneity. (Thus there is a match up for possible candidates for the "right theorem".) The analyses of each set of variations and the relations between them provide reverse mathematical equivalents to induction type axioms: $\mathrm{I} \Sigma_{2}^{0}$ (Theorems $4.3,5.11$, and 5.14 and the entries labeled 2 in Table 6.1) and $\mathrm{B} \Sigma_{2}^{0}$ (Theorems 4.4, 5.15, and 5.16).

Now there are many logical, computability theoretic, and combinatorial equivalents of the standard versions of these induction type axioms at all levels of their hierarchies $\left(\mathrm{I} \Sigma_{n}^{0}\right.$ and $\left.\mathrm{B} \Sigma_{n}^{0}\right)$. Some can be found, for example, in the standard (and quite comprehensive) text on first order arithmetic by Hájek and Pudlák [17, Section A.I.2]. Others in the setting of second order arithmetic are scattered throughout the reverse mathematical literature. Our results here, however, are about model theoretic properties. Their proofs are often given by delicate and at times complicated constructions of theories and specific models. The models constructed are designed to satisfy one variant of homogeneity but such that, if they satisfied another, we could prove one of the standard equivalents of $\mathrm{I} \Sigma_{2}^{0}$ or $\mathrm{B} \Sigma_{2}^{0}$.

Much more unusual and surprising are the roles that induction plays in the analysis of the construction of some of the variations of homogeneous models. Here, we want to construct a model with a suitable given type spectrum. Of course, we are assuming that the potential type spectrum satisfies some version of the conditions required for there to be such a model in the computability theoretic setting. (As mentioned above, these conditions were given by both Goncharov [15] and Peretyat'kin [41] independently.) Examples of such constructions include Theorems 5.1 and 6.4. In particular, we assume that there is an "extension function approximation" (Definition 6.2) that approximates the action (in terms of indices) on the given list of types that the amalgamation relations require. Here we refer the reader especially to the entries marked 3 of Table 6.1 on page 57 .

The ones in the column labeled "pairwise full amalgamation" are all construction principles for homogeneous or arbitrary models with given type spectra of the appropriate kinds. (The pairwise full amalgamation closure conditions form one of our versions of the Goncharov-Peretyat'kin conditions mentioned above.) Our original proofs for them (and all the entries marked 3) showed that the constructions can be carried out assuming either $\mathrm{I} \Sigma_{2}^{0}$ or the comprehension principle $\Pi_{1}^{0} \mathrm{G}$ from [23] which essentially asserts the existence of generics for (i.e., meeting each one of) any given uniformly $\Pi_{1}^{0}$ collection of dense sets. Moreover, each of them is equivalent to $\mathrm{I} \Sigma_{2}^{0}$ over $\mathrm{B} \Sigma_{2}^{0}$. This presented us with a very unusual situation. Let us consider one of these examples.

P: For every type sequence $X$ satisfying the (pairwise full) amalgamation closure conditions and having an extension function approximation, there is a (1)homogeneous model realizing exactly the types in $X$.

(1) $\mathrm{P}$ is essentially the formulation of the computable construction principle for homogeneous models that appears in the literature (see [15] and [41]). 
(2) P makes no mention of recursiveness, Turing reducibility, arithmetic, or formulas of any specified quantifier rank.

(3) The proof of $\mathrm{P}$ is nontrivial. (It requires a priority argument.)

(4) $\mathrm{P}$ is a consequence of $\mathrm{I} \Sigma_{2}^{0}$.

(5) $\mathrm{P}$ is $\Pi_{1}^{1}$-conservative over $\mathrm{I} \Sigma_{1}^{0}$ and so does not imply even $\mathrm{B} \Sigma_{2}^{0}$, let alone $\mathrm{I} \Sigma_{2}^{0}$.

(6) $\mathrm{P}+\mathrm{B} \Sigma_{2}^{0}$ is equivalent to $\mathrm{I} \Sigma_{2}^{0}$.

Thus $\mathrm{P}$ is quite unusual reverse mathematically. It is a result in the standard literature with a proof not primarily by induction. Indeed, it makes no explicit mention of induction, recursiveness, or formulas in the arithmetic hierarchy. Nonetheless, it occupies a place that is within the usual hierarchy of induction axioms but is different from all the standard ones. We then thought that perhaps we could capture the combinatorial essence of these arguments in a way that would isolate the reverse mathematical issues involved. This attempt led us to an approximation version of $\Pi_{1}^{0} \mathrm{G}$ that we call $\Pi_{1}^{0} \mathrm{GA}$. Basically, it asserts not that there is a generic for uniformly $\Pi_{1}^{0}$ classes of dense sets but rather that there is a function approximating such a generic. (Here approximation is in the sense of the Shoenfield limit lemma which provides computable functions $f(x, s)$ approximating any function $g \leqslant_{\mathrm{T}} 0^{\prime}$ such that $(\forall n)(\exists t)(\forall s \geqslant t)(\forall x \leqslant n)[f(x, s)=g(x)]$. The formal definition is given after the proof of Theorem 3.1.)

This principle, $\Pi_{1}^{0} \mathrm{GA}$, is indeed sufficient to prove all the entries marked 3 in Table 6.1 (as well as those marked 4) and has much the same quite unusual reverse mathematical status. It is not provable in $\mathrm{RCA}_{0}$. It is provable from both $\mathrm{I} \Sigma_{2}^{0}$ and $\Pi_{1}^{0} \mathrm{G}$, so it is strictly weaker than each of them, and indeed (like $\Pi_{1}^{0} \mathrm{G}$ ), is highly conservative over $\mathrm{RCA}_{0}$ and so, in particular, does not prove, for instance, $\mathrm{B} \Sigma_{2}^{0}$ or $\mathrm{WKL}_{0}$. On the other hand, like the principle $\mathrm{P}$ above, it joins $\mathrm{B} \Sigma_{2}^{0}$ up to $\mathrm{I} \Sigma_{2}^{0}$ in the sense that $\mathrm{I} \Sigma_{2}^{0}$ is strictly stronger than each of these principles and is implied by their conjunction. We do not know of any principles other than the ones considered in this paper (except for trivial ones such as $\mathrm{B} \Sigma_{2}^{0} \rightarrow \mathrm{I} \Sigma_{2}^{0}$ ) with such properties. Our investigations also led to some properties of trees and their isolated paths. One gives an equivalent of $\mathrm{B} \Sigma_{2}^{0}$ (Proposition B.1). A new one (FATT) is strictly weaker even than $\mathrm{B} \Sigma_{2}^{0}$ but nonetheless not provable in $\mathrm{RCA}_{0}$ (Theorem B.2).

In the other direction, we also move up the induction hierarchy in Appendix A. There we present natural generalizations $\Pi_{n}^{0} \mathrm{GA}$ of $\Pi_{1}^{0} \mathrm{GA}$ to all levels of the arithmetic hierarchy. Essentially they say that there are approximations (in the sense of the iterated limit lemma) to generics for all uniformly $\Pi_{n}^{0}$ sequences of dense sets. The primary reverse mathematical properties of $\Pi_{1}^{0} \mathrm{GA}$ are then replicated all the way up the hierarchy:

(1) $\mathrm{I} \Sigma_{n+1}^{0} \vdash \Pi_{n}^{0} \mathrm{GA}$ (Theorem A.1).

(2) $\Pi_{n}^{0} \mathrm{GA} \nvdash \mathrm{B} \Sigma_{2}^{0}$. (The discussion before Theorem A.1 shows that $\Pi_{n}^{0} \mathrm{GA}$ is $\Pi_{1}^{1}$ conservative (and more) over $\mathrm{RCA}_{0}$.)

(3) $\mathrm{RCA}_{0} \vdash \Pi_{n}^{0} \mathrm{G}+\mathrm{B} \Sigma_{n}^{0} \leftrightarrow \mathrm{I} \Sigma_{n}^{0}$ (Theorem A.2).

Thus our reverse mathematical investigations of one model theoretic theorem have led us to a new hierarchy of combinatorial principles that are interspersed among the standard induction hierarchy in a very interesting and unusual way.

A number of questions about the model theoretic and combinatorial principles that we have investigated, and especially their reverse mathematical strength, are still open. We collect the most natural ones in Chapter 8. Perhaps the most 
interesting from a foundational rather than simply technical point of view are the ones asking for a precise determination of the reverse mathematical strength of groups of principles. In particular Question 8.6 asks whether all the entries marked 3 in Table 6.1 are equivalent and, if so, whether they are all equivalent to $\Pi_{1}^{0} \mathrm{GA}$. If, indeed, all of these principles are equivalent, this would give some evidence for the robustness of $\Pi_{1}^{0} \mathrm{GA}$ as discussed above.

Returning to the relationship between reverse and computable model theory, we would like to point to a general theme brought out by our investigations here as well as those in [23]. The motivating issue is the phenomenon in computable model theory of what one might call partial effectivization. One begins with a classical type of model such as atomic (prime) or (strongly 1-) homogeneous and the question of how hard it is to construct such a model. Typically, the effective version assumes that the underlying theory is decidable and asks about the the possible degrees of models of the desired kind and, in particular, whether (or under what conditions) there is a decidable one.

Our investigations of these questions from the viewpoint of reverse mathematics point out that the standard computability theoretic work ignores the issue of the complexity of verifying that the model constructed is of the desired kind and just accepts the classical proof. Often, there is no difference. When, however, the definition of the kind of model is not first order but calls for the existence of morphisms, for example, then additional effectiveness considerations can be raised about the complexity of the morphism required to verify the construction. A reverse mathematical analysis automatically levels the playing field for both the construction and verification. In the reverse mathematical setting, one can show that a model constructed is prime or strongly 1-homogeneous only by showing that the morphisms required can also be constructed in the same system. It makes sense to carry over these questions to computable model theory and ask how complicated (in terms of Turing degree) it is to show that the morphisms exist.

As an illustration, we take the two aforementioned topics investigated reverse mathematically in $[\mathbf{2 3}],[\mathbf{3}]$, and this paper: the existence of prime models for every atomic theory (PMT) and the existence of strongly 1-homogeneous models for every theory.

As we have mentioned, $[\mathbf{9}]$ shows that given a decidable atomic theory $T$, every $\mathbf{d} \leqslant \mathbf{0}^{\prime}$ with $\mathbf{d}^{\prime \prime}>\mathbf{0}^{\prime \prime}$ computes a prime model $\mathcal{M}$ of $T$. However, if we look at the verification that the $\mathcal{M}$ computable from $\mathbf{d}$ is prime (rather than atomic), we are required to produce, for every $\mathcal{N} \vDash T$, an elementary embedding $f: \mathcal{N} \rightarrow \mathcal{M}$. Now even if $\mathcal{N}$ is decidable there may be no such $f$ computable even in $\mathbf{d}$. From the viewpoint of reverse mathematics $[\mathbf{2 3}]$ shows that PMT is equivalent to $\mathrm{ACA}_{0}$. Thus in terms of a closure operation one needs the jump operator and the morphisms are arithmetic in the data. However, the computable model theorist should look more carefully at the question. What is then seen is that one can always construct the required $f$ computably in $T^{\prime} \oplus \mathcal{M} \oplus \mathcal{N}$ (for any prime model $\mathcal{M}$ of $T$ and any model $\mathcal{N}$ of $T$ ). The proof of the reverse mathematical equivalence then shows that this level of complexity is necessary.

Turning to strongly 1-homogeneous models, [8] shows that given a decidable $T$ one can construct a 1 -homogeneous model $\mathcal{M}$ computably in any PA degree. To verify that the model $\mathcal{M}$ is strongly 1 -homogeneous one must construct, for every pair of tuples $\bar{a}$ and $\bar{b}$ from $M$ with the same type, an automorphism $f$ of 
$\mathcal{M}$ taking $\bar{a}$ to $\bar{b}$. Now for an arbitrary 1-homogeneous model, it takes the jump of $\mathcal{M}$ to construct such an automorphism. This can be seen from the proof that reverse mathematically this general implication is equivalent to $\mathrm{ACA}_{0}$ (Proposition 4.2). The computable model theorist can easily verify that $\mathcal{M}^{\prime}$ is also sufficient. On the other hand, as we have noted, Lange [26] and Belanger [3] have shown that the existence of strongly 1-homogeneous models for every theory is equivalent to $\mathrm{WKL}_{0}$. Belanger's proof explicitly shows that if one constructs $\mathcal{M}$ still computably in a PA degree $\mathbf{d}$ but carefully, then one can also simultaneously construct all the required automorphisms computably in $\mathbf{d}$.

Thus our reverse mathematical investigations suggest a general type of problem for computable model theory. When one proves that there is a decidable or $\mathbf{d}$ decidable model $\mathcal{M}$ with some property that requires (in its definition) the existence of more than the elementary diagram of $\mathcal{M}$, then one should ask how hard it is to compute the other objects (e.g. morphisms) required to verify that the definition holds of $\mathcal{M}$. Other natural examples for investigation include issues dealing with categoricity for theories (as is common in model theory, rather than for structures as is common in computable model theory), universality, and saturation. Some of these questions have recently been investigated reverse mathematically by Belanger in $[\mathbf{2}]$ and $[\mathbf{3}]$.

Another general connection between reverse and computable mathematics (as well as degree classes more broadly) is suggested by the various notions of bounding degrees and their analyses as discussed above. Given any principle expressed by a sentence of the form $\forall X \exists Y \Phi(X, Y)$ we can form a bounding operator $\mathcal{B}_{\Phi}: \mathcal{D} \rightarrow$ $\mathcal{P}(\mathcal{D})$ (where $\mathcal{D}$ is the set of Turing degrees) defined by $\mathcal{B}_{\Phi}(\mathbf{x})=\left\{\mathbf{y} \mid\left(\forall Z \leqslant_{\mathrm{T}}\right.\right.$ $\left.\mathbf{x})\left(\exists W \leqslant_{\mathrm{T}} \mathbf{y}\right) \Phi(Z, W)\right\}$. Examples considered in this paper include the ones generated by relativizing the atomic, homogeneous, and model bounding degrees to an arbitrary $\mathbf{x}$. Indeed, most principles studied in reverse mathematics are of this form with $\Phi$ arithmetic, or at times $\Pi_{1}^{1}$. Note that the images of these operators are always upward closed subsets of $\mathcal{D}$. More generally, we can consider almost jump operators $\mathcal{J}$ that take degrees $\mathbf{x}$ to upward closed subsets $\mathcal{J}(\mathbf{x})$ of $\mathcal{D}$ (typically contained in the cone above $\mathbf{x}$ ). Examples include ones generated from all the usual (definable) jump operators $J$, from the Turing jump to sharps, by taking the image of $\mathbf{x}$ to be the cone above $J(\mathbf{x})$. The natural partial order $\leqslant$ (and so the resulting equivalence relation $\equiv$ ) on both of these classes of operators is given by reverse inclusion, i.e., $\mathcal{J} \leqslant \mathcal{K} \Leftrightarrow(\forall \mathbf{x})[\mathcal{K}(\mathbf{x}) \subseteq \mathcal{J}(\mathbf{x})]$. Our results on the model theoretic questions studied in this paper say that the operators corresponding to AMT, HMT, and WMT are all equivalent.

The methods of comparing the strength of principles using bounding operators and computable entailment are closely connected to the computability theoretic reduction procedures between $\Pi_{2}^{1}$ principles studied in papers such as [21]. Many other results in reverse and computable mathematics can be seen as saying interesting things about the natural ordering on bounding operators in general, or about specific instances of it. One that does both not only shows the existence of a minimal operator (above the one induced by the identity map on degrees) but also identifies it with a model theoretic construction principle. The obvious degree theoretic definition of this operator is given by $\Phi(X, Y) \equiv X<_{\mathrm{T}} Y$. That it corresponds to a model theoretic assertion (AST) about the existence of an atomic model of a theory with a "nice" enumeration of its types is the content of Theorem 
6.3 of $[\mathbf{2 3}]$. Another correspondence between reverse mathematical principles and standard degree classes studied in computability theory (other than the obvious ones given by the PA degrees and the Turing jump) from [23] is a type omitting theorem (OPT) shown there to be equivalent to the existence of hyperimmune sets (relative to any given set). There are many other examples that shed light on the structure of the ordering of bounding and almost jump operators. So, too, are there many questions that can be raised in this setting and approached from both the computability theoretic and reverse mathematical viewpoints.

\subsection{An outline of the paper}

In Chapter 2, we define several notions used throughout the paper.

In Chapter 3, we establish some basic facts about AMT and related principles. In particular, we introduce the principle $\Pi_{1}^{0} \mathrm{GA}$ and prove that it has the properties mentioned above. As the versions of $\Pi_{1}^{0} \mathrm{GA}$ for higher levels of the arithmetic hierarchy do not form a part of our main narrative, we leave their discussion to Appendix A. The principle FATT mentioned above, which is also related to AMT, is discussed in Appendix B.

In Chapter 4, we compare several classically equivalent definitions of homogeneity from the reverse mathematical point of view. We also analyze the strength of theorems relating homogeneous models to atomic, prime, and saturated models. One tangential result on saturated models is left to Appendix C.

In Chapter 5, we introduce the characterizations by Goncharov and Peretyat'kin of possible type spectra of (homogeneous) models. We give several classically equivalent versions of the conditions in these results, and compare them reverse mathematically. We also analyze the strength of the easier direction of these results (that the type spectrum of a (homogeneous) model must satisfy these conditions). Finally, in Section 5.5, we introduce HMT and its variants, including ones such as WMT concerning the existence of general models (rather than homogeneous ones).

In Section 6.1, we discuss the effective versions of the above characterizations (also due to Goncharov and Peretyat'kin). In particular, we introduce the notions of extension function and extension function approximation central to these effective versions, and compare them reverse mathematically. In Section 6.2, we study the reverse mathematics of several versions of the computability theoretic results of Goncharov and Peretyat'kin, summarizing our results in Tables 6.1 and 6.2 (see also Tables 6.4 and 6.5). As mentioned above, along with versions provable in $\mathrm{RCA}_{0}$ and ones equivalent to $\mathrm{I} \Sigma_{2}^{0}$, we obtain several that exhibit behavior similar to that of $\Pi_{1}^{0} \mathrm{GA}$.

In Chapter 7, we study principles asserting the existence of extension function approximations under various versions of the conditions of Goncharov and Peretyat'kin, and use our results to compare versions of AMT and HMT reverse mathematically and computability theoretically. We show in particular that AMT, HMT, and WMT are equivalent, both over $\mathrm{RCA}_{0}$ and in the sense of uniform computability theoretic reducibility.

Finally, in Chapter 8, we gather several open questions arising from our work. 



\section{CHAPTER 2}

\section{Definitions}

A few definitions given in this chapter have already been mentioned in the introduction, but we include them here as well for ease of reference.

\subsection{Reverse mathematics}

We assume familiarity with the basics of reverse mathematics, but briefly describe some of its commonly studied axiom systems. For a complete introduction to the field, see Simpson [47]. For a shorter introduction focused on combinatorial and model theoretic principles, see Hirschfeldt [20]. We work in the language of second order arithmetic, with lower case letters representing number variables and uppercase letters representing set variables. In particular, every set we consider is assumed to be countable. We think of first order objects, such as finite strings, as encoded by natural numbers, and of second order objects, such as trees or models, as encoded by sets of natural numbers (see [47] for more details).

Let $P$ consist of axioms stating that the natural numbers form a discrete ordered commutative semiring, together with set induction:

$$
(0 \in X \wedge(\forall n)[n \in X \rightarrow n+1 \in X]) \rightarrow(\forall n) n \in X
$$

Let $\mathrm{I} \Sigma_{n}^{0}$ be the following induction principle, expressed as an axiom scheme.

$$
(\varphi(0) \wedge(\forall n)[\varphi(n) \rightarrow \varphi(n+1)]) \rightarrow(\forall n) \varphi(n)
$$

for all $\Sigma_{n}^{0}$ formulas $\varphi$.

Our base axiom system is $\mathrm{RCA}_{0}$, which consists of $P$ together with $\mathrm{I} \Sigma_{1}^{0}$ and the following set existence axiom scheme, which is just strong enough to prove the existence of computable sets.

$$
(\forall n)[\varphi(n) \leftrightarrow \psi(n)] \rightarrow(\exists X)(\forall n)[n \in X \leftrightarrow \varphi(n)]
$$

for all $\Sigma_{1}^{0}$ formulas $\varphi$ and $\Pi_{1}^{0}$ formulas $\psi$ in which $X$ does not occur free.

A useful principle provable in $\mathrm{RCA}_{0}$ is bounded $\Sigma_{1}^{0}$-comprehension (see [47]), which for our purposes we state as follows. For any finite sequence $s$ of natural numbers and any $\Sigma_{1}^{0}$ property $P$, there is a subsequence of $s$ consisting of those elements of $s$ that satisfy $P$. Of course, by taking complements, bounded $\Pi_{1}^{0}$ comprehension also holds in $\mathrm{RCA}_{0}$.

Another useful fact is that $\mathrm{I} \Sigma_{2}^{0}$ is equivalent over $\mathrm{RCA}_{0}$ to the finite $\Pi_{1}^{0}$ recursion principle (see [22]), which states that if $\varphi$ is a $\Pi_{1}^{0}$ formula defining a total function, then for each $z$ and $n$, there is a sequence $x_{0}, \ldots, x_{n}$ such that $x_{0}=z$ and $\varphi\left(x_{i}, x_{i+1}\right)$ holds for all $i<n$.

Another collection of axioms schemes, related to $\mathrm{I} \Sigma_{n}^{0}$, is that of restricted bounding principles. The bounding principle $\mathrm{B} \Sigma_{n}^{0}$ is given by the axiom scheme

$$
(\forall m)[(\forall i<m)(\exists u) \varphi(i, u) \rightarrow(\exists v)(\forall i<m)(\exists u<v) \varphi(i, u)]
$$


for all $\Sigma_{n}^{0}$ formulas $\varphi$ in which $v$ does not occur free. Over $\mathrm{RCA}_{0}, \mathrm{I} \Sigma_{n}^{0}$ implies $\mathrm{B} \Sigma_{n}^{0}$, and $\mathrm{B} \Sigma_{n}^{0}$ implies $\mathrm{I} \Sigma_{n-1}^{0}$; these implications are strict (Paris and Kirby [40]; see also Hájek and Pudlák [17]).

The principle $\mathrm{B} \Pi_{n}^{0}$ is defined in the analogous way. We use the fact that $\mathrm{B} \Sigma_{2}^{0}$ and $\mathrm{B}_{1}^{0}$ are equivalent over $\mathrm{RCA}_{0}$ (see $[\mathbf{1 7}]$ ). Another useful principle equivalent to $\mathrm{B} \Sigma_{2}^{0}$ over $\mathrm{RCA}_{0}$ is the finite axiom of choice for $\Pi_{1}^{0}$ properties (see $[\mathbf{1 7}]$ ): for any sequence $n_{0}, \ldots, n_{k}$ and any $\Pi_{1}^{0}$ property $P$, if for each $i \leqslant k$ there is an $m$ with $P\left(n_{i}, m\right)$, then there is a sequence $m_{0}, \ldots, m_{k}$ such that $P\left(n_{i}, m_{i}\right)$ for each $i \leqslant k$.

$\mathrm{WKL}_{0}$ is the axiom system that includes $\mathrm{RCA}_{0}$ and the principle known as Weak König's Lemma, which states that every infinite subtree of $2^{<\mathbb{N}}$ has an infinite path. (Here a tree is a set of strings closed under initial segments.) This system is strongly related to degrees of completions of Peano arithmetic.

The last major system we consider here is $\mathrm{ACA}_{0}$, which consists of $\mathrm{RCA}_{0}$ together with arithmetic comprehension:

$$
(\exists X)(\forall n)[n \in X \leftrightarrow \varphi(n)]
$$

for every arithmetic formula $\varphi$ in which $X$ does not occur free, where a formula is arithmetic if it has no set quantifiers. Computability theoretically, $\mathrm{ACA}_{0}$ corresponds to the existence of the Turing jump.

The system $\mathrm{ACA}_{0}$ is strictly stronger than $\mathrm{WKL}_{0}$, which is in turn strictly stronger than $\mathrm{RCA}_{0}$ (see Simpson $[\mathbf{4 7}]$ ). We use the fact that $\mathrm{ACA}_{0}$ is equivalent over $\mathrm{RCA}_{0}$ to the statement that for every function $f: \mathbb{N} \rightarrow \mathbb{N}$, the range of $f$ exists, i.e., there is a set $R$ such that $n \in R$ if and only if $(\exists m) f(m)=n$ (see [47]).

For a system $\mathcal{S}$, a principle $R$, and a collection of sentences of second-order arithmetic $\Gamma$, we say that $R$ is conservative for $\Gamma$, or $\Gamma$-conservative, over $\mathcal{S}$ if every sentence in $\Gamma$ provable in $\mathcal{S}+R$ is provable in $\mathcal{S}$. For example, a classic result of Harrington (see $[\mathbf{4 7}]$ ) is that Weak König's Lemma is $\Pi_{1}^{1}$-conservative over $\mathrm{RCA}_{0}$.

A model in the language of second order arithmetic consists of a first order part $M$, which is a structure in the usual language of first order arithmetic, a subset $\mathcal{S}$ of the power set of the domain of $M$, and the membership relation $\in$ between elements of $M$ and elements of $\mathcal{S}$. If $M$ is the standard natural numbers, we say that this model is an $\omega$-model. We denote the natural numbers by $\mathbb{N}$. Of course, when interpreting a statement in a model $(M, \mathcal{S})$ as above, $\mathbb{N}$ is interpreted as $M$. An object is finite if it is coded by a natural number. For example, when we refer to a finite sequence $n_{0}, \ldots, n_{k}$ of natural numbers, we mean that $\left\langle n_{0}, \ldots, n_{k}\right\rangle \in \mathbb{N}$, where $\langle\cdot, \ldots, \cdot\rangle$ is any standard encoding of tuples of natural numbers as single natural numbers. A set is infinite if it is unbounded. Thus, when we say that there are infinitely many natural numbers $x$ such that some property $P(x)$ holds, we mean that for each $n \in \mathbb{N}$ there is an $x>n$ such that $P(x)$ holds. Note that, for a model $(M, \mathcal{S})$ in the language of second order arithmetic, there may be $A \subset M$ that are neither finite nor infinite in the above sense, though if $(M, \mathcal{S}) \vDash \mathrm{RCA}_{0}$, then such an $A$ cannot be in $\mathcal{S}$.

\subsection{Model theoretic notions}

In this section, we introduce some notation and discuss a few concepts and results of basic model theory. See [4] or [30] for an introduction to model theory. For an overview of computable model theory, see $[\mathbf{1}]$ or $[\mathbf{1 8}]$. We assume that all theories are consistent and complete, and that all languages include equality 
(although our results for the most part do not depend on the latter assumption; see Remark 5.2 for the one exception, which is easily handled). As noted above, we think of second order model theoretic objects such as theories and models as encoded by sets of natural numbers. In particular, we assume that all languages and models are countable. We use calligraphic letters such as $\mathcal{A}$ for models, and use the corresponding roman letters such as $A$ for their domains. Whenever we mention a tuple of variables, we assume the variables in the tuple are distinct. (When we mention a tuple of elements of a model, on the other hand, we do not make this assumption.)

Definition 2.1. Let $T$ be a theory and $\mathcal{A}$ a model of $T$.

(i) A formula $\theta(\bar{x})$ is consistent with $T$ if $T \cup\{(\exists \bar{x}) \theta(\bar{x})\}$ is consistent, or equivalently, because of the completeness of $T$, if $(\exists \bar{x}) \theta(\bar{x}) \in T$.

(ii) An $n$-type $p(\bar{x})$ of $T$ in the $n$-tuple of variables $\bar{x}$ is a maximal set of formulas in variables taken from $\bar{x}$ consistent with $T$.

(iii) For a $k$-subtuple $\bar{y}$ of an $n$-tuple $\bar{x}$, and an $n$-type $p(\bar{x})$, let $p \uparrow \bar{y}$ be the $k$-type consisting of all the formulas in $p$ whose variables are all in $\bar{y}$. We call such a type a subtype of $p$.

(iv) Let $S_{n}(T)$ be the set of all $n$-types of $T$ (in all $n$-tuples of variables), and let $S(T)=\bigcup_{n \in \mathbb{N}} S_{n}(T)$.

(v) We say $p \in S_{n}(T)$ is a principal type if there exists a formula $\psi \in p$ such that $T \vdash \psi \rightarrow \theta$ for all $\theta \in p$. We call such a $\psi$ a generator of $p$ and say $\psi$ generates or isolates $p$.

(vi) A formula $\varphi(\bar{x})$ is an atom of $T$ if for every formula $\psi(\bar{x})$, either $T \vdash \varphi \rightarrow \psi$ or $T \vdash \varphi \rightarrow \neg \psi$, but not both.

(vii) The theory $T$ is atomic if for every formula $\psi$ consistent with $T$, there is an atom $\varphi$ of $T$ such that $T \vdash \varphi \rightarrow \psi$. (In other words, $T$ is atomic if every formula consistent with $T$ is an element of some principal type of $T$.)

(viii) An $n$-tuple $\bar{a} \in A$ realizes an $n$-type $p(\bar{x}) \in S_{n}(T)$ if $\mathcal{A} \vDash \theta(\bar{a})$ for all $\theta(\bar{x}) \in p(\bar{x})$. In this case we also say that $\mathcal{A}$ realizes $p$. The type of $\bar{a}$ in the variables $\bar{x}$ (where $|\bar{x}|=|\bar{a}|$ ) is the type $p(\bar{x})$ that $\bar{a}$ realizes.

(ix) The type spectrum of $\mathcal{A}$ is

$$
\mathbb{T}(\mathcal{A})=\{p \in S(T): \mathcal{A} \text { realizes } p\}
$$

(x) The model $\mathcal{A}$ is atomic if every tuple of elements of $A$ satisfies an atom of $T$. (In other words, $\mathcal{A}$ is atomic if $\mathbb{T}(\mathcal{A})$ consists of principal types.)

(xi) The model $\mathcal{A}$ is prime if it is elementarily embeddable in each model of $T$.

(xii) The model $\mathcal{A}$ is saturated if for all $\bar{a} \subseteq A$ and $p \in S_{n}(\operatorname{Th}(\mathcal{A}, \bar{a}))$, the type $p$ is realized in $\mathcal{A}$, where $(\mathcal{A}, \bar{a})$ is the expanded structure obtained by adding to $\mathcal{A}$ constants for the elements of $\bar{a}$, and $\operatorname{Th}(\mathcal{A}, \bar{a})$ is the theory of this structure.

(xiii) A partial type of $T$ in the variables $\bar{x}$ is a (not necessarily maximal) set of formulas in variables taken from $\bar{x}$ consistent with $T$. The model $\mathcal{A}$ is strongly saturated if for all $\bar{a} \subseteq A$, each partial type of $\operatorname{Th}(\mathcal{A}, \bar{a})$ is realized in $\mathcal{A}$. (This notion is of course classically equivalent to being saturated, but this equivalence is not provable in $\mathrm{RCA}_{0}$, and is in fact equivalent to $\mathrm{WKL}_{0}$; see Appendix C.) 
Since we are interested in statements about the existence of homogeneous models realizing particular sets of types, we need a way to encode lists (i.e., enumerations) of types.

Definition 2.2. Let $T$ be a theory and let $\left\{\theta_{i}\right\}_{i \in \mathbb{N}}$ be an effective listing of the formulas in the language of $T$. We identify $p \in S(T)$ with the function $f_{p} \in 2^{\mathbb{N}}$ such that $f_{p}(i)=1$ if $\theta_{i} \in p$ and $f_{p}(i)=0$ otherwise. Let $p\left\lceil s=p \cap\left\{\theta_{i}\right\}_{i<s}\right.$. We identify $p \uparrow s$ with the function $f_{p} \uparrow s$.

Let $X=\left\{p_{i}\right\}_{i \in \mathbb{N}} \subset S(T)$. We encode $X$ as a function $f(i, j)$ such that for each $i$, the first value $f(i, 0)$ encodes the set of free variables of $p_{i}$, and $f(i, k+1)=f_{p_{i}}(k)$.

Note that, in both the computability theoretic and the reverse mathematical settings, we have to deal with enumerations of potential type spectra, because even if $\left\{p_{i}\right\}_{i \in \mathbb{N}}$ and $\left\{q_{i}\right\}_{i \in \mathbb{N}}$ are equal as sets of types, there may be no effective way to go from an index $i$ to an index $j$ such that $q_{j}=p_{i}$. We say that a list of types $\left\{p_{i}\right\}_{i \in \mathbb{N}}$ is computable if the function $f$ in Definition 2.2 is computable.

In computable model theory, a model $\mathcal{A}$ can be associated with its atomic diagram or with its elementary diagram. Let $\mathcal{L}_{A}$ be the expansion of the language of $\mathcal{A}$ obtained by adding a constant symbol for each element of $\mathcal{A}$. The elementary diagram $D^{e}(\mathcal{A})$ of $\mathcal{A}$ is the set of all sentences of $\mathcal{L}_{A}$ that are true in $\mathcal{A}$ (under the obvious interpretation of the new constant symbols). The atomic diagram $D^{a}(\mathcal{A})$ of $\mathcal{A}$ is the set of all atomic sentences of $\mathcal{L}_{A}$ that are true in $\mathcal{A}$. Alternatively, we can associate these diagrams with satisfaction predicates. A predicate $S$ is an elementary (atomic) satisfaction predicate for $\mathcal{A}$ if for any (atomic) formula $\theta(\bar{c})$ with constants from $\mathcal{A}$, the predicate $S$ holds of $\theta(\bar{c})$ if and only if $\theta(\bar{c})$ is true in $\mathcal{A}$. In computable model theory, we measure the complexity of a model by the degree theoretic complexity of these diagrams. We say that $\mathcal{A}$ is computable if $D^{a}(\mathcal{A})$ is computable and that $\mathcal{A}$ is decidable if $D^{e}(\mathcal{A})$ is computable, and we similarly define the notions of $\mathbf{d}$-computable and $\mathbf{d}$-decidable for any degree $\mathbf{d}$. (Note that for a theory $T$, saying that $T$ is decidable is just another way of saying that $T$ is computable.)

When investigating the strength of theorems of model theory such as AMT and HMT, decidable models and their relativizations are the more relevant notions. Accordingly, in reverse mathematics, a model $\mathcal{A}$ includes a function interpreting the terms of the language in the model, as well as the full satisfaction predicate for $\mathcal{A}$. For a formal definition of a model in this setting, see Simpson $[4 \mathbf{4}$, Definition II.8.3].

In several places below, we specify a theory by giving a set $A$ of axioms that generates it. In each case, the language of $A$ does not include any function symbols, so all terms are either variables or constant symbols. To show that such a set $A$ does in fact specify a theory $T$, we use quantifier elimination. Recall that to show that $A$ admits effective quantifier elimination, it is enough to give a procedure to find a quantifier-free formula $\psi(\bar{x})$ that is $A$-equivalent to a given existential formula $(\exists y) \varphi(\bar{x} y)$, where $\varphi(\bar{x} y)$ is a conjunction of literals, i.e., atomic formulas and negations of atomic formulas. (We always assume that we have propositional constants $\mathrm{T}$ and $\mathrm{F}$ for true and false, respectively.) It then follows that the deductive closure $T$ of $A$ is $\Delta_{1}^{0}$-definable from $A$ and complete, assuming that the set of quantifier-free sentences consistent with $A$ is itself $\Delta_{1}^{0}$ definable from $A$. (It is easy to see that this assumption holds if the set of literal sentences (i.e., literals with no free variables) consistent with $A$ is $\Delta_{1}^{0}$, and hence that it always holds when the 
language of $A$ does not include constant symbols, as then the only literal sentences are $\mathrm{T}$ and $\mathrm{F}$.) It also follows that every type of $T$ is determined by the literals it contains, so we can identify the types of $T$ with atomic types, i.e., sets of literals $p(\bar{x})$ such that for each atomic formula $\psi$ with variables among the elements of $\bar{x}$, either $\psi \in p(\bar{x})$ or $\neg \psi \in p(\bar{x})$.

REMARK 2.3. Let $\mathcal{L}$ be the language of $T$, and let $\mathcal{L}^{\prime}$ be any finite sublanguage of $\mathcal{L}$ that includes all symbols occurring in $\varphi$. Say that a formula $\psi(\bar{u})$ is an $\mathcal{L}^{\prime}$ atomic diagram if it is a conjunction of literals in $\mathcal{L}^{\prime}$; for every atomic $\theta$ in $\mathcal{L}^{\prime}$ with variables among those in $\bar{u}$, exactly one of $\theta$ and $\neg \theta$ occurs in $\psi$; and $\psi$ is consistent with the usual rules for equality. It is easy to see that there is a procedure that, given any conjunction $\delta$ of literals in $\mathcal{L}^{\prime}$, finds a disjunction of $\mathcal{L}^{\prime}$-atomic diagrams that is equivalent to $\delta$ (or returns $\mathrm{F}$ if $\delta$ contains both $\theta$ and $\neg \theta$ for some $\theta$, or is not consistent with the rules for equality). Since existential quantifiers distribute over disjunctions, in performing an effective quantifier elimination procedure as above, we may assume that $\varphi$ is itself an $\mathcal{L}^{\prime}$-atomic diagram.

If $u=y$ occurs in $\varphi$, where $u \in \bar{x}$ or $u$ is a constant symbol, then we may replace all occurrences of $y$ in $\varphi$ by $u$ and drop the $(\exists y)$ quantifier to obtain a quantifier-free formula equivalent to $(\exists y) \varphi(\bar{x} y)$. Similarly, if $u=v$ occurs in $\varphi$, where $u \in \bar{x}$ or $u$ is a constant symbol, and $v \in \bar{x}$ is distinct from $u$, then we may replace all occurrences of $v$ in $\varphi$ by $u$ and move $u=v$ outside the existential quantifier. If we now have both $\theta$ and $\neg \theta$ occurring in $\varphi$, then $\vdash(\exists y) \varphi(\bar{x} y) \leftrightarrow \mathrm{F}$. Thus we may assume that $\varphi$ is an $\mathcal{L}^{\prime}$-atomic diagram that includes $\neg u=v$ for all $u$ such that $u \in \bar{x}$ or $u$ is a constant symbol, and all $v \in \bar{x} y$ distinct from $u$.

Suppose there is a binary relation symbol $E$ in $\mathcal{L}^{\prime}$ such that $A$ asserts that $E$ is an equivalence relation. Then there clearly is a procedure that, given a $\mathcal{L}^{\prime}$ atomic diagram $\psi$, decides whether $\psi$ is consistent with the assertion that $E$ is an equivalence relation (that is, whether $\psi$ includes $E(u, v)$ whenever it includes $u=v$, includes $E(u, v)$ whenever it includes $E(v, u)$, and includes $E(u, w)$ whenever it contains $E(u, v)$ and $E(v, w)$, where in each case, each of $u, v$, and $w$ is a variable or a constant symbol). If this is not the case, then $A \vdash(\exists y) \psi(\bar{x} y) \leftrightarrow \mathrm{F}$, so we may assume that our $\mathcal{L}^{\prime}$-atomic diagram $\varphi$ is consistent with the assertion that $E$ is an equivalence relation for any such $E$.

\subsection{Other notions}

We define a few basic notions about trees that appear in our proofs below. We discuss the connection between trees and theories in Chapter 3; for more on this topic, see Lange and Soare [29].

Definition 2.4. (i) A (binary) tree $\mathcal{T}$ is a subset of $2^{<\mathbb{N}}$ closed under initial segments, i.e., if $\sigma \in \mathcal{T}$ and $\tau \prec \sigma$ then $\tau \in \mathcal{T}$. (As this is the only kind of tree considered below, we omit the word "binary".) The set of (infinite) paths of $\mathcal{T}$ is

$$
[\mathcal{T}]=\left\{f \in 2^{\mathbb{N}}:(\forall n) f\lceil n \in \mathcal{T}\} .\right.
$$

(ii) A tree $\mathcal{T}$ is extendible if for every $\sigma \in \mathcal{T}$, at least one of $\sigma 0$ and $\sigma 1$ is in $\mathcal{T}$. (In other words, $\mathcal{T}$ is extendible if every $\sigma \in \mathcal{T}$ can be extended to an infinite path of $\mathcal{T}$.)

(iii) Suppose $\mathcal{T}$ is extendible. A node $\tau \in \mathcal{T}$ is an atom of $\mathcal{T}$ if for each $n \geqslant|\tau|$, there is exactly one $\sigma \in \mathcal{T}$ of length $n$ extending $\tau$. In other words, $\tau \in \mathcal{T}$ 
is an atom of $\mathcal{T}$ if there is only one $f \in[\mathcal{T}]$ extending $\tau$. We say that this $f$ is isolated, that $\tau$ is a generator of $f$, and that $\tau$ isolates or generates $f$.

(iv) An extendible tree $\mathcal{T}$ is atomic if for every $\sigma \in \mathcal{T}$ there is an atom $\tau \in \mathcal{T}$ extending $\sigma$. It is strongly atomic if for every finite collection $\sigma_{0}, \ldots, \sigma_{n} \in \mathcal{T}$ there is a finite collection $\tau_{0}, \ldots, \tau_{n}$ of atoms of $\mathcal{T}$ such that each $\tau_{i}$ extends $\sigma_{i}$. (Of course, every atomic tree is strongly atomic, but this fact cannot be proved in $\mathrm{RCA}_{0}$; see Appendix B.) 


\section{CHAPTER 3}

\section{The Atomic Model Theorem and Related Principles}

In this chapter, we discuss a few principles that play a significant role below. As mentioned in the introduction, Hirschfeldt, Shore, and Slaman [23] studied the reverse mathematical strength of the following basic model theoretic fact.

AMT (Atomic Model Theorem): Every atomic theory has an atomic model. They showed that AMT is a fairly weak principle, but is nevertheless not provable in $\mathrm{WKL}_{0}$. (See $[\mathbf{2 3}]$ for details.)

AMT is also closely related to the following genericity principle. (When we say that $D$ is a $\Pi_{1}^{0}$ set of strings, we mean that $D$ is a $\Pi_{1}^{0}$ predicate on $2^{<\mathbb{N}}$, and write $\sigma \in D$ to mean that $D$ holds of $\sigma$. This notation does not imply that $D$ exists as a set.)

$\Pi_{1}^{0} \mathbf{G}$ : For any uniformly $\Pi_{1}^{0}$ collection of sets $D_{i}$, each of which is dense in $2^{<\mathbb{N}}$, there is a $G$ such that $(\forall i)(\exists m)\left[G\left\lceil m \in D_{i}\right]\right.$.

Conidis $[\mathbf{6}]$ showed that $\mathrm{AMT}$ and $\Pi_{1}^{0} \mathrm{G}$ are computability theoretic equivalent. In the context of reverse mathematics, his result shows that $\mathrm{RCA}_{0}+\mathrm{AMT}$ and $\mathrm{RCA}_{0}+$ $\Pi_{1}^{0} \mathrm{G}$ have the same $\omega$-models. Subsequently, Hirschfeldt, Shore, and Slaman $[\mathbf{2 3}]$ showed that $\Pi_{1}^{0} \mathrm{G}$ implies AMT over $\mathrm{RCA}_{0}$, while AMT implies $\Pi_{1}^{0} \mathrm{G}$ over $\mathrm{RCA}_{0}+$ $\mathrm{I} \Sigma_{2}^{0}$, but not over $\mathrm{RCA}_{0}$. They also established the following conservativity results.

Theorem 3.1 (Hirschfeldt, Shore, and Slaman [23]).

(1) AMT and $\Pi_{1}^{0} \mathrm{G}$ are restricted $\Pi_{2}^{1}$-conservative over $\mathrm{RCA}_{0}$, i.e., conservative over $\mathrm{RCA}_{0}$ for sentences of the form

$$
(\forall A)[\Theta(A) \rightarrow(\exists B) \Phi(A, B)]
$$

where $\Theta$ is arithmetic and $\Phi$ is $\Sigma_{3}^{0}$. In particular, AMT and $\Pi_{1}^{0} \mathrm{G}$ are $\Pi_{1}^{1}$ conservative over $\mathrm{RCA}_{0}$.

(2) AMT is $\Pi_{1}^{1}$-conservative over $\mathrm{RCA}_{0}+\mathrm{B} \Sigma_{2}^{0}$.

On the other hand, they showed that $\Pi_{1}^{0} \mathrm{G}$ implies $\mathrm{I} \Sigma_{2}^{0}$ over $\mathrm{RCA}+\mathrm{B} \Sigma_{2}^{0}$. In Section 6.2 , we discuss several model theoretic principles provable from $\mathrm{RCA}_{0}+\Pi_{1}^{0} \mathrm{G}$ that likewise imply $\mathrm{I} \Sigma_{2}^{0}$ over $\mathrm{RCA} \mathrm{A}_{0}+\mathrm{B} \Sigma_{2}^{0}$. Unlike $\Pi_{1}^{0} \mathrm{G}$, however, these principles are true in every $\omega$-model of $\mathrm{RCA}_{0}$, and indeed are provable in $\mathrm{RCA}_{0}+\mathrm{I} \Sigma_{2}^{0}$. It thus becomes interesting to find a combinatorial principle, along the lines of $\Pi_{1}^{0} \mathrm{G}$, living in that same part of the reverse mathematical universe. We obtain such a principle by replacing the statement that $\Pi_{1}^{0}$-generics exist by the statement that they can be approximated:

$\Pi_{1}^{0}$ GA: For any uniformly $\Pi_{1}^{0}$ collection of sets of strings $D_{0}, D_{1}, \ldots$, each of which is dense in $2^{<\mathbb{N}}$, there is a sequence $g_{0}, g_{1}, \ldots \in 2^{\mathbb{N}}$ such that

$$
(\forall i)(\exists m)(\exists t)(\forall u>t)\left[g _ { u } \left\lceilm=g_{t}\left\lceil m \in D_{i}\right] .\right.\right.
$$


Note that this principle is equivalent to the version where we add the requirement that $(\forall n)(\exists t)(\forall u>t)\left[g_{u} \uparrow n=g_{t} \uparrow n\right]$, since we can add to the list $D_{0}, D_{1}, \ldots$ all sets of the form $\{\sigma:|\sigma| \geqslant n\}$, and if $m \geqslant n$, then $g_{u} \uparrow m=g_{t} \uparrow m$ implies $g_{u}\left\lceil n=g_{t}\lceil n\right.$.

Of course, $\Pi_{1}^{0} \mathrm{G}$ implies $\Pi_{1}^{0} \mathrm{GA}$, so conservativity results for the former remain true of the latter. In particular, $\Pi_{1}^{0} \mathrm{GA}$ does not imply $\mathrm{B} \Sigma_{2}^{0}$ over $\mathrm{RCA}_{0}$. The following two theorems show that $\Pi_{1}^{0} \mathrm{GA}$ is in fact equivalent to $\mathrm{I} \Sigma_{2}^{0}$ over $\mathrm{B} \Sigma_{2}^{0}$.

THEOREM 3.2. $\mathrm{RCA}_{0}+\mathrm{I}_{2}^{0} \vdash \Pi_{1}^{0} \mathrm{GA}$.

ProOF. We argue in $\mathrm{RCA}_{0}+\mathrm{I} \Sigma_{2}^{0}$. Let $D_{0}, D_{1}, \ldots$ be a uniformly $\Pi_{1}^{0}$ collection of sets of strings, each of which is dense in $2^{<\mathbb{N}}$. Let $D_{i}[s]$ be the stage $s$ approximation to $D_{i}$. For each $s$, define $\sigma_{s, i}$ for $i \leqslant s$ by recursion as follows. Let $\sigma_{s, 0}$ be the length-lexicographically least string in $D_{0}[s]$. Given $\sigma_{s, i-1}$, let $\sigma_{s, i}$ be the length-lexicographically least extension of $\sigma_{s, i-1}$ in $D_{i}[s]$. Each $D_{i}[s]$ contains $D_{i}$, and hence is dense, so the $\sigma_{s, i}$ are all well-defined. Let $g_{s}=\sigma_{s, s} 0^{\mathbb{N}}$.

Consider the statement

$$
(\exists t \geqslant i)(\forall u>t)\left[\sigma_{u, i}=\sigma_{t, i}\right]
$$

This statement clearly holds of $i=0$, since there are a string $\sigma$ and a $t$ such that $\sigma \in D_{0}$ and $\tau \notin D_{0}[t]$ for all $\tau$ that are length-lexicographically less than $\sigma$. By a similar argument, if the statement holds of $i$, then it holds of $i+1$. Thus, by $\mathrm{I} \Sigma_{2}^{0}$, the statement holds of all $i$. Fix $i$ and let $t$ be as in the above statement. Then $\sigma_{t, i} \in D_{i}$, since $\sigma_{t, i}=\sigma_{u, i} \in D_{i}[u]$ for all $u \geqslant t$. Furthermore, $g_{u}$ extends $\sigma_{t, i}$ for all $u \geqslant t$, so $(\exists m)(\exists t)(\forall u>t)\left[g_{u}\left\lceil m=g_{t}\left\lceil m \in D_{i}\right]\right.\right.$.

The following proof is similar to that of the corresponding result for $\Pi_{1}^{0} \mathrm{G}$ in Hirschfeldt, Shore, and Slaman [23]. $\mathrm{I} \Sigma_{2}^{0}$

Theorem 3.3. $\mathrm{RCA}_{0}+\mathrm{B} \Sigma_{2}^{0}+\Pi_{1}^{0} \mathrm{GA} \vdash \mathrm{I} \Sigma_{2}^{0}$, and hence $\mathrm{RCA}_{0}+\mathrm{B} \Sigma_{2}^{0} \vdash \Pi_{1}^{0} \mathrm{GA} \leftrightarrow$

Proof. Fix a model of $\mathrm{RCA}_{0}+\mathrm{B} \Sigma_{2}^{0}+\Pi_{1}^{0} \mathrm{GA}$ with first order part $M$ and second order part $\mathcal{S}$. Suppose for the sake of a contradiction that $I \Sigma_{2}^{0}$ fails in this model. Then there is a $\Sigma_{2}^{0}$ cut $\mathcal{I}$ in $M$; i.e., $x \in \mathcal{I} \Leftrightarrow(\exists y)(\forall z) \varphi(x, y, z)$ for some $\Delta_{0}^{0}$ formula $\varphi$ and $\mathcal{I}$ is a proper initial segment of $M$ closed under successor. Let $D_{i}$ be the set of all $\sigma \in 2^{<\mathbb{N}}$ such that, thinking of $\sigma$ as a finite set, the last two elements of $\sigma$, in order, are $w$ and $\langle i, x, y\rangle$, and

$$
\left(\exists y^{\prime}<y\right)(\forall z)\left[\varphi\left(x, y^{\prime}, z\right) \wedge\left(\forall y^{\prime} \leqslant w\right)\left(\exists z^{\prime}<y\right) \neg \varphi\left(x, y^{\prime}, z^{\prime}\right)\right] .
$$

Clearly the $D_{i}$ are uniformly $\Pi_{1}^{0}$. As $\mathcal{I}$ is a cut and so not a member of $\mathcal{S}$, there is no bound on the least witnesses $y$ such that $(\forall z) \varphi(x, y, z)$ for $x \in \mathcal{I}$, while by $\mathrm{I} \Sigma_{1}^{0}$ every $x \in \mathcal{I}$ has such a least witness $y$, and by $\mathrm{B} \Sigma_{2}^{0}$ there is a bound on the $z^{\prime}$ needed to show that $y$ is in fact the least witness for $x$. Thus each $D_{i}$ is dense, for if we consider any $\tau$ with last element $w$ there is an $x \in \mathcal{I}$ whose least witness $y$ is larger than $w$ and a $z>y$ such that counterexamples for all $y^{\prime} \leqslant w$ can be found below $z$. So if we let $\sigma$ extend $\tau$ by adding on as its only new element $\langle i, x, z\rangle$ we have our extension of $\tau$ in $D_{i}$ as required.

Let $g_{0}, g_{1}, \ldots$ be as in $\Pi_{1}^{0} \mathrm{GA}$ and let $a$ be any number above all those in $\mathcal{I}$. Let $E_{i}=\left\{\langle m, t\rangle:(\forall u \geqslant t)\left[g_{u} \uparrow m=g_{t} \uparrow m \in D_{i}[u]\right]\right\}$. Note that the $E_{i}$ are uniformly $\Pi_{1}^{0}$. We have $(\forall i<a)(\exists\langle m, t\rangle)\langle m, t\rangle \in E_{i}$, so by the finite axiom of choice for $\Pi_{1}^{0}$ properties, there is a function $f$ with domain $a$ that is $M$-finite (i.e., coded in $M$ 
by a number) and such that for each $i<a$ there is an $\langle m, t\rangle \in E_{i}$ with the last element of $g_{t} \uparrow m$ of the form $\langle i, f(i), y\rangle$. Since $\langle m, t\rangle \in E_{i} \Rightarrow g_{t} \uparrow m \in D_{i}$, every $f(i)$ is in $\mathcal{I}$.

We claim that $f$ is one-to-one. Since $\mathcal{I}$ is a proper subset of $a$, this fact gives us a contradiction, as we can show in $\mathrm{RCA}_{0}$ that no such function exists. So assume for a contradiction that $f(i)=f(j)=x$ for $i \neq j$. Then there are $\langle m, t\rangle \in E_{i}$ and $\left\langle m^{\prime}, t^{\prime}\right\rangle \in E_{j}$, and there are $v, w, y_{i}, y_{j}$, such that the next to last and last elements of $g_{t} \uparrow m$ are $v$ and $\left\langle i, x, y_{i}\right\rangle$, and the next to last and last elements of $g_{t^{\prime}} \uparrow m^{\prime}$ are $w$ and $\left\langle j, x, y_{j}\right\rangle$. Let $u=\max \left(t, t^{\prime}\right)$. By the definitions of $E_{i}$ and $E_{j}$, we have $g_{u} \uparrow m=g_{t} \uparrow m$ and $g_{u} \uparrow m^{\prime}=g_{t^{\prime}} \uparrow m^{\prime}$. Thus we cannot have $m=m^{\prime}$, since $g_{t} \uparrow m$ and $g_{t^{\prime}} \uparrow m^{\prime}$ have different last elements. So, without loss of generality, we may assume that $m<m^{\prime}$ and so $v<\left\langle i, x, y_{i}\right\rangle \leqslant w<\left\langle j, x, y_{j}\right\rangle$. We now have a contradiction, as the definitions of $D_{i}$ and $D_{j}$ then say that $\left(\exists y^{\prime}<y_{i}\right)(\forall z) \varphi\left(x, y^{\prime}, z\right)$ and $\left(\forall y^{\prime} \leqslant w\right)\left(\exists z^{\prime}<y\right) \neg \varphi\left(x, y^{\prime}, z^{\prime}\right)$, respectively, but $y_{i} \leqslant w$.

Corollary 3.4. AMT does not imply $\Pi_{1}^{0} \mathrm{GA}$ over $\mathrm{RCA}_{0}$.

One can think of $\Pi_{1}^{0} \mathrm{GA}$ as an attempt to capture the first order difference between AMT and $\Pi_{1}^{0} \mathrm{G}$, but we do not know whether AMT $+\Pi_{1}^{0} \mathrm{GA}$ implies $\Pi_{1}^{0} \mathrm{G}$ over $\mathrm{RCA}_{0}$.

There are principles analogous to $\Pi_{1}^{0} \mathrm{GA}$ for higher levels of the arithmetic hierarchy, and results corresponding to the above two theorems hold for these principles. Since these results, while of independent interest, are not needed in the rest of this paper, we leave them to Appendix A.

Let $T$ be an atomic theory. From an atomic model of $T$, we can easily obtain a listing of the principal types of $T$. By applying Shore blocking to a priority argument of Goncharov and Nurtazin [16] and Harrington [19], Hirschfeldt, Shore, and Slaman [23] showed that it is also provable in $\mathrm{RCA}_{0}$ that if there is a listing of the principal types of $T$, then $T$ has an atomic model. Thus AMT can be restated as "If $T$ is an atomic theory, then there is a listing of the principal types of $T$."

There is a fairly straightforward translation between theories and extendible trees. In one direction, given a theory $T$, we can consider the tree $\mathcal{T}$ of types of $T$. More precisely, we define $\mathcal{T}$ as follows. For each $n$, let

$$
\varphi_{0}^{n}\left(x_{0}, \ldots, x_{n-1}\right), \varphi_{1}^{n}\left(x_{0}, \ldots, x_{n-1}\right), \ldots
$$

list the formulas with free variables among $x_{0}, \ldots, x_{n-1}$ in the language of $T$. For $\sigma \in 2^{<\mathbb{N}}$, let

$$
\varphi_{\sigma}^{n} \equiv \bigwedge_{i: \sigma(i)=1} \varphi_{i}^{n} \wedge \bigwedge_{i: \sigma(i)=0} \neg \varphi_{i}^{n}
$$

Let $\mathcal{T}$ consist of all strings of the form $0^{n}$ and all strings of the form $0^{n} 1 \sigma$ such that $\varphi_{\sigma}^{n}$ is consistent with $T$. It is easy to show in $\mathrm{RCA}_{0}$ that $\mathcal{T}$ is an extendible tree, that if $T$ is atomic then $\mathcal{T}$ is strongly atomic, and that if there is a listing of the isolated paths of $\mathcal{T}$, then there is a listing of the principal types of $T$.

In the other direction, we have the following construction.

Definition 3.5. Let $\mathcal{L}$ be the language $\left\{P_{i}\right\}_{i \in \mathbb{N}}$, where each $P_{i}$ is a unary relation symbol. For $\sigma \in 2^{<\mathbb{N}}$, let $P^{\sigma}(y)$ be the formula $\bigwedge_{n=0}^{|\sigma|-1} P_{n}^{\sigma(n)}(y)$, where $P_{n}^{1}(y)$ denotes the formula $P_{n}(y)$ and $P_{n}^{0}(y)$ denotes the formula $\neg P_{n}(y)$. For a $k$-tuple $s=\left(\sigma_{0}, \ldots, \sigma_{k-1}\right)$ of elements of $2^{<\mathbb{N}}$, let $P^{s}\left(y_{0}, \ldots y_{k-1}\right)$ be the formula $\bigwedge_{i<k} P^{\sigma_{i}}\left(y_{i}\right)$. 
Given an extendible tree $\mathcal{T} \subseteq 2^{<\mathbb{N}}$, let $T(\mathcal{T})$ be the deductive closure in $\mathcal{L}$ of the following set $R(\mathcal{T})$ of axioms.

(1) $\left(\exists^{>m} x\right) P^{\sigma}(x)$ for all $\sigma \in \mathcal{T}$ and $m \in \mathbb{N}$.

(2) $\neg(\exists x) P^{\sigma}(x)$ for all $\sigma \notin \mathcal{T}$.

We can clearly prove the existence of $R(\mathcal{T})$ in $\mathrm{RCA}_{0}$. The following lemma shows that we can do more, and establishes the correspondence between isolated paths of $\mathcal{T}$ and principal types of $T(\mathcal{T})$.

Proposition 3.6. The following hold in $\mathrm{RCA}_{0}$ for an extendible tree $\mathcal{T}$.

(1) $T(\mathcal{T})$ exists and is a theory, i.e., complete and consistent.

(2) If $\mathcal{T}$ is strongly atomic, then $T(\mathcal{T})$ is an atomic theory.

(3) From an enumeration of the principal types of $T(\mathcal{T})$, we can build an enumeration of the isolated paths of $\mathcal{T}$.

Proof. We argue in $\mathrm{RCA}_{0}$.

(1) We first show that $R(\mathcal{T})$ admits effective quantifier elimination, as discussed at the end of Section 2.2. Fix an existential formula $(\exists y) \varphi(\bar{x} y)$, where $\varphi(\bar{x} y)$ is a conjunction of literals. Let $m$ be such that no $P_{i}$ with $i>m$ occurs in $\varphi$, and let $\mathcal{L}^{\prime}$ be the language consisting of all $P_{i}$ with $i \leqslant m$. As discussed in Remark 2.3, we may assume that $\varphi$ is an $\mathcal{L}^{\prime}$-atomic diagram, and that it includes $\neg x=y$ for all $x \in \bar{x}$. Let $\sigma$ be such that $P^{\sigma}(y)$ equals the conjunction of literals in $\varphi$ of the form $P_{i}(y)$, and let $\psi$ be the conjunction of literals in $\varphi$ not involving $y$ (which is just $\mathrm{T}$ if there are no such literals). It is easy to see that if $\sigma \in \mathcal{T}$ then $R(\mathcal{T}) \vdash(\exists y) \varphi(\bar{x} y) \leftrightarrow \psi$, and otherwise, $R(\mathcal{T}) \vdash(\exists y) \varphi(\bar{x} y) \leftrightarrow$ F. Thus $R(\mathcal{T})$ admits effective quantifier elimination, and so its deductive closure exists and is complete if consistent, and hence a theory as required.

To show that $T(\mathcal{T})$ is consistent, we provide a model $\mathcal{A}$ of $T(\mathcal{T})$. Since $\mathcal{T}$ is extendible, there is a function $f: 2^{<\mathbb{N}} \times \mathbb{N} \rightarrow 2$ such that for each $\sigma \in \mathcal{T}$, if we think of $f(\sigma, \cdot)$ as an element $\alpha$ of $2^{\mathbb{N}}$, then $\alpha$ is the leftmost path of $\mathcal{T}$ extending $\sigma$. Let the domain of $\mathcal{A}$ be $\mathbb{N}$. Since $R(\mathcal{T})$ admits effective quantifier elimination and there are only unary relation symbols, we need to specify only what unary relations hold of each $n \in \mathbb{N}$. Let $\left\{\tau_{i}\right\}_{i \in \mathbb{N}}$ be an enumeration of $\mathcal{T}$. For each $i, j, k$, let $P_{k}^{f\left(\tau_{i}, k\right)}(\langle i, j\rangle)$ hold in $\mathcal{A}$. (We assume our pairing function is bijective.) Clearly $\mathcal{A}$ is a model of $T(\mathcal{T})$, and hence $T(\mathcal{T})$ is consistent.

(2) Suppose that $\mathcal{T}$ is strongly atomic, and fix a formula $\theta\left(x_{0}, \ldots, x_{n}\right)$ consistent with $T(\mathcal{T})$. By quantifier elimination, we may assume that $\theta$ is quantifier-free, and hence that it is a disjunction of conjunctions of literals. Since any disjunction is implied by each of its disjuncts, and $\theta$ has at least one disjunct that is consistent with $T(\mathcal{T})$, we may in fact assume that $\theta$ is a conjunction of literals. Since we are working in a language with only unary relation symbols, we can split $\theta$ into formulas $\theta_{0}\left(x_{0}\right), \ldots, \theta_{n}\left(x_{n}\right)$ consistent with $T(\mathcal{T})$, and it suffices to show that each $\theta_{i}$ is implied over $T(\mathcal{T})$ by an atom of $T(\mathcal{T})$. Since each $\theta_{i}\left(x_{i}\right)$ is a conjunction of literals consistent with $T(\mathcal{T})$, there are $\sigma_{0}, \ldots, \sigma_{n} \in \mathcal{T}$ such that each $P^{\sigma_{i}}\left(x_{i}\right)$ logically implies $\theta_{i}\left(x_{i}\right)$. Since $\mathcal{T}$ is strongly atomic, there are atoms $\tau_{0}, \ldots, \tau_{n}$ of $T$ such that $\tau_{i}$ extends $\sigma_{i}$. It is easy to check that each $P^{\tau_{i}}\left(x_{i}\right)$ is an atom of $T(\mathcal{T})$ and implies $\theta_{i}\left(x_{i}\right)$ over $T(\mathcal{T})$.

(3) From an enumeration of the principal types of $T(\mathcal{T})$, we can build an enumeration $p_{0}(x), p_{1}(x), \ldots$ of the principal 1-types of $T(\mathcal{T})$ in the variable $x$. Let 
$f_{i}(k)=1$ if $P_{k}(x) \in p_{i}$, and let $f_{i}(k)=0$ otherwise. Then it is easy to see that $f_{0}, f_{1}, \ldots$ is an enumeration of the isolated paths of $\mathcal{T}$.

Thus we can further restate AMT as "If $\mathcal{T}$ is a strongly atomic extendible tree, then there is a listing of the isolated paths of $\mathcal{T}$." If we weaken the hypothesis of this statement, we get a different principle:

ATT (Atomic Tree Theorem): If $\mathcal{T}$ is an atomic extendible tree, then there is a listing of the isolated paths of $\mathcal{T}$.

ATT implies AMT by definition. The finite axiom of choice for $\Pi_{1}^{0}$ properties, and hence $\mathrm{B} \Sigma_{2}^{0}$, implies that every atomic tree is strongly atomic. (We show in Appendix $\mathrm{B}$ that this statement is in fact equivalent to $\mathrm{B} \Sigma_{2}^{0}$.) Thus ATT is provable in $\mathrm{RCA}_{0}+\mathrm{B} \Sigma_{2}^{0}+\mathrm{AMT}$. It is easy to see that ATT is also provable in $\mathrm{RCA}_{0}+\Pi_{1}^{0} \mathrm{G}$. These two facts together imply that ATT is restricted $\Pi_{2}^{1}$-conservative over $\mathrm{RCA}_{0}$ and $\Pi_{1}^{1}$-conservative over $\mathrm{RCA}_{0}+\mathrm{B} \Sigma_{2}^{0}$. They also imply that ATT is strictly weaker than both $\Pi_{1}^{0} \mathrm{G}$ and $\mathrm{AMT}+\mathrm{B} \Sigma_{2}^{0}$.

We do not know whether AMT (or even AMT $+\Pi_{1}^{0} \mathrm{GA}$ ) implies ATT over $\mathrm{RCA}_{0}$. Assuming AMT does not imply ATT, ATT is strictly intermediate in strength between $\mathrm{AMT}$ and $\mathrm{AMT}+\mathrm{B} \Sigma_{2}^{0}$, which suggests the idea of "miniaturizing" ATT, as we did to $\Pi_{1}^{0} \mathrm{G}$ to obtain $\Pi_{1}^{0} \mathrm{GA}$, yielding a principle strictly weaker than $\mathrm{B} \Sigma_{2}^{0}$ but not provable in $\mathrm{RCA}_{0}$. We show in Appendix $\mathrm{B}$ that this idea can indeed be implemented. 



\section{CHAPTER 4}

\section{Defining Homogeneity}

In this chapter, we compare several classically equivalent definitions of homogeneity.

Definition 4.1. Let $\mathcal{M}$ be a model. By an $M$-tuple we mean a tuple of elements of $M$. For $M$-tuples $\bar{a}$ and $\bar{b}$, we write $\bar{a} \equiv \bar{b}$ to mean that $\bar{a}$ and $\bar{b}$ have the same type. (If the language of $\mathcal{M}$ does not include equality, then the definition of $\equiv$ should also include the requirement that $\left(a_{0}, \ldots, a_{n}\right) \equiv\left(b_{0}, \ldots, b_{n}\right)$ implies that $a_{i}=a_{j}$ if and only if $b_{i}=b_{j}$.)

(i) A model $\mathcal{M}$ is 1-point homogeneous if for each pair of $M$-tuples $\bar{a} \equiv \bar{b}$ and each $c \in M$, there is a $d \in M$ such that $\bar{a} c \equiv \bar{b} d$.

(ii) A model $\mathcal{M}$ is 1-homogeneous if for each pair of $M$-tuples $\bar{a} \equiv \bar{b}$ and each $M$-tuple $\bar{c}$, there is an $M$-tuple $\bar{d}$ such that $\bar{a} \bar{c} \equiv \bar{b} \bar{d}$.

(iii) A model $\mathcal{M}$ is homogeneous if for each finite sequence of pairs of $M$-tuples $\bar{a}_{0} \equiv \bar{b}_{0}, \ldots, \bar{a}_{n} \equiv \bar{b}_{n}$ and each finite sequence of $M$-tuples $\bar{c}_{0}, \ldots, \bar{c}_{n}$, there are $M$-tuples $\bar{d}_{0}, \ldots, \bar{d}_{n}$ such that $\bar{a}_{i} \bar{c}_{i} \equiv \bar{b}_{i} \bar{d}_{i}$ for all $i \leqslant n$.

(iv) A model $\mathcal{M}$ is strongly 1-homogeneous if for each pair of $M$-tuples $\bar{a} \equiv \bar{b}$, there is an automorphism of $\mathcal{M}$ taking $\bar{a}$ to $\bar{b}$.

These definitions are easily seen to be classically equivalent by back and forth constructions. $\mathrm{ACA}_{0}$ is clearly powerful enough to carry out these constructions, so all of our definitions are equivalent in $\mathrm{ACA}_{0}$. However, as we show in this chapter, in $\mathrm{RCA}_{0}$ the situation is different. Of course, in $\mathrm{RCA}_{0}$ we do have (iii) $\Rightarrow$ (ii) $\Rightarrow$ (i), as well as (iv) $\Rightarrow$ (ii). We now consider the remaining implications. Table 4.1 summarizes our results. The entry in row $R$ and column $C$ describes the strength of the principle stating that every model with property $R$ has property $C$, in terms of a principle equivalent to it over $\mathrm{RCA}_{0}$.

Definition (i) is the standard one, but (iii) is better behaved than either (i) or (ii) from the reverse mathematical point of view. In particular, it is the one that yields a version of HMT equivalent to AMT, as shown in Theorem 7.6. By Theorem 4.3 below, the implication from (i) to (ii) is equivalent to $\mathrm{I} \Sigma_{2}^{0}$, and by Theorem 4.4, the implications from (ii) to (iii) and from (iv) to (iii) are both

TABLE 4.1. Versions of homogeneity

\begin{tabular}{|c|c|c|c|c|}
\hline & 1-pt. hom & 1-hom. & hom. & strongly 1-hom. \\
\hline 1-pt. hom & & $\mathrm{I} \Sigma_{2}^{0}$ & $\mathrm{I} \Sigma_{2}^{0}$ & $\mathrm{ACA}_{0}$ \\
\hline 1-hom. & $\mathrm{RCA}_{0}$ & & $\mathrm{~B}_{2}^{0}$ & $\mathrm{ACA}_{0}$ \\
\hline hom. & $\mathrm{RCA}_{0}$ & $\mathrm{RCA}_{0}$ & & $\mathrm{ACA}_{0}$ \\
\hline strongly 1-hom. & $\mathrm{RCA}_{0}$ & $\mathrm{RCA}_{0}$ & $\mathrm{~B} \Sigma_{2}^{0}$ & \\
\hline
\end{tabular}


equivalent to $\mathrm{B} \Sigma_{2}^{0}$. Since (i)-(iii) assert the existence of first order objects, while (iv) asserts the existence of a second order object (namely, an automorphism of $\mathcal{M}$ ), it is not surprising that the implications from (i)-(iii) to (iv) are at the level of $\mathrm{ACA}_{0}$. We have already noted that they all follow from $\mathrm{ACA}_{0}$. We now show that the weakest of them implies $\mathrm{ACA}_{0}$, using an example built by Hirschfeldt, Shore, and Slaman [23, Theorem 2.3] to show that the statement that any two atomic models of the same theory are isomorphic implies $\mathrm{ACA}_{0}$ over $\mathrm{RCA}_{0}$.

Proposition 4.2. The statement that every homogeneous model is strongly 1-homogeneous, i.e., (iii) $\Rightarrow$ (iv) in Definition 4.1, implies $\mathrm{ACA}_{0}$ over $\mathrm{RCA}_{0}$.

Proof. The example in [23] consists of two models, $\mathcal{A}$ and $\mathcal{B}$, in a language with unary relation symbols $R_{i}$ and $R_{i, j}$ for $i, j \in \mathbb{N}$, defined as follows. The domains of both $\mathcal{A}$ and $\mathcal{B}$ are $\mathbb{N}$ and in each the interpretation of $R_{i}$ is $\{\langle i, n\rangle \mid$ $n \in \mathbb{N}\}$. Let $\emptyset_{s}^{\prime}$ be the set of elements entering the halting set $\emptyset^{\prime}$ by stage $s$ of its enumeration. (The basic constructions of computability theory, including that of the sequence $\emptyset_{0}^{\prime}, \emptyset_{1}^{\prime}, \ldots$, can be carried out in $\mathrm{RCA}_{0}$; see for instance $[\mathbf{2 0}]$ or $[\mathbf{4 7}]$.) If $i \notin \emptyset_{s}^{\prime}$ then both interpretations of $R_{i, s}$ are empty. Suppose that $i$ enters $\emptyset^{\prime}$ at stage $t$. In $\mathcal{A}$ we put $\{i\} \times[0, t]$ into $R_{i, s}$ for each $s \geqslant t$, split the rest of $\{i\} \times \mathbb{N}$ into two computable infinite pieces, put one into $R_{i, s}$ for each $s \geqslant t$, and keep the other out of all the $R_{i, s}$. In $\mathcal{B}$ we keep $\{i\} \times[0, t]$ out of all the $R_{i, s}$, split the rest of $\{i\} \times \mathbb{N}$ into two computable infinite pieces, put one into $R_{i, s}$ for each $s \geqslant t$, and keep the other out of all the $R_{i, s}$.

We modify this example by adding a binary relation symbol $E$ to the language, and letting $\mathcal{M}$ be the model obtained by letting the interpretation of $E$ be an equivalence relation splitting the domain into two computable infinite equivalence classes, one of which is a copy of $\mathcal{A}$ and the other a copy of $\mathcal{B}$. To be precise, let $M=\mathbb{N}$ and let $E^{\mathcal{M}}(x, y)$ hold if and only if $x$ and $y$ are both even or both odd. Let $R_{i}(2 n)$ hold in $\mathcal{M}$ if and only if $R_{i}(n)$ holds in $\mathcal{A}$, let $R_{i}(2 n+1)$ hold in $\mathcal{M}$ if and only if $R_{i}(n)$ holds in $\mathcal{B}$, and act similarly for $R_{i, s}$.

A simple modification of the argument in the proof of Theorem 2.3 of $[\mathbf{2 3}]$ shows that $\mathcal{M}$ provably exists in $\mathrm{RCA}_{0}$. (All we need to add to the proof of quantifier elimination for the theory of $\mathcal{M}$ is a check of the consistency restrictions imposed by the axioms for the equivalence relation $E$, as discussed in Remark 2.3.) It is also easy to show in $\mathrm{RCA}_{0}$ that there are $a, b \in M$ such that $a \equiv b$ and $\neg E^{\mathcal{M}}(a, b)$, so if $\mathcal{M}$ is strongly 1 -homogeneous, then $\mathcal{A} \cong \mathcal{B}$, which implies $\mathrm{ACA}_{0}$, as shown in the proof of Theorem 2.3 of [23]. Thus it suffices to argue in $\mathrm{RCA}_{0}$ that $\mathcal{M}$ is homogeneous.

It is not difficult to see that, working in $\mathrm{RCA}_{0}$, we can define a function $f$ such that for each $a, x$, and $i<2$, we have $f(a, x, i)>x$ and $f(a, x, i) \equiv a$ (i.e., the elements $f(a, x, i)$ and $a$ of $\mathcal{M}$ have the same 1-type), and $E^{\mathcal{M}}(a, f(a, x, i))$ if and only if $i=1$.

Let $\bar{a}_{0} \equiv \bar{b}_{0}, \ldots, \bar{a}_{n} \equiv \bar{b}_{n}$ and $\bar{c}_{0}, \ldots, \bar{c}_{n}$ be $M$-tuples. For each $m \leqslant n$, proceed as follows. Let $\bar{a}_{m}=\left(a_{m, 0}, \ldots, a_{m, k}\right)$, let $\bar{b}_{m}=\left(b_{m, 0}, \ldots, b_{m, k}\right)$, and let $\bar{c}_{m}=$ $\left(c_{m, 0}, \ldots, c_{m, l}\right)$. For each $j \leqslant l$, proceed as follows. If $c_{m, j}=a_{m, j^{\prime}}$ for some $j^{\prime}$, then let $d_{m, j}=b_{m, j^{\prime}}$. Otherwise, let $x>b_{m, p}$ for all $p \leqslant k$. If $E^{\mathcal{M}}\left(a_{m, 0}, b_{m, 0}\right)$ then let $d_{m, j}=f\left(c_{m, j}, x, 1\right)$; otherwise, let $d_{m, j}=f\left(c_{m, j}, x, 0\right)$.

Let $\bar{d}_{m}=\left(d_{m, 0}, \ldots, d_{m, l}\right)$. It is easy to check that $\bar{a}_{m} \bar{c}_{m} \equiv \bar{b}_{m} \bar{d}_{m}$ for all $m \leqslant n$. Thus $\mathcal{M}$ is homogeneous. 
The remaining implications between our definitions of homogeneity are more subtle, involving induction and bounding principles.

THEOREM 4.3. The statement that every 1-point homogeneous model is 1homogeneous, i.e., (i) $\Rightarrow$ (ii) in Definition 4.1, is equivalent to $\mathrm{I}_{2}^{0}$ over $\mathrm{RCA}_{0}$.

Proof. Let $\mathcal{M}$ be 1 -point homogeneous. Let $\bar{a} \equiv \bar{b}$ and $\bar{c}=\left(c_{0}, \ldots, c_{n}\right)$ be $M$-tuples. For each $i \leqslant n$, the existence of a sequence $d_{0}, \ldots, d_{i-1}$ such that $\left(\bar{a}, c_{0}, \ldots, c_{i-1}\right) \equiv\left(\bar{b}, d_{0}, \ldots, d_{i-1}\right)$ is a $\Sigma_{2}^{0}$ property of $i$, so $\Sigma_{2}^{0}$-induction suffices to show that such sequences exist for all $i$, in particular $i=n$, which implies that $\mathcal{M}$ is 1-homogeneous.

We now argue in $\mathrm{RCA}_{0}$ to obtain the reversal. We use the equivalence between $\mathrm{I} \Sigma_{2}^{0}$ and finite $\Pi_{1}^{0}$-recursion mentioned in Section 2.1. Let $\varphi$ be a $\Pi_{1}^{0}$ formula defining a total function, and fix $z$ and $n$. We build a 1-point homogeneous model $\mathcal{M}$ such that if $\mathcal{M}$ is 1 -homogeneous then there is a sequence $x_{0}, \ldots, x_{n}$ with $x_{0}=z$ and $\varphi\left(x_{i}, x_{i+1}\right)$ for all $i<n$.

We work in the language with unary relation symbols $P_{i}$ for $i \in \mathbb{N}$ and a binary relation symbol $E$. Let $A$ be the following set of axioms, which we write informally, as formalizing them in our language is straightforward.

(1) $P_{i+1}(x) \rightarrow P_{i}(x)$ for all $i \in \mathbb{N}$.

(2) $E$ is an equivalence relation with two classes.

(3) Within each $E$-equivalence class, for each $i \in \mathbb{N}$ there are infinitely many $x$ such that $P_{i}(x) \wedge \neg P_{i+1}(x)$, and there are infinitely many $x$ such that $\neg P_{0}(x)$.

It is easy to show that $A$ admits effective quantifier elimination, as discussed at the end of Section 2.2: Fix an existential formula $(\exists y) \varphi(\bar{x} y)$, where $\varphi(\bar{x} y)$ is a conjunction of literals. Let $m$ be such that no $P_{i}$ with $i>m$ occurs in $\varphi$, and let $\mathcal{L}^{\prime}$ be the language consisting of $E$ and all $P_{i}$ with $i \leqslant m$. As mentioned in Remark 2.3 , we may assume that $\varphi$ is an $\mathcal{L}^{\prime}$-atomic diagram that includes $\neg x=y$ for all $x \in \bar{x}$ and is consistent with the assertion that $E$ is an equivalence relation. Let $\sim$ be the equivalence relation on the variables in $\bar{x} y$ defined by letting $u \sim v$ hold if and only if $\varphi$ contains $E(u, v)$. If $\sim$ has more than two equivalence classes, or $\varphi$ contains both $P_{j}(y)$ and $\neg P_{i}(y)$ for $i<j$, then $A \vdash(\exists y) \varphi(\bar{x} y) \leftrightarrow$ F. Otherwise, let $\psi$ be the result of removing from $\varphi$ all literals involving $y$ (and hence is just $\mathrm{T}$ if all literals in $\psi$ involve $y)$. Then it is easy to see that $A \vdash(\exists y) \varphi(\bar{x} y) \leftrightarrow \psi$.

We now specify a 1-point homogeneous model $\mathcal{M}$ of $A$. By effective quantifier elimination, it suffices to specify the atomic diagram of $\mathcal{M}$. Let $s_{0}, s_{1}, \ldots$ list all nonempty finite sequences of natural numbers with first element $z$. Let $\theta(k)$ hold if and only if, writing $s_{k}=\left(z=m_{0}, \ldots, m_{l-1}\right)$, we have $\varphi\left(m_{i}, m_{i+1}\right)$ for all $i<l-1$. Note that $\theta$ is $\Pi_{1}^{0}$, so there is a sequence of sets $R_{0} \supseteq R_{1} \supseteq \cdots$ such that $\theta(k)$ if and only if $k \in R_{i}$ for all $i$. We may assume that for each $i$, there are infinitely many $k$ such that $i$ is the least number for which $k \notin R_{i}$. Note also that if $\theta(k)$ and $\theta\left(k^{\prime}\right)$ both hold for $k \neq k^{\prime}$, then $s_{k}$ and $s_{k^{\prime}}$ must have different lengths, since $\varphi$ defines a function.

Let the domain $M$ of $\mathcal{M}$ be all numbers of the form $\langle i, j, k\rangle$ with $i, j \in\{0,1\}$ and $k \in \mathbb{N}$. Let $E^{\mathcal{M}}$ hold of two such numbers if and only if they have the same first coordinate. Let $P_{m}^{\mathcal{M}}(\langle i, 0, k\rangle)$ hold if and only if $k=\langle l, x\rangle$ for some $l>m$ and 
$x \in \mathbb{N}$. Let $P_{m}^{\mathcal{M}}(\langle 0,1, k\rangle)$ hold for all $k \in \mathbb{N}$. Let $P_{m}^{\mathcal{M}}(\langle 1,1, k\rangle)$ hold if and only if $k \in R_{m}$. Clearly, $\mathcal{M}$ is a model of $A$.

To see that $\mathcal{M}$ is 1 -point homogeneous, let

$$
\bar{a}=\left(a_{0}, \ldots, a_{m-1}\right) \equiv \bar{b}=\left(b_{0}, \ldots, b_{m-1}\right)
$$

be $M$-tuples and let $c \in M$. If $c=a_{i}$ for some $i<m$ then we can let $d=b_{i}$ and have $\bar{a} c \equiv \bar{b} d$, so we may assume that is not the case. Say that $\bar{a}$ and $\bar{b}$ are on opposite sides if there is an $i<m$ such that $a_{i}$ and $b_{i}$ have different first coordinates (in which case, the same must hold of all pairs $a_{i}, b_{i}$ ). Otherwise, say that $\bar{a}$ and $\bar{b}$ are on the same side. Let $l$ be large enough so that every number of the form $\langle i, j, x\rangle$ for $x>l$ is bigger than all the $b_{i}$. We have several cases.

(1) If $\bar{a}$ and $\bar{b}$ are on the same side and $c$ is of the form $\langle i, 0,\langle k, x\rangle\rangle$, then let $d=\left\langle i, 0,\left\langle k, x^{\prime}\right\rangle\right\rangle$ for some $x^{\prime}$ such that $\left\langle k, x^{\prime}\right\rangle>l$.

(2) If $\bar{a}$ and $\bar{b}$ are on the same side and $c$ is of the form $\langle 0,1, j\rangle$, then let $d=$ $\left\langle 0,1, j^{\prime}\right\rangle$ for some $j^{\prime}>l$.

(3) If $\bar{a}$ and $\bar{b}$ are on the same side, $c$ is of the form $\langle 1,1, j\rangle$, and $\theta(j)$ does not hold, then let $k$ be least such that $j \notin R_{k}$ and let $d=\langle 1,0,\langle k, x\rangle\rangle$ for some $x$ such that $\langle k, x\rangle>l$.

(4) If $\bar{a}$ and $\bar{b}$ are on opposite sides and $c$ is of the form $\langle i, 0,\langle k, x\rangle\rangle$, then let $d=\left\langle 1-i, 0,\left\langle k, x^{\prime}\right\rangle\right\rangle$ for some $x^{\prime}$ such that $\left\langle k, x^{\prime}\right\rangle>l$.

(5) If $\bar{a}$ and $\bar{b}$ are on opposite sides, $c$ is of the form $\langle 1,1, j\rangle$, and $\theta(j)$ holds, then let $d=\left\langle 0,1, j^{\prime}\right\rangle$ for some $j^{\prime}>l$.

(6) Finally, if $\bar{a}$ and $\bar{b}$ are on opposite sides and $c$ is of the form $\langle 0,1, j\rangle$, or $\bar{a}$ and $\bar{b}$ are on the same side, $c$ is of the form $\langle 1,1, j\rangle$, and $\theta(j)$ holds, then proceed as follows. Let $s$ be the longest string such that $s=s_{j^{\prime}}$ for some $j^{\prime}$ such that $\theta\left(j^{\prime}\right)$ and $b_{i}=\left\langle 1,1, j^{\prime}\right\rangle$ for some $i<m$, if there is such a string, and let $s$ be the empty string otherwise. (Note that we can pick out the set of $j^{\prime}$ such that $\theta\left(j^{\prime}\right)$ and $b_{i}=\left\langle 1,1, j^{\prime}\right\rangle$ for some $i<m$ by bounded $\Pi_{1}^{0}$-comprehension.) Then, by the hypothesis on $\varphi$, there is a string $s_{j^{\prime \prime}}$ of length $|s|+1$ such that $\theta\left(j^{\prime \prime}\right)$ holds. Let $d=\left\langle 1,1, j^{\prime \prime}\right\rangle$, and note that $d \neq b_{i}$ for all $i<m$.

In any case, it is easy to check that $\bar{a} c \equiv \bar{b} d$.

Now suppose that $\mathcal{M}$ is 1 -homogeneous. Since clearly $\langle 0,0,0\rangle \equiv\langle 1,0,0\rangle$, there exist $d_{0}, \ldots, d_{n-1}$ such that

$$
(\langle 0,0,0\rangle,\langle 0,1,0\rangle, \ldots,\langle 0,1, n-1\rangle) \equiv\left(\langle 1,0,0\rangle, d_{0}, \ldots, d_{n-1}\right) .
$$

We must have $d_{i}=\left\langle 1,1, k_{i}\right\rangle$ for each $i<n$. The numbers $k_{i}$ are all distinct and all satisfy $\theta$, so at least one $s_{k_{i}}$ has length at least $n$. This string witnesses the fact that the instance of $\Pi_{1}^{0}$-recursion corresponding to $\varphi, n$, and $z$ holds.

THEOREM 4.4. The following are equivalent over $\mathrm{RCA}_{0}$.

(1) $\mathrm{B} \Sigma_{2}^{0}$

(2) Every 1-homogeneous model is homogeneous, i.e., (ii) $\Rightarrow$ (iii) in Definition 4.1.

(3) Every strongly 1-homogeneous model is homogeneous, i.e., (iv) $\Rightarrow$ (iii) in Definition 4.1.

Proof. We first argue in $\mathrm{RCA}_{0}+\mathrm{B} \Sigma_{2}^{0}$ to prove (2), and hence (3). Let $\mathcal{M}$ be 1-homogeneous, let $\bar{a}_{0} \equiv \bar{b}_{0}, \ldots, \bar{a}_{n} \equiv \bar{b}_{n}$ be pairs of $M$-tuples, and let $\bar{c}_{0}, \ldots, \bar{c}_{n}$ be $M$-tuples. By 1-homogeneity, for each $i \leqslant n$, there is an $M$-tuple $\bar{d}$ such that 
$\bar{a}_{i} \bar{c}_{i} \equiv \bar{b}_{i} \bar{d}$. The condition that two given $M$-tuples have the same type is $\Pi_{1}^{0}$, so by the version of the finite axiom of choice mentioned in Section 2.1, there is a sequence of $M$-tuples $\bar{d}_{0}, \ldots, \bar{d}_{n}$ such that $\bar{a}_{i} \bar{c}_{i} \equiv \bar{b}_{i} \bar{d}_{i}$ for each $i \leqslant n$.

We now argue in $\mathrm{RCA}_{0}$ to show that (3) implies $\mathrm{B} \Sigma_{2}^{0}$, and hence so does (2). Assume that every strongly 1-homogeneous model is homogeneous. We show that $\mathrm{B} \Pi_{1}^{0}$ holds. (Recall that $\mathrm{B} \Pi_{1}^{0}$ and $\mathrm{B} \Sigma_{2}^{0}$ are equivalent over $\mathrm{RCA}_{0}$.) Let $\Psi$ be a $\Pi_{1}^{0}$ formula and $n$ a number such that $(\forall i<n)(\exists u) \Psi(i, u)$. We build a strongly 1-homogeneous model $\mathcal{M}$ such that if $\mathcal{M}$ is homogeneous then there is a $v$ for which $(\forall i<n)(\exists u<v) \Psi(i, u)$. Let $\Phi$ be a quantifier-free formula such that $\Psi(i, u) \equiv(\forall z) \Phi(i, u, z)$.

A first approach. Since our model is somewhat complicated, we begin by sketching a simpler version that does not prove the theorem but helps motivate our full construction. We work in the language with unary relation symbols $U_{i}$ for $i<n$ and $R_{i}$ for $i \in \mathbb{N}$, and a binary relation symbol $E$. Let $\widehat{A}$ be the following set of axioms.

(1) The $U_{i}$ partition the universe into pairwise disjoint sets.

(2) $E$ is an equivalence relation. If $E(x, y)$ holds then $U_{i}(x)$ and $U_{i}(y)$ both hold for some $i<n$, and $E$ splits each $U_{i}$ into two infinite classes.

(3) $R_{k+1}(x) \rightarrow R_{k}(x)$ for all $i \in \mathbb{N}$.

(4) For each $i<n$ and each $m \in \mathbb{N}$ there are infinitely many $x$ in each $E$ equivalence class of $U_{i}$ such that $R_{k}(x)$ holds if and only if $k<m$.

It is easy to show that $\widehat{A}$ admits effective quantifier elimination. Let $\mathcal{N}$ be the model of $\widehat{A}$ defined as follows. The universe $N$ of $\mathcal{N}$ consists of all numbers of the form $\langle i, j, m\rangle$ with $i<n, j \in\{0,1\}$, and $m \in \mathbb{N}$. Let $U_{i}^{\mathcal{N}}(x)$ hold if and only if the first coordinate of $x$ is $i$. Let $E^{\mathcal{N}}(x, y)$ hold if and only if $x$ and $y$ have the same first and second coordinates. For $j \in\{0,1\}$, let $R_{k}^{\mathcal{N}}(\langle i, j, 2 m\rangle)$ hold if and only if $k<m$. Let $R_{k}^{\mathcal{N}}(\langle i, 0,2 m+1\rangle)$ hold for all $k$. Let $R_{k}^{\mathcal{N}}(\langle i, 1,2\langle m, c\rangle+1\rangle)$ hold if and only if $(\exists u<m)(\forall z<k) \Phi(i, u, z)$.

Note that $(\forall k)(\exists u<m)(\forall z<k) \Phi(i, u, z) \leftrightarrow(\exists u<m) \Psi(i, u)$. (The right-toleft-direction is obvious. For the other direction, suppose that $(\forall u<m) \neg \Psi(i, u)$. Then for each $u<m$ there is a $z_{u}$ such that $\neg \Phi\left(i, u, z_{u}\right)$. These $z_{u}$ can be found effectively, so their maximum $k$ exists. Then $(\forall u<m)(\exists z<k+1) \neg \Phi(i, u, z)$.)

Suppose $\mathcal{N}$ is homogeneous. Let $a_{i}=\langle i, 0,0\rangle$, let $b_{i}=\langle i, 1,0\rangle$, and let $c_{i}=$ $\langle i, 0,1\rangle$. Then $a_{i} \equiv b_{i}$ for all $i<n$, so there are $d_{0}, \ldots, d_{n-1}$ such that $a_{i} c_{i} \equiv b_{i} d_{i}$ for all $i<n$. It follows from the definition of $\mathcal{N}$ and the remark in the previous paragraph that each $d_{i}$ is of the form $\left\langle i, 1,2\left\langle m_{i}, c\right\rangle+1\right\rangle$ for some $m_{i}$ such that $\left(\exists u<m_{i}\right) \Psi(i, u)$. Let $m=\max _{i<n} m_{i}$. Then $(\forall i<n)(\exists u<m) \Psi(i, u)$.

However, we cannot show that $\mathcal{N}$ is strongly 1 -homogeneous, or even 1-homogeneous. Indeed, if we assume that $\mathcal{N}$ is 1 -homogeneous, then we can argue as above to obtain $m_{0}, \ldots, m_{n-1}$, because $\left(a_{0}, \ldots, a_{n-1}\right) \equiv\left(b_{0}, \ldots, b_{n-1}\right)$, so there are $d_{0}, \ldots, d_{n-1}$ such that

$$
\left(a_{0}, \ldots, a_{n-1}, c_{0}, \ldots, c_{n-1}\right) \equiv\left(b_{0}, \ldots, b_{n-1}, d_{0}, \ldots, d_{n-1}\right) .
$$

To get around this problem, we introduce new binary relations between elements of different $U_{i}$ 's. The goal is to ensure that if elements $\left(a_{0}, \ldots, a_{n-1}\right) \equiv\left(b_{0}, \ldots, b_{n-1}\right)$ 
of a model $\mathcal{M}$ can be used to produce $m_{0}, \ldots, m_{n-1}$ using 1-homogeneity as above, then these sequences already contain enough information to obtain $m_{0}, \ldots, m_{n-1}$ without appealing to 1-homogeneity. More generally, we want to ensure that if $\left(a_{0}, \ldots, a_{k-1}\right) \equiv\left(b_{0}, \ldots, b_{k-1}\right)$ then these sequences contain enough information to obtain an automorphism of $\mathcal{M}$ taking each $a_{i}$ to $b_{i}$.

Axioms. We work in the language with unary relation symbols $U_{i}$ for $i<n$ and $R_{i}$ for $i \in \mathbb{N}$, and binary relation symbols $E$ and $Q_{i}$ for $i \in \mathbb{N}$. Let $A$ be the following set of axioms, some of which we write informally, as formalizing them in our language is straightforward. The first three families of axioms are the same as above. The fourth ensures that the $Q_{i}$ hold only between elements of different $U_{j}$ 's. The fifth expresses the fact that any existential statement with parameters that is not forbidden by the other axioms holds. This last axiom is necessary to ensure that $A$ admits effective quantifier elimination.

(1) The $U_{i}$ partition the universe into pairwise disjoint sets.

(2) $E$ is an equivalence relation. If $E(x, y)$ holds then $U_{i}(x)$ and $U_{i}(y)$ both hold for some $i<n$, and $E$ splits each $U_{i}$ into two infinite classes.

(3) $R_{i+1}(x) \rightarrow R_{i}(x)$ for all $i \in \mathbb{N}$.

(4) If $Q_{i}(x, y)$ holds then $U_{j}(x)$ and $U_{j^{\prime}}(y)$ hold for some $j \neq j^{\prime}$.

(5) Let $x_{0}, \ldots, x_{k-1}$ be distinct elements and let $i_{0}, \ldots, i_{k-1}$ be such that $U_{i_{j}}\left(x_{j}\right)$ holds for all $j<k$. Let

$$
F_{0}, \ldots, F_{k-1}, \widehat{F}_{0}, \ldots, \widehat{F}_{k-1}, G_{0}, \ldots, G_{k-1}, \widehat{G}_{0}, \ldots, \widehat{G}_{k-1}
$$

be finite sets such that $F_{j} \cap \widehat{F}_{j}=G_{j} \cap \widehat{G}_{j}=\emptyset$ for all $j<k$. Then for each $i<n$ and each $m \in \mathbb{N}$, there are infinitely many $y$ in each $E$-equivalence class of $U_{i}$ such that

(a) $\neg R_{m}(y)$ and, if $m>0$, also $R_{m-1}(y)$;

(b) for each $j<k$ such that $i_{j} \neq i$, we have $Q_{d}\left(x_{j}, y\right)$ for all $d \in F_{j}$ and $\neg Q_{d}\left(x_{j}, y\right)$ for all $d \in \widehat{F}_{j}$; and

(c) for each $j<k$ such that $i_{j} \neq i$, we have $Q_{d}\left(y, x_{j}\right)$ for all $d \in G_{j}$ and $\neg Q_{d}\left(y, x_{j}\right)$ for all $d \in \widehat{G}_{j}$.

Quantifier elimination. To show that $A$ admits effective quantifier elimination, as discussed at the end of Section 2.2, fix a formula $(\exists y) \varphi(\bar{x} y)$, where $\varphi(\bar{x} y)$ is a conjunction of literals. Let $m$ be such that no $R_{i}$ or $Q_{i}$ with $i>m$ occurs in $\varphi$, and let $\mathcal{L}^{\prime}$ be the language consisting of $E$, all $U_{i}$ with $i<n$, and all $R_{i}$ and $Q_{i}$ with $i \leqslant m$. As mentioned in Remark 2.3, we may assume that $\varphi$ is an $\mathcal{L}^{\prime}$-atomic diagram that is consistent with the assertion that $E$ is an equivalence relation, and includes $\neg u=v$ for all distinct $u, v \in \bar{x} y$.

If both $U_{i}(u)$ and $U_{j}(u)$ for $j \neq i$ occur in $\varphi$, or $\neg U_{i}(u)$ occurs in $\varphi$ for all $i<n$, then $A \vdash(\exists y) \varphi(\bar{x} y) \leftrightarrow \mathrm{F}$. So we may assume that we can partition $\bar{x} y$ into classes $C_{0}, \ldots, C_{n-1}$ such that if $u \in C_{i}$ then $U_{i}(u)$ occurs in $\varphi$ and $\neg U_{j}(u)$ occurs in $\varphi$ for all $j \neq i$ with $j<n$. Suppose that at least one of the following conditions holds.

(1) Both $R_{i}(u)$ and $\neg R_{j}(u)$ for $j<i$ occur in $\varphi$.

(2) $E(u, v)$ occurs in $\varphi$, and $u \in C_{i}$ and $u \in C_{j}$ for $i \neq j$.

(3) $Q_{i}(u, v)$ occurs in $\varphi$, and $u, v \in C_{j}$ for some $j<n$. 
(4) Let $\sim$ be the equivalence relation on the variables in $\bar{x} y$ defined by letting $u \sim v$ hold if and only if $\varphi$ contains $E(u, v)$. Then there is an $i<n$ such that the restriction of $\sim$ to $C_{i}$ has more than two equivalence classes.

Then $A \vdash(\exists y) \varphi(\bar{x} y) \leftrightarrow \mathrm{F}$. Otherwise, application of the fifth axiom family of $A$ shows that $A \vdash(\exists y) \varphi(\bar{x} y) \leftrightarrow \theta(\bar{x})$, where $\theta$ is the result of removing from $\varphi$ all literals involving $y$ (and hence is just $\mathrm{T}$ if all literals in $\varphi$ involve $y$ ).

Thus the deductive closure $T$ of $A$ exists. We build a model $\mathcal{M}$ of $A$ below, thus showing $T$ is a theory. First we describe and name some of the types of $T$.

Types. By effective quantifier elimination, the types of $T$ can be identified with atomic types.

The 1-types of $T$ in a given variable $x$ are then of two kinds. We have the principal type $r_{i, j}(x)$ for $i<n$ and $j \in \mathbb{N}$, which is determined by $U_{i}(x)$ and $\neg R_{j}(x)$, and, if $j>0$, also $R_{j-1}(x)$. We also have the type $r_{i, \infty}(x)$, determined by $U_{i}(x)$ and $R_{j}(x)$ for all $j \in \mathbb{N}$. Let $r_{i, \infty / m}(x)$ be the type determined by $U_{i}(x)$ and $R_{j}(x)$ if and only if $(\exists u<m)(\forall z<j) \Phi(i, u, z)$. Note that $r_{i, \infty / m}=r_{i, \infty}$ if $(\exists u<m) \Psi(i, u)$ holds, and otherwise $r_{i, \infty / m}=r_{i, k}$ for some $k \in \mathbb{N}$. We use this type in the same way as in our simplified model $\mathcal{N}$ above.

There are uncountably many 2 -types of $T$, but we are interested in only countably many of them. The 2-types $p(x, y)$ that include both $U_{i}(x)$ and $U_{i}(y)$ are all realized in the model $\mathcal{M}$ defined below, but we do not need to refer to them directly. To describe the other 2-types in given variables $x$ and $y$ realized in $\mathcal{M}$, we first define the partial 2-type $s_{i, j, S}(x, y)$ for $i<n$ and $j \in \mathbb{N} \cup\{\infty\}$, and a finite or cofinite set $S$. This type states that $x$ has type $r_{i, j}$ and $Q_{m}(x, y)$ holds if and only if $m \in S$. If $S$ is finite then we say that a 2-type extending this partial type is $i$-finitary. If $S$ is cofinite then we say that a 2-type extending this partial type is $i$-infinitary. Now let $s_{i_{0}, i_{1}, j_{0}, j_{1}, S_{0}, S_{1}}\left(x_{0}, x_{1}\right)$ for $i_{0} \neq i_{1}$ be the 2 -type determined by $s_{i_{0}, j_{0}, S_{0}}\left(x_{0}, x_{1}\right) \cup s_{i_{1}, j_{1}, S_{1}}\left(x_{1}, x_{0}\right)$.

We get around the deficiency of our simplified model $\mathcal{N}$ above by ensuring that in $\mathcal{M}$, if $x_{0}, x_{1}$ are not large enough to encode an $m$ such that $\Psi(i, u)$ holds for some $u<m$, and we have $U_{i}\left(x_{0}\right), U_{i}\left(x_{1}\right)$, and $\neg E\left(x_{0}, x_{1}\right)$, then for some $l \in\{0,1\}$, whenever $U_{j}(y)$ holds for $j \neq i$, the 2-type of $\left(x_{l}, y\right)$ is $i$-finitary, while the 2-type of $\left(x_{1-l}, y\right)$ is $i$-infinitary. To implement this strategy, we use the following definitions.

Let $s_{i, j, S / \text { fin }_{d}^{c}}(x, y)$ be the partial 2-type stating that $x$ has type $r_{i, j}$ and $Q_{k}(x, y)$ holds if and only if $k \in S$ and either $k<c$ or $(\exists u<d)(\forall z<k) \Phi(i, u, z)$. Then $s_{i, j, S / \text { fin }_{d}^{c}}=s_{i, j, S}$ if $\Psi(i, u)$ holds for some $u<d$, and otherwise $s_{i, j, S / \text { find }_{d}^{c}}=s_{i, j, S^{\prime}}$ for some finite set $S^{\prime}$. (Of course, if $S$ is finite, we could have $S^{\prime}=S$.)

Let $s_{i, j, S / \inf _{d}^{c}}(x, y)$ be the partial 2-type stating that $x$ has type $r_{i, j}$ and $Q_{k}(x, y)$ holds if and only if $k \in S$ or both $k>c$ and $(\forall u<d)(\exists z<k) \neg \Phi(i, u, z)$. Then $s_{i, j, S / \text { inf }_{d}^{c}}=s_{i, j, S}$ if $\Psi(i, u)$ holds for some $u<d$, and otherwise $s_{i, j, S / \inf _{d}^{c}}=s_{i, j, S^{\prime}}$ for some cofinite set $S^{\prime}$. (Of course, if $S$ is cofinite, we could have $S^{\prime}=S$.)

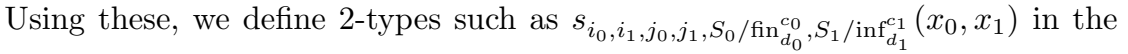
obvious way.

For each of the 2-types we have defined, if $j, j_{0}$, or $j_{1}$ is $\infty$, we also consider the corresponding type with $\infty$ replaced by $\infty / m$ as in the definition of $r_{i, \infty} / m$. For instance, the type $s_{i_{0}, i_{1}, \infty / m, j_{1}, S_{0}, S_{1}}\left(x_{0}, x_{1}\right)$ is defined in the same way as the type $s_{i_{0}, i_{1}, \infty, j_{1}, S_{0}, S_{1}}\left(x_{0}, x_{1}\right)$, except that $x_{0}$ now has type $r_{i_{0}, \infty / m}$ instead of $r_{i_{0}, \infty}$.

Each type of $T$ can be specified by listing its 2-subtypes. Note in particular that, by quantifier elimination, for tuples $\left(a_{0}, \ldots, a_{m}\right)$ and $\left(b_{0}, \ldots, b_{m}\right)$ of elements 
of the model $\mathcal{M}$ built below, if $a_{i} a_{j} \equiv b_{i} b_{j}$ for all $i, j \leqslant m$, then $\left(a_{0}, \ldots, a_{m}\right) \equiv$ $\left(b_{0}, \ldots, b_{m}\right)$.

The model $\mathcal{M}$. We now specify a strongly 1 -homogeneous model $\mathcal{M}$ of $A$. By effective quantifier elimination, it suffices to specify the atomic diagram of $\mathcal{M}$.

The domain $M$ of $\mathcal{M}$ consists of all numbers of the form $\langle i, j, l, m\rangle$ with $i<n$, $j \in\{0,1\}$, and $l, m \in \mathbb{N}$. For $a \in M$ and $k \in\{1,2,3,4\}$, let $\pi_{k}(a)$ be the $k$ th coordinate of $a$.

Let $U_{i}^{\mathcal{M}}(a)$ hold if and only if $\pi_{1}(a)=i$. Let $E^{\mathcal{M}}(a, b)$ hold if and only if $\pi_{1}(a)=\pi_{1}(b)$ and $\pi_{2}(a)=\pi_{2}(b)$. Let $R_{k}^{\mathcal{M}}(\langle i, j, l, 2\langle m, c\rangle\rangle)$ hold if and only if $k<m$. Let $R_{k}^{\mathcal{M}}(\langle i, 0, l, 2 m+1\rangle)$ hold for all $k$. Let $R_{k}^{\mathcal{M}}(\langle i, 1, l, 2\langle m, c\rangle+1\rangle)$ hold if and only if $(\exists u<m)(\forall z<k) \Phi(i, u, z)$. (We assume our pairing function is onto.)

In other words, we give $\langle i, j, l, 2\langle m, c\rangle\rangle$ type $r_{i, m}$, give $\langle i, 0, l, 2 m+1\rangle$ type $r_{i, \infty}$, and give $\langle i, 1, l, 2\langle m, c\rangle+1\rangle$ type $r_{i, \infty} / m$. This definition ensures that for each $i$, there is an $m_{i}$ such that all elements of the form $\left\langle i, 1, l, 2\left\langle m_{i}, c\right\rangle+1\right\rangle$ have type $r_{i, \infty}$, but any sequence $m_{0}, \ldots, m_{n-1}$ of such elements can be used to satisfy our instance of $\mathrm{B \Pi}_{1}^{0}$.

In defining the interpretations of the $Q_{k}$, the idea is to use elements with third coordinate $l$ to provide witnesses for the satisfaction of particular 1-types over elements with third coordinates less than $l$. In particular, we provide witnesses to the satisfaction of the fifth axiom group of $A$, though we have to do more than that, since we have to realize $i$-infinitary types while preserving strong 1-homogeneity. To implement the strategy described above for repairing the problem with our previous model $\mathcal{N}$, we also wish to ensure that the following property holds. Let $x, y$ have different first coordinates. Let $i=\pi_{1}(x)$ and $m=\pi_{4}(x)$. Suppose that $\neg \Psi(i, u)$ for all $u<m$. If $\pi_{2}(x)=0$ then the type of $(x, y)$ is $i$-finitary, and otherwise this type is $i$-infinitary.

We first define the $Q_{k}$ on pairs of elements with the same third coordinate. For each $k, l \in \mathbb{N}$, let $Q_{k}^{\mathcal{M}}\left(\langle i, j, l, m\rangle,\left\langle i^{\prime}, j^{\prime}, l, m^{\prime}\right\rangle\right)$ hold if and only if $i^{\prime} \neq i$ and $j=1$. That is, we give each pair $\left(\langle i, 0, l, m\rangle,\left\langle i^{\prime}, j^{\prime}, l, m^{\prime}\right\rangle\right)$ with $i^{\prime} \neq i$ a type extending a partial type of the form $s_{i, d, \emptyset}$ (which is $i$-finitary), and each pair $\left(\langle i, 1, l, m\rangle,\left\langle i^{\prime}, j^{\prime}, l, m^{\prime}\right\rangle\right)$ with $i^{\prime} \neq i$ a type extending a partial type of the form $s_{i, d, \infty}$ (which is $i$-infinitary).

Now let $l>0$. Partition the elements of $M$ of the form $\langle i, 0, l, 2 m+1\rangle$ into infinite sets $B_{i, 0,0}, B_{i, 0,1}, \ldots$ Let $m \in \mathbb{N}$. Partition the elements of $M$ of the form $\langle i, 0, l, 2\langle m, c\rangle\rangle$ into infinite sets $B_{i, 3 m+1,0}, B_{i, 3 m+1,1}, \ldots$, partition the elements of $M$ of the form $\langle i, 1, l, 2\langle m, c\rangle\rangle$ into infinite sets $B_{i, 3 m+2,0}, B_{i, 3 m+2,1}, \ldots$, and partition the elements of $M$ of the form $\langle i, 1, l, 2\langle m, c\rangle+1\rangle$ into infinite sets $B_{i, 3 m+3,0}, B_{i, 3 m+3,1}, \ldots$ Assign to each $x \in \mathbb{N}$

(1) a tuple $\left(a_{0}, \ldots, a_{k-1}\right)$ of elements of $M$ with third coordinates less than $l$,

(2) a tuple of numbers

$$
\left(d_{0}, \ldots, d_{k-1}, d_{0}^{\prime}, \ldots, d_{k-1}^{\prime}, c_{0}, \ldots, c_{k-1}, c_{0}^{\prime}, \ldots, c_{k-1}^{\prime}\right)
$$

and

(3) a tuple of finite or cofinite sets $\left(S_{0}, \ldots, S_{k-1}, S_{0}^{\prime}, \ldots, S_{k-1}^{\prime}\right)$ (by which we mean that some of these sets might be finite while others might be cofinite).

We call this 3-tuple, consisting of a tuple of elements, a tuple of numbers, and a tuple of sets, the parameters associated with $x$. Perform this assignment in such a way that each set of parameters is associated with some $x$. 
Fix $x \in \mathbb{N}$ and the parameters associated with $x$ as above. Fix $i<n$. Let $a_{k} \in \bigcup_{j \in \mathbb{N}} B_{i, j, x}$. Let $i_{j}=\pi_{1}\left(a_{j}\right)$, let $e_{j}=\pi_{2}\left(a_{j}\right)$, and let $m_{j}=\pi_{4}\left(a_{j}\right)$. For $j<k$, let $t_{j}$ be such that $a_{j}$ has been given type $r_{i_{j}, t_{j}}$. Thus $t_{j}$ could be a natural number, $\infty$, or $\infty / q$ for some $q \in \mathbb{N}$. For $j<k$, if $e_{j}=0$ then let $\bmod _{j}$ denote $\operatorname{fin}_{\min \left(d_{j}, m_{j}\right)}^{c_{j}}$, and otherwise let $\bmod _{j}$ denote $\inf _{\min \left(d_{j}, m_{j}\right)}^{c_{j}}$. If $e_{k}=0$ then let $\bmod _{k}$ denote $\operatorname{fin}_{\min \left(d_{j}^{\prime}, m_{k}\right)}^{c_{j}^{\prime}}$, and otherwise let $\bmod _{k}$ denote $\inf _{\min \left(d_{j}^{\prime}, m_{k}\right)}^{c_{j}^{\prime}}$.

For each $j<k$ such that $i_{j} \neq i_{k}$, define the $Q_{c}^{\mathcal{M}}$ on $\left(a_{j}, a_{k}\right)$ and $\left(a_{k}, a_{j}\right)$ so

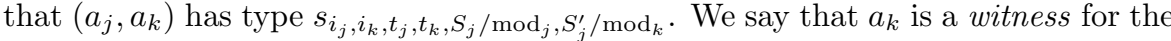
tuple $\left(a_{0}, \ldots, a_{k-1}\right)$, as well as for the whole set of parameters associated with $x$.

For any $a \in M$ with $\pi_{3}(a)<l$ that is not among $a_{0}, \ldots, a_{k-1}$, let $Q_{c}^{\mathcal{M}}\left(a, a_{k}\right)$ hold if and only if $\pi_{1}(a) \neq i_{k}$ and $\pi_{2}(a)=1$, and let $Q_{c}^{\mathcal{M}}\left(a_{k}, a\right)$ hold if and only if $\pi_{1}(a) \neq i_{k}$ and $e_{k}=1$.

We have completed the definition of $\mathcal{M}$. It is clear that $\mathcal{M}$ satisfies the first four axiom groups of $A$. It also satisfies the fifth axiom group because for all elements $a_{0}, \ldots, a_{k-1}$ of $M$ and all finite sets

$$
F_{0}, \ldots, F_{k-1}, \widehat{F}_{0}, \ldots, \widehat{F}_{k-1}, G_{0}, \ldots, G_{k-1}, \widehat{G}_{0}, \ldots, \widehat{G}_{k-1}
$$

we eventually assign elements $a_{k}$ as in the previous paragraph. The mod operation in the definition of the type of $\left(a_{j}, a_{k}\right)$ is not a problem if the $c_{j}$ are sufficiently large (i.e., larger than all numbers mentioned in the particular instance of the fifth axiom group we are considering).

Type translation. To show that $\mathcal{M}$ is strongly 1 -homogeneous, we first need to discuss the process of translating types between the equivalence classes of a given $U_{i}^{\mathcal{M}}$, which will help us define the required automorphism of $\mathcal{M}$. Consider the type

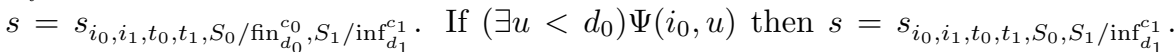
In this case, let $\widehat{S}_{0}=S_{0}$. Otherwise, there is a finite set $\widehat{S}_{0}$ such that $s=$

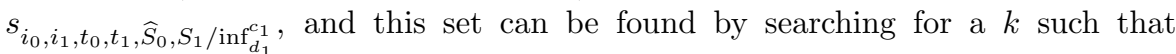
$\left(\forall u<d_{0}\right)(\exists z<k) \neg \Phi\left(i_{0}, u, z\right)$. In either case, if $u$ is least such that $\Psi\left(i_{0}, u\right)$

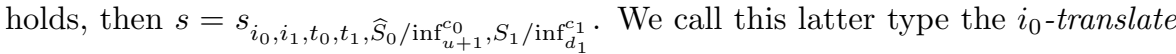
of $s$. We call $\left(u+1, d_{1}, \widehat{S}_{0}, S_{1}\right.$, inf, inf) the conditions of this translate. To find this translate, we need to be able to determine $\widehat{S}_{0}$ and $u$. The former can be obtained effectively from $u$, though, so in fact, all we need is $u$.

Similarly, if we know the least $v$ such that $\Psi\left(i_{1}, v\right)$, then we can find the $i_{1^{-}}$

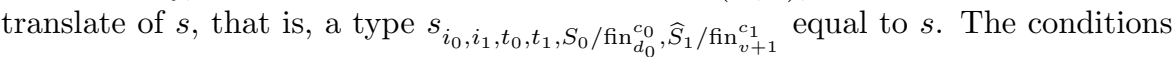
of this translate are $\left(d_{0}, v+1, S_{0}, \widehat{S_{1}}\right.$, fin, fin). If we know both $u$ and $v$, then we can also find the $i_{0}, i_{1}$-translate of $s$, that is, a type $s_{i_{0}, i_{1}, t_{0}, t_{1}, \widehat{S}_{0} / \mathrm{inf}_{u+1}^{c_{0}}, \widehat{S}_{1} / \mathrm{fin}_{v+1}^{c_{1}}}$ equal to $s$. The conditions of this translate are $\left(u+1, v+1, \widehat{S}_{0}, \widehat{S_{1}}\right.$, inf, fin). The same

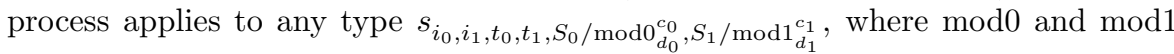
are each either fin or inf.

For $I \subseteq n$, the $I$-translate of a type $s$ as above is $s$ if $i_{0}, i_{1} \notin I$, the $i_{l}$-translate of $s$ if $i_{l} \in I$ and $i_{1-l} \notin I$, and the $i_{0}, i_{1}$-translate of $s$ if $i_{0}, i_{1} \in I$.

Strong 1-homogeneity. We now show that $\mathcal{M}$ is strongly 1-homogeneous. Let $\left(a_{0}, \ldots, a_{m-1}\right) \equiv\left(b_{0}, \ldots, b_{m-1}\right)$. We need to build an automorphism $f$ of $\mathcal{M}$ permuting these $M$-tuples. 
Let $I$ be the set of all $i$ for which there is a $j<m$ such that $\pi_{1}\left(a_{j}\right)=i$ (and hence $\pi_{1}\left(b_{j}\right)=i$ ) and $\pi_{2}\left(a_{j}\right) \neq \pi_{2}\left(b_{j}\right)$. (Note that then every $a_{j^{\prime}}, b_{j^{\prime}}$ with first coordinate in $I$ have different second coordinates.) It obviously has to be the case that if $f(a)=b$ and $\pi_{1}(a) \in I$, then $\pi_{2}(a) \neq \pi_{2}(b)$. We build $f$ so that it is also the case that if $f(a)=b$ and $\pi_{1}(a) \notin I$, then $\pi_{2}(a)=\pi_{2}(b)$. Say that $\left(a_{0}, \ldots, a_{r-1}\right) \equiv\left(b_{0}, \ldots, b_{r-1}\right)$ is an $I$-pair if $I$ is the set of all $i$ for which there is a $j<r$ such that $\pi_{1}\left(a_{j}\right)=i$ and $\pi_{2}\left(a_{j}\right) \neq \pi_{2}\left(b_{j}\right)$. Thus our starting $M$-tuples form an $I$-pair.

We in fact show the following: Let $\left(a_{0}, \ldots, a_{r-1}\right) \equiv\left(b_{0}, \ldots, b_{r-1}\right)$ be an $I$-pair, and let $a_{r} \in M$. Then we can effectively find $b_{r} \in M$ such that $\left(a_{0}, \ldots, a_{r}\right) \equiv$ $\left(b_{0}, \ldots, b_{r}\right)$ is an $I$-pair. (In other words, we can define a function taking each $I$-pair $\left(a_{0}, \ldots, a_{r-1}\right) \equiv\left(b_{0}, \ldots, b_{r-1}\right)$ and $a_{r} \in M$ to an appropriate $\left.b_{r}.\right)$

This fact suffices to build $f$ by the usual back and forth argument: Let $f\left(a_{i}\right)=$ $b_{i}$ for $i<m$. Then let $a_{m}$ be the least element of $M$ not among the $a_{i}$ for $i<m$, find $b_{m}$ such that $\left(a_{0}, \ldots, a_{m}\right) \equiv\left(b_{0}, \ldots, b_{m}\right)$ is an $I$-pair, and let $f\left(a_{m}\right)=b_{m}$. Then let $b_{m+1}$ be the least element of $M$ not among the $b_{i}$ for $i \leqslant m$, find $a_{m+1}$ such that $\left(a_{0}, \ldots, a_{m+1}\right) \equiv\left(b_{0}, \ldots, b_{m+1}\right)$ is an $I$-pair (which we can do because the notion of $I$-pair is a symmetric relation on tuples), and let $f\left(a_{m+1}\right)=b_{m+1}$. Continue in this way to build an automorphism $f$ of $\mathcal{M}$.

So fix an $I$-pair $\left(a_{0}, \ldots, a_{r-1}\right) \equiv\left(b_{0}, \ldots, b_{r-1}\right)$ and $a_{r} \in M$. We show how to obtain a $b_{r}$ as above. As pointed out above, to show that $\left(a_{0}, \ldots, a_{r}\right) \equiv\left(b_{0}, \ldots, b_{r}\right)$, it is enough to show that $a_{i} a_{r} \equiv b_{i} b_{r}$ for all $i<r$. If $a_{r}=a_{j}$ for some $j<r$, then we can let $b_{r}=b_{j}$, so we suppose this is not the case.

We begin with the following observation. Suppose $a, b \in M$ have different first coordinates, and let $i$ be the first coordinate of $a$. Suppose further that the fourth coordinate of $a$ is a number $m$ such that $(\forall u<m) \neg \Psi(i, u)$. It is easy to check that the construction of $\mathcal{M}$ ensures that if the second coordinate of $a$ is 0 then the 2-type of $(a, b)$ is $i$-finitary, while if the second coordinate of $a$ is 1 then the 2-type of $(a, b)$ is $i$-infinitary. Now suppose that $a b \equiv c d$ are pairs of elements of $M$ such that $a$ and $b$ have different first coordinates, and $a$ and $c$ have different second coordinates. Let $i$ be the first coordinate of $a$ (and hence of $c$ ) and let $m$ be the maximum of the fourth coordinates of $a$ and $c$. Then there is a $u<m$ such that $\Psi(i, u)$, as otherwise one of the 2-types of $(a, b)$ and $(c, d)$ would be $i$-finitary, while the other would be $i$-infinitary.

We now claim there is a sequence $\left(u_{i}\right)_{i \in I}$ such that $u_{i}$ is the least number for which $\Psi\left(i, u_{i}\right)$ holds. If all the $a_{j}$ with $j<r$ have the same first coordinate, then $|I| \leqslant 1$, so the claim is true by hypothesis (together with bounded $\Pi_{1}^{0}$-comprehension). Otherwise, fix $i \in I$, let $j$ be such that $\pi_{1}\left(a_{j}\right)=i$ and $\pi_{2}\left(a_{j}\right) \neq \pi_{2}\left(b_{j}\right)$, and let $j^{\prime}$ be such that $\pi_{1}\left(a_{j^{\prime}}\right) \neq i$. Since $a_{j} a_{j^{\prime}} \equiv b_{j} b_{j^{\prime}}$, the observation of the previous paragraph implies that, letting $m_{i}$ be the maximum of the fourth coordinates of $a_{j}$ and $b_{j}$, there is a $u<m_{i}$ such that $\Psi(i, u)$. By bounded $\Pi_{1}^{0}$-comprehension (with the bound being $\max _{i \in I} m_{i}$ ), we can find $\left(u_{i}\right)_{i \in I}$. As discussed above, this fact means that we can determine the $I$-translate of any type of

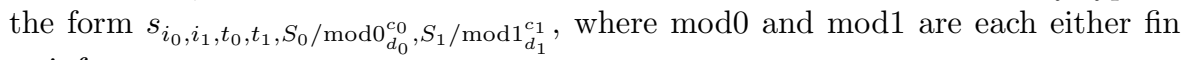
or inf.

We begin the process of determining $b_{r}$ by ensuring that it has the same 1-type as $a_{r}$. To do so, we define a set $D$ from which $b_{r}$ is drawn. We ensure that every element of $D$ has the same 1-type as $a_{r}$, and has the same second coordinate as $a_{r}$ 
if and only if $\pi_{1}\left(a_{r}\right) \notin I$. We also ensure that $D$ contains witnesses for every set of parameters in the definition of $\mathcal{M}$. (Recall the definition of witnessing a set of parameters from the section of this proof in which we defined the model $\mathcal{M}$.)

Let $i=\pi_{1}\left(a_{r}\right)$, let $j=\pi_{2}\left(a_{r}\right)$, and let $k=\pi_{4}\left(a_{r}\right)$. Let $D$ be the set of all elements of $M$ of the form $\left\langle i, j^{\prime}, l, k^{\prime}\right\rangle$ where $l \in \mathbb{N}$ and $j^{\prime}, k^{\prime}$ are as follows. If $i \notin I$ then $j^{\prime}=j$; otherwise $j^{\prime}=1-j$. If $k$ is of the form $2\langle m, c\rangle$, then $k^{\prime}$ is of the form $2\langle m, d\rangle$. If $i \notin I, j=0$, and $k$ is odd, then $k^{\prime}$ is odd. If $i \notin I, j=1$, and $k$ is of the form $2\langle m, c\rangle+1$, then $k^{\prime}$ is of the form $2\langle m, d\rangle+1$. If $i \in I, j=0$, and $k$ is odd, then $k^{\prime}$ is of the form $2\left\langle u_{i}+1, d\right\rangle+1$. If $i \in I, j=1$, and $k$ is of the form $2\langle m, c\rangle+1$ with $m \leqslant u_{i}$, then $k^{\prime}$ is of the form $2\langle q, d\rangle$ where $q$ is least such that $(\forall u<m)(\exists z<q) \neg \Phi(i, u, z)$ (which must exist by the definition of $\left.u_{i}\right)$. If $i \in I$, $j=1$, and $k$ is of the form $2\langle m, c\rangle+1$ with $m>u_{i}$, then $k^{\prime}$ is odd.

It is easy to check that $D$ has the properties mentioned above. The fact that we choose $b_{r}$ from $D$ is enough to ensure that $a_{j} a_{r} \equiv b_{j} b_{r}$ if $\pi_{1}\left(a_{j}\right)=\pi_{1}\left(a_{r}\right)$, and that if $\left(a_{0}, \ldots, a_{r}\right) \equiv\left(b_{0}, \ldots, b_{r}\right)$ then these tuples form an $I$-pair.

Let $V$ be the set of $j<r$ such that $\pi_{1}\left(a_{j}\right) \neq \pi_{1}\left(a_{r}\right)$, and let $W$ be set of $j \in V$ such that either $a_{r}$ is a witness for a tuple that includes $a_{j}$ or $a_{j}$ is a witness for a tuple that includes $a_{r}$. Let $P$ be the set of all expressions that are either in $\mathbb{N}$, equal to $\infty$, or equal to $\infty / m$ for some $m \in \mathbb{N}$ (i.e., all expressions $t$ such that $r_{i, t}$ is one of our named 1-types).

If $j \in W$ then from the construction of $\mathcal{M}$ we can determine $t, t^{\prime} \in P$, finite or cofinite sets $S$ and $S^{\prime}$, numbers $d, d^{\prime}, c, c^{\prime}$, and expressions mod0 and mod1, each equal to either fin or inf, so that $\left(a_{j}, a_{r}\right)$ has type $s_{\pi_{1}\left(a_{j}\right), \pi_{1}\left(a_{r}\right), t, t^{\prime}, S / \bmod 0_{d}^{c}, S^{\prime} / \bmod 1_{d^{\prime}}^{c^{\prime}}}$. Let $\left(d_{j}, d_{j}^{\prime}, \widehat{S}_{j}, \widehat{S}_{j}^{\prime}, \bmod 0 j, \bmod 1 j\right)$ be the conditions of the $I$-translate of this type. Let $c_{j}=c$ and $c_{j}^{\prime}=c^{\prime}$.

If $j \in V \backslash W$ then from the construction of $\mathcal{M}$ we can determine $t, t^{\prime} \in P$ and sets $S$ and $S^{\prime}$, each equal to either $\emptyset$ or $\mathbb{N}$, such that $\left(a_{j}, a_{r}\right)$ has type $s=$ $s_{\pi_{1}\left(a_{j}\right), \pi_{1}\left(a_{r}\right), t, t^{\prime}, S, S^{\prime}}$. If $S=\emptyset$ then let $\bmod 0$ be fin, and otherwise let $\bmod 0$ be inf. If $S^{\prime}=\emptyset$ then let mod1 be fin, and otherwise let $\bmod 1$ be inf. Then $s=s_{\pi_{1}\left(a_{j}\right), \pi_{1}\left(a_{r}\right), t, t^{\prime}, S / \bmod 0_{0}^{0}, S^{\prime} / \bmod 1_{0}^{0}}$. Let $\left(d_{j}, d_{j}^{\prime}, \widehat{S}_{j}, \widehat{S}_{j}^{\prime}, \bmod 0 j, \bmod 1 j\right)$ be the conditions of the $I$-translate of this type. Let $c_{j}=c_{j}^{\prime}=0$.

Now consider the tuples

$$
\left(b_{j}\right)_{j \in V},\left(d_{j}\right)_{j \in V}\left(d_{j}^{\prime}\right)_{j \in V}\left(c_{j}\right)_{j \in V}\left(c_{j}^{\prime}\right)_{j \in V}, \text { and }\left(S_{j}\right)_{j \in V}\left(S_{j}^{\prime}\right)_{j \in V} .
$$

At some point in the construction of $\mathcal{M}$, witnesses for this set of parameters are provided. It is easy to check that there is such a witness $b_{r}$ that is taken from $D$. By the definition of the $I$-translate, we have $a_{j} a_{r} \equiv b_{j} b_{r}$ for all $j \in V$. As mentioned above, we also have $a_{j} a_{r} \equiv b_{j} b_{r}$ for all $j<r$ that are not in $V$. Thus $\left(a_{0}, \ldots, a_{r}\right) \equiv\left(b_{0}, \ldots, b_{r}\right)$, which, as mentioned above, implies that these tuples form an $I$-pair.

Using homogeneity. Finally, suppose $\mathcal{M}$ is homogeneous. Let $a_{i}=\langle i, 0,0,0\rangle$, let $b_{i}=\langle i, 1,0,0\rangle$, and let $c_{i}=\langle i, 0,0,1\rangle$. Then $a_{i} \equiv b_{i}$ for all $i<n$, so there are $d_{0}, \ldots, d_{n-1}$ such that $a_{i} c_{i} \equiv b_{i} d_{i}$ for all $i<n$. Since the type of $c_{i}$ is $r_{i, \infty}$, each $d_{i}$ is of the form $\left\langle i, 1, l_{i}, 2\left\langle m_{i}, k_{i}\right\rangle+1\right\rangle$ for some $m_{i}$ such that $\left(\exists u<m_{i}\right) \Psi(i, u)$. Then $\max _{i<n} m_{i}$ witnesses the satisfaction of our instance of $\mathrm{B} \Pi_{1}^{0}$.

One of the important facts about homogeneity is that atomic, prime, and saturated models are homogeneous. The standard proof of this fact carries through in 
$\mathrm{ACA}_{0}$ for any of our definitions of homogeneity. In $\mathrm{RCA}_{0}$, we have the following version. (Recall the definition of strong saturation from Definition 2.1.)

Proposition 4.5. The following are provable in $\mathrm{RCA}_{0}$.

(1) Every atomic model is homogeneous.

(2) Every prime model is homogeneous.

(3) Every saturated model is 1-homogeneous.

(4) Every strongly saturated model is homogeneous.

Proof. (1) Let $\mathcal{M}$ be atomic, and let $\bar{a}_{0} \equiv \bar{b}_{0}, \ldots, \bar{a}_{n} \equiv \bar{b}_{n}$ and $\bar{c}_{0}, \ldots, \bar{c}_{n}$ be $M$-tuples. There is a formula $\psi\left(\bar{x}_{0} \bar{y}_{0} \ldots \bar{x}_{n} \bar{y}_{n}\right)$ isolating the type of $\bar{a}_{0} \bar{c}_{0} \ldots \bar{a}_{n} \bar{c}_{n}$. Then for each $i \leqslant n$, the formula

$$
\varphi_{i}\left(\bar{x}_{i} \bar{y}_{i}\right) \equiv\left(\exists \bar{x}_{0} \bar{y}_{0} \ldots \bar{x}_{i-1} \bar{y}_{i-1} \bar{x}_{i+1} \bar{y}_{i+1} \ldots \bar{x}_{n} \bar{y}_{n}\right) \psi\left(\bar{x}_{0} \bar{y}_{0} \ldots \bar{x}_{n} \bar{y}_{n}\right)
$$

isolates the type of $\bar{a}_{i} \bar{c}_{i}$. For each $i \leqslant n$, we have $\mathcal{M} \vDash(\exists \bar{y}) \varphi_{i}\left(\bar{a}_{i} \bar{y}\right)$, so $\mathcal{M} \vDash$ $(\exists \bar{y}) \varphi_{i}\left(\bar{b}_{i} \bar{y}\right)$, and thus there are $M$-tuples $\bar{d}_{0}, \ldots, \bar{d}_{n}$ such that $\mathcal{M} \vDash \varphi_{i}\left(\bar{b}_{i}, \bar{d}_{i}\right)$ for each $i \leqslant n$. Then $\bar{a}_{i} \bar{c}_{i} \equiv \bar{b}_{i} \bar{d}_{i}$ for each $i \leqslant n$.

(2) Hirschfeldt, Shore, and Slaman [23] noted that the proof that every prime model is atomic carries through in $\mathrm{RCA}_{0}$.

(3) Let $\mathcal{M}$ be saturated, and let $\bar{a} \equiv \bar{b}$ and $\bar{c}$ be $M$-tuples. Let $\Gamma=\{\psi(\bar{b} \bar{y})$ : $\mathcal{M} \vDash \psi(\bar{a} \bar{c})\}$. Then $\Gamma$ is a type of the theory of $(\mathcal{M}, \bar{b})$, so it is realized by some $M$-tuple $\bar{d}$. Then $\bar{a} \bar{c} \equiv \bar{b} \bar{d}$.

(4) Let $\mathcal{M}$ be strongly saturated, and let $\bar{a}_{0} \equiv \bar{b}_{0}, \ldots, \bar{a}_{n} \equiv \bar{b}_{n}$ and $\bar{c}_{0}, \ldots, \bar{c}_{n}$ be $M$-tuples. Let $\Gamma=\left\{\psi\left(\bar{b}_{i} \bar{y}_{i}\right): i \leqslant n \wedge \mathcal{M} \vDash \psi\left(\bar{a}_{i} \bar{c}_{i}\right)\right\}$. Then $\Gamma\left(\bar{y}_{0} \ldots \bar{y}_{n}\right)$ is a partial type of the theory of $(\mathcal{M}, \bar{b})$, so it is realized by some $M$-tuple $\bar{d}_{0} \ldots \bar{d}_{n}$. Then $\bar{a}_{i} \bar{c}_{i} \equiv \bar{b}_{i} \bar{d}_{i}$ for each $i \leqslant n$.

Proposition 4.5, combined with Theorem 4.4, implies that $\mathrm{B} \Sigma_{2}^{0}$ suffices to show that every saturated model is homogeneous. Theorem C.1 below shows that $\mathrm{WKL}_{0}$ also suffices to prove this statement, which implies that the statement is $\Pi_{1}^{1}$-conservative over $\mathrm{RCA}_{0}$, and hence in particular does not imply $\mathrm{B} \Sigma_{2}^{0}$. We do not, however, know the exact strength of this statement.

It is straightforward to argue as in [23] to show that the model $\mathcal{M}$ defined in the proof of Proposition 4.2 is atomic. Thus we have the following fact.

Proposition 4.6. The statement that every atomic model is strongly 1-homogeneous is equivalent to $\mathrm{ACA}_{0}$ over $\mathrm{RCA}_{0}$.

We do not know the exact strengths of the statements that every prime model is strongly 1-homogeneous, and that every (strongly) saturated model is strongly 1-homogeneous

Another important property of homogeneous models is that they are completely determined by their type spectra. That is, two homogeneous models with the same type spectra are isomorphic. The following result shows that, for any of our definitions of homogeneity, this principle is equivalent to $\mathrm{ACA}_{0}$.

Proposition 4.7. The following are equivalent over $\mathrm{RCA}_{0}$.

(1) $\mathrm{ACA}_{0}$

(2) If $\mathcal{M}$ and $\mathcal{N}$ are 1-point homogeneous and realize the same types, then $\mathcal{M} \cong$ $\mathcal{N}$

(3) If $\mathcal{M}$ and $\mathcal{N}$ are homogeneous and realize the same types, then $\mathcal{M} \cong \mathcal{N}$. 
(4) If $\mathcal{M}$ and $\mathcal{N}$ are strongly 1-homogeneous and realize the same types, then $\mathcal{M} \cong \mathcal{N}$.

Proof. $\mathrm{ACA}_{0}$ is powerful enough to carry out the classical back and forth construction needed to prove the statements above, and (2) clearly implies (3) and (4), so we are left with showing that (3) and (4) imply $\mathrm{ACA}_{0}$.

Let $\mathcal{A}$ and $\mathcal{B}$ be the models from $[\mathbf{2 3}]$ described in the proof of Proposition 4.2. As shown in $[\mathbf{2 3}], \mathcal{A}$ and $\mathcal{B}$ are both atomic models of the same theory, and hence realize the same types. By Proposition $4.5, \mathcal{A}$ and $\mathcal{B}$ are homogeneous. Let $\left(a_{0}, \ldots, a_{n}\right) \equiv\left(b_{0}, \ldots, b_{n}\right) \in A$. Let $f$ be the function defined by $f\left(a_{i}\right)=b_{i}$, $f\left(b_{i}\right)=a_{i}$, and $f(x)=x$ for all other $x$. Then $f$ is an automorphism of $\mathcal{A}$, since the language of $\mathcal{A}$ is unary. Thus $\mathcal{A}$ is also strongly 1-homogeneous. Similarly, $\mathcal{B}$ is also strongly 1-homogeneous. As shown in $[\mathbf{2 3}]$, the existence of an isomorphism between $\mathcal{A}$ and $\mathcal{B}$ implies $\mathrm{ACA}_{0}$. 



\section{CHAPTER 5}

\section{Closure Conditions and Model Existence}

In this chapter, we consider various closure conditions on sets of types. Recall that, in the reverse mathematical setting, an infinite set of types is coded as a list by a single second order object (see Definition 2.2). Suppose we have defined such a listing of types $X=\left\{p_{i}\right\}_{i \in \mathbb{N}}$. When we refer to a finite sequence $q_{0}, \ldots, q_{n}$ of types in $X$, we always think of this set as encoded by a finite sequence $i_{0}, \ldots, i_{n}$ of natural numbers such that $q_{k}=p_{i_{k}}$ for all $k \leqslant n$, and similarly for finite sets of types in $X$.

\subsection{Type spectra of homogeneous models}

As mentioned above, Goncharov [15] and Peretyat'kin [41] characterized the sets of types that are type spectra of homogeneous models. We say that types $p_{0}, \ldots, p_{n}$ are basically consistent if whenever $p_{i}$ and $p_{j}$ share a tuple of variables $\bar{x}$, we have $p_{i}\left\lceil\bar{x}=p_{j}\lceil\bar{x}\right.$. A formula $\varphi(\bar{x} \bar{y})$ is consistent with a type $p(\bar{x})$ if $(\exists \bar{y}) \varphi \in p$.

Theorem 5.1 (Goncharov [15] and Peretyat'kin [41]). Let $T$ be a theory, and let $X \subset S(T)$ be countable. Then $X$ is the type spectrum of a homogeneous model if and only if it satisfies the following conditions.

(1) $T \in X$.

(2) $X$ is closed under variable substitutions, i.e., if $p(\bar{x}) \in X$ and $|\bar{y}|=|\bar{x}|$ then $p(\bar{y}) \in X$.

(3) $X$ is closed under taking subtypes.

(4) Extension: If $p(\bar{x}) \in X$ and $\varphi(\bar{x} \bar{y})$ is consistent with $p$, then there is a $q(\bar{x} \bar{y}) \in X$ such that $p \cup\{\varphi\} \subseteq q$.

(5) Pairwise Full Type Amalgamation: For each basically consistent $p_{1}, p_{2} \in$ $X$, there is a $q \in X$ such that $p_{1} \cup p_{2} \subseteq q$.

Remark 5.2. Suppose that $X$ satisfies (1)-(4) above, and let $p\left(x_{0}, \ldots, x_{m-1}\right) \in$ $X$. Let $f: n \rightarrow m$ be a function, and let $q\left(x_{0}, \ldots, x_{m-1}, y_{0}, \ldots, y_{n-1}\right)$ be the unique type of $T$ containing $p$ and $y_{i}=x_{f(i)}$ for all $i<n$. Then a simple application of extension shows that $q \in X$. However, this application requires our assumption that the language of $T$ includes equality. If we did not make this assumption, we would want to extend the definition of closure under variable substitutions slightly to say that if $p \in X$ and $q$ are as above, then $q \in X$. The only place below where this issue matters is in the proof of Lemma 6.9, which is used in the proof of Theorem 6.10 .

We name the above set of closure conditions.

Definition 5.3. An $X \subset S(T)$ satisfies the pairwise full amalgamation closure conditions if $X$ satisfies all the conditions in Theorem 5.1. 
We study effective versions of the extension condition (4) below, but first we define various other versions of type amalgamation and study their relationships. We begin with the notion of finite full type amalgamation, which is classically equivalent to pairwise full type amalgamation. Basic consistency, in general, is not enough to guarantee that finite sets of types can be amalgamated in general. We can have types $p(x, y), q(x, z), r(y, z)$ such that $p\lceil x=q \uparrow x, p \uparrow y=r \uparrow y$, and $q \uparrow z=r \uparrow z$, yet $p, q, r$ cannot be amalgamated at all (i.e., there is no type of the given theory that amalgamates them). For example, consider a graph with four elements $a, b, c, d$, with edges from $a$ to $c$ and $b$ to $d$, and no other edges. Let $p(x, y)$ be the type of $a, b$; let $q(x, z)$ be the type of $a, c$, which is the same as the type of $b, d$; and let $r(y, z)$ be the same as $q(x, z)$ with $x$ substituted by $y$.

We say that types $q_{0}\left(\bar{y}_{0}\right), \ldots, q_{n}\left(\bar{y}_{n}\right)$ are consistent if they are basically consistent and there is an $i \leqslant n$ such that all the shared variables are in $\bar{y}_{i}$. (That is, we cannot have $z \in \bar{y}_{j} \cap \bar{y}_{k}$ for $j \neq k$ unless $z \in \bar{y}_{i}$.) This condition ensures that this finite set of types can be amalgamated in the classical setting. We modify condition 5, pairwise full type amalgamation, as follows.

Finite Full Type Amalgamation: For each consistent set of types $q_{0}, \ldots, q_{n} \in$ $X$, there is a $q \in X$ such that $q_{0} \cup \cdots \cup q_{n} \subseteq q$.

Note that, as mentioned at the beginning of this chapter, we think of finite subsets of $X$ as coded by indices, so the above is in fact a first order statement, as are other similar statements considered below. (That is, the universal quantification over sets of types $q_{0}, \ldots, q_{n} \in X$ is really over finite sets of indices of elements of $X=\left\{p_{i}\right\}_{i \in \mathbb{N}}$, and the existential quantification over $q \in X$ is really over indices of elements of $X$.)

As with 1-homogeneity and homogeneity, the reason that pairwise amalgamation does not immediately yield finite amalgamation when working in $\mathrm{RCA}_{0}$ is that the property of being an amalgamator of a set of types is $\Pi_{1}^{0}$.

Suppose $X=\left\{p_{i}\right\}_{i \in \mathbb{N}}$ satisfies finite full type amalgamation. Let $i_{0}, \ldots, i_{n} \in \mathbb{N}$. For each $k \leqslant n$, let $q_{k}$ be a (not necessarily proper) subtype of a type obtained from $p_{i_{k}}$ by variable substitution. Suppose that $q_{0}, \ldots, q_{n}$ form a consistent set of types. It is in the spirit of the notion of finite type amalgamation that there should be a type in $X$ amalgamating $q_{0}, \ldots, q_{n}$. However, even in the presence of the first four conditions in Theorem 5.1, it might not be provable in $\mathrm{RCA}_{0}$ that this is indeed the case, because there might not be a sequence $j_{0}, \ldots, j_{n} \in \mathbb{N}$ such that $p_{j_{k}}=q_{k}$ for all $k \leqslant n$. Again, the issue here is that having $p_{j_{k}}=q_{k}$ is a $\Pi_{1}^{0}$ property. To avoid this problem, we introduce the following condition: $X=\left\{p_{i}\right\}_{i \in \mathbb{N}}$ is closed under sequences of variable substitutions if for every sequence $i_{0}, \ldots, i_{n} \in \mathbb{N}$ and every sequence of types $q_{0}, \ldots, q_{n}$ such that $q_{k}$ is a (not necessarily proper) subtype of a type obtained from $p_{i_{k}}$ by variable substitution, there is a sequence $j_{0}, \ldots, j_{n} \in \mathbb{N}$ such that $p_{j_{k}}=q_{k}$ for all $k \leqslant n$.

REMARK 5.4. If $X$ satisfies the first four conditions in Theorem 5.1, then it is provable in $\mathrm{RCA}_{0}+\mathrm{B} \Sigma_{2}^{0}$ that $X$ is closed under sequences of variable substitutions. (The proof is a straightforward application of the finite axiom of choice for $\Pi_{1}^{0}$ properties.)

DeFInItion 5.5. An $X \subset S(T)$ satisfies the finite full amalgamation closure conditions if $X$ satisfies the conditions in Theorem 5.1 with pairwise full type 
amalgamation replaced by finite full type amalgamation, and is also closed under sequences of variable substitutions.

Pairwise full type amalgamation is to 1-homogeneity as finite full type amalgamation is to homogeneity, an analogy that is made more precise below. We also consider a weaker version of pairwise full type amalgamation, which corresponds to 1-point homogeneity.

1-Point Full Type Amalgamation: For each basically consistent pair of types $p_{1}(\bar{x} y), p_{2}(\bar{x} z) \in X$, there is a $q \in X$ such that $p_{1} \cup p_{2} \subseteq q$.

DeFinition 5.6. An $X \subset S(T)$ satisfies the 1-point full amalgamation closure conditions if $X$ satisfies the conditions in Theorem 5.1 with pairwise full type amalgamation replaced by 1-point full type amalgamation.

\subsection{Type spectra of general models}

Theorem 5.1 characterizes the sets of types that are type spectra of homogeneous models. As noted by Peretyat'kin [41], it can easily be modified to characterize the sets of types that are type spectra of models in general.

TheOREM 5.7 (Peretyat'kin [41]). Let $T$ be a theory, and let $X \subset S(T)$ be countable. Then $X$ is the type spectrum of a model if and only if it satisfies the following conditions (the first four of which are the same as in Theorem 5.1).

(1) $T \in X$.

(2) $X$ is closed under variable substitutions.

(3) $X$ is closed under taking subtypes.

(4) Extension: If $p(\bar{x}) \in X$ and $\varphi(\bar{x} \bar{y})$ is consistent with $p$, then there is a $q(\bar{x} \bar{y}) \in X$ such that $p \cup\{\varphi\} \subseteq q$.

(5) Pairwise Free Type Amalgamation: For each $p_{1}, p_{2} \in X$ whose variables do not overlap, there is a $q \in X$ such that $p_{1} \cup p_{2} \subseteq q$.

As in the full amalgamation case, we have the following notion, classically equivalent to pairwise free type amalgamation.

Finite Free Type Amalgamation: For each finite set of types $p_{0}, \ldots, p_{n} \in X$ whose variables do not overlap, there is a $q \in X$ such that $p_{0} \cup \cdots \cup p_{n} \subseteq q$.

DEFINITION 5.8. An $X \subset S(T)$ satisfies the pairwise free amalgamation closure conditions if it satisfies all the conditions in Theorem 5.7; and it satisfies the finite free amalgamation closure conditions if it satisfies these conditions with pairwise free type amalgamation replaced by finite free type amalgamation, and is also closed under sequences of variable substitutions.

Remark 5.9. Suppose that $X$ satisfies the finite free amalgamation closure conditions. Then $X$ satisfies a "finite sequence" version of the extension condition (condition (4) in Theorems 5.1 and 5.7), in the following sense. We first describe a notation that is also useful below. When we write $\varphi(\bar{x})$ for a formula $\varphi$, to express the fact that free variables of $\varphi$ are among the ones in the tuple $\bar{x}$, we subsequently write $\varphi[\bar{y}]$, where $\bar{y}=\left(y_{0}, \ldots, y_{n}\right)$ is a tuple of variables of the same length as $\bar{x}$, for the formula obtained by replacing each free occurrence of $x_{i}$ by $y_{i}$. We also adopt the analogous notation for types. Let $p_{0}\left(\bar{x}_{0}\right), \ldots, p_{n}\left(\bar{x}_{n}\right) \in X$ and let $\varphi_{0}\left(\bar{x}_{0} \bar{y}_{0}\right), \ldots, \varphi_{n}\left(\bar{x}_{n} \bar{y}_{n}\right)$ be formulas such that each $\varphi_{k}$ is consistent with $p_{k}$. Let $\bar{z}_{0}, \ldots, \bar{z}_{n}$ and $\bar{w}_{0}, \ldots, \bar{w}_{n}$ be pairwise disjoint tuples of variables such that 
each $\bar{z}_{i}$ has the same arity as $\bar{x}_{i}$, and each $\bar{w}_{i}$ has the same arity as $\bar{y}_{i}$. Then we can argue in $\mathrm{RCA}_{0}$ as follows. The sequence $p_{0}\left[\bar{z}_{0}\right], \ldots, p_{n}\left[\bar{z}_{n}\right]$, which exists as a sequence of elements of $X$ by closure under sequences of variable substitutions, is amalgamated by some $q \in X$. The formula $\varphi=\bigwedge_{k \leqslant n} \varphi_{k}\left[\bar{z}_{k} \bar{w}_{k}\right]$ is consistent with $q$, so there is an $r \supseteq q \cup\{\varphi\}$ in $X$. Thus there are $r_{0}, \ldots, r_{n} \in X$ such that $p_{k}\left[\bar{z}_{k}\right] \cup\left\{\varphi_{k}\left[\bar{z}_{k} \bar{w}_{k}\right]\right\} \subseteq r_{k}$ for each $k \leqslant n$, which implies that there are $s_{0}, \ldots, s_{n} \in X$ such that $p_{k}\left(\bar{x}_{k}\right) \cup\left\{\varphi_{k}\left(\bar{x}_{k} \bar{y}_{k}\right)\right\} \subseteq s_{k}$ for each $k \leqslant n$. (The existence of the sequence $r_{0}, \ldots, r_{n}$ and that of the sequence $s_{0}, \ldots, s_{n}$ both follow by closure under sequences of variable substitutions.)

\subsection{Comparing closure conditions}

We study the reverse mathematics of Theorems 5.1 and 5.7 below, but first we discuss the relationships between the closure conditions introduced in this chapter. Note that if $X$ satisfies the pairwise full amalgamation closure conditions, then $X$ satisfies the pairwise free amalgamation conditions, and similarly for the finite versions. We also highlight the following obvious fact for future reference. (Note that, in the reverse mathematical setting, a set of types is encoded as a list of types, as in Definition 2.2. Two lists of types $\left\{p_{i}\right\}_{i \in \mathbb{N}}$ and $\left\{q_{i}\right\}_{i \in \mathbb{N}}$ can be equal as sets of types, meaning that for each $i$ there is a $j$ such that $q_{j}=p_{i}$ and for each $i$ there is a $j$ such that $p_{j}=q_{i}$, but have different closure properties. It is worth keeping in mind, for instance, that when working in a model of second order arithmetic with nonstandard first order part, the fact that $\left\{p_{i}\right\}_{i \in \mathbb{N}}$ and $\left\{q_{i}\right\}_{i \in \mathbb{N}}$ are equal as sets of types does not necessarily imply that for every sequence $i_{0}, \ldots, i_{n}$ there is a sequence $j_{0}, \ldots, j_{n}$ such that $q_{j_{k}}=p_{i_{k}}$ for all $k \leqslant n$.)

Proposition 5.10. The following is provable in $\mathrm{RCA}_{0}$. If the lists of types $X$ and $Y$ are equal as sets of types and $X$ satisfies the pairwise (free or full) type amalgamation condition, then so does $Y$, and similarly for 1-point full amalgamation, and each of the first four conditions in Theorem 5.1.

We show in Theorem 5.16 that this proposition does not hold for finite amalgamation.

The difference between the 1-point, pairwise, and finite versions of our closure conditions turns out to be $\mathrm{I} \Sigma_{2}^{0}$. We record the full result here, although its proof depends on two results proved in the next chapter.

THEOREM 5.11. The following are equivalent over $\mathrm{RCA}_{0}$.

(1) $\mathrm{I} \Sigma_{2}^{0}$

(2) If a list of types satisfies the 1-point full amalgamation closure conditions then it satisfies the pairwise full amalgamation closure conditions.

(3) If a list of types satisfies the pairwise full amalgamation closure conditions then it satisfies the finite full amalgamation closure conditions.

(4) If a list of types satisfies the pairwise free amalgamation closure conditions then it satisfies the finite free amalgamation closure conditions.

(5) If a list of types satisfies the pairwise full amalgamation closure conditions then it satisfies the finite free amalgamation closure conditions.

Proof. To show that (1) implies (2), let $X$ be a list of types satisfying the 1point full amalgamation closure conditions. Let $p\left(\bar{x}, y_{0}, \ldots, y_{m}\right)$ and $q\left(\bar{x}, z_{0}, \ldots, z_{n}\right)$ be basically consistent elements of $X$. By assumption, there is a type $r_{0}\left(\bar{x}, y_{0}, z_{0}\right)$ 
amalgamating $p\left\lceil\bar{x} y_{0}\right.$ and $q \uparrow \bar{x} z_{0}$. Then there are types $r_{0}^{\prime}\left(\bar{x}, y_{0}, z_{0}, y_{1}\right)$ amalgamating $p\left\lceil\bar{x} y_{0} y_{1}\right.$ and $r_{0}$, and $r_{0}^{\prime \prime}\left(\bar{x}, y_{0}, z_{0}, z_{1}\right)$ amalgamating $q \uparrow \bar{x} z_{0} z_{1}$ and $r_{0}$. Hence there is a type $r_{1}\left(\bar{x}, y_{0}, z_{0}, y_{1}, z_{1}\right)$ amalgamating $r_{0}^{\prime}$ and $r_{0}^{\prime \prime}$. By finite $\Pi_{1}^{0}$-recursion (which, as mentioned in Section 2.1, is provable from $\mathrm{I} \Sigma_{2}^{0}$ ), we can continue in this fashion to eventually obtain a type amalgamating $p$ and $q$.

To show that (1) implies (3), and hence that (1) implies (5), let $X$ be a list of types satisfying the pairwise full amalgamation closure conditions, and let $q_{0}, \ldots, q_{n} \in X$ be a consistent sequence of types. By the definition of consistency, we may assume that all free variables shared by two or more of the $q_{i}$ are among the free variables of $q_{0}$. Let $P$ be the property that holds of $i \leqslant n$ if and only if there is a $q \in X$ such that $q_{0} \cup \cdots \cup q_{i} \subseteq q$. It is easy to check that $P$ is $\Sigma_{2}^{0}$. Furthermore, $P(0)$ holds, and if $P(i)$ holds for $i<n$, then, letting $q$ be the witness to this fact, $q, q_{i+1}$ are consistent, so there is an $r \in X$ such that $q_{0} \cup \cdots \cup q_{i} \subseteq q \cup q_{i+1} \subseteq r$; that is, $P(i+1)$ holds. Thus, by $\mathrm{I} \Sigma_{2}^{0}$, we have $P(n)$. (Note that closure under sequences of variable substitutions also holds, by Remark 5.4.) The proof that (1) implies (4) is similar.

That (2), (3), (4), and (5) all imply (1) follows from Theorems 6.11 and 6.12 below (using condition (a) in item (3) of Theorem 6.11), together with the equivalence between $\mathrm{I} \Sigma_{2}^{0}$ and the finite $\Pi_{1}^{0}$-recursion principle.

Belanger [3] has shown that, for listings of all types of a theory with countably many types, the situation is different

THEOREM 5.12 (Belanger [3]). The following statements are equivalent over $\mathrm{RCA}_{0}$.

(1) The disjunction $\mathrm{WKL}_{0} \vee \mathrm{I} \Sigma_{2}^{0}$.

(2) If a list of all the types of a theory satisfies the pairwise full amalgamation closure conditions then it satisfies the finite full amalgamation closure conditions.

(3) If a list of all the types of a theory satisfies the pairwise free amalgamation closure conditions then it satisfies the finite free amalgamation closure conditions.

(4) If a list of all the types of a theory satisfies the pairwise full amalgamation closure conditions then it satisfies the finite free amalgamation closure conditions.

\subsection{Spectrum enumeration existence theorems}

Theorems 5.1 and 5.7 are "if and only if" statements. The "if" directions are the main focus of this paper. In this section, we analyze the reverse mathematics of the "only if" directions. These are easily seen to hold in $\mathrm{RCA}_{0}$, though in the case of Theorem 5.1, we have to choose the appropriate notion of homogeneity.

Proposition 5.13. The following is provable in $\mathrm{RCA}_{0}$. Let $\mathcal{A}$ be a model. Then $\mathbb{T}(\mathcal{A})$ has an enumeration $X$ satisfying the finite free amalgamation closure conditions. If $\mathcal{A}$ is 1-point homogeneous then $X$ can be chosen to satisfy the 1-point full amalgamation closure conditions. If $\mathcal{A}$ is 1-homogeneous then $X$ can be chosen to satisfy the pairwise full amalgamation closure conditions. If $\mathcal{A}$ is homogeneous then $X$ can be chosen to satisfy the finite full amalgamation closure conditions. 
Proof. Let $\mathcal{A}$ be a model, let $\bar{c}_{0}, \bar{c}_{1}, \ldots$ be an enumeration of the $A$-tuples, and for each $i$, let $\bar{x}_{i, 0}, \bar{x}_{i, 1}, \ldots$ be an enumeration of the tuples of variables of the same length as $\bar{c}_{i}$ (chosen so that if $\left|\bar{c}_{i}\right|=\left|\bar{c}_{i^{\prime}}\right|$ then $\bar{x}_{i, j}=\bar{x}_{i^{\prime}, j}$ for all $j$ ). Let $X=\left\{q_{i}\left(\bar{x}_{i, j}\right)\right\}_{i, j \in \mathbb{N}}$, where $q_{i}$ is the type of $\bar{c}_{i}$ in the variables $\bar{x}_{i, j}$. Then $X$ is an enumeration of $\mathbb{T}(\mathcal{A})$. It is easy to see that $X$ satisfies conditions (1)-(4) in Theorem 5.1 and is closed under sequences of variable substitutions.

Let $q_{i_{0}}\left(\bar{x}_{i_{0}, j_{0}}\right), \ldots, q_{i_{n}}\left(\bar{x}_{i_{n}, j_{n}}\right)$ be elements of $X$ whose free variables do not overlap. Let $i$ be such that $\bar{c}_{i}$ is the tuple $\bar{c}_{i_{0}} \ldots \bar{c}_{i_{n}}$, and let $j$ be such that $\bar{x}_{i, j}$ is the tuple $\bar{x}_{i_{0}, j_{0}} \ldots \bar{x}_{i_{n}, j_{n}}$. Then $q_{i_{0}}\left(\bar{x}_{i_{0}, j_{0}}\right), \ldots, q_{i_{n}}\left(\bar{x}_{i_{n}, j_{n}}\right) \subseteq q_{i}\left(\bar{x}_{i, j}\right)$. Thus $X$ satisfies the finite free type amalgamation condition.

Now suppose that $\mathcal{A}$ is homogeneous and let $q_{i_{0}}\left(\bar{x}_{i_{0}, j_{0}}\right), \ldots, q_{i_{n}}\left(\bar{x}_{i_{n}, j_{n}}\right)$ be a consistent sequence of elements of $X$. By the definition of consistency, we may assume that all the free variables shared by two or more of these types are in $\bar{x}_{i_{0}, j_{0}}$, so we can write each $q_{i_{k}}\left(\bar{x}_{i_{k}, j_{k}}\right)$ as $q_{i_{k}}\left(\bar{y}_{i_{k}} \bar{z}_{i_{k}}\right)$, where $\bar{y}_{i_{k}} \subseteq \bar{x}_{i_{0}, j_{0}}$ and the $\bar{z}_{i_{k}}$ are disjoint from $\bar{x}_{i_{0}, j_{0}}$ and pairwise disjoint. The types $q_{i_{k}}\left(\bar{y}_{i_{k}} \bar{z}_{i_{k}}\right) \uparrow \bar{y}_{i_{k}}$ are all compatible, so homogeneity implies that for each $k \leqslant n$, there is an $i_{k}^{\prime}$ such that $q_{i_{k}^{\prime}}\left(\bar{x}_{i_{k}^{\prime}, j_{k}}\right)=q_{i_{k}}\left(\bar{x}_{i_{k}, j_{k}}\right)$ and if the $p$ th element of $\bar{x}_{i_{0}, j_{0}}$ equals the $q$ th element of $\bar{x}_{i_{k}^{\prime}, j_{k}}$, then the $p$ th element of $\bar{c}_{i_{0}}$ equals the $q$ th element of $\bar{c}_{i_{k}^{\prime}}$. For each $k \leqslant n$, let $\bar{d}_{k}$ be the elements of $\bar{c}_{i_{k}}$ corresponding to the variables in $\bar{z}_{i_{k}}$. Let $i$ be such that $\bar{c}_{i}$ is the tuple $\bar{c}_{i_{0}} \bar{d}_{i_{1}} \ldots \bar{d}_{i_{n}}$. Let $j$ be such that $\bar{x}_{i, j}$ is the tuple $\bar{x}_{i_{0}, j_{0}} \bar{z}_{i_{1}, j_{1}} \ldots \bar{z}_{i_{n}, j_{n}}$. Then $q_{i_{0}}\left(\bar{x}_{i_{0}, j_{0}}\right), \ldots, q_{i_{n}}\left(\bar{x}_{i_{n}, j_{n}}\right) \subseteq q_{i}\left(\bar{x}_{i, j}\right)$. Thus $X$ satisfies the finite full type amalgamation condition.

A similar argument shows that if $\mathcal{A}$ is 1-point homogeneous then $X$ satisfies the 1-point full type amalgamation condition, while if $\mathcal{A}$ is 1-homogeneous then $X$ satisfies the pairwise full type amalgamation condition.

The proof of Theorem 4.3 can easily be modified to obtain the following result.

THEOREM 5.14. The following are equivalent over $\mathrm{RCA}_{0}$.

(1) $\mathrm{I} \Sigma_{2}^{0}$

(2) For every 1-point homogeneous model $\mathcal{A}$, there is an enumeration of $\mathbb{T}(\mathcal{A})$ satisfying the pairwise full amalgamation closure conditions.

(3) For every 1-point homogeneous model $\mathcal{A}$, there is an enumeration of $\mathbb{T}(\mathcal{A})$ satisfying the finite full amalgamation closure conditions.

Proof. That (1) implies (2) and (3) follows from Theorem 5.11 and Proposition 5.13. Clearly (3) implies (2). To see that (2) implies (1), let $\varphi, n, z, \theta$, and $\mathcal{M}$ be as in the proof of Theorem 4.3 and suppose that $\mathbb{T}(\mathcal{M})$ has an enumeration $X$ satisfying the pairwise full amalgamation closure conditions. Let $p\left(x, y, z_{0}, \ldots, z_{n-1}\right)$ be the type of

$$
(\langle 0,0,0\rangle,\langle 1,0,0\rangle,\langle 0,1,0\rangle, \ldots,\langle 0,1, n-1\rangle)
$$

in $\mathcal{M}$, and let $q\left(y, w_{0}, \ldots, w_{n-1}\right)$ be the type of

$$
(\langle 0,0,0\rangle,\langle 0,1,0\rangle, \ldots,\langle 0,1, n-1\rangle)
$$

in $\mathcal{M}$. Then $p\lceil y=q \uparrow y$, so there is a type

$$
r\left(x, y, z_{0}, \ldots, z_{n-1}, w_{0}, \ldots, w_{n-1}\right)
$$

in $X$ amalgamating these two types. Let $\left(a, b, c_{0}, \ldots, c_{n-1}, d_{0}, \ldots, d_{n-1}\right)$ realize this type in $\mathcal{M}$. Then either each $c_{i}$ is of the form $\langle 1,1, k\rangle$ where $\theta(k)$ holds, or 
each $d_{i}$ is of this form. In either case, as in the proof of Theorem 4.3, the instance of $\Pi_{1}^{0}$-recursion corresponding to $\varphi, n$, and $z$ holds.

Similarly, the proof of Theorem 4.4 yields the following result.

THEOREM 5.15. The following are equivalent over $\mathrm{RCA}_{0}$.

(1) $\mathrm{B} \Sigma_{2}^{0}$

(2) For every 1 -homogeneous model $\mathcal{A}$, there is an enumeration of $\mathbb{T}(\mathcal{A})$ satisfying the finite full amalgamation closure conditions.

(3) For every strongly 1-homogeneous model $\mathcal{A}$, there is an enumeration of $\mathbb{T}(\mathcal{A})$ satisfying the finite full amalgamation closure conditions.

Proof. That (1) implies (2), and hence (3), follows from Theorem 4.4 and Proposition 5.13. To see that (3) implies (1), and hence so does (2), let $\Psi$, $n$, and $\mathcal{M}$ be as in the proof of Theorem 4.4, and suppose that $\mathbb{T}(\mathcal{M})$ has an enumeration $X$ satisfying the finite full amalgamation closure conditions. Let $p\left(x_{0}, \ldots, x_{n-1}, y_{0}, \ldots, y_{n-1}\right)$ be the type of

$$
(\langle 0,0,0,0\rangle, \ldots,\langle n-1,0,0,0\rangle,\langle 0,1,0,0\rangle, \ldots,\langle n-1,1,0,0\rangle)
$$

in $\mathcal{M}$. For $i<n$, let $q_{i}\left(x_{i}, z_{i}\right)$ be the type of $(\langle i, 0,0,0\rangle,\langle i, 0,0,1\rangle)$ in $\mathcal{M}$. The sequence $q_{0}, \ldots, q_{n-1}$ exists as a sequence of elements of $X$ by closure under sequences of variable substitutions, since $X$ contains the type of

$$
(\langle 0,0,0,0\rangle,\langle 0,0,0,1\rangle, \ldots,\langle n-1,0,0,0\rangle,\langle n-1,0,0,1\rangle) .
$$

For the same reason, $X$ contains the sequence of types $q_{i}\left[y_{i}, w_{i}\right]$ (using the notation introduced in Remark 5.9), where the $w_{i}$ are pairwise distinct from the $z_{i}$. Then there is a type

$$
r\left(x_{0}, \ldots, x_{n-1}, y_{0}, \ldots, y_{n-1}, z_{0}, \ldots, z_{n-1}, w_{0}, \ldots, w_{n-1}\right)
$$

in $X$ that amalgamates $p\left(x_{0}, \ldots, x_{n-1}, y_{0}, \ldots, y_{n-1}\right)$ with $q_{i}\left(x_{i}, z_{i}\right)$ and $q_{i}\left[y_{i}, w_{i}\right]$ for all $i<n$. This type must be realized in $\mathcal{M}$ by some tuple

$$
\left(a_{0}, \ldots, a_{n-1}, b_{0}, \ldots, b_{n-1}, c_{0}, \ldots, c_{n-1}, d_{0}, \ldots, d_{n-1}\right) .
$$

For each $i<n$, exactly one of $c_{i}$ or $d_{i}$ is of the form $\left\langle i, 1, l, 2\left\langle m_{i}, k_{i}\right\rangle+1\right\rangle$, in which case we have $\left(\exists u<m_{i}\right) \Psi(i, u)$. Thus we obtain a sequence $m_{0}, \ldots, m_{n-1}$ whose maximum witnesses the satisfaction of the instance of $\mathrm{B}_{1}^{0}$ in the proof of Theorem 4.4 .

We finish this section with the following result, mentioned above following Proposition 5.10.

THEOREM 5.16. The following are equivalent over $\mathrm{RCA}_{0}$.

(1) $\mathrm{B} \Sigma_{2}^{0}$

(2) If the lists of types $X$ and $Y$ are equal as sets of types and $X$ satisfies the finite (free or full) type amalgamation condition, then so does $Y$.

(3) Every enumeration of the type spectrum of a model satisfies the finite free type amalgamation condition.

(4) Every enumeration of the type spectrum of a homogeneous model satisfies the finite full type amalgamation condition. 
Proof. By Proposition 5.13, (3) and (4) follow from (2) over RCA $\mathrm{A}_{0}$. We show that (2) holds in $\mathrm{RCA}_{0}+\mathrm{B} \Sigma_{2}^{0}$. We do the argument for full amalgamation, as the argument for free amalgamation is essentially the same. Let $X$ and $Y$ be lists of types that are equal as sets, and suppose that $X$ satisfies the finite full type amalgamation condition. Let $q_{0}, \ldots, q_{k} \in Y$ be a consistent sequence of types. The property of an element of $X$ being equal to an element of $Y$ is $\Pi_{1}^{0}$, and for each $i \leqslant k$ there is an element of $X$ equal to $q_{i}$, so by the finite axiom of choice for $\Pi_{1}^{0}$ properties, there is a sequence $p_{0}, \ldots, p_{k} \in X$ such that $p_{i}=q_{i}$ for all $i \leqslant k$. Then there is a $p \in X$ amalgamating $p_{0}, \ldots, p_{k}$. There is a $q \in Y$ such that $q=p$, and this $q$ amalgamates $q_{0}, \ldots, q_{k}$.

For the other direction, we assume that at least one of (2), (3), or (4) holds and argue in $\mathrm{RCA}_{0}$. Let $Q$ be a $\Pi_{1}^{0}$ property and $m$ be such that $(\forall i<m)(\exists u) Q(i, u)$. Let $R$ be a $\Delta_{1}^{0}$ property such that $Q(x, y)$ if and only if $(\forall z) R(x, y, z)$. Let $\mathcal{T}=2^{<\mathbb{N}}$ and let $T=T(\mathcal{T})$ be as in Definition 3.5. We define a structure $\mathcal{M}$ in the language of $T$ with domain $\mathbb{N}$. For $n \in \mathbb{N}$ and $\alpha \in 2^{\mathbb{N}}$, we write $\mathcal{M} \vDash P^{\alpha}(n)$ to mean that $\mathcal{M} \vDash P_{k}(n)$ if and only if $\alpha(k)=1$. Let $A_{\sigma}$ for $\sigma \in 2^{<\mathbb{N}}$ and $B_{i, j}$ for $i<m$ and $j \in \mathbb{N}$ be pairwise disjoint infinite sets whose union is $\mathbb{N}$. For each $\sigma \in 2^{<\mathbb{N}}$, let $\mathcal{M} \vDash P^{\sigma 0^{\mathbb{N}}}(n)$ for all $n \in A_{\sigma}$. For $i<m$ and $j \in \mathbb{N}$, let $\alpha_{i, j}(k)$ be 1 if $(\forall z<k) R(i, j, z)$ and 0 otherwise. Let $\mathcal{M} \vDash P^{0^{i} 1 \alpha_{i, j}}(n)$ for all $n \in B_{i, j}$.

It is easy to check that $\mathcal{M}$ is a homogeneous model of $T$, so by Proposition 5.13, $\mathbb{T}(\mathcal{M})$ has an enumeration $\left\{p_{i}\right\}_{i \in \mathbb{N}}$ satisfying the finite full amalgamation closure conditions. By effective quantifier elimination, to define a 1-type $p(x)$ of $T$, it is enough to specify which $P_{k}(x)$ are in $p$. For $i<m$, let $q_{i}\left(x_{i}\right)$ be the 1-type containing $P_{k}\left(x_{i}\right)$ iff $k \geqslant i$ (i.e., the type corresponding to $P^{0^{i} 1^{\mathbb{N}}}\left(x_{i}\right)$ ), where the $x_{i}$ are pairwise distinct variables. For $k \in \mathbb{N}$, let $q_{m+k}=p_{k}$. It is easy to check that $Y=\left\{q_{i}\right\}_{i \in \mathbb{N}}$ is also an enumeration of $\mathbb{T}(\mathcal{M})$. Since we are assuming that at least one of (2), (3), or (4) holds, $Y$ satisfies the finite free amalgamation closure conditions. Then there is a type in $Y$ amalgamating $q_{0}, \ldots, q_{m-1}$. This type is realized in $\mathcal{M}$, so there is a sequence $n_{0}, \ldots, n_{m-1} \in \mathbb{N}$ such that $\mathcal{M} \vDash P^{0^{i} 1^{\mathbb{N}}}\left(n_{i}\right)$ for all $i<m$. Then $n_{i} \in B_{i, j_{i}}$ for some $j_{i}$ such that $(\forall z) R\left(i, j_{i}, z\right)$, and we can form the sequence $j_{0}, \ldots, j_{m-1}$. Letting $b$ be greater than all the $j_{i}$, we have $(\forall i<m)(\exists u<b) Q(i, u)$.

Note that $\mathrm{B} \Sigma_{2}^{0}$ is also enough to show that closure under sequences of variable substitutions is enumeration-independent (by an argument similar to that in the first part of the above proof), so the above theorem remains true if we replace "satisfies the finite (free or full) type amalgamation condition" by "satisfies the finite (free or full) amalgamation closure conditions".

\subsection{Model existence theorems}

As discussed in the introduction, the degree-theoretic results on atomic and homogeneous models obtained by Csima [7] and Csima, Hirschfeldt, Knight, and Soare $[\mathbf{8}]$, on the one hand, and Lange $[\mathbf{2 7}, \mathbf{2 8}]$, on the other, are almost identical. Thus it is natural to compare AMT with analogous statements for homogeneous models. The following are three reasonable candidates, which match our sets of full amalgamation closure conditions with the corresponding notions of homogeneity. Of course, when formalizing the statements below, a set of types $X$ is given as a 
list of types $\left\{p_{i}\right\}_{i \in \mathbb{N}}$, and $\mathbb{T}(\mathcal{A})=X$ means that $X$ is an enumeration of the type spectrum of $\mathcal{A}$ (i.e., a type is realized in $\mathcal{A}$ iff it is equal to $p_{i}$ for some $i$ ).

HMT (Homogeneous Model Theorem): Let $T$ be a theory and let $X \subseteq$ $S(T)$ satisfy the finite full amalgamation closure conditions. Then there exists a homogeneous model $\mathcal{A}$ of $T$ with $\mathbb{T}(\mathcal{A})=X$.

1-HMT (1-Homogeneous Model Theorem): Let $T$ be a theory and let $X \subseteq$ $S(T)$ satisfy the pairwise full amalgamation closure conditions. Then there exists a 1-homogeneous model $\mathcal{A}$ of $T$ with $\mathbb{T}(\mathcal{A})=X$.

1-PHMT (1-Point Homogeneous Model Theorem): Let $T$ be a theory and let $X \subseteq S(T)$ satisfy the 1-point full amalgamation closure conditions. Then there exists a 1-point homogeneous model $\mathcal{A}$ of $T$ with $\mathbb{T}(\mathcal{A})=X$.

We also name the following model existence theorems.

SMT (Strong Model Existence Theorem): Let $T$ be a theory and let $X \subseteq$ $S(T)$ satisfy the pairwise free amalgamation closure conditions. Then there exists a model $\mathcal{A}$ of $T$ with $\mathbb{T}(\mathcal{A})=X$.

WMT (Weak Model Existence Theorem): Let $T$ be a theory and let $X \subseteq$ $S(T)$ satisfy the finite free amalgamation closure conditions. Then there exists a model $\mathcal{A}$ of $T$ with $\mathbb{T}(\mathcal{A})=X$.

To discuss the reverse mathematical strength of these theorems, we first need to examine the effective versions of Theorems 5.1 and 5.7, which requires introducing some strengthenings of the extension condition in these theorems. 



\section{CHAPTER 6}

\section{Extension Functions and Model Existence}

\subsection{Extension functions}

Theorem 5.1 gives closure conditions that a set of types must satisfy to be the type spectrum of a homogeneous model. However, these conditions do not tell us how to build such a model effectively from a listing of such a set. The following result answered a question of Morley [36].

Theorem 6.1 (Goncharov [15], Peretyat'kin [41], and Millar [31]). There is a computable list of types $X$ satisfying all the conditions in Theorem 5.1 such that $X$ is not an enumeration of the type spectrum of any decidable homogeneous model.

Goncharov [15] and Peretyat'kin [41] realized that in order to build a decidable homogeneous model $\mathcal{M}$ such that $X$ is an enumeration of $\mathbb{T}(\mathcal{M})$, we need to effectivize one or more of the conditions in Theorem 5.1, to obtain some computable information about how the types in the computable list $X$ extend one another. Of particular interest are the extension condition and the (pairwise full) type amalgamation condition. Since we naturally seek the weakest possible hypothesis guaranteeing the existence of a decidable homogeneous model $\mathcal{M}$ such that $X$ is an enumeration of the type spectrum of $\mathcal{M}$, a reasonable way to proceed is to begin by considering a decidable homogeneous model $\mathcal{M}$ and the natural listing $X=\left\{q_{i}\left(\bar{x}_{i, j}\right)\right\}_{i, j \in \mathbb{N}}$ of its type spectrum given in the proof of Theorem 5.13, and determining which of these conditions hold effectively of $X$.

Let us recall the definition of $X$ for convenience: Let $\bar{c}_{0}, \bar{c}_{1}, \ldots$ be an enumeration of the $M$-tuples, and for each $i$, let $\bar{x}_{i, 0}, \bar{x}_{i, 1}, \ldots$ be an enumeration of the tuples of variables of the same length as $\bar{c}_{i}$. Let $X=\left\{q_{i}\left(\bar{x}_{i, j}\right)\right\}_{i, j \in \mathbb{N}}$, where $q_{i}$ is the type of $\bar{c}_{i}$ in the variables $\bar{x}_{i, j}$.

Let $p_{\langle i, j\rangle}=q_{i}\left(\bar{x}_{i, j}\right)$. The effective version of pairwise full type amalgamation says that there is a computable function $f$ such that, if $p_{m}$ and $p_{n}$ are basically consistent, then $p_{m} \cup p_{n} \subseteq p_{f(m, n)}$. We claim this effective closure condition does not necessarily hold. To see that this is the case, let $\mathcal{M}$ be as in the proof of Proposition 4.2. We adopt the notation of that proof.

Let $a, b \in M$ be such that $a \equiv b$ and $\neg E^{\mathcal{M}}(a, b)$. Now assume for a contradiction that there is a computable function $f$ as above. We show how to compute $\emptyset^{\prime}$ using $f$. Given $i$, let $c, d \in M$ be such that $\mathcal{M} \vDash R_{i}(c) \wedge R_{i}(d) \wedge E(a, c) \wedge E(b, d)$. Let $y_{0}, y_{1}, y_{2}$ be variables, let $m$ be such that $p_{m}$ is the type of $a c$ in $y_{0}, y_{1}$, and let $n$ be such that $p_{n}$ is the type of $b d$ in $y_{0}, y_{2}$. Then $p_{m}$ and $p_{n}$ are basically consistent, so $p_{m} \cup p_{n} \subseteq p_{f(m, n)}$. Let $i$ and $j$ be such that $f(m, n)=\langle i, j\rangle$, and let $e_{0}, e_{1}, e_{2} \in M$ be the elements of $\bar{c}_{i}$ corresponding to the positions of $y_{0}, y_{1}, y_{2}$ in $\bar{x}_{i, j}$, respectively. Then $e_{1} \equiv c$ and $e_{2} \equiv d$. Furthermore, $E^{\mathcal{M}}$ holds of $\left(e_{0}, e_{1}\right)$ and of $\left(e_{0}, e_{2}\right)$, and hence of $\left(e_{1}, e_{2}\right)$, so either $\neg E^{\mathcal{M}}\left(e_{1}, c\right)$ or $\neg E^{\mathcal{M}}\left(e_{2}, d\right)$. We assume that $\neg E^{\mathcal{M}}\left(e_{1}, c\right)$, as the other case is essentially the same. We also assume that $e_{1}$ is even, and hence 
on the $\mathcal{A}$ side of $\mathcal{M}$, and $c$ is odd, and hence on the $\mathcal{B}$ side of $\mathcal{M}$. Again, the other case is essentially the same.

If $i \in \emptyset_{\max \left(e_{1}, c\right)}^{\prime}$ then of course $i \in \emptyset^{\prime}$. Otherwise, we claim that $i \notin \emptyset^{\prime}$. Suppose this is not the case. Then $i$ enters $\emptyset^{\prime}$ at a stage $t>\max \left(e_{1}, c\right)$. By our construction of $\mathcal{M}$, we have $\mathcal{M} \vDash R_{i, t}\left(e_{1}\right)$ and $\mathcal{M} \vDash \neg R_{i, t}(c)$, which contradicts the fact that $e_{1} \equiv c$.

Thus $\mathcal{M}$ provides us an example of a decidable homogeneous model such that the natural listing of $\mathbb{T}(\mathcal{M})$ does not satisfy the effective version of the pairwise full type amalgamation condition. For the extension condition, however, the situation is different. Let us return to general case of a decidable homogeneous model $\mathcal{M}$ and the listing $X=\left\{q_{i}\left(\bar{x}_{i, j}\right)\right\}_{i, j \in \mathbb{N}}$ of $\mathbb{T}(\mathcal{M})$. Again let $p_{\langle i, j\rangle}=q_{i}\left(\bar{x}_{i, j}\right)$, and let $\theta_{0}, \theta_{1}, \ldots$ list the formulas in our language. The effective version of extension says that there is a computable function $f$ such that if $\theta_{n}(\bar{y} \bar{z})$ is consistent with $p_{m}(\bar{y})$, then $p_{f(m, n)}$ extends both $p_{m}$ and $\theta_{n}$. This condition does in fact hold of $X$ : Given such $m$ and $n$, we simply let $i$ and $j$ be such that $m=\langle i, j\rangle$ and search for $\bar{c}_{k}$ extending $\bar{c}_{i}$ such that $\mathcal{M} \vDash \theta_{n}\left(\bar{c}_{k}\right)$. We then let $l$ be such that $\bar{x}_{k, l}=\bar{y} \bar{z}$, and let $f(m, n)=\langle k, l\rangle$. Note that this argument does not use the assumption that $\mathcal{M}$ is homogeneous.

Goncharov [15] and Peretyat'kin [41] showed that the existence of such a computable extension function is in fact a sufficient condition on a computable list of types $X$ satisfying the pairwise full amalgamation closure conditions to ensure that there is a decidable homogeneous model $\mathcal{M}$ such that $X$ is an enumeration of the type spectrum of $\mathcal{M}$. Indeed, they went even further, by showing that we do not need to be able to compute an extension function, but merely computably approximate one. As we will see in Theorem 6.4, this weaker condition is not only sufficient, but also necessary. The following definitions make the above notions precise. For a detailed look at the following definitions and their uses in the context of atomic and homogeneous models, see Lange and Soare [29].

Definition 6.2. Let $X=\left\{p_{i}\right\}_{i \in \mathbb{N}}$ be a list of types of a theory $T$ and let $\theta_{0}, \theta_{1}, \ldots$ be an effective enumeration of the formulas in the language of $T$.

(i) A function $f(i, j)$ is an extension function for $X$ if for every $n$-type $p_{i}(\bar{x})$ and $(n+k)$-ary $\theta_{j}(\bar{x} \bar{y})$ consistent with $p_{i}(\bar{x})$, the $(n+k)$-type $p_{f(i, j)}$ extends both $p_{i}(\bar{x})$ and $\theta_{j}(\bar{x} \bar{y})$, i.e.,

$$
p_{i}(\bar{x}) \cup\left\{\theta_{j}(\bar{x} \bar{y})\right\} \subseteq p_{f(i, j)}(\bar{x} \bar{y}) .
$$

(ii) A function $g(i, j, s)$ is an extension function approximation if for every $n$ type $p_{i}(\bar{x})$ and $(n+k)$-ary $\theta_{j}(\bar{x} \bar{y})$ consistent with $p_{i}(\bar{x})$,

a. $\lim _{s} g(i, j, s)$ exists,

b. $p_{i}(\bar{x}) \cup\left\{\theta_{j}(\bar{x} \bar{y})\right\} \subseteq p_{\lim _{s} g(i, j, s)}(\bar{x} \bar{y})$, and

c. $p_{g(i, j, s)} \uparrow s=p_{g(i, j, s+1)}\lceil s$ for all $s$.

Thus, an extension function is one that, given an index for an $n$-type and an $(n+k)$-ary formula consistent with that type, returns an index for a type amalgamating the type and the formula. An extension function approximation, given the same data, approximates the index of such an amalgamating type, in such a way that the stage $s$ guess at a true amalgamator agrees with the true amalgamator on the first $s$ many formulas. (The last condition has led to this notion being called "monotone extension function approximation", but since this is 
the only kind of extension function approximation we consider, we omit the word "monotone".)

THEOREM 6.3 (Goncharov [15] (for homogeneous models) and Peretyat'kin [41] (for both cases)). Let $X$ be a computable list of types of a decidable theory $T$ such that $X$ satisfies the pairwise free amalgamation closure conditions and has a computable extension function approximation. Then there is a decidable model $\mathcal{A}$ such that $X$ is an enumeration of $\mathbb{T}(\mathcal{A})$. If $X$ also satisfies the pairwise full amalgamation closure conditions, then $\mathcal{A}$ can be chosen to be homogeneous.

The basic idea of the proof of this result is to perform a Henkin construction of a model $\mathcal{A}$ of $T$ while ensuring that two families of requirements are met: ones stating that each type in $X$ is realized by some $A$-tuple, and ones stating that each $A$-tuple realizes a type in $X$. To satisfy the first family of requirements, we assign types in $X$ to $A$-tuples. These assignments might change in the course of the construction, but to satisfy the second family of requirements, we ensure that for each $A$-tuple $\bar{a}$, there is a type $p \in X$ such that we eventually permanently assign $p$ to $\bar{a}$. Since we do not have effective type amalgamation, our type assignments may turn out to be in conflict. To deal with this issue, we use a priority construction: We might assign types $p, q \in X$ to the tuples $\bar{a} \bar{b}$ and $\bar{a} \bar{c}$, respectively, and find out at some later point in the construction that $p \uparrow \bar{a} \neq q \uparrow \bar{a}$. In this case, we maintain whichever assignment has been made by the stronger priority requirement, say the assignment of $p$ to $\bar{a} \bar{b}$, and reassign $q$ to some new tuple of elements. We now have the problem of assigning some new type to $\bar{a} \bar{c}$. We do so by using our computable extension function approximation to guess at a type $r$ in $X$ extending both $p$ and the formula representing the information we have already added to the elementary diagram of $\mathcal{A}$ about the tuple $\bar{a} \bar{b} \bar{c}$, and assigning $r \uparrow \bar{a} \bar{c}$ to $\bar{a} \bar{c}$. Of course, our extension function approximation can change its mind on what $r$ is, but only finitely often. Thus we have a finite injury construction.

The existence of a computable extension function is enumeration-dependent, in the sense that the type spectrum of a model $\mathcal{A}$ can have computable enumerations $X$ and $Y$ such that $X$ has a computable extension function but $Y$ does not. Extension function approximations are more robust: if some computable enumeration of $\mathbb{T}(\mathcal{A})$ has a computable extension function approximation, then every computable enumeration of $\mathbb{T}(\mathcal{A})$ has one. (See Lange and Soare [29, Section 4].) We give the reverse mathematical version of the latter fact in part (2) of Proposition 6.6.

The following result shows how extension functions can be used to characterize the computable lists of types that are enumerations of type spectra of decidable (homogeneous) models. It also shows that Theorem 6.3 is tight, in the sense that having a computable extension function approximation is in fact a necessary condition for a list of types be an enumeration of the type spectrum of some decidable model.

Theorem 6.4 (Goncharov [15] (for homogeneous models) and Peretyat'kin [41] (for both cases)). Let $T$ be a decidable theory and $\mathcal{A}$ a (homogeneous) model of $T$ such that $\mathbb{T}(\mathcal{A})$ has a computable enumeration. Then the following are equivalent.

(1) $\mathcal{A}$ has a decidable copy.

(2) Every computable enumeration of $\mathbb{T}(\mathcal{A})$ has a computable extension function approximation. 
(3) Some computable enumeration of $\mathbb{T}(\mathcal{A})$ has a computable extension function approximation.

(4) Some computable enumeration of $\mathbb{T}(\mathcal{A})$ has a computable extension function.

In computable model theory, it is common to weaken the hypotheses of this definition by considering only the case $k=1$, i.e., only formulas of the form $\theta_{j}(\bar{x} y)$. Let us call the concepts defined in this way weak extension function and weak extension function approximation. Computability theoretically, there is no real difference: it is not difficult to show that if $X$ has a computable weak extension function then it has a computable extension function, and if it has a computable weak extension function approximation, then it has a computable extension function approximation. In $\mathrm{RCA}_{0}$, it is still easy show that the existence of a weak extension function implies that of an extension function, but the situation is less clear in the case of extension function approximations. The issue is the usual one that we cannot always show in $\mathrm{RCA}_{0}$ that the composition of finitely many functions with limits has a limit.

The only place below where the difference between weak extension function approximations and extension function approximations matters is in the proof of Theorem 6.10. In all other cases, when we use the existence of an extension function approximation, we actually use only the existence of a weak extension function approximation.

The principle that if $X$ has a weak extension function approximation then it has an extension function approximation is easily provable from $\mathrm{RCA}_{0}+\mathrm{I}_{2}^{0}$, and part (2) of Theorem 7.1 below implies that it also follows from ATT (assuming $X$ in fact satisfies the extension condition), but we do not what else can be said about its strength.

Classically, the definition of extension function approximation looks a bit awkward, and it would seem better to define it as an approximation to an extension function with the additional property (c). However, in the reverse mathematical setting, the existence of an extension function approximation $g$ does not guarantee the existence of the extension function $f(i, j)=\lim _{s} g(i, j, s)$. Indeed, while every extension function can easily be transformed into an extension function approximation of itself, there are examples of computable lists of types that have computable extension function approximations but no computable extension functions. In fact, we have the following equivalence.

Proposition 6.5. The statement that every list of types of a theory with an extension function approximation has an extension function is equivalent to $\mathrm{ACA}_{0}$ over $\mathrm{RCA}_{0}$. This fact remains true if we restrict attention to lists of types satisfying any one of the sets of closure conditions discussed above.

Proof. $\mathrm{ACA}_{0}$ is clearly enough to show that the limit of an extension function approximation exists and is an extension function. For the reversal, fix a function $f$. We argue in $\mathrm{RCA}_{0}$ and build a list of types $X$ of a theory $T$ such that $X$ satisfies the finite full amalgamation closure conditions and has an extension function approximation, so that if $X$ has an extension function then the range of $f$ exists as a set.

We work in the language with unary relation symbols $P_{i}$ for $i \in \mathbb{N}$. Let $A$ be the following set of axioms.

(1) $P_{i+1}(x) \rightarrow P_{i}(x)$ for all $i \in \mathbb{N}$. 
(2) $\left(\exists^{>k} x\right)\left[P_{i}(x) \wedge \neg P_{i+1}(x)\right]$ for all $i, k \in \mathbb{N}$.

(3) $\left(\exists^{>k} x\right) \neg P_{0}(x)$ for all $k \in \mathbb{N}$.

These axioms are the same as in the proof of Theorem 4.3, except that here we do not need the symbol $E$, so we can show that $A$ admits effective quantifier elimination and that its deductive closure $T$ is a theory using a simpler version of the argument in the proof of that theorem.

By effective quantifier elimination, the types of $T$ can be identified with atomic types. Thus the 1-types of $T$ in a given variable $x$ are the principal types $r_{i}(x)$ generated by $P_{i-1}(x) \wedge \neg P_{i}(x)$ (where the first conjunct is omitted if $i=0$ ), and the nonprincipal type $r_{\infty}(x)$, which has $P_{i}(x)$ for all $i \in \mathbb{N}$.

We now give names for all the types of $T$. Let $E$ be an equivalence relation on some $n \in \mathbb{N}$, let $\sigma=\left(k_{0}, \ldots, k_{n-1}\right)$ be such that $k_{i} \in \mathbb{N} \cup\{\infty\}$ for $i<n$ and $k_{i}=k_{j}$ whenever $E(i, j)$ holds (note that $E$ determines $n$ uniquely), and let $\left(y_{0}, \ldots, y_{n-1}\right)$ be a tuple of variables. Let $s_{E, \sigma}\left(y_{0}, \ldots, y_{n-1}\right)$ be the $n$-type that includes $y_{i}=y_{j}$ if $E(i, j)$ holds and $y_{i} \neq y_{j}$ if $\neg E(i, j)$ holds, and states that each $y_{i}$ has type $r_{k_{i}}$. Note that these conditions completely specify an $n$-type, since we have only unary relation symbols. Furthermore, every type of $T$ is equal to $s_{E, \sigma}(\bar{y})$ for some equivalence relation $E$, some compatible $\sigma$, and some tuple of variables $\bar{y}$.

Let $x_{0}, x_{1}, \ldots$ be the variables in our language. Clearly, we can enumerate all the types $s_{E, \sigma}\left(x_{i_{0}}, \ldots, x_{i_{n-1}}\right)$, where $E$ and $\sigma$ are as above and $i_{0}, \ldots, i_{n-1}$ are distinct numbers, as a list $q_{0}, q_{1}, \ldots$ with an extension function $h$ (relative to a fixed listing $\theta_{0}, \theta_{1}, \ldots$ of the formulas in our language) such that if $q_{i}$ is a type in the variables $\bar{x}$ and the free variables of $\theta_{j}$ are $\bar{y}$ then $q_{h(i, j)}$ is a type in the variables $\bar{x} \cup \bar{y}$. We may further assume that $h$ is defined so that, for $i$ and $j$ as above, if $x_{k} \in \bar{y} \backslash \bar{x}$ then $q_{h(i, j)} \backslash x_{k}=r_{l}\left(x_{k}\right)$ for some $l \in \mathbb{N}$. We also assume our types are listed so that for any type $p$ and any $m \in \mathbb{N}$, the restriction $p\lceil m$ includes no formulas involving $P_{m}$.

We now define $X=\left\{p_{i}\right\}_{i \in \mathbb{N}}$, which in fact includes all types of $T$. Fix $k, c \in \mathbb{N}$. In defining $p_{\langle k, c\rangle}$, there are two cases. If $q_{k}=s_{E,\left(\infty, l_{1}, \ldots, l_{n+1}\right)}\left(x_{0}, \ldots, x_{n+1}\right)$, where $\neg E(0,1)$ and $l_{1} \in \mathbb{N}$ is such that $(\forall m<c) \neg f(m)=l_{1}$, then let $p_{\langle k, c\rangle}\left(x_{0}, \ldots, x_{n+1}\right)$ be the type determined by stating that $x_{0} \neq x_{1}$, that $x_{j}$ has type $r_{l_{j}}$ for $j \in[1, n+1]$, and that $P_{i}\left(x_{0}\right)$ holds if and only if $(\forall m<i) \neg f(m)=l_{1}$. Otherwise, let $p_{\langle k, c\rangle}=q_{k}$.

In the first case, $p_{\langle k, c\rangle}=q_{k}$ if and only if $l_{1}$ is not in the range of $f$. If $l_{1}$ is in the range of $f$, however, then the first case applies for only finitely many $c$, so if $c$ is sufficiently large then $p_{\langle k, c\rangle}=q_{k}$. Thus every type of $T$ appears in $X$. Furthermore, if we know whether $l_{1}$ is in the range of $f$, then we can determine which type $p_{\langle k, c\rangle}$ is, for any given $c \in \mathbb{N}$. By bounded $\Sigma_{1}^{0}$-comprehension, it follows that for any sequence $i_{0}, \ldots, i_{m}$, there is a sequence $j_{0}, \ldots, j_{m}$ such that $p_{i_{d}}=q_{j_{d}}$ for all $d \leqslant m$. It is now easy to see that $X$ satisfies the finite full amalgamation closure conditions.

We define an extension function approximation $g$ for $X$ as follows. Fix $p_{i}(\bar{x})$ and $\theta_{j}(\bar{x} \bar{y})$ consistent with $p_{i}(\bar{x})$. By effective quantifier elimination and the fact that a disjunction is implied by each of its disjuncts, we may assume that $\theta_{j}$ is a conjunction of literals. Let $k, c$ be such that $i=\langle k, c\rangle$. There are several cases.

(1) $p_{i}$ is the 0-type. Then we can find an $m$ such that $q_{m}$ contains $\theta_{j}$ and is of the form $s_{E,\left(l_{0}, \ldots, l_{n-1}\right)}$, where $n=|\bar{y}|$ and each $l_{j}$ is in $\mathbb{N}$, and let $g(i, j, s)=\langle m, 0\rangle$ for all $s \in \mathbb{N}$. 
(2) $p_{i}$ is an $m$-type for $m \geqslant 2$ that is defined to be $q_{k}$. Then we also have $p_{\langle h(k, j), c\rangle}=q_{h(k, j)}$, so let $g(i, j, s)=\langle h(k, j), c\rangle$ for all $s \in \mathbb{N}$.

(3) $p_{i}$ is an $m$-type for $m \geqslant 2$, but case 2 does not hold. Let $j^{\prime}$ be such that $\theta_{j^{\prime}}$ implies $\theta_{j}$ and the free variables of $\theta_{j^{\prime}}$ are $x_{0}, \ldots, x_{m}$ for some $m$ (for instance, $\theta_{j^{\prime}}$ can be obtained by adding conjuncts of the form $x_{l}=x_{l}$ to $\left.\theta_{j}\right)$. Then it follows from the definition of $X$ that $p_{\left\langle h\left(i, j^{\prime}\right), c\right\rangle}$ is obtained from $q_{h\left(i, j^{\prime}\right)}$ by the same process as $p_{i}$ is obtained from $q_{k}$, which implies that $p_{i} \cup\left\{\theta_{j}\right\} \subset p_{\left\langle h\left(k, j^{\prime}\right), c\right\rangle}$, so let $g(i, j, s)=\left\langle h\left(k, j^{\prime}\right), c\right\rangle$ for all $s \in \mathbb{N}$.

(4) $p_{i}$ is a 1-type and $p_{\langle h(k, j), c\rangle}$ is defined to be $q_{h(k, j)}$. Then $p_{i}=q_{k}$, so let $g(i, j, s)=\langle h(k, j), c\rangle$ for all $s \in \mathbb{N}$.

(5) None of the above cases hold. Then $p_{i}=q_{k}$ is a 1-type and $q_{h(k, j)}$ is of the form $s_{E,\left(\infty, l_{1}, \ldots, l_{n+1}\right)}\left(x_{0}, \ldots, x_{n+1}\right)$, where $\neg E(0,1)$. By our assumptions on $h$, it follows that $p_{i}$ is $r_{\infty}\left(x_{0}\right)$, and that $\theta_{j}\left(x_{0}, \ldots, x_{n+1}\right)$ does not include $x_{0}=$ $x_{1}$. Let $l$ be a number larger than all $d$ such that $\theta_{j}$ includes $P_{d}\left(x_{1}\right)$ and less than or equal to all $d$ such that $\theta_{j}$ includes $\neg P_{d}\left(x_{1}\right)$. Such an $l$ exists because $\theta_{j}$ is consistent with $T$. Let $j^{\prime}$ be such that $\theta_{j^{\prime}}$ is $\theta_{j} \wedge \neg P_{l}\left(x_{1}\right) \wedge P_{l-1}\left(x_{1}\right)$, where the last conjunct is omitted if $l=0$. If $(\forall m<s) \neg f(m)=l$, then let $g(i, j, s)=\left\langle h\left(k, j^{\prime}\right), 0\right\rangle$. Otherwise, let $g(i, j, s)=\left\langle h\left(k, j^{\prime}\right), s\right\rangle$.

In any case, it follows from the definition of $X$ that $\lim _{s} g(i, j, s)$ exists, that $p_{g(i, j, s)}\left\lceil s=p_{g(i, j, s+1)}\left\lceil s\right.\right.$ for all $s \in \mathbb{N}$, and that $p_{i} \cup\left\{\theta_{j}\right\} \subset p_{\lim _{s} g(i, j, s)}$.

Now suppose that $X$ has an extension function $G$. For each $n$, let $i, j$ be such that $p_{i}=r_{\infty}\left(x_{0}\right)$ and $\theta_{j}=P_{n-1}\left(x_{1}\right) \wedge \neg P_{n}\left(x_{1}\right)$ (or just $\neg P_{0}\left(x_{1}\right)$ if $n=0$ ). Then $G(i, j)$ must have the form $\langle k, c\rangle$ for some $c$ such that either $(\exists m<c) f(m)=n$, or $n$ is not in the range of $f$. Thus from $G$ we obtain the range of $f$ as a set.

\subsection{The reverse mathematics of extension functions}

From the reverse mathematical point of view, there are several versions of Theorem 6.4 that can be considered. We begin with the following simple facts.

Proposition 6.6. The following are provable in $\mathrm{RCA}_{0}$.

(1) For every model $\mathcal{A}$, there is an enumeration of $\mathbb{T}(\mathcal{A})$ with an extension function and the same properties as in Proposition 5.13.

(2) If two lists of types are equal as sets of types and one has an extension function approximation, then so does the other.

(3) If two lists of types are equal as sets of types and one has an extension function, then the other has an extension function approximation.

(4) For every model $\mathcal{A}$, every enumeration of $\mathbb{T}(\mathcal{A})$ has an extension function approximation.

Proof. (1) The enumeration described in the proof of Proposition 5.13 clearly has an extension function.

(2) Suppose that $Y=\left\{q_{i}\right\}_{i \in \mathbb{N}}$ has an extension function approximation $g$ and that $X=\left\{p_{i}\right\}_{i \in \mathbb{N}}$ is equal to $Y$ as a set of types. Given $i$ and an index $j$ for an $(n+c)$ ary formula $\theta$ consistent with the $n$-type $p_{i}$, proceed as follows. Let $f(i, j, 0)=0$, let $k_{0}=0$, and let $\varphi_{0}=\theta$. Given $f(i, j, s)$, let $k_{s+1}$ be the least $k$ such that $q_{k}$ is an $n$-type with $q_{k} \uparrow s=p_{i} \uparrow s$. If $k_{s+1}=k_{s}$ then let $\varphi_{s+1}=\varphi_{s}$. Otherwise, let $\varphi_{s+1}$ be the conjunction of the formulas in $p_{f(i, j, s)} \uparrow s$ and $\theta$. Let $m$ be the index of $\varphi_{s+1}$, and let $f(i, j, s+1)$ be the least $l$ such that $p_{l}\left\lceil s+1=q_{g(k, m, s+1)}\lceil s+1\right.$. It is straightforward to check that $f$ is an extension function approximation for $X$, 
TABLE 6.1. Versions of Theorem 6.4

\begin{tabular}{|c|c|c|c|c|c|}
\hline & $\begin{array}{c}\text { p'wise } \\
\text { free } \\
\text { amalg. }\end{array}$ & $\begin{array}{c}\text { finite } \\
\text { free } \\
\text { amalg. }\end{array}$ & $\begin{array}{c}1 \text {-pt. } \\
\text { full } \\
\text { amalg. }\end{array}$ & $\begin{array}{c}\text { p'wise } \\
\text { full } \\
\text { amalg. }\end{array}$ & $\begin{array}{c}\text { finite } \\
\text { full } \\
\text { amalg. }\end{array}$ \\
\hline$X$ has pairwise amalg. & $\sqrt{ }$ & $\sqrt{ }$ & 2 & $\sqrt{ }$ & $\sqrt{ }$ \\
\hline$X$ has finite amalg. & 2 & $\sqrt{ }$ & 2 & 2 & $\sqrt{ }$ \\
\hline$\exists$ listing w/ finite amalg. & 3 & $\sqrt{ }$ & 2 & 2 & $\sqrt{ }$ \\
\hline$\exists$ model & 3 & 1 & 2 & 3 & 1 \\
\hline$\exists$ 1-pt. homogeneous model & $\mathrm{X}$ & $\mathrm{X}$ & 2 & 3 & 1 \\
\hline$\exists$ 1-homogeneous model & $\mathrm{X}$ & $\mathrm{X}$ & 2 & 3 & 1 \\
\hline$\exists$ homogeneous model & $\mathrm{X}$ & $\mathrm{X}$ & 2 & 2 & 1 \\
\hline$\exists$ listing w/ EF & 4 & 1 & 5 & 4 & 1 \\
\hline$\exists$ listing w/ EF + p'wise am. & 4 & 1 & 2 & 4 & 1 \\
\hline$\exists$ listing w/ EF + fin. am. & 3 & 1 & 2 & 2 & 1 \\
\hline
\end{tabular}

using the fact that for each $k$ there is a least $l$ such that $p_{k}=q_{l}$ (and vice-versa). This fact follows from $\mathrm{I \Pi}_{1}^{0}$, which is provable in $\mathrm{RCA}_{0}$.

(3) follows from (2), while (4) follows from (1) and (3).

We summarize what we know about other versions of Theorem 6.4 in Table 6.1. The column heading describes which closure conditions we assume the list of types $X=\left\{p_{i}\right\}_{i \in \mathbb{N}}$ satisfies. When a row heading asserts the existence of a model $\mathcal{A}$, we mean that $X$ is an enumeration of $\mathbb{T}(\mathcal{A})$. When a row heading asserts the existence of a listing, we mean that there is a list of types $Y=\left\{q_{i}\right\}_{i \in \mathbb{N}}$ with the stated property such that $X$ and $Y$ are equal as sets of types. For a given column $C$ and row $R$, let $C \Rightarrow R$ be the principle stating that if $X$ has the closure conditions given by the heading of $C$ and has an extension function approximation, then the statement in the heading of $R$ holds. (When the heading of $R$ mentions amalgamation, we take it in the same sense (free or full) as in the heading of $C$. Note that, by Proposition 5.10, we do not need a separate "there exists a listing with pairwise amalgamation" row, as such a row would be the same as the first row in our chart.)

The entry in row $R$ and column $C$ in Table 6.1 describes the strength of the principle $C \Rightarrow R$. A $\sqrt{ }$ means that the principle is true by definition, and an $\mathrm{X}$ means that it is false. The meanings of the number codes are as follows.

- A 1 means that the principle is true in $\mathrm{RCA}_{0}$.

- A 2 means that the principle is equivalent to $\mathrm{I} \Sigma_{2}^{0}$ over $\mathrm{RCA}_{0}$.

- A 3 means that the principle is provable from $\Pi_{1}^{0} \mathrm{GA}$ over $\mathrm{RCA}_{0}$ and is equivalent to $\mathrm{I} \Sigma_{2}^{0}$ over $\mathrm{RCA}_{0}+\mathrm{B} \Sigma_{2}^{0}$ (and hence not provable in $\mathrm{RCA}_{0}$, or even $\left.\mathrm{RCA}_{0}+\mathrm{B} \Sigma_{2}^{0}\right)$.

- A 4 means that the principle is provable from $\Pi_{1}^{0} \mathrm{GA}$ over $\mathrm{RCA}_{0}$, but its strength is otherwise unknown.

- A 5 means that the principle is provable from $\mathrm{I}_{2}^{0}$ over $\mathrm{RCA}_{0}$, but its strength is otherwise unknown.

The proof that entries marked 1 are true in $\mathrm{RCA}_{0}$ is done simultaneously with the proof that entries marked 3 or 4 are provable from $\Pi_{1}^{0} \mathrm{GA}$, and employs Shore blocking. We use versions of a single example to obtain $\mathrm{I} \Sigma_{2}^{0}$ from the entries marked 
TABle 6.2. Changes to Table 6.1 in the extension function case

\begin{tabular}{|c|c|c|c|c|c|}
\hline & $\begin{array}{c}\text { p'wise } \\
\text { free } \\
\text { amalg. }\end{array}$ & $\begin{array}{c}\text { finite } \\
\text { free } \\
\text { amalg. }\end{array}$ & $\begin{array}{c}1 \text {-pt. } \\
\text { full } \\
\text { amalg. }\end{array}$ & $\begin{array}{c}\text { p'wise } \\
\text { full } \\
\text { amalg. }\end{array}$ & $\begin{array}{c}\text { finite } \\
\text { full } \\
\text { amalg. }\end{array}$ \\
\hline$\exists$ listing w/ EF & $\sqrt{ }$ & $\sqrt{ }$ & $\sqrt{ }$ & $\sqrt{ }$ & $\sqrt{ }$ \\
\hline$\exists$ listing w/ EF + p'wise am. & $\sqrt{ }$ & $\sqrt{ }$ & 2 & $\sqrt{ }$ & $\sqrt{ }$ \\
\hline$\exists$ listing w/ EF + fin. am. & 3 & $\sqrt{ }$ & 2 & 2 & $\sqrt{ }$ \\
\hline
\end{tabular}

2 and from the entries marked 3 together with $\mathrm{B} \Sigma_{2}^{0}$. We give two charts (Tables 6.4 and 6.5) summarizing the justifications of the correctness of Table 6.1 in the proof of Theorem 6.13 below.

We can also think of this chart with $C \Rightarrow R$ reinterpreted to mean that if $X$ has the closure conditions given by the heading of $C$ and has an extension function (rather than an extension function approximation), then the statement in the heading of $R$ holds. In this case, the chart remains exactly the same, except for the third to last row, which is now all $\sqrt{ }$ 's; the next to last row, where the 1's and 4 's are now $\sqrt{ }$ 's; and the last row, where the 1 's are now $\sqrt{ }$ 's. We show these changed lines in Table 6.2. The justifications of correctness in this case are also given by the charts in the proof of Theorem 6.13.

As noted in Chapter 3, the definition of $\Pi_{1}^{0} \mathrm{GA}$ is intended to capture the reverse mathematical behavior of the principles marked with a 3 in a more combinatorial way. However, we do not know whether these principles are in fact equivalent to $\Pi_{1}^{0} \mathrm{GA}$.

In the remainder of this chapter, we prove theorems that, together with results in preceding chapters, are used in Theorem 6.13 below to verify the correctness of both versions of our chart. We begin by defining an auxiliary notion of type amalgamation that is implied by full amalgamation and, in the presence of $\Pi_{1}^{0} \mathrm{GA}$, also by pairwise amalgamation. We later give a proof establishing the provability in $\mathrm{RCA}_{0}$ of the entries labeled 1 in our chart, the provability from $\mathrm{I} \Sigma_{2}^{0}$ of the entries labeled 2 or 5 , and the provability from $\Pi_{1}^{0} \mathrm{GA}$ of the entries labeled 3 or 4. In this proof, instead of working with finite or pairwise amalgamation directly, we work with this auxiliary notion, which is designed to fit a finite injury priority construction (with Shore blocking). Here and below, we use the bracket notation introduced in Remark 5.9.

Definition 6.7. Let $X=\left\{p_{i}\left(\bar{z}_{i}\right)\right\}_{i \in \mathbb{N}}$ be a list of types of a theory $T$. Let $a_{i}=\left|\bar{z}_{i}\right|$. Let $R$ be the set of all $(i, n)$ with $n \leqslant a_{i}$. Let $x_{i}$ and $y_{i, n, j}$ for $i, n, j \in \mathbb{N}$ be pairwise distinct variables. For $(i, n) \in R$, let

$$
q_{i, n}=p_{i}\left[x_{0}, \ldots, x_{n-1}, y_{i, n, 0}, \ldots, y_{i, n, a_{i}-n-1}\right] .
$$

(So if $(i, n)$ and $\left(i^{\prime}, n^{\prime}\right)$ are distinct elements of $R$, then the first $\min \left(n, n^{\prime}\right)$ many variables in $q_{i, n}$ and $q_{i^{\prime}, n^{\prime}}$ are the same, but the other variables in $q_{i, n}$ are pairwise distinct from the other variables in $q_{i^{\prime}, n^{\prime}}$.) For $F \subseteq R$, let $q_{F}=\left\{q_{i, n}:(i, n) \in F\right\}$. Recall that our definition of consistency of a finite set of types requires that there be a single type in the set containing all the variables shared by any two types in the set. If $F \subset R$ is finite, then this is the case for $q_{F}$, so it makes sense to speak of the consistency of $q_{F}$. 
(1) $X$ satisfies the eventual full type amalgamation condition if there are finite $F_{0}, F_{1}, \ldots \subset R$ such that the following hold.

(a) For each $(i, n) \in R$, we have $(i, n) \in F_{s}$ for all sufficiently large $s$.

(b) There are infinitely many $s$ such that, for each $S \subseteq F_{s}$ for which $q_{S}$ is consistent, there is a $p_{j}$ amalgamating all the types in $q_{S}$.

(2) $X$ satisfies the eventual free type amalgamation condition if the above holds for $\{(i, 0): i \in \mathbb{N}\}$ in place of $R$.

(3) X satisfies the eventual full amalgamation closure conditions (resp. eventual free amalgamation closure conditions) if it satisfies the conditions in Theorem 5.1 with pairwise full type amalgamation replaced by eventual full type amalgamation (resp. eventual free type amalgamation).

Clearly, if a list of types of a theory satisfies the finite full (resp. free) amalgamation closure conditions then it satisfies the eventual full (resp. free) amalgamation closure conditions. For pairwise amalgamation, we have the following fact.

LEMMA 6.8. The following is provable in $\mathrm{RCA}_{0}+\Pi_{1}^{0} \mathrm{GA}$. If a list of types of a theory satisfies the pairwise full (resp. free) amalgamation closure conditions then it satisfies the eventual full (resp. free) amalgamation closure conditions.

PROOF. We prove the lemma for full amalgamation, as the free case is similar but simpler. We argue in $\mathrm{RCA}_{0}+\Pi_{1}^{0} \mathrm{GA}$. We adopt the notation of Definition 6.7. Let $X=\left\{p_{i}\left(\bar{z}_{i}\right)\right\}_{i \in \mathbb{N}}$ be a list of types of a theory $T$ satisfying the pairwise full amalgamation closure conditions. Let $w_{i, j}$ for $i, j \in \mathbb{N}$ be pairwise distinct variables that are also distinct from each $x_{i}$ and each $y_{i, n, j}$. For $(i, n) \in R$, let $r_{i, n}=q_{i, n}\left\lceil x_{0}, \ldots, x_{n-1}\right.$, and let $l_{s}(i, n)$ be the least $l$ such that $r_{l, n}\left\lceil s=r_{i, n}\lceil s\right.$ Let $E \subset R$ be finite. We say that a pair $(d, s)$ provides amalgamators for $E$ if for each $(i, n) \in E$, the type $p_{d}$ contains

$$
p_{i}\left[w_{l_{s}(i, 1), 0}, \ldots, w_{l_{s}(i, n), n-1}, y_{i, n, 0}, \ldots, y_{i, n, a_{i}-n-1}\right] .
$$

This property is $\Pi_{1}^{0}$, so there is a $\Delta_{0}^{0}$ predicate $P$ such that $(d, s)$ provides amalgamators for $E$ if and only if $(\forall x) P(d, s, E, x)$.

Suppose $(d, s)$ provides amalgamators for $E$, and let $S \subseteq E$ be such that $q_{S}$ is consistent. If $(i, n),\left(i^{\prime}, n^{\prime}\right) \in S$, then for all $k \leqslant \min \left(n, n^{\prime}\right)$, we have $r_{i, k}=r_{i^{\prime}, k}$, and hence $l_{s}(i, k)=l_{s}\left(i^{\prime}, k\right)$. So if we choose $(i, n) \in S$ with the largest $n$ and let $p_{j}$ be the type obtained from $p_{d}$ by first passing to a subtype that does not include the variables $x_{0}, \ldots, x_{n-1}$ and then replacing the variables $w_{l_{s}(i, 1), 0}, \ldots, w_{l_{s}(i, n), n-1}$ by $x_{0}, \ldots, x_{n-1}$, then $p_{j}$ amalgamates the types in $q_{S}$. So the idea is to use $\Pi_{1}^{0} \mathrm{GA}$ to find such $(d, s)$ for larger and larger sets $E$.

Let $\left(E_{0}, d_{0}\right),\left(E_{1}, d_{1}\right), \ldots$ list all pairs consisting of a finite subset of $R$ and a natural number, such that each such pair is listed infinitely often. For $\sigma \in 2^{<\mathbb{N}}$, proceed as follows to define a tuple $\left(E_{\sigma}, d_{\sigma}, s_{\sigma}, t_{\sigma}\right)$. Let $s_{\sigma}$ be the largest $s$ such that $\sigma(3 s)=1$, or 0 if there is no such $s$. Let $t_{\sigma}$ be the largest $t$ such that $\sigma(3 t+1)=1$, or 0 if there is no such $t$. Let $k_{0}<\cdots<k_{m-1}$ be the numbers such that $\sigma\left(3 k_{i}+2\right)=1$. Let $\left(E^{0}, d^{0}\right)=(\emptyset, 0)$. Given $\left(E^{i}, d^{i}\right)$, proceed as follows. If $E_{k_{i}} \supseteq E^{i}$ and $\left(\forall x<t_{\sigma}\right) P\left(d_{k_{i}}, s_{\sigma}, E_{k_{i}}, x\right)$, then let $\left(E^{i+1}, d^{i+1}\right)=\left(E_{k_{i}}, d_{k_{i}}\right)$, and otherwise let $\left(E^{i+1}, d^{i+1}\right)=\left(E^{i}, d^{i}\right)$. Finally, let $\left(E_{\sigma}, d_{\sigma}\right)=\left(E^{m}, d^{m}\right)$. Note that $\left(\forall x<t_{\sigma}\right) P\left(d_{\sigma}, s_{\sigma}, E_{\sigma}, x\right)$.

We now define some uniformly $\Pi_{1}^{0}$ dense sets. (As in the definition of $\Pi_{1}^{0} \mathrm{G}$ and $\Pi_{1}^{0} \mathrm{GA}$ in Chapter 3, we refer to these $\Pi_{1}^{0}$ properties as sets and use set notation for 
convenience, but we are not asserting their existence as sets in $\mathrm{RCA}_{0}$.) We begin with the sets $D_{c}$ of all $\tau$ such that $|\tau|>c$ and $\left(d_{\tau}, s_{\tau}\right)$ provides amalgamators for $E_{\tau}$. These sets are uniformly $\Pi_{1}^{0}$; we claim each $D_{c}$ is also dense. To see that this is the case, fix $c$ and $\sigma$, and let $k_{0}<\cdots<k_{m-1}$ be as above. It is provable in $\mathrm{RCA}_{0}$ that there is a $t \geqslant|\sigma|$ such that, for each $i<m$, if $(\exists x) \neg P\left(d_{k_{i}}, s_{\sigma}, E_{k_{i}}, x\right)$ then $(\exists x<t) \neg P\left(d_{k_{i}}, s_{\sigma}, E_{k_{i}}, x\right)$. Let $\tau \succ \sigma$ be such that $|\tau|>c$ and $\tau(j)=1$ if and only if $\sigma(j)=1$ or $j=3 t+1$. Then $t_{\tau}=t$ and $s_{\tau}=s_{\sigma}$, while $\left(E_{\tau}, d_{\tau}\right)=\left(E_{k_{i}}, d_{k_{i}}\right)$ for some $i<m$ (or $\left.\left(E_{\tau}, d_{\tau}\right)=(\emptyset, 0)\right)$. Thus the fact that $\left(\forall x<t_{\tau}\right) P\left(d_{\tau}, s_{\tau}, E_{\tau}, x\right)$ implies that $(\forall x) P\left(d_{\tau}, s_{\tau}, E_{\tau}, x\right)$, and hence $\left(d_{\tau}, s_{\tau}\right)$ provides amalgamators for $E_{\tau}$.

Now let $C_{(i, n)}$ for $(i, n) \in R$ consist of all $\tau$ such that $(i, n) \in E_{\rho}$ for all $\rho \succcurlyeq \tau$. The $C_{(i, n)}$ are uniformly $\Pi_{1}^{0}$; we claim that each $C_{(i, n)}$ is also dense. To see that this is the case, fix $(i, n) \in R$ and $\sigma$, and let $k_{0}<\cdots<k_{m-1}$ be as above. Let $E=\bigcup_{j<m} E_{k_{j}} \cup\{(i, n)\}$. For each $(j, k) \in E$, the value of $l_{s}(j, k)$ changes at most $j$ many times, so it is provable in $\mathrm{RCA}_{0}$ that there is an $s \geqslant|\sigma|$ such that $l_{u}(j, k)=l_{s}(j, k)$ for all $u \geqslant s$ and all $(j, k) \in E$. Write $l(j, k)$ for $l_{s}(j, k)$. As above, there is a $t \geqslant|\sigma|$ such that, for each $i<m$, if $(\exists x) \neg P\left(d_{k_{i}}, s, E_{k_{i}}, x\right)$ then $(\exists x<t) \neg P\left(d_{k_{i}}, s, E_{k_{i}}, x\right)$. Let $\nu \succ \sigma$ be such that $\nu(j)=1$ if and only if $\sigma(j)=1$ or $j=3 s$ or $j=3 t+1$. Then for each $(j, k) \in E_{\nu}$, the type $p_{d_{\nu}}$ contains

$$
p_{j}\left[w_{l(j, 1), 0}, \ldots, w_{l(j, k), k-1}, y_{j, k, 0}, \ldots, y_{j, k, a_{j}-k-1}\right] .
$$

By passing to a subtype if necessary, we may assume that $p_{d_{\nu}}$ does not contain the variable $w_{l(i, c+1), c}$ for $c<n$ unless there is a $(j, k) \in E_{\nu}$ such that $c<k$ and $l(j, c+1)=l(i, c+1)$, in which case $r_{j, c+1}=r_{i, c+1}$. Thus $p_{d_{\nu}}$ is consistent with

$$
p_{i}\left[w_{l(i, 1), 0}, \ldots, w_{l(i, n), n-1}, y_{i, n, 0}, \ldots, y_{i, n, a_{i}-n-1}\right],
$$

and hence there is a type $p_{d}$ amalgamating these two types. Let $k>|\nu|$ be such that $\left(E_{k}, d_{k}\right)=\left(E_{\nu} \cup\{(i, n)\}, d\right)$, and let $\tau \succ \nu$ be such that $\tau(j)=1$ if and only if $\nu(j)=1$ or $j=3 k+2$. Then it is easy to check that $E_{\tau}=E_{\nu} \cup\{(i, n)\}$, and that if $\rho \succcurlyeq \tau$, then the definition of $E_{\rho}$ proceeds in the same way as the definition of $E_{\tau}$ for its first $m+1$ many steps, whence $(i, n) \in E_{\tau} \subseteq E_{\rho}$.

Now let $g_{0}, g_{1}, \ldots$ be as in $\Pi_{1}^{0} \mathrm{GA}$ for the uniformly $\Pi_{1}^{0}$ dense sets $D_{m}$ for $m \in \mathbb{N}$ and $C_{(i, n)}$ for $(i, n) \in R$. Let $\sigma_{0}, \sigma_{1}, \ldots$ be a listing without repetitions of all $\sigma$ such that there is a $t$ with $\sigma \prec g_{t}$. Let $F_{u}=E_{\sigma_{u}}$. Let $(i, n) \in R$. Then there are $k$ and $s$ such that for all $t \geqslant s$, we have $g_{t} \uparrow k=g_{s} \uparrow k \in C_{(i, n)}$, and hence $(i, n) \in F_{u}$ for almost all $u$. For each $m$, there are $k$ and $s$ such that $g_{s} \uparrow k \in D_{m}$, so there are infinitely many $F_{u}$ such that some $(d, v)$ provides amalgamators for $F_{u}$. As discussed above, for any such $u$ and any $S \subseteq F_{u}$ such that $q_{S}$ is consistent, there is a $p_{j}$ amalgamating all the types in $q_{S}$. Thus $X$ satisfies the eventual full type amalgamation condition.

The next lemma gives us a convenient way to build a model with a specified type spectrum by computably approximating the types of initial segments of the domain of the model.

LEMma 6.9. The following is provable in $\mathrm{RCA}_{0}$. Let $X=\left\{p_{i}\right\}_{i \in \mathbb{N}}$ be a list of types of a theory T. Let $q_{i, n}$ be as in Definition 6.7. Suppose that $X$ satisfies the first four conditions in Theorem 5.1, and there is a binary partial function $f$ with the following properties for all $n, s$, and $k$.

(i) $p_{f(n, s)}$ is an $n$-type in the variables $x_{0}, \ldots, x_{n-1}$ whenever defined. 
(ii) $p_{f(n, s)} \uparrow s=p_{f(n, s+k)} \uparrow s$ whenever these are defined.

(iii) $p_{f(n, s)} \uparrow s \subseteq p_{f(n+1, s)} \uparrow s$ whenever these are defined.

(iv) $f(n, s)$ is defined for all sufficiently large $s \in \mathbb{N}$ and $\lim _{s} f(n, s)$ exists. We denote this limit by $f(n)$. (Note that this notation is purely an abbreviation for the limit notation; we cannot in general prove that the function taking $n$ to $f(n)$ exists.)

(v) For each $(i, n) \in R$, if $p_{f(n)} \subseteq q_{i, n}$ then there exist $m \in \mathbb{N}$ and distinct $j_{0}, \ldots, j_{a_{i}-n-1} \in[n, m-1]$ such that

$$
p_{i}\left[x_{0}, \ldots, x_{n-1}, x_{j_{0}}, \ldots, x_{j_{a_{i}-n-1}}\right] \subseteq p_{f(m)} .
$$

Then the following hold.

(1) If $X$ satisfies the finite full type amalgamation condition, then there is a homogeneous model $\mathcal{M}$ such that $X$ is an enumeration of the type spectrum of $\mathcal{M}$.

(2) If $X$ satisfies the pairwise full type amalgamation condition, then there is a 1 -homogeneous model $\mathcal{M}$ such that $X$ is an enumeration of the type spectrum of $\mathcal{M}$.

(3) Suppose that we weaken condition (v) by requiring it to hold only for $n=0$. Then there is a model $\mathcal{M}$ such that $X$ is an enumeration of the type spectrum of $\mathcal{M}$.

Proof. (1) We obtain a homogeneous model $\mathcal{M}$ such that $X$ is an enumeration of $\mathbb{T}(\mathcal{M})$ as follows. First, define an equivalence relation $E$ on $\mathbb{N}$. For each $i, j \in \mathbb{N}$, let $n=\max (i, j)+1$. Then there is an $s \in \mathbb{N}$ such that either $x_{i}=x_{j} \in p_{f(n, s)}$ or $x_{i} \neq x_{j} \in p_{f(n, s)}$. Let $E(i, j)$ hold if and only if the first case obtains. Let the domain $M$ of $\mathcal{M}$ consist of all $i \in \mathbb{N}$ such that $(\forall j<i) \neg E(i, j)$. To specify the satisfaction predicate of $\mathcal{M}$, it is enough to say whether or not $\varphi^{\mathcal{M}}\left(m_{0}, \ldots, m_{k-1}\right)$ holds for each formula $\varphi\left(y_{0}, \ldots, y_{k-1}\right)$ in the language of $T$ and each tuple $\left(m_{0}, \ldots, m_{k-1}\right)$ of distinct elements of $M$, and then verify that our definition has the properties of a satisfaction predicate. Given such a $\varphi$ and $m_{0}, \ldots, m_{k-1}$, let $n=\max _{i<k} m_{i}+1$. Then there is an $s \in \mathbb{N}$ such that either $\varphi\left(x_{m_{0}}, \ldots, x_{m_{k-1}}\right) \in p_{f(n, s)}$ or $\neg \varphi\left(x_{m_{0}}, \ldots, x_{m_{k-1}}\right) \in p_{f(n, s)}$. Let $\varphi^{\mathcal{M}}\left(m_{0}, \ldots, m_{k-1}\right)$ hold if and only if the first case obtains.

Then $\varphi^{\mathcal{M}}$ holds for all $\varphi \in T$, since every type in $X$ contains $T$. It is also easy to check that the predicate defined in this way is deductively closed and consistent, and hence is a satisfaction predicate, so $\mathcal{M}$ is a model of $T$. By the properties of $f$, the fact that $X$ is closed under subtypes, and Remark 5.2, every $M$-tuple realizes a type in $X$. For each $p_{i} \in X$, we have $p_{f(0)} \subseteq p_{i}$, so by the fifth property of $f$, the type $p_{i}$ is realized in $\mathcal{M}$.

It is easy to see that in the definition of homogeneity of a model (Definition 4.1(iii)), restricting attention to $M$-tuples of distinct elements yields an equivalent definition of homogeneity. Thus, to show that $\mathcal{M}$ is homogeneous, let $\bar{a}_{0} \equiv$ $\bar{b}_{0}, \ldots, \bar{a}_{n} \equiv \bar{b}_{n}$ and $\bar{c}_{0}, \ldots, \bar{c}_{n}$ be $M$-tuples of distinct elements (by which we mean that each $\bar{a}_{i}, \bar{b}_{i}$, and $\bar{c}_{i}$ is a tuple of distinct elements, though some of these tuples could have elements in common). Let $m$ be larger than all of the elements of these tuples. Let $k_{i}=\left|\bar{b}_{i}\right|$ and $l_{i}=\left|\bar{c}_{i}\right|$. Write $\bar{b}_{i}=\left(b_{i, 0} \ldots, b_{i, k_{i}-1}\right)$. Let $z_{i, j}$ for $i \leqslant n$ 
and $j<l_{i}$ be pairwise distinct variables that are also pairwise distinct from the $x_{i}$. Let $r_{i}\left(x_{b_{i, 0}}, \ldots, x_{b_{i, k_{i}-1}}, z_{i, 0}, \ldots, z_{i, l_{i}-1}\right)$ be the type of $\bar{a}_{i} \bar{c}_{i}$. The sequence of types consisting of $p_{f(m)}$ and $r_{i}$ for $i \leqslant n$ is basically consistent, so there is a type

$$
p_{j}\left(x_{0}, \ldots, x_{m-1}, z_{0,0}, \ldots, z_{0, l_{0}-1}, \ldots, z_{n, 0}, \ldots, z_{n, l_{n}-1}\right)
$$

amalgamating these types.

Let $\bar{b}$ be the result of removing from $\bar{b}_{0} \ldots \bar{b}_{n}$ any $b_{i, j}$ for which there are an $i^{\prime}<i$ and a $j^{\prime}$ such that $b_{i^{\prime}, j^{\prime}}=b_{i, j}$. Let $\bar{x}$ be the result of removing from the list $x_{b_{0,0}} \ldots x_{b_{0, k_{0}-1}} \ldots x_{b_{n, 0}} \ldots x_{b_{n, k_{n}-1}}$ any $x_{i, j}$ for which there are an $i^{\prime}<i$ and a $j^{\prime}$ such that $x_{i^{\prime}, j^{\prime}}$ is the same variable as $x_{i, j}$. It now follows from the fifth property of $f$ that there are $\bar{d}_{0}, \ldots, \bar{d}_{n} \in M$ such that $\bar{b} \bar{d}_{0} \ldots \bar{d}_{n}$ realizes

$$
p_{j} \uparrow \bar{x} z_{0,0} \ldots z_{0, l_{0}-1} \ldots z_{n, 0} \ldots z_{0, l_{n}-1} .
$$

Then $\bar{a}_{i} \bar{c}_{i} \equiv \bar{b}_{i} \bar{d}_{i}$ for all $i \leqslant n$.

(2) The above argument works in this case as well, except that in the last two paragraphs, we take $n=0$, which makes the amalgamation performed in that paragraph an instance of pairwise full type amalgamation.

(3) The same argument again works in this case, ignoring the last two paragraphs.

We are now ready to establish a theorem that gives us the provability in $\mathrm{RCA}_{0}$ of the entries labeled 1 in our chart, the provability from $\mathrm{I} \Sigma_{2}^{0}$ of the entries labeled 2 or 5 , and the provability from $\Pi_{1}^{0} \mathrm{GA}$ of the entries labeled 3 or 4 .

THEOREM 6.10. The following are provable in $\mathrm{RCA}_{0}$. Let $X=\left\{p_{i}\right\}_{i \in \mathbb{N}}$ be a list of types of a theory $T$, such that $X$ has an extension function approximation.

(1) If $X$ satisfies the finite full amalgamation closure conditions then there is a homogeneous model $\mathcal{M}$ such that $X$ is an enumeration of the type spectrum of $\mathcal{M}$.

(2) If $X$ satisfies the finite free amalgamation closure conditions then there is a model $\mathcal{M}$ such that $X$ is an enumeration of the type spectrum of $\mathcal{M}$.

(3) If $\Pi_{1}^{0} \mathrm{GA}$ holds and $X$ satisfies the pairwise full amalgamation closure conditions then there is a 1-homogeneous model $\mathcal{M}$ such that $X$ is an enumeration of the type spectrum of $\mathcal{M}$.

(4) If $\Pi_{1}^{0} \mathrm{GA}$ holds and $X$ satisfies the pairwise free amalgamation closure conditions then there is a model $\mathcal{M}$ such that $X$ is an enumeration of the type spectrum of $\mathcal{M}$.

ProOF. We do the full amalgamation case, as the free amalgamation case is similar. By Lemma 6.8 and the comment preceding that lemma, we may assume that $X$ satisfies the eventual full amalgamation closure conditions. From now on, this is the assumption we use, so we prove (1) and (3) simultaneously. It is enough to work in $\mathrm{RCA}_{0}$ and build a function $f$ as in Lemma 6.9 . Let $R$ and $F_{0}, F_{1}, \ldots$ be as in Definition 6.7.

Suppose that we build a list of types $Y$ as follows. For each $p_{i}$ in turn, add all of its subtypes (including $p_{i}$ itself), and all types obtained from these subtypes by changes of variables, to $Y$, in an effective way. Let $I$ be the set of all $j$ such that the $j$ th type added to $Y$ is one of the original $p_{i}$ 's. Then $X$ and $Y$ contain the same types, and, by Proposition 6.6, $Y$ also has an extension function approximation. Thus, by replacing $X$ with $Y$, we may assume that $X$ is closed under sequences of 
variable substitutions, and indeed that there is an effective procedure that, for each $p_{i}$ and each sequence $s$ of types obtained from subtypes of $p_{i}$ by a given change of variables, determines a sequence of elements of $X$ equal to $s$. We may also redefine $R$ to contain only those $(i, n)$ such that $i \in I$. Having property (v) of $f$ hold for such $(i, n)$ suffices for the proof of Lemma 6.9 to go through, and we still have sets $F_{0}, F_{1}, \ldots$ as in Definition 6.7 .

Let $g$ be an extension function approximation for $X$, relative to a listing $\varphi_{0}, \varphi_{1}, \ldots$ of the formulas in the language of $T$. We may assume that $g$ is defined so that $p_{g(i, j, s)}$ always contains $\varphi_{j}$. Furthermore, if for each $i$ and $j$ such that $\varphi_{j}(\bar{w} \bar{z})$ is consistent with $p_{i}(\bar{w})$, we replace each $g(i, j, s)$ by the least $k$ such that $p_{k}\left\lceil s=\left(p_{g(i, j, s)}\lceil\bar{w} \bar{z})\lceil s\right.\right.$, then it is easy to check that we still have an extension function approximation. Thus we may assume that for such $j$ and $i$, each $p_{g(i, j, s)}$ is a $|\bar{w} \bar{z}|$-type in the variables $\bar{w} \bar{z}$.

We ensure properties (i)-(iii) of $f$ as we go along the stage-by-stage construction. We think of the remaining two properties as split into requirements. For (iv), there is a requirement $Q_{n}$ for each $n \in \mathbb{N}$, and for (v) a requirement $R_{i, n}$ for each $i, n \in R$. We satisfy these requirements by a finite injury priority construction. Not all finite injury constructions carry through in $\mathrm{RCA}_{0}$, however. The typical issue is the following. There is no problem in verifying that a given requirement eventually stops acting, given that there is a stage $s$ at which all stronger priority requirements have stopped acting. However, concluding that this fact implies that, for each requirement, such a stage $s$ exists might require more induction than is available in $\mathrm{RCA}_{0}$. (The issue of how much induction is needed for various computability theoretic constructions has been extensively analyzed by the research program known as reverse recursion theory; see e.g. Mytilinaios [37].) To avoid this issue, we use Shore blocking. The use of blocking in the reverse mathematics of model theory is quite natural, since a finite list of requirements, each involving a particular type, can often be combined into a single requirement involving an amalgamation of the original types. For other examples of this technique, see [23].

In our case, the $Q$-requirements block naturally, but the $R$-requirements need some care. Satisfying a single $R$-requirement in the absence of injuries from stronger priority requirements is no problem, if we have at least pairwise full type amalgamation. However, satisfying an arbitrary block of finitely many $R$-requirements at once requires finite full type amalgamation. So instead of satisfying our $R$ requirements directly, we make use of the sets $F_{0}, F_{1}, \ldots$ from Definition 6.7 and satisfy requirements $S_{s}$ saying that there is a $t \geqslant s$ such that $R_{i, n}$ is satisfied for every $(i, n) \in F_{t}$ with $n \leqslant s$. Satisfying the $S_{s}$ is clearly enough to satisfy all $R_{i, n}$, but the $S$-requirements have the advantage that satisfying $S_{s}$ is enough to satisfy $S_{u}$ for all $u \leqslant s$.

For each $c, s \in \mathbb{N}$, we define a number $b_{c, s}$. At stage $s$, the $S$-requirements we attempt to satisfy are $S_{b_{0, s}}, S_{b_{1, s}}, \ldots$ For each $S_{b_{c, s}}$, we need numbers from which to draw the witnesses required to satisfy the appropriate $R$-requirements (i.e., the numbers $j_{i}$ mentioned in property (v) of $f$ ). So we define $b_{c+1, s}$ so that $b_{c+1, s}-b_{c, s}$ is at least as large as the total number of witnesses we might need, and draw our witnesses for $S_{b_{c, s}}$ from $\left[b_{c, s}, b_{c+1, s}\right)$. (We define $b_{0, s}=0$ for all $s$.)

At stage $s$ of our construction, the cth block of requirements consists of $S_{b_{c, s}}$ and the $Q_{n}$ such that $n \in\left(b_{c, s}, b_{c+1, s}\right]$. We try to satisfy all requirements in this block simultaneously, by defining $f\left(b_{c+1, s}, s\right)$ to attempt to satisfy $S_{b_{c, s}}$, defining $f(n, s)$ 
for $n \in\left(b_{c, s}, b_{c+1, s}\right)$ so that $p_{f(n, s)}$ is the appropriate subtype of $p_{f\left(b_{c+1, s}, s\right)}$ (which we can do because of the effective closure under sequences of variable substitutions discussed in the second paragraph of this proof), and then attempting to preserve these definitions. Of course, we also attempt to ensure that $p_{f\left(b_{c, s}, s\right)}$ is a subtype of $p_{f\left(b_{c+1, s}, s\right)}$.

When we say that we define $f\left(b_{c+1, s}, s\right)$ to attempt to satisfy $S_{b_{c, s}}$, we mean the following. Say that $R_{i, n}$ is relevant at stage $s$ if $n \leqslant b_{c, s}$ and the hypothesis of $R_{i, n}$ appears to hold at stage $s$. Item (1)(b) in Definition 6.7 implies that there is some $F_{t}$ with $t \geqslant b_{c, s}$ such that we can simultaneously satisfy all permanently relevant $R_{i, n}$ with $(i, n) \in F_{t}$. We guess at such an $F_{t}$, and choose potential witnesses from $\left[b_{c, s}, b_{c+1, s}\right)$ for all relevant $R_{i, n}$ such that $(i, n) \in F_{t}$. We then define $f\left(b_{c+1, s}, s\right)$ to attempt to ensure that these witnesses have the correct type to satisfy these requirements. Even absent interference from other blocks, such an attempt might fail, as defining $f\left(b_{c+1, s}, s\right)$ correctly requires first having an appropriate $t$, then having a type amalgamating $p_{f\left(b_{c+1, s}, s\right)}$ with the various types involved in all the relevant $R_{i, n}$, both of which can be found only by trial and error. (Though once the block stops growing, these will eventually be found.) When such an attempt fails, we need to redefine $b_{c+1, s}$ to create enough room for the next attempt (i.e., we choose it so that there are enough elements below $b_{c+1, s}$ on which $f$ has not yet been defined to provide witnesses for all the $R$-requirements that might be considered in the next attempt at satisfying $\left.S_{b_{c, s}}\right)$. Another issue we need to confront in defining $f\left(b_{c+1, s}, s\right)$ is that $f(k, s-1)$ might have been defined for some $k \in\left(b_{c, s}, b_{c+1, s}\right]$. If so, then we need to use our extension function approximation $g$ to determine $f\left(b_{c+1, s}, s\right)$, as $p_{f\left(b_{c+1, s}, s\right)}$ has to contain $p_{f(k, s-1)}\lceil s-1$ for each such $k$.

In addition, definitions made for two blocks, say the $c$ th and $d$ th for $c<d$, might turn out to be in conflict (i.e., $p_{f\left(b_{c+1, s}, s\right)}$ might turn out not to be a subtype of $\left.p_{f\left(b_{d+1, s}, s\right)}\right)$. Then we injure the $d$ th block, changing the values of $f$ it has defined. When we do so, we increase $b_{e, s}$ for all $e>d$. As we show below, this action ensures that the correctness of our construction can be verified in $\mathrm{RCA}_{0}$.

To each block $c$, we attach parameters $i_{c}$ and $j_{c}$, which might change or be undefined during the construction. These are chosen so that $p_{i_{c}}$ is a type in variables among $x_{0}, \ldots, x_{b_{c+1, s}-1}$ and $\varphi_{j_{c}}\left(x_{0}, \ldots, x_{b_{c+1, s}-1}\right)$ is consistent with $p_{i_{c}}$. We ensure that whenever $i_{c}$ and $j_{c}$ are defined, then so are $i_{d}$ and $j_{d}$ for $d<c$. At the end of each stage $s>0$, we define $f\left(b_{c+1, s}, s\right)=g\left(i_{c}, j_{c}, s\right)$ if these parameters are defined. Thus $i_{c}$ represents a type through which we are attempting to satisfy $S_{b_{c, s}}$, and $j_{c}$ represents a formula that we need to amalgamate with this type to preserve properties (i)-(iii) of $f$. We also assign tuples of witnesses to $R$-requirements corresponding to $S_{b_{c, s}}$, which again might change during the construction. We ensure that if $S_{b_{c, s}}$ has witnesses assigned to it, then $i_{c}$ and $j_{c}$ are defined.

Construction of $f$. At stage 0 , act as follows. Let $b_{j, 0}=j$ for all $j \in \mathbb{N}$. Let $e$ be such that $p_{e}$ is the 0 -type and let $f(0, s)=e$ for all $s \in \mathbb{N}$. For notational convenience, we also let $g\left(i_{-1}, j_{-1}, s\right)$ denote $e$ for all $s \in \mathbb{N}$.

At stage $s>0$, act as follows. Recall that $a_{i}$ is the arity of $p_{i}$. For each $c$ such that $i_{c}$ and $j_{c}$ are defined, say that $(i, n) \in R$ is c-active if $q_{i, n} \uparrow s$ is consistent with $p_{\lim _{u} g\left(i_{c-1}, j_{c-1}, u\right)}$ (which we can check effectively because of the monotonicity of $g$ ). Say that $c$ is satisfied if $i_{c}$ and $j_{c}$ are defined, $s \geqslant b_{c, s-1}$, and there is a $t \in\left[b_{c, s-1}, s\right]$ such that each $c$-active $(i, n) \in F_{t}$ with $n<b_{c, s-1}$ has witnesses 
$j_{0}, \ldots, j_{a_{i}-n-1}$ assigned to it for which

$$
p_{i}\left[x_{0}, \ldots, x_{n-1}, x_{j_{0}}, \ldots, x_{j_{a_{i}-n-1}}\right]\left\lceil s \subset p_{g\left(i_{c}, j_{c}, s\right)} .\right.
$$

Say that $c$ requires attention if $i_{c}$ and $j_{c}$ are defined, $s \geqslant b_{c, s-1}$, and $c$ is not satisfied. If $i_{c}$ and $j_{c}$ are defined, then say they are untenable if $p_{g\left(i_{c}, j_{c}, s\right)} \uparrow s$ is inconsistent with $p_{\lim _{u} g\left(i_{c-1}, j_{c-1}, u\right)}$.

Let $c$ be least such that $i_{c}$ and $j_{c}$ are undefined or untenable, or $c$ requires attention. (Such a $c$ must exist because we define $i_{d}$ and $j_{d}$ for only finitely many $d$ at each stage.) We say that $c$ is active at stage $s$. Let $b_{d, s}=b_{d, s-1}$ for all $d \leqslant c$. Let $k_{s}$ be the number of stages $u<s$ at which $c$ has been active and $b_{c, u}=b_{c, s}$, and let $t=b_{c, s}+n$, where $n$ is such that $k_{s}=\langle n, l\rangle$ for some $l$. Let $b_{c+1, s}$ be large enough so that $\left[b_{c, s}, b_{c+1, s}\right)$ contains at least $\sum_{(i, n) \in F_{t}} a_{i}$ many fresh elements (i.e. ones on which $f$ has never been defined). Let $b_{c+1+m, s}=b_{c+1, s}+m$ for all $m>0$. For $d>c$, undefine $i_{d}, j_{d}$, and all witnesses associated with the $d$ th block.

Say that $R_{i, n}$ is relevant if it is c-active, $n<b_{c, s}$, and $(i, n) \in F_{t}$. Let $R_{l_{0}, n_{0}}, \ldots, R_{l_{r-1}, n_{r-1}}$ be the relevant requirements. For each $i<r$, let $m_{i}=a_{l_{i}}-n_{i}$ and choose fresh distinct elements $w_{0}, \ldots, w_{m_{i}-1} \in\left[b_{c, s}, b_{c+1, s}\right)$, which we call witnesses for $R_{l_{i}, n_{i}}$, so that the tuples of elements chosen for different $i$ are pairwise disjoint. Let $\psi$ be the conjunction of all formulas in $p_{l_{i}}\left[x_{0}, \ldots, x_{n-1}, x_{w_{0}}, \ldots, x_{w_{m_{i}-1}}\right]$ $s$ for $i<r$ and in $p_{g\left(i_{c-1}, j_{c-1}, s\right)} \uparrow s$. (If $r=0$ then $\psi$ is just the propositional constant T.) Let $\theta$ be the conjunction of all formulas appearing in $p_{f(n, u)} \uparrow u$ for $n$ and $u<s$ such that $f(n, u)$ is defined.

All the free variables shared by $\psi$ and $\theta$ are among $x_{0}, \ldots, x_{b_{c, s}-1}$, while $p_{\lim _{u} g\left(i_{c-1}, j_{c-1}, u\right)}$ is a type in the variables $x_{0}, \ldots, x_{b_{c, s}-1}$, so to show that $\psi \wedge$ $\theta$ is consistent, it is enough to show that each of $\psi$ and $\theta$ is consistent with $p_{\lim _{u} g\left(i_{c-1}, j_{c-1}, u\right)}$. That $\psi$ is consistent with $p_{\lim _{u} g\left(i_{c-1}, j_{c-1}, u\right)}$ follows from the definition of a requirement being $c$-active. We show in the verification section below that $\theta$ is also consistent with $p_{\lim _{u} g\left(i_{c-1}, j_{c-1}, u\right)}$, so we assume that this is the case for now. Let $i_{c}$ be least such that $p_{i_{c}}$ is a type in the variables appearing in $\psi$, contains $\psi$, and is consistent with $\theta$. Let $j_{c}$ be such that $\varphi_{j_{c}}$ is the conjunction of $\theta$ with $x_{n}=x_{n}$ for all $n<b_{c+1, s}$. (These tautologies are there to ensure that $\varphi_{j_{c}}$ has the correct arity.)

For each $c \in \mathbb{N}$ such that $i_{c}$ and $j_{c}$ are defined, let $f\left(b_{c+1, s}, s\right)=g\left(i_{c}, j_{c}, s\right)$. For $n \in\left(b_{c, s}, b_{c+1, s}\right)$, if $f\left(b_{c+1, s}, s\right) \neq f\left(b_{c+1, s}, s-1\right)$, then let $f(n, s)$ be such that $p_{f(n, s)}=p_{f\left(b_{c+1, s}, s\right)}\left\lceil x_{0}, \ldots, x_{n-1}\right.$. For all $n<b_{c+1, s}$ such that $f(n, s)$ has not yet been defined, let $f(n, s)=f(n, s-1)$. For all $n>b_{c+1, s}$, let $f(n, s)$ be undefined.

Verification. Having completed the construction of $f$, we verify its correctness. We first need to show that the formula $\theta$ above is in fact consistent with $p_{\lim _{u} g\left(i_{c-1}, j_{c-1}, u\right)}$. By $\left(\Delta_{0^{-}}^{0}\right)$ induction, we may assume that this property holds for all stages prior to $s$. Let $v<s$ be the stage at which $i_{c-1}$ and $j_{c-1}$ were last defined prior to stage $s$. Let $\theta^{\prime}$ be the conjunction of all formulas appearing in $p_{f(n, w) \uparrow w}$ for $n$ and $w \leqslant v$ such that $f(n, w)$ is defined. Then $\varphi_{j_{c-1}}$ is equivalent to $\theta^{\prime}$, so $\theta^{\prime} \in p_{\lim _{u} g\left(i_{c-1}, j_{c-1}, u\right)}$. At each stage $r \in(v, s)$, we maintain the consistency of each $p_{f(n, r)}\left\lceil r\right.$ such that $f(n, r)$ is defined with $p_{\lim _{u} g\left(i_{c-1}, j_{c-1}, u\right)}$, by the definition of untenability and the definition of $f$. Thus $\theta$ is consistent with $p_{\lim _{u} g\left(i_{c-1}, j_{c-1}, u\right)}$.

We now verify that the five properties of $f$ hold. Property (i) is clearly satisfied by the construction. Suppose $f(n, s)$ and $f(n, s+k)$ are both defined and $n \in$ $\left[b_{c, s+k}, b_{c+1, s+k}\right)$. Then either $f(n, s+k)=f(n, s+k-1)$ or $i_{c}$ and $j_{c}$ get defined 
at stage $s+k$, in which case $p_{f\left(b_{c+1, s+k}, s+k\right)}$ is defined so as to contain $p_{f(n, s)} \uparrow s$, and $p_{f(n, s+k)}=p_{f\left(b_{c+1, s+k}, s+k\right)} \uparrow x_{0}, \ldots, x_{n-1}$. Thus property (ii) holds by $\left(\Delta_{0^{-}}^{0}\right.$ )induction on $k$.

Now suppose $f(n, s)$ and $f(n+1, s)$ are both defined. If there is a $c$ such that $n, n+1 \in\left(b_{c, s}, b_{c+1, s}\right]$ then $p_{f(n, s)}=p_{f(n+1, s)}\left\lceil x_{0}, \ldots, x_{n-1}\right.$. Otherwise, there is a $c$ such that $n=b_{c, s}$. If $i_{c}$ and $j_{c}$ do not get redefined at stage $s$ then $c$ is not untenable at stage $s$, so $p_{f\left(b_{c+1, s}, s\right)} \uparrow s=p_{g\left(i_{c}, j_{c}, s\right)} \uparrow s$ is consistent with $\lim _{u} g\left(i_{c-1}, j_{c-1}, u\right)$. Since $p_{f(n, s)} \uparrow s=p_{\lim _{u} g\left(i_{c-1}, j_{c-1}, u\right)} \uparrow s$ and $p_{f(n+1, s)}=p_{f\left(b_{c+1, s}, s\right)} \uparrow x_{0}, \ldots, x_{n}$, we have $p_{f(n, s)} \uparrow s \subseteq p_{f(n+1, s)} \uparrow s$. If $i_{c}$ and $j_{c}$ do get redefined at stage $s$, then $p_{f\left(b_{c+1, s}, s\right)}$ contains $p_{f(n, s)}\left\lceil s=p_{g\left(i_{c-1}, j_{c-1}, s\right)} \uparrow s\right.$, so again $p_{f(n, s)}\left\lceil s \subseteq p_{f(n+1, s)} \uparrow\right.$ $s$. In any case, property (iii) holds.

Thus we are left with verifying that all our requirements are satisfied, so that properties (iv) and (v) hold. Let $b_{c}=\lim _{s} b_{c, s}$, which might be infinite. Suppose that $b_{c}$ is finite. Every time $i_{d}$ and $j_{d}$ are redefined for some $d<c$, the value of $b_{c, s}$ increases, so there is a stage $s_{0}$ by which all these $i_{d}$ and $j_{d}$ have permanent values. In particular, $i_{c-1}$ and $j_{c-1}$ have permanent values (if $c>0$ ). Then there is a stage $s_{1} \geqslant s_{0}$ such that $g\left(i_{c-1}, j_{c-1}, s\right)=g\left(i_{c-1}, j_{c-1}, s_{1}\right)$ for all $s>s_{1}$. Let $h=g\left(i_{c-1}, j_{c-1}, s_{1}\right)$.

Let $t \geqslant b_{c}$ be such that for each $S \subseteq F_{t}$ for which $q_{S}$ is consistent, there is a $p_{j}$ amalgamating all the types in $q_{S}$. Let $B$ be the set of requirements $R_{i, n}$ such that $(i, n) \in F_{t}$ and $n<b_{c}$. Any requirement in $B$ that ceases to be $c$-active after stage $s_{1}$ is never again $c$-active, so $\Sigma_{1}^{0}$-induction suffices to show that there is an $s_{2} \geqslant s_{1}$ such that the collection $R_{l_{0}, n_{0}}, \ldots, R_{l_{m-1}, n_{m-1}}$ of $c$-active requirements in $B$ has stabilized by stage $s_{2}$. By the definition of $c$-active requirement, the types $p_{l_{i}}\left[x_{0}, \ldots, x_{n-1}, y_{i, 0}, \ldots, y_{i, a_{l_{i}}-n_{i}-1}\right]$ for $i<m$ form a consistent set of types, so by the choice of $t$, there is a $p_{j}$ amalgamating them. Again by the definition of $c$ active requirement, $p_{j}$ and $p_{h}$ are consistent, so by pairwise full type amalgamation (which clearly follows from eventual full type amalgamation), there is a type in $X$ amalgamating them. By $\Pi_{1}^{0}$-induction, there is a least $n$ such that $p_{n}$ is a type amalgamating $p_{h}$ and all of the types $p_{l_{i}}\left[x_{0}, \ldots, x_{n-1}, y_{i, 0}, \ldots, y_{i, a_{l_{i}}-n_{i}-1}\right]$ for $i<m$. Then it is easy to check from the definition of $i_{c}$ that there is a stage $s_{3} \geqslant s_{2}$ such that if $i_{c}$ is defined at a stage $s \geqslant s_{3}$ at which $k_{s}$ is of the form $\left\langle t-b_{c}, l\right\rangle$, then $i_{c}=n$.

Say that a stage $s$ is $c$-final if $c$ never requires attention after stage $s$, and $i_{c}$ and $j_{c}$ are never undefined or untenable after stage $s$. It is easy to check that if $i_{c}$ and $j_{c}$ are defined at a stage $s \geqslant s_{3}$ at which $k_{s}$ is of the form $\left\langle t-b_{c}, l\right\rangle$, then $s$ is $c$-final. It is also easy to check that such a definition occurs unless $k_{s}$ never reaches such a value for $s \geqslant s_{3}$. In this case, there must be a $c$-final stage, as $k_{s}$ increases each time $c$ is active. In either case, there is a $c$-final stage $s$. Then $b_{c+1}=b_{c+1, s}$ is finite, and $S_{b_{c}}$ is permanently satisfied by stage $s$. Furthermore, $i_{c}$ and $j_{c}$ have final values at stage $s$, and there is a stage $u>s$ such that $g\left(i_{c}, j_{c}, v\right)=g\left(i_{c}, j_{c}, u\right)$ for all $v>u$. Then $f(n, v)=f(n, u)$ for all $n \in\left(b_{c}, b_{c+1}\right)$, so every $Q_{n}$ in the $c$ th block is satisfied.

Now, given $n \in \mathbb{N}$, let $d$ be least such that $b_{d+1, s} \geqslant n$ for some $s \in \mathbb{N}$, which exists by $\Sigma_{1}^{0}$-induction. Then $b_{d}$ is finite, so there is a final $d$ th block of requirements, and $Q_{n}$ is in that block, and hence is satisfied. Furthermore, $b_{d+1}$ is also finite, so $S_{b_{d+1}}$ is satisfied, and hence so is $S_{n}$, since $n \leqslant b_{d+1}$. 
We now establish two theorems that give us the reversals to $\mathrm{I} \Sigma_{2}^{0}$ of the entries labeled 2 or 3 on our chart.

THEOREM 6.11. The following is provable in $\mathrm{RCA}_{0}$. Let $\varphi$ be a $\Pi_{1}^{0}$ formula defining a total function. For each $n$ and $z$, there is a theory $T$ and a list $X$ of types of $T$ such that the following hold.

(1) X satisfies the pairwise full amalgamation closure conditions.

(2) $X$ has an extension function.

(3) Suppose one of the following conditions holds.

(a) $X$ satisfies the finite free amalgamation closure conditions.

(b) There is a listing of the types of $X$ satisfying the finite full amalgamation closure conditions.

(c) There is a a listing of the types of $X$ satisfying the finite free amalgamation closure conditions, and $\mathrm{B} \Sigma_{2}^{0}$ holds.

Then there is a sequence $\left(m_{0}, \ldots, m_{n-1}\right)$ such that $m_{0}=z$ and $\varphi\left(m_{i}, m_{i+1}\right)$ holds for all $i<n-1$.

Proof. We argue in $\mathrm{RCA}_{0}$. Fix $n$ and $z$. We begin by defining a theory $T$ and a list of types $X$ to handle $3(\mathrm{a})$ and $3(\mathrm{c})$; we then slightly modify them to handle $3(\mathrm{~b})$.

We work in the language with unary relation symbols $P_{i}$ for $i \in \mathbb{N}$, a binary relation symbol $E$, and constant symbols $c_{0}, \ldots, c_{2^{n}-1}$. We begin by defining a set $A$ of axioms. We write some of these informally; formalizing them in our language is straightforward.

A1. $P_{i+1}(x) \rightarrow P_{i}(x)$ for all $i \in \mathbb{N}$.

A2. $\neg P_{0}\left(c_{i}\right)$ for all $i<2^{n}$.

A3. $E$ is an equivalence relation with exactly $2^{n}$ many classes, each containing exactly one $c_{i}$.

A4. Within each equivalence class, for each $i \in \mathbb{N}$ there are infinitely many $x$ such that $P_{i}(x) \wedge \neg P_{i+1}(x)$, and there are infinitely many $x$ such that $\neg P_{0}(x)$.

It is not difficult to show that $A$ is consistent and admits effective quantifier elimination, so that its deductive closure $T$ exists and is a theory. (To conclude that $T$ exists, we also need to know that the set of literal sentences consistent with $A$ exists, as mentioned in the discussion of quantifier elimination at the end of Section 2.2. This is clearly the case, as this set consists exactly of the sentences $\neg P_{k}\left(c_{i}\right)$ for $i<2^{n}$ and $k \in \mathbb{N}$, the sentences $E\left(c_{i}, c_{i}\right)$ for $i<2^{n}$, and the sentences $\neg E\left(c_{i}, c_{j}\right)$ for $i, j<2^{n}$ such that $i \neq j$.)

We begin by showing that $A$ admits effective quantifier elimination, as discussed at the end of Section 2.2. Fix an existential formula $(\exists y) \varphi(\bar{x} y)$, where $\varphi(\bar{x} y)$ is a conjunction of literals. Let $m$ be such that no $P_{i}$ with $i>m$ occurs in $\varphi$, and let $\mathcal{L}^{\prime}$ be the language consisting of $E, c_{0}, \ldots, c_{2^{n}-1}$, and all $P_{i}$ with $i \leqslant m$. As mentioned in Remark 2.3, we may assume that $\varphi$ is an $\mathcal{L}^{\prime}$-atomic diagram that is consistent with the assertion that $E$ is an equivalence relation, and includes $\neg u=v$ for all $u$ such that $u \in \bar{x}$ or $u$ is a constant symbol, and all $v \in \bar{x} y$ distinct from $u$.

Suppose that at least one of the following conditions holds. 
(1) Both $P_{i}(u)$ and $\neg P_{j}(u)$ for $j<i$ occur in $\varphi$, where $u$ is a variable or constant symbol.

(2) $P_{0}\left(c_{i}\right)$ occurs in $\varphi$ for some $i<2^{n}$.

(3) $E\left(c_{i}, c_{j}\right)$ for $i \neq j$ occurs in $\varphi$.

(4) There is a $u \in \bar{x} y$ such that $\neg E\left(u, c_{i}\right)$ occurs in $\varphi$ for all $i<2^{n}$.

Then $A \vdash(\exists y) \psi(\bar{x} y) \leftrightarrow \mathrm{F}$. Otherwise, the fourth axiom family of $A$ implies that $A \vdash(\exists y) \varphi(\bar{x} y) \leftrightarrow \theta(\bar{x})$, where $\theta$ is the result of removing from $\varphi$ all literals involving $y$ (and hence is just $\mathrm{T}$ if all literals in $\varphi$ involve $y$ ).

To show that $T$ is consistent, we provide a model $\mathcal{M}$ of $A$. By effective quantifier elimination, it suffices to specify the atomic diagram of $\mathcal{M}$. Let the domain of $\mathcal{M}$ consist of all numbers of the form $\langle i, j, k\rangle$, where $i<2^{n}$. Let $c_{i}^{\mathcal{M}}=\langle i, 0,0\rangle$. Let $E^{\mathcal{M}}(x, y)$ hold if and only if $x$ and $y$ have the same first coordinate, and let $P_{m}^{\mathcal{M}}(x)$ hold if and only if $x=\langle i, j, k\rangle$ and $m<j$. It is easy to check that $\mathcal{M}$ is a model of A.

We now define our list $X$ of types of $T$, which satisfies the pairwise full amalgamation closure conditions and has an extension function. By effective quantifier elimination, we can identify types of $T$ with atomic types. Notice that the 0 -type $T$ corresponds to the set of literal sentences consistent with $A$ discussed above, which we refer to as the 0-type. From now on, we refer to an atomic type as simply a type.

We begin by naming the types of $T$. Each 1-type in a given variable $x$ includes the 0 -type, as well as $x=x$ and $E(x, x)$. Thus, the 1-types of $T$ in the variable $x$ can be listed as follows.

(1) $s_{i}(x)$ for $i<2^{n}$, which includes $x=c_{i}, c_{i}=x, E\left(x, c_{i}\right)$, and $E\left(c_{i}, x\right)$, as well as $\neg x=c_{j}, \neg c_{j}=x, \neg E\left(x, c_{j}\right)$, and $\neg E\left(c_{j}, x\right)$ for $j \neq i$, and $\neg P_{m}(x)$ for all $m \in \mathbb{N}$.

(2) $q_{i, k}(x)$ for $i<2^{n}$ and $k \in \mathbb{N}$, which includes $\neg x=c_{i}, \neg c_{i}=x, E\left(x, c_{i}\right)$, and $E\left(c_{i}, x\right)$, as well as $\neg x=c_{j}, \neg c_{j}=x, \neg E\left(x, c_{j}\right)$, and $\neg E\left(c_{j}, x\right)$ for $j \neq i$, and also $P_{m}(x)$ for $m<k$ and $\neg P_{m}(x)$ for $m \geqslant k$.

(3) $q_{i, \infty}(x)$ for $i<2^{n}$, which includes $\neg x=c_{i}, \neg c_{i}=x, E\left(x, c_{i}\right)$, and $E\left(c_{i}, x\right)$, as well as $\neg x=c_{j}, \neg c_{j}=x, \neg E\left(x, c_{j}\right)$, and $\neg E\left(c_{j}, x\right)$ for $j \neq i$, and also $P_{m}(x)$ for all $m \in \mathbb{N}$.

Now each $k$-type of $T$ in a tuple of variables $\left(x_{0}, \ldots, x_{k-1}\right)$ can be specified by two items: a list of 1-types $t=\left(t_{0}, \ldots, t_{k-1}\right)$ (i.e., each $t_{j}$ is of the form $s_{i}, q_{i, l}$, or $\left.q_{i, \infty}\right)$ and an equivalence relation $D$ on $[0, k)$ such that if $D(i, j)$ then $t_{i}=t_{j}$, and if $t_{i}=t_{j}=s_{l}$ for some $l$, then $D(i, j)$. (We say that $D$ is compatible with $t$.) This type, which we denote by $r_{t, D}\left(x_{0}, \ldots, x_{k-1}\right)$, is specified as follows.

(1) $r_{t, D}$ includes the 0-type, and for each $i<k$, it includes $t_{i}\left(x_{i}\right)$.

(2) If $t_{i}$ and $t_{j}$ are both among $s_{m}, q_{m, l}$, and $q_{m, \infty}$ for the same $m$, then $r_{t, D}$ includes $E\left(x_{i}, x_{j}\right)$. Otherwise it includes $\neg E\left(x_{i}, x_{j}\right)$.

(3) If $D(i, j)$ then $r_{t, D}$ includes $x_{i}=x_{j}$. Otherwise it includes $\neg x_{i}=x_{j}$.

Let $s=\left(m_{0}, \ldots, m_{l-1}\right)$ be a sequence of natural numbers. If $\varphi\left(m_{i}, m_{i+1}\right)$ holds for all $i<l-1$ (where $\varphi$ is as in the statement of the theorem), then let $N_{s}=\infty$. Otherwise, let $N_{s}$ be the least stage greater than $l$ at which we find that this condition does not hold. Let $r_{t, D}^{s}$ be type obtained from $r_{t, D}$ by replacing each $t_{i}$ of the form $q_{j, l^{\prime}}$ such that $l^{\prime}>N_{s}$ or $l^{\prime}=\infty$ by $q_{j, N_{s}}$. Note that $r_{t, D}^{s}$ is obtained 
effectively from $r_{t, D}$ and $s$. (Note also that we are not claiming that the function $s \mapsto N_{s}$ exists.)

For a type $r_{t, D}$ of $T$ with $t=\left(t_{0}, \ldots, t_{k-1}\right)$, let $b_{t, D}$ be the number of $i<k$ such that $t_{i}$ is of the form $q_{l, \infty}$ for some $l$ and $\neg D(i, j)$ for all $j<i$. Let $X$ be an effective listing of all types $r_{t, D}^{s}(\bar{x})$, where

(1) $t$ ranges over all lists of 1-types,

(2) $D$ ranges over all equivalence relations compatible with $t$,

(3) $s$ ranges over all sequences with first element $z$ such that $|s| \geqslant \log _{2} b_{t, D}$, and

(4) $\bar{x}$ ranges over all tuples of variables of length $|t|$.

(The use of the logarithm here might seem mysterious, but is important in showing that $X$ is closed under pairwise full type amalgamation.) We verify that $X$ satisfies the pairwise full amalgamation closure conditions and has an extension function.

The 0-type is in $X$, and $X$ is clearly closed under variable substitutions. Closure under taking subtypes is straightforward to verify. We now verify that $X$ satisfies the extension condition, and in fact has an extension function.

Fix $r_{t, D}^{s}\left(x_{0}, \ldots, x_{|t|-1}\right) \in X$ and a $\psi\left(x_{0}, \ldots, x_{|t|-1}, x_{|t|}, \ldots, x_{k-1}\right)$ consistent with this type. By effective quantifier elimination (and the fact that if a type includes a formula, then it includes any disjunction involving that formula), we may assume that $\psi$ is a conjunction of literals. We may also assume that if $\psi$ contains $u=v$, then it contains $v=u$, and similarly with other literals involving equality and $E$.

We first extend $D$ to $i$ with $|t| \leqslant i<k$ as follows. If $\psi$ includes $x_{i}=x_{j}$, or both $x_{i}=c_{m}$ and $x_{j}=c_{m}$, then let $D(i, j)$ hold. If $\psi$ includes $x_{i}=c_{m}$ and $t_{j}=s_{m}$ for some $j<|t|$, then let $D(i, j)$ hold. Then close $D$ to make it an equivalence relation.

Now let $d$ be least such that no $P_{i}$ appears in $\psi$ for $i>d$. We can check effectively whether $N_{s}>d$. If so, then proceed as follows. First define a sequence $t^{\prime}=\left(t_{0}^{\prime}, \ldots, t_{k-1}^{\prime}\right)$. For $i<|t|$, let $t_{i}^{\prime}=t_{i}$. Now proceed by recursion starting with $i=|t|$. If $\psi$ includes $x_{i}=x_{j}$ for $j<i$ then let $t_{i}^{\prime}=t_{j}^{\prime}$, and if $\psi$ includes $x_{i}=c_{m}$ then let $t_{i}^{\prime}=s_{m}$. Otherwise, let $m$ be such that $\psi$ includes $E\left(x_{i}, c_{m}\right)$, if there is such an $m$, and otherwise let $m$ be least such that $\psi$ does not include $\neg E\left(x_{i}, c_{m}\right)$. Let $l$ be the largest number such that $\psi$ includes $P_{l}\left(x_{i}\right)$, or -1 if there is no such number. Let $t_{i}^{\prime}=q_{m, l+1}$. Now it is easy to check that $r_{t^{\prime}, D}^{s}$ amalgamates $r_{t, D}^{s}$ and $\psi$.

Now suppose that $N_{s} \leqslant d$. Then we can effectively find a $u$ such that $r_{u, D}=$ $r_{t, D}^{s}$. Define $t^{\prime}$ as in the previous paragraph, but using $u$ in place of $t$. Let $s^{\prime}$ be a sequence of natural numbers starting with $z$ with length greater than $d$. Then it is easy to check that $r_{t^{\prime}, D}^{s^{\prime}}$ amalgamates $r_{t, D}^{s}$ and $\psi$.

Thus $X$ satisfies the extension condition. Since the above procedure for obtaining extensions is effective, $X$ in fact has an extension function.

Finally, we verify that $X$ satisfies the pairwise full type amalgamation condition. Let $r_{t, D}^{s}(\bar{x} \bar{y}), r_{t^{\prime}, D^{\prime}}^{s^{\prime}}(\bar{x} \bar{z}) \in X$ be basically consistent, where $\bar{y}$ and $\bar{z}$ are disjoint. If $N_{s} \in \mathbb{N}$ then $r_{t, D}^{s}$ is a principal type, so the existence of a type amalgamating $r_{t, D}^{s}$ and $r_{t^{\prime}, D^{\prime}}^{s^{\prime}}$ follows from the fact that $X$ satisfies the extension condition. The same is true if $N_{s^{\prime}} \in \mathbb{N}$, so we may assume that $N_{s}=N_{s^{\prime}}=\infty$. Without loss of generality, assume that $|s| \geqslant\left|s^{\prime}\right|$. By the hypothesis on $\varphi$, we can extend $s$ by one element to obtain a sequence $\widehat{s}$ of length $|s|+1$ such that $N_{\widehat{s}}=\infty$. Since $D$ and $D^{\prime}$ must be equal on the variables in $\bar{x}$, there is a minimal equivalence relation $\widehat{D}$ 
on the variables $\bar{x} \bar{y} \bar{z}$ extending $D \cup D^{\prime}$. Since $t$ and $t^{\prime}$ agree on the variables in $\bar{x}$, there is a $\widehat{t}$ that agrees with $t$ on the variables in $\bar{x} \bar{y}$ and with $t^{\prime}$ on the variables in $\bar{x} \bar{z}$. Then $b_{\widehat{t}, \widehat{D}} \leqslant b_{t, D}+b_{t^{\prime}, D^{\prime}}$, and hence

$$
|\widehat{s}|=|s|+1 \geqslant \max \left(\log _{2} b_{t, d}, \log _{2} b_{t^{\prime}, d^{\prime}}\right)+1 \geqslant \log _{2}\left(b_{t, D}+b_{t^{\prime}, D^{\prime}}\right) \geqslant \log _{2} b_{\widehat{t}, \widehat{D}},
$$

so $r_{\widehat{t}, \widehat{D}}^{\widehat{s}}(\bar{x} \bar{y} \bar{z})$ is in $X$ and amalgamates $r_{t, D}^{s}(\bar{x} \bar{y})$ and $r_{t^{\prime}, D^{\prime}}^{s^{\prime}}(\bar{x} \bar{z})$.

We are now ready to prove the theorem in cases $3(\mathrm{a})$ and $3(\mathrm{c})$.

For 3(a), suppose that $X$ satisfies the finite free amalgamation closure conditions, and let $\left(x_{0}, \ldots, x_{2^{n}-1}\right)$ be a tuple of variables. The sequence of types $q_{0, \infty}\left(x_{0}\right), \ldots, q_{2^{n}-1, \infty}\left(x_{2^{n}-1}\right)$ is in $X$, so there is a type $r_{t, D}^{s}\left(x_{0}, \ldots, x_{2^{n}-1}\right)$ in $X$ amalgamating these types. We have $\log _{2} b_{t, D}=n$, so the sequence $s=(z=$ $\left.m_{0}, \ldots, m_{l-1}\right)$ has length at least $n$, and $\varphi\left(m_{i}, m_{i+1}\right)$ for all $i<l-1$.

For 3(c), suppose there is a listing $Y=\left\{h_{i}\right\}_{i \in \mathbb{N}}$ of the types of $X$ satisfying the finite free amalgamation closure conditions, and $\mathrm{B} \Sigma_{2}^{0}$ holds. Let $\left(x_{0}, \ldots, x_{2^{n}-1}\right)$ be a tuple of variables. Each $q_{i, \infty}\left(x_{i}\right)$ is in $Y$, and the property $P(i, j)$ that holds if and only if $h_{j}=q_{i, \infty}\left(x_{i}\right)$ is $\Pi_{1}^{0}$, so by the finite axiom of choice for $\Pi_{1}^{0}$ properties, the whole sequence of types $q_{0, \infty}\left(x_{0}\right), \ldots, q_{2^{n}-1, \infty}\left(x_{2^{n}-1}\right)$ is in $Y$. So $Y$ has a type amalgamating these types, and hence so does $X$. Now the argument is as in the previous paragraph.

For 3(b), we modify our example by removing the constants. That is, our language now has only the $P_{i}$ and $E$, and our axioms consist of A1, the first part of A3, and A4. Effective quantifier elimination and consistency follow by arguments much like the ones above. Our theory $T$ now has only two kinds of 1-types, $q_{k}(x)$, which includes $P_{m}(x)$ for $m<k$ and $\neg P_{m}(x)$ for $m \geqslant k$; and $q_{\infty}(x)$, which includes $P_{m}(x)$ for all $m \in \mathbb{N}$.

Our $k$-types are now of the form $r_{t, D, F}$, where $t$ and $D$ are as before and $F$ is an equivalence relation on $[0,|t|)$ such that if $D(i, j)$ then $F(i, j)$. The type $r_{t, D, F}\left(x_{0}, \ldots, x_{k-1}\right)$ is specified as follows.

(1) $r_{t, D, F}$ includes the 0-type, and for each $i<|t|$, it includes $t_{i}\left(x_{i}\right)$.

(2) If $F(i, j)$ then $r_{t, D, F}$ includes $E\left(x_{i}, x_{j}\right)$. Otherwise it includes $\neg E\left(x_{i}, x_{j}\right)$.

(3) If $D(i, j)$ then $r_{t, D, F}$ includes $x_{i}=x_{j}$. Otherwise it includes $\neg x_{i}=x_{j}$.

The types $r_{t, D, F}^{s}$ and the list of types $X$ are defined much as before. The proof that $X$ is closed under the pairwise full amalgamation closure conditions and has an extension function is also a straightforward modification of the previous case.

Now suppose there is a listing of the types in $X$ satisfying the finite full amalgamation closure conditions. By Proposition 6.6, this listing has an extension function approximation. By Theorem 6.10 , there is a homogeneous model $\mathcal{A}$ such that $X$ is an enumeration of $\mathbb{T}(\mathcal{A})$. Let $c$ be an element of $\mathcal{A}$ satisfying $q_{\infty}$, which must exists since $q_{\infty} \in X$, and let $a$ be an element of the same $E^{\mathcal{A}}$ equivalence class as $c$ such that $\neg P_{0}^{\mathcal{A}}(a)$. Let $b_{0}, \ldots, b_{2^{n}-1}$ be a sequence consisting of representatives of each of the $E^{\mathcal{A}}$ equivalence classes, such that $\neg P_{0}^{\mathcal{A}}\left(b_{i}\right)$ for all $i<2^{n}$. Then $a \equiv b_{i}$ for each $i<2^{n}$, so, by homogeneity, there are $d_{0}, \ldots, d_{2^{n}-1}$ such that, for each $i<2^{n}$, we have $a c \equiv b_{i} d_{i}$, which implies that $d_{i}$ is in the same $E^{\mathcal{A}}$ equivalence class as $b_{i}$ and has type $q_{\infty}$. The type of $\left(d_{0}, \ldots, d_{2^{n}-1}\right)$ is in $X$, and is of the form $r_{t, D, F}^{s}(\bar{x})$. Then $s=\left(z=m_{0}, \ldots, m_{l-1}\right)$ has length at least $n$, and $\varphi\left(m_{i}, m_{i+1}\right)$ holds for all $i<l-1$. 
THEOREM 6.12. The following is provable in $\mathrm{RCA}_{0}$. Let $\varphi$ be a $\Pi_{1}^{0}$ formula defining a total function. For each $n$ and $z$, there is a theory $T$ and a list $X$ of types of $T$ such that the following hold.

(1) X satisfies the 1-point full amalgamation closure conditions.

(2) $X$ has an extension function.

(3) If $X$ satisfies the pairwise free amalgamation closure conditions, then there is a sequence $\left(m_{0}, m_{1}, \ldots, m_{n-1}\right)$ such that $m_{0}=z$ and $\varphi\left(m_{i}, m_{i+1}\right)$ holds for all $i<n-1$.

Proof. We argue in $\mathrm{RCA}_{0}$. Fix $n$ and $z$. Assume without loss of generality that $n$ is even. We use the same theory $T$ as in the proof of Theorem 6.11 (in its original version, with constants), except that we now have $n$ many equivalence classes, rather than $2^{n}$ many. We also adopt the notation of that proof for naming our types, except that $r_{t, D}^{s}$ is now defined as the type obtained from $r_{t, D}$ by replacing each $t_{i}$ of the form $q_{j, l}$ such that $l>N_{s}$ or $l=\infty$ by $q_{j, N_{s}}$, as long as there is no $j<\frac{n}{2}$ for which $D(i, j)$ holds. Thus, the difference between this definition and the old one is that we do not alter the 1-types of the first $\frac{n}{2}$ many variables in $r_{t, D}$ (or any other variable that $r_{t, D}$ declares to be equal to one of the first $\frac{n}{2}$ many variables). The effect of this change is that our list $X$ now includes all $\frac{n}{2}$-types of $T$ but not all $n$-types unless a sequence $m_{0}, \ldots, m_{n-1}$ as above exists.

Let $X$ be an effective listing of all types $r_{t, D}^{s}(\bar{x})$, where

(1) $t$ ranges over all lists of 1-types,

(2) $D$ ranges over all equivalence relations compatible with $t$,

(3) $s$ ranges over all sequences with first element $z$ such that $|s| \geqslant b_{t, D}$ (where $b_{t, D}$ is as in the proof of Theorem 6.11), and

(4) $\bar{x}$ ranges over all tuples of variables of length $|t|$.

As before, the 0-type is in $X$, and $X$ is closed under variable substitutions and taking subtypes. The proof that $X$ satisfies the extension condition, and in fact has an extension function, is also as before.

To verify that $X$ satisfies the 1-point full type amalgamation condition, let $r_{t, D}^{s}(\bar{x} y), r_{t^{\prime}, D^{\prime}}^{s^{\prime}}(\bar{x} z) \in X$ be basically consistent, where $y$ and $z$ are different variables. As before, we may assume that $N_{s}=N_{s^{\prime}}=\infty$, as otherwise the existence of an amalgamator for these types follows from the fact that $X$ satisfies the extension condition. Without loss of generality, assume that $|s| \geqslant\left|s^{\prime}\right|$. By the hypothesis on $\varphi$, we can extend $s$ by one element to obtain a sequence $\widehat{s}$ of length $|s|+1$ such that $N_{\widehat{s}}=\infty$. Since $D$ and $D^{\prime}$ must be equal on the variables in $\bar{x}$, there is a minimal equivalence relation $\widehat{D}$ on the variables $\bar{x} y z$ extending $D \cup D^{\prime}$. Since $t$ and $t^{\prime}$ agree on the variables in $\bar{x}$, there is a $\widehat{t}$ that agrees with $t$ on the variables in $\bar{x} y$ and with $t^{\prime}$ on the variables in $\bar{x} z$. Then $b_{\widehat{t}, \widehat{D}} \leqslant b_{t, D}+1$, so $r_{\widehat{t}, \widehat{D}}^{\widehat{s}}(\bar{x} y z)$ is in $X$ and amalgamates $r_{t, D}^{s}(\bar{x} y)$ and $r_{t^{\prime}, D^{\prime}}^{s^{\prime}}(\bar{x} z)$.

Now suppose that $X$ satisfies the pairwise free type amalgamation condition, and let $\left(x_{0}, \ldots, x_{n-1}\right)$ be a tuple of variables. Let $t=\left(q_{0, \infty}, \ldots, q_{\frac{n}{2}-1, \infty}\right)$, let $D=$ $\left\{(i, i): i<\frac{n}{2}\right\}$, let $t^{\prime}=\left(q_{\frac{n}{2}, \infty}, \ldots, q_{n-1, \infty}\right)$, and let $D^{\prime}=\left\{(i, i): \frac{n}{2} \leqslant i<n\right\}$. Then $r_{t, D}^{s}=r_{t, D}$ and $r_{t^{\prime}, D^{\prime}}^{s}=r_{t^{\prime}, D^{\prime}}$ for all $s$, so $r_{t, D}\left(x_{0}, \ldots, x_{\frac{n}{2}-1}\right), r_{t^{\prime}, D^{\prime}}\left(x_{\frac{n}{2}}, \ldots, x_{n-1}\right) \in$ $X$. Thus, there is a type $r_{\widehat{t}, \widehat{D}}^{s}\left(x_{0}, \ldots, x_{n-1}\right) \in X$ amalgamating them. Then $s=$ $\left(z=m_{0}, \ldots, m_{l-1}\right)$ has length at least $n$, and $\varphi\left(m_{i}, m_{i+1}\right)$ for all $i<l-1$.

We can now give our justifications of the correctness of Table 6.1. 
TABLE 6.3. Versions of Theorem 6.4

\begin{tabular}{|c|c|c|c|c|c|}
\hline & $\begin{array}{c}\text { p'wise } \\
\text { free } \\
\text { amalg. }\end{array}$ & $\begin{array}{c}\text { finite } \\
\text { free } \\
\text { amalg. }\end{array}$ & $\begin{array}{c}\text { 1-pt. } \\
\text { full } \\
\text { amalg. }\end{array}$ & $\begin{array}{c}\text { p'wise } \\
\text { full } \\
\text { amalg. }\end{array}$ & $\begin{array}{c}\text { finite } \\
\text { full } \\
\text { amalg. }\end{array}$ \\
\hline$X$ has pairwise amalg. & $\sqrt{ }$ & $\sqrt{ }$ & 2 & $\sqrt{ }$ & $\sqrt{ }$ \\
\hline$X$ has finite amalg. & 2 & $\sqrt{ }$ & 2 & 2 & $\sqrt{ }$ \\
\hline$\exists$ listing w/ finite amalg. & 3 & $\sqrt{ }$ & 2 & 2 & $\sqrt{ }$ \\
\hline$\exists$ model & 3 & 1 & 2 & 3 & 1 \\
\hline$\exists$ 1-pt. homogeneous model & $\mathrm{X}$ & $\mathrm{X}$ & 2 & 3 & 1 \\
\hline$\exists$ 1-homogeneous model & $\mathrm{X}$ & $\mathrm{X}$ & 2 & 3 & 1 \\
\hline$\exists$ homogeneous model & $\mathrm{X}$ & $\mathrm{X}$ & 2 & 2 & 1 \\
\hline$\exists$ listing w/ EF & 4 & 1 & 5 & 4 & 1 \\
\hline$\exists$ listing w/ EF + p'wise am. & 4 & 1 & 2 & 4 & 1 \\
\hline$\exists$ listing w/ EF + fin. am. & 3 & 1 & 2 & 2 & 1 \\
\hline
\end{tabular}

TheOREm 6.13. The implications described in Table 6.1 hold.

Proof. We begin by repeating Table 6.1 as Table 6.3 , and recalling its key for convenience.

- A 1 means that the principle is true in $\mathrm{RCA}_{0}$.

- A 2 means that the principle is equivalent to $\mathrm{I}_{2}^{0}$ over $\mathrm{RCA}_{0}$.

- A 3 means that the principle is provable from $\Pi_{1}^{0} \mathrm{GA}$ over $\mathrm{RCA}_{0}$ and is equivalent to $\mathrm{I} \Sigma_{2}^{0}$ over $\mathrm{RCA}_{0}+\mathrm{B} \Sigma_{2}^{0}$ (and hence not provable in $\mathrm{RCA}_{0}$, or even $\left.\mathrm{RCA}_{0}+\mathrm{B} \Sigma_{2}^{0}\right)$.

- A 4 means that the principle is provable from $\Pi_{1}^{0} \mathrm{GA}$ over $\mathrm{RCA}_{0}$, but its strength is otherwise unknown.

- A 5 means that the principle is provable from $\mathrm{I}_{2}^{0}$ over $\mathrm{RCA}_{0}$, but its strength is otherwise unknown.

We now arrange our justifications into two charts, the first showing in which systems our principles are provable, and the second showing what they imply.

A: Provable in $\mathrm{RCA}_{0}$ by part (1) of Theorem 6.10.

B: Provable in $\mathrm{RCA}_{0}$ by part (1) of Theorem 6.10 and Proposition 6.6.

C: Provable in $\mathrm{RCA}_{0}$ by part (2) of Theorem 6.10 .

D: Provable in $\mathrm{RCA}_{0}$ by part (2) of Theorem 6.10 and Proposition 6.6.

E: Provable in $\mathrm{RCA}_{0}+\mathrm{I} \Sigma_{2}^{0}$ by Theorem 5.11 .

F: Provable in $\mathrm{RCA}_{0}+\mathrm{I} \Sigma_{2}^{0}$ by part (1) of Theorem 6.10 and Theorem 5.11. (We do not really need Theorem 6.10 here, since the finite injury construction in the usual proof of Theorem 6.4 can be carried out in $\mathrm{RCA}_{0}+\mathrm{I} \Sigma_{2}^{0}$.)

G: Provable in $\mathrm{RCA}_{0}+\mathrm{I} \Sigma_{2}^{0}$ by part (1) of Theorem 6.10, Proposition 6.6, and Theorem 5.11. (As above, we do not really need Theorem 6.10 here.)

$\mathrm{H}$ : Provable in $\mathrm{RCA}_{0}+\Pi_{1}^{0} \mathrm{GA}$ by part (3) of Theorem 6.10 .

I: Provable in $\mathrm{RCA}_{0}+\Pi_{1}^{0} \mathrm{GA}$ by part (3) of Theorem 6.10 and Proposition 6.6 .

J: Provable in $\mathrm{RCA}_{0}+\Pi_{1}^{0} \mathrm{GA}$ by part (4) of Theorem 6.10 .

$\mathrm{K}$ : Provable in $\mathrm{RCA}_{0}+\Pi_{1}^{0} \mathrm{GA}$ by part (4) of Theorem 6.10 and Proposition 5.13. 
TABLE 6.4. Justifications of Table 6.1: where provable

\begin{tabular}{|c|c|c|c|c|c|}
\hline & $\begin{array}{c}\text { p'wise } \\
\text { free } \\
\text { amalg. }\end{array}$ & $\begin{array}{c}\text { finite } \\
\text { free } \\
\text { amalg. }\end{array}$ & $\begin{array}{c}\text { 1-pt. } \\
\text { full } \\
\text { amalg. }\end{array}$ & $\begin{array}{c}\text { p'wise } \\
\text { full } \\
\text { amalg. }\end{array}$ & $\begin{array}{c}\text { finite } \\
\text { full } \\
\text { amalg. }\end{array}$ \\
\hline$X$ has pairwise amalg. & $\sqrt{ }$ & $\sqrt{ }$ & $2 \mathrm{E}$ & $\sqrt{ }$ & $\sqrt{ }$ \\
\hline$X$ has finite amalg. & $2 \mathrm{E}$ & $\sqrt{ }$ & $2 \mathrm{E}$ & $2 \mathrm{E}$ & $\sqrt{ }$ \\
\hline$\exists$ listing w/ finite amalg. & $3 \mathrm{~K}$ & $\sqrt{ }$ & $2 \mathrm{E}$ & $2 \mathrm{E}$ & $\sqrt{ }$ \\
\hline$\exists$ model & $3 \mathrm{~J}$ & $1 \mathrm{C}$ & $2 \mathrm{~F}$ & $3 \mathrm{H}$ & $1 \mathrm{~A}$ \\
\hline$\exists$ 1-pt. homogeneous model & $\mathrm{X}$ & $\mathrm{X}$ & $2 \mathrm{~F}$ & $3 \mathrm{H}$ & $1 \mathrm{~A}$ \\
\hline$\exists$ 1-homogeneous model & $\mathrm{X}$ & $\mathrm{X}$ & $2 \mathrm{~F}$ & $3 \mathrm{H}$ & $1 \mathrm{~A}$ \\
\hline$\exists$ homogeneous model & $\mathrm{X}$ & $\mathrm{X}$ & $2 \mathrm{~F}$ & $2 \mathrm{~F}$ & $1 \mathrm{~A}$ \\
\hline$\exists$ listing w/ EF & $4 \mathrm{~L}$ & $1 \mathrm{D}$ & $5 \mathrm{G}$ & $4 \mathrm{I}$ & $1 \mathrm{~B}$ \\
\hline$\exists$ listing w/ EF + p'wise am. & $4 \mathrm{~L}$ & $1 \mathrm{D}$ & $2 \mathrm{G}$ & $4 \mathrm{I}$ & $1 \mathrm{~B}$ \\
\hline$\exists$ listing w/ EF + fin. am. & $3 \mathrm{~L}$ & $1 \mathrm{D}$ & $2 \mathrm{G}$ & $2 \mathrm{G}$ & $1 \mathrm{~B}$ \\
\hline
\end{tabular}

TABLE 6.5. Justifications of Table 6.1: implications

\begin{tabular}{|c|c|c|c|c|c|}
\hline & $\begin{array}{c}\text { p'wise } \\
\text { free } \\
\text { amalg. }\end{array}$ & $\begin{array}{c}\text { finite } \\
\text { free } \\
\text { amalg. }\end{array}$ & $\begin{array}{c}\text { 1-pt. } \\
\text { full } \\
\text { amalg. }\end{array}$ & $\begin{array}{c}\text { p'wise } \\
\text { full } \\
\text { amalg. }\end{array}$ & $\begin{array}{c}\text { finite } \\
\text { full } \\
\text { amalg. }\end{array}$ \\
\hline$X$ has pairwise amalg. & $\sqrt{ }$ & $\sqrt{ }$ & $2 \mathrm{P}$ & $\sqrt{ }$ & $\sqrt{ }$ \\
\hline$X$ has finite amalg. & $2 \mathrm{M}$ & $\sqrt{ }$ & $2 \mathrm{~N}$ & $2 \mathrm{~N}$ & $\sqrt{ }$ \\
\hline$\exists$ listing w/ finite amalg. & $3 \mathrm{~S}$ & $\sqrt{ }$ & $2 \mathrm{~N}$ & $2 \mathrm{~N}$ & $\sqrt{ }$ \\
\hline$\exists$ model & $3 \mathrm{~T}$ & 1 & $2 \mathrm{R}$ & $3 \mathrm{~T}$ & 1 \\
\hline$\exists$ 1-pt. homogeneous model & $\mathrm{X}$ & $\mathrm{X}$ & $2 \mathrm{R}$ & $3 \mathrm{~T}$ & 1 \\
\hline$\exists$ 1-homogeneous model & $\mathrm{X}$ & $\mathrm{X}$ & $2 \mathrm{R}$ & $3 \mathrm{~T}$ & 1 \\
\hline$\exists$ homogeneous model & $\mathrm{X}$ & $\mathrm{X}$ & $2 \mathrm{R}$ & $2 \mathrm{O}$ & 1 \\
\hline$\exists$ listing w/ EF & 4 & 1 & 5 & 4 & 1 \\
\hline$\exists$ listing w/ EF + p'wise am. & 4 & 1 & $2 \mathrm{Q}$ & 4 & 1 \\
\hline$\exists$ listing w/ EF + fin. am. & $3 \mathrm{~S}$ & 1 & $2 \mathrm{~N}$ & $2 \mathrm{~N}$ & 1 \\
\hline
\end{tabular}

L: Provable in $\mathrm{RCA}_{0}+\Pi_{1}^{0} \mathrm{GA}$ by part (4) of Theorem 6.10 and Proposition 6.6.

M: Implies $\mathrm{I}_{2}^{0}$ over $\mathrm{RCA}_{0}$ by Theorem 6.11 , applied with hypothesis $(3)(\mathrm{a})$. $\mathrm{N}$ : Implies $\mathrm{I} \Sigma_{2}^{0}$ over $\mathrm{RCA}_{0}$ by Theorem 6.11 , applied with hypothesis $(3)(\mathrm{b})$.

O: Implies $\mathrm{I} \Sigma_{2}^{0}$ over $\mathrm{RCA}_{0}$ by Theorem 6.11 , applied with hypothesis $(3)(\mathrm{b})$, and Proposition 5.13.

P: Implies $\mathrm{I} \Sigma_{2}^{0}$ over $\mathrm{RCA}_{0}$ by Theorem 6.12 .

Q: Implies $\mathrm{I} \Sigma_{2}^{0}$ over $\mathrm{RCA}_{0}$ by Theorem 6.12 and Proposition 5.10.

$\mathrm{R}$ : Implies $\mathrm{I} \Sigma_{2}^{0}$ over $\mathrm{RCA}_{0}$ by Theorem 6.12 and Propositions 5.10 and 5.13.

$\mathrm{S}$ : Implies $\mathrm{I} \Sigma_{2}^{0}$ over $\mathrm{RCA}_{0}+\mathrm{B} \Sigma_{2}^{0}$ by Theorem 6.11 , applied with hypothesis (3) (c).

$\mathrm{T}$ : Implies $\mathrm{I} \Sigma_{2}^{0}$ over $\mathrm{RCA}_{0}+\mathrm{B}_{2}^{0}$ by Theorem 6.11 , applied with hypothesis (3)(c), and Proposition 5.13. 



\section{CHAPTER 7}

\section{The Reverse Mathematics of Model Existence Theorems}

In this chapter, we compare the principles discussed in Chapter 3 and Section 5.5. We can think of each of HMT, 1-HMT, 1-PHMT, WMT, and SMT as the combination of a principle asserting the existence of the desired model given the existence of an extension function approximation, and a principle asserting the existence of an extension function approximation given the stated hypothesis. The first kind of principle was analyzed in the previous chapter. We now examine the latter. For a set $\mathcal{C}$ of closure conditions, define the following principle.

EFAE $_{\mathcal{C}}$ (Extension Function Approximation Existence Under the Closure Conditions $\mathcal{C}$ ): Every list of types satisfying the closure conditions $\mathcal{C}$ has an extension function approximation.

Of course, for this principle to hold, $\mathcal{C}$ must contain at least the extension condition, i.e., item (4) in Theorems 5.1 and 5.7.

The five sets of closure conditions considered above are the pairwise free, finite free, 1-point full, pairwise full, and finite full amalgamation closure conditions, which correspond to SMT, WMT, 1-PHMT, 1-HMT, and HMT, respectively.

We show in Theorem 7.5 that if $\mathcal{C}$ denotes the finite full or finite free amalgamation closure conditions, then $\mathrm{EFAE}_{\mathcal{C}}$ is equivalent to $\mathrm{AMT}$ over $\mathrm{RCA}_{0}$; while if $\mathcal{C}$ denotes the pairwise full, pairwise free, or 1-point full amalgamation closure conditions, then $\mathrm{EFAE}_{\mathcal{C}}$ is somewhere between AMT and ATT in strength.

Let $\mathcal{P}$ be one of SMT, WMT, 1-PHMT, 1-HMT, or HMT, and let $\mathcal{C}_{\mathcal{P}}$ be the corresponding set of closure conditions. By Proposition 6.6, $\mathcal{P}$ implies $\mathrm{EFAE}_{\mathcal{C}_{\mathcal{P}}}$ over $\mathrm{RCA}_{0}$.

By the first two parts of Theorem 6.10, it is also provable in $\mathrm{RCA}_{0}$ that if a list of types $X$ satisfies the finite free (finite full) amalgamation closure conditions and has an extension function approximation, then there is a (homogeneous) model $\mathcal{A}$ such that $X$ is an enumeration of the type spectrum of $\mathcal{A}$. Thus WMT and HMT are each equivalent to $\mathrm{EFAE}_{\mathcal{C}_{\mathcal{P}}}$ for the corresponding set of closure conditions, and hence to AMT.

By the last two parts of Theorem 6.10, it is provable in $\mathrm{RCA}_{0}+\Pi_{1}^{0} \mathrm{GA}$ that if a list of types $X$ satisfies the pairwise free (pairwise full) amalgamation closure conditions and has an extension function approximation, then there is a (1-homogeneous) model $\mathcal{A}$ such that $X$ is an enumeration of the type spectrum of $\mathcal{A}$. Thus SMT and 1-HMT follow from $\mathrm{EFAE}_{\mathcal{C}_{\mathcal{P}}}$ for the corresponding set of closure conditions over $\mathrm{RCA}_{0}+\Pi_{1}^{0} \mathrm{GA}$, and hence are somewhere between AMT and ATT $+\Pi_{1}^{0} \mathrm{GA}$ in strength.

We also show, in Theorem 7.7, that 1-PHMT is equivalent to $\mathrm{AMT}+\mathrm{I} \Sigma_{2}^{0}$. 


\subsection{Extension function approximations, AMT, and ATT}

The following theorems compare our $\mathrm{EFAE}_{\mathcal{C}}$ principles with AMT and ATT.

THEOREM 7.1. (1) Let $\mathcal{C}$ denote the finite free amalgamation closure conditions. Then AMT implies $\mathrm{EFAE}_{\mathcal{C}}$ over $\mathrm{RCA}_{0}$.

(2) Let $\mathcal{C}$ be any set of closure conditions containing the extension condition. Then ATT implies $\mathrm{EFAE}_{\mathcal{C}}$ over $\mathrm{RCA}_{0}$.

ProOF. The proofs of the two parts of the theorem are almost identical. We first prove part 1 , then indicate the changes necessary to prove part 2. As mentioned in Chapter 3, we can take AMT as stating that for every strongly atomic extendible tree $\mathcal{T}$, there is a listing of the paths of $\mathcal{T}$. Let $T$ be a theory and $X=\left\{p_{i}\right\}_{i \in \mathbb{N}}$ a list of elements of $S(T)$ satisfying the finite free amalgamation closure conditions. Arguing in $\mathrm{RCA}_{0}$, we define a family of trees $\mathcal{T}_{i, j}$, and put them together to form a strongly atomic extendible tree $\mathcal{T}$ so that any enumeration of the isolated paths of $\mathcal{T}$ can be used to define an extension function approximation for $X$.

Given an $n$-type $p_{i}$, an $(n+l)$-ary formula $\psi$ consistent with $p_{i}$, and an $s$, we say that the $(n+l)$-type $p_{j}$ amalgamates $p_{i}$ and $\psi$ at stage $s$ if the conjunction of $\psi$ and the formulas in $p_{j}\left\lceil s\right.$ is consistent with $p_{i}$. Such a $p_{j}$ always exists, since $X$ satisfies the extension condition.

Let $\theta_{0}, \theta_{1}, \ldots$ be the formulas in the language of $T$. If $p_{i}$ and $\theta_{j}$ are inconsistent, or not of the form $p_{i}(\bar{x})$ and $\theta_{j}(\bar{x} \bar{y})$, then let $\mathcal{T}_{i, j}=\left\{1^{k}: k \in \mathbb{N}\right\}$. Otherwise, let $\mathcal{T}_{i, j}=\bigcup_{s \in \mathbb{N}} \mathcal{T}_{i, j}^{s}$, where $\mathcal{T}_{i, j}^{s}$ is defined in stages as follows.

Stage 0. Let $\psi_{0}=\theta_{j}$. Let $\mathcal{T}_{i, j}^{0}=\left\{1^{k}: k \in \mathbb{N}\right\}$. We associate with each node $\tau=1^{k}$ in $\mathcal{T}_{i, j}^{0}$ a pair $s_{\tau}=k$ and $t_{\tau}=0$, and a formula $\psi_{\tau}=\theta_{j}$. We label all the nodes $\tau$ in $\mathcal{T}_{i, j}^{0}$ as active.

Stage $s+1$. We are given the tree $\mathcal{T}_{i, j}^{s}$, and for each $\tau \in \mathcal{T}_{i, j}^{s}$, we have defined

(1) $s_{\tau} \quad$ (the guide amalgamator being followed),

(2) $t_{\tau} \quad$ (the length of time $s_{\tau}$ has been followed), and

(3) $\psi_{\tau}$ consistent with $p_{i} \cup\left\{\theta_{j}\right\} \quad$ (the guide formula),

where $p_{s_{\tau}}$ amalgamates $p_{i}$ and $\psi_{\tau}$ at stage $t_{\tau}$.

We obtain $\mathcal{T}_{i, j}^{s+1}$ by adding nodes to $\mathcal{T}_{i, j}^{s}$. For every active $\tau \in \mathcal{T}_{i, j}^{s}$, check whether $p_{s_{\tau}}$ amalgamates $p_{i}$ and $\psi_{\tau}$ at stage $t_{\tau}+1$. If so, add $\tau 0$ to $\mathcal{T}_{i, j}^{s+1}$, let $s_{\tau 0}=s_{\tau}$, let $t_{\tau 0}=t_{\tau}+1$, let $\psi_{\tau 0}=\psi_{\tau}$, deactivate $\tau$, and activate $\tau 0$.

If $p_{s_{\tau}}$ does not amalgamate $p_{i}$ and $\psi_{\tau}$ at stage $t_{\tau}+1$, add $\tau 1^{k+1}$ to $\mathcal{T}_{i, j}^{s+1}$ for all $k \in \mathbb{N}$. For each $\tau^{\prime}=\tau 1^{k+1}$, let $s_{\tau^{\prime}}=k$ and $t_{\tau^{\prime}}=0$, and let $\psi_{\tau^{\prime}}$ be the conjunction of the formulas in $p_{s_{\tau}} \uparrow t_{\tau}$ and $\psi_{\tau}$. Deactivate $\tau$, and activate each such $\tau^{\prime}$.

Let $\mathcal{T}=\left\{0^{n}: n \in \mathbb{N}\right\} \cup\left\{0^{\langle i, j\rangle} 1 \sigma: \sigma \in \mathcal{T}_{i, j}\right\}$. It is easy to see that the existence of $\mathcal{T}$ can be established in $\mathrm{RCA}_{0}$.

LEMma 7.2. The tree $\mathcal{T}$ is extendible and strongly atomic.

ProOF. Extendibility is clear from the definition. Now let $\rho_{0}, \ldots, \rho_{n} \in \mathcal{T}$. By extending $\rho_{k}$ if necessary, we may assume that each $\rho_{k}$ is of the form $0^{\left\langle i_{k}, j_{k}\right\rangle} 1 \tau_{k}$ for some $\tau_{k} \in \mathcal{T}_{i_{k}, j_{k}}$.

Let $P$ be the property that holds of $k \leqslant n$ if and only if $p_{i_{k}}$ and $\theta_{j_{k}}$ are inconsistent or not of the form $p_{i}(\bar{x})$ and $\theta_{j}(\bar{x} \bar{y})$, and let $Q$ be the property that 
holds of $k \leqslant n$ if and only if $p_{s_{\tau_{k}}}$ amalgamates $p_{i_{k}}$ and $\psi_{\tau_{k}}$. These properties are $\Delta_{0}^{0}$ and $\Pi_{1}^{0}$, respectively, so by bounded $\Pi_{1}^{0}$-comprehension, we can split our sequence of $\rho$ 's into three subsequences, one consisting of the $\rho_{k}$ 's such that $P(k)$ holds, another of the $\rho_{k}$ 's such that $Q(k)$ holds, and the last of all other $\rho_{k}$ 's. It is enough to find atoms extending the $\rho$ 's in each of these sequences independently.

If $P(k)$ holds then $\rho_{k}$ is itself an atom of $\mathcal{T}$. If $Q(k)$ holds then it follows easily by induction that the extensions of $\tau_{k}$ in $\mathcal{T}_{i_{k}, j_{k}}$ are exactly those of the form $\tau_{k} 0^{l}$, so again $\rho_{k}$ is an atom of $\mathcal{T}$.

Thus we may assume that we have a sequence $\rho_{0}, \ldots, \rho_{n}$ as above such that for each $k \leqslant n$, the type $p_{s_{\tau_{k}}}$ does not amalgamate $p_{i_{k}}$ and $\psi_{\tau_{k}}$. Let $r_{k}$ be the stage at which $\tau_{k}$ is declared active and let $t_{k} \geqslant r_{k}$ be least such that $p_{s_{\tau_{k}}}$ does not amalgamate $p_{i_{k}}$ and $\psi_{\tau_{k}}$ at stage $t_{k}+1$. Then $\nu_{k}=\tau_{k} 0^{t_{k}-r_{k}} \in \mathcal{T}_{i_{k}, j_{k}}$, and we have $s_{\nu_{k}}=s_{\tau_{k}}, t_{\nu_{k}}=t_{k}$, and $\psi_{\nu_{k}}=\psi_{\tau_{k}}$. Furthermore, for each $l$, we have $\sigma_{k, l}=\nu_{k} 1^{l+1} \in \mathcal{T}_{i_{k}, j_{k}}$. All the formulas $\psi_{\sigma_{k, l}}$ are the same formula $\varphi_{k}$, which by induction is consistent with $p_{i_{k}}$. As noted in Remark 5.9, there are $m_{0}, \ldots, m_{n}$ such that $p_{m_{k}}$ amalgamates $p_{i_{k}}$ and $\varphi_{k}$. Then $0^{\left\langle i_{k}, j_{k}\right\rangle} 1 \sigma_{k, m_{k}}$ is an atom of $\mathcal{T}$ extending $\rho_{k}$ for each $k \leqslant n$.

LEMMA 7.3. An extension function approximation for $X$ can be constructed from any enumeration of the isolated paths of $\mathcal{T}$.

Proof. Let $f_{0}, f_{1}, \ldots$ be an enumeration of the isolated paths of $\mathcal{T}$. Let $i$ and $j$ be such that $\theta_{j}$ is consistent with $p_{i}$. Since $\mathcal{T}$ is atomic, there is a least $n$ such that $f_{n}$ extends $0^{\langle i, j\rangle} 1$. Let $f(k)=f_{n}(k+\langle i, j\rangle+1)$. Then $f$ is an isolated path of $\mathcal{T}_{i, j}$. By the construction of $\mathcal{T}_{i, j}$, any $\tau \in \mathcal{T}$ such that $\tau(|\tau|-1)=1$ has more than one extension in $\mathcal{T}$, so $f=\tau 0^{\mathbb{N}}$ for some $\tau \in 2^{<\mathbb{N}}$.

Let $m$ be such that $f$ extends $1^{m} 0$. Let $\tau_{0}=1^{m} 0$ and let $g(i, j, 0)=m$. Given $g(i, j, s)$ and $\tau_{s}$, proceed as follows. If $f$ extends $\tau_{s} 0$ then let $\tau_{s+1}=\tau_{s} 0$ and $g(i, j, s+1)=s_{\tau_{s}}=s_{\tau_{s+1}}$. Otherwise, let $\tau_{s+1}$ be the shortest string extended by $f$ of the form $\nu 01^{k} 0^{s+1}$, where $k \geqslant 1$ and $|\nu| \geqslant\left|\tau_{s}\right|-2$. It is easy to see that such a string must exist, and that $s_{\tau_{s+1}}=k$. Let $g(i, j, s+1)=k=s_{\tau_{s+1}}$.

There are only finitely many $s$ such that $f$ extends $\tau_{s} 1$, and hence $\lim _{s} g(i, j, s)$ exists. It follows from the construction of $\mathcal{T}_{i, j}$ that $g$ is an extension function approximation.

This concludes the proof of part 1 of the theorem. The only place in this proof where any property of $\mathcal{C}$ other than containing the extension condition is used is at the end of the proof of Lemma 7.2, to justify the existence of $m_{0}, \ldots, m_{n}$. Thus, if we restrict ourselves to the $n=0$ case of this lemma, all we need is for $\mathcal{C}$ to contain the extension condition. This case is enough to conclude that $\mathcal{T}$ is atomic, which gives us part 2 of the theorem.

THEOREM 7.4. Let $\mathcal{C}$ denote the finite full amalgamation closure conditions. Then $\mathrm{EFAE}_{\mathcal{C}}$ implies AMT over $\mathrm{RCA}_{0}$.

Proof. We argue in $\mathrm{RCA}_{0}$. Let $\mathcal{T}$ be a strongly atomic extendible tree. We define a set of axioms $A$ whose deductive closure $\Delta$ is a theory. Then we define a list $X$ of types of $\Delta$ satisfying the finite full amalgamation closure conditions, so that from an extension function approximation for $X$, we can construct a listing of the isolated paths of $\mathcal{T}$. 
Axioms. We work in the language with unary relation symbols $U, R_{i}$ for $i \in \mathbb{N}$, and $D_{\sigma}$ for $\sigma \in \mathcal{T}$; and binary relation symbols $P$ and $E$. We first define the following set $A$ of axioms and then discuss the intuition behind them.

A1. $P(x, y) \rightarrow \neg U(x) \wedge U(y)$.

A2. $\neg U(x) \rightarrow\left(\exists^{=1} y\right) P(x, y)$.

A3. $(\forall y)\left[U(y) \rightarrow\left(\exists^{\geqslant k} x\right) P(x, y)\right]$ for all $k \in \mathbb{N}$.

A4. $E\left(x_{0}, x_{1}\right) \leftrightarrow(\exists y)\left[P\left(x_{0}, y\right) \wedge P\left(x_{1}, y\right)\right]$.

A5. $D_{\sigma}(x) \rightarrow \neg U(x)$ for all $\sigma \in \mathcal{T}$.

A6. $D_{\sigma}(x) \rightarrow D_{\tau}(x)$ for all $\tau \prec \sigma \in \mathcal{T}$.

A7. $D_{\sigma}(x) \rightarrow \neg D_{\tau}(x)$ for all $\sigma, \tau \in \mathcal{T}$ such that $|\sigma|=|\tau|$ and $\sigma \neq \tau$.

A8. $R_{i}(y) \rightarrow U(y)$ for all $i \in \mathbb{N}$.

A9. $R_{i}(y) \rightarrow R_{i+1}(y)$ for all $i \in \mathbb{N}$.

A10. $\left(\exists^{\geqslant k} y\right) R_{0}(y)$ for all $k \in \mathbb{N}$.

A11. $\left(\exists^{\geqslant k} y\right)\left[R_{i+1}(y) \wedge \neg R_{i}(y)\right]$ for all $i, k \in \mathbb{N}$.

A12. $\neg R_{n}(y) \rightarrow\left(\exists^{\geqslant k} x\right)\left[D_{\sigma}(x) \wedge P(x, y)\right]$ for all $n, k \in \mathbb{N}$ and all $\sigma \in \mathcal{T}$ such that $|\sigma|=n$.

A13. $\left[\neg U(x) \wedge \bigwedge_{\sigma \in S_{n}} \neg D_{\sigma}(x)\right] \leftrightarrow(\exists y)\left[R_{n}(y) \wedge P(x, y)\right]$ for all $n \in \mathbb{N}$, where $S_{n}$ is the set of elements of $\mathcal{T}$ of length $n$.

Axiom groups A1, A2, and A3 tell us that we can divide our universe into two disjoint parts, $\mathcal{D}=\{x: \neg U(x)\}$ and $\mathcal{R}=\{x: U(x)\}$, and that $P$ determines a function with domain $\mathcal{D}$ and range $\mathcal{R}$ such that the preimage of any $y \in \mathcal{R}$ is infinite. Axiom A4 tells us that $E$ is the equivalence relation on $\mathcal{D}$ wherein two elements are equivalent if they have the same image under $P$. (Having a symbol for this relation is needed in establishing effective quantifier elimination.)

Axiom groups A5, A6, and A7 tell us that the $D_{\sigma}$ hold only of elements of $\mathcal{D}$, and that for each $x \in \mathcal{D}$, the set of $\sigma$ such that $D_{\sigma}(x)$ holds is either empty, of the form $\{\sigma: \sigma \preccurlyeq \tau\}$ for some $\tau \in \mathcal{T}$, or of the form $\{\sigma: \sigma \prec f\}$ for some $f \in[\mathcal{T}]$.

Axiom groups A8, A9, A10, and A11 tell us that the $R_{i}$ hold only of elements of $\mathcal{R}$; that for each $y \in \mathcal{R}$, the set of $i$ such that $R_{i}(x)$ holds is either empty or consists of all $i \geqslant j$ for some $j$; and that for each $j$ there are infinitely many $y$ such that this set is of the latter form.

Finally, axiom groups A12 and A13 connect the set of $\sigma$ such that $D_{\sigma}(x)$ holds with the set of $i$ such that $R_{i}(y)$ holds, for $x$ and $y$ such that $P(x, y)$ holds. They say that if $R_{n}(y)$ holds then there is no $\sigma \in \mathcal{T}$ of length $n$ and no $x$ in the $P$ preimage of $y$ such that $D_{\sigma}(x)$ holds, while if $R_{n}(y)$ fails, then for every $x$ in the $P$-preimage of $y$, some $D_{\sigma}(x)$ with $\sigma$ of length $n$ holds (because in this case, by $\mathrm{A} 2$, the right hand side of A13 fails, and hence so does the left hand side), and for 
every $\sigma \in \mathcal{T}$ of length $n$ there are infinitely many $x$ in the $P$-preimage of $y$ such that $D_{\sigma}(x)$ holds.

We show that $A$ is consistent and admits effective quantifier elimination, so that its deductive closure $\Delta$ exists and is a theory. We then give a list of types $X$ of $\Delta$ satisfying the finite full amalgamation closure conditions, and use the existence of an extension function approximation for $X$ to obtain a listing of the isolated paths of $\mathcal{T}$. The idea is to ensure the following two properties. Every 1-type in $X$ containing $\neg U(x)$ contains $D_{\sigma}(x)$ either for only finitely many $\sigma$ or for all $\sigma \prec f$ for an isolated path $f$ of $\mathcal{T}$; and there is a 1-type $p(y)$ in $X$ containing $U(y)$ and $\neg R_{n}(y)$ for all $n$. Then for any $\tau \in \mathcal{T}$, we can use the given extension function approximation for $X$ to obtain an amalgamator $q(x, y) \in X$ for $p(y)$ and the formula $D_{\tau}(x) \wedge P(x, y)$. By the axioms and the properties of $X$, the set of $\sigma$ such that $D_{\sigma}(x) \in q(x, y)$ must determine an isolated path of $\mathcal{T}$ extending $\tau$.

Quantifier elimination. We begin by showing that $A$ admits effective quantifier elimination, as discussed at the end of Section 2.2. Fix an existential formula $(\exists y) \varphi(\bar{x} y)$, where $\varphi(\bar{x} y)$ is a conjunction of literals. Let $m$ be such that no $R_{i}$ with $i>m$ or $D_{\sigma}$ with $|\sigma|>m$ occurs in $\varphi$, and let $\mathcal{L}^{\prime}$ be the language consisting of $U$, $P, E$, all $R_{i}$ with $i \leqslant m$, and all $D_{\sigma}$ with $\sigma \in \mathcal{T}$ and $|\sigma| \leqslant m$. As mentioned in Remark 2.3, we may assume that $\varphi$ is an $\mathcal{L}^{\prime}$-atomic diagram that includes $\neg u=v$ for all distinct $u, v \in \bar{x} y$. By a similar argument to the one mentioned at the end of that section, we may also assume that $\varphi$ is consistent with the assertion that $E$ is an equivalence relation on the set of $u$ such that $\neg U(u)$ holds.

Suppose that at least one of the following conditions holds.

(1) $P(u, v)$ occurs in $\varphi$, and either $U(u)$ or $\neg U(v)$ occurs in $\varphi$.

(2) $P(u, v)$ and $P(u, w)$ both occur in $\varphi$, where $v$ and $w$ are distinct.

(3) $P(u, w), P(v, w)$, and $\neg E(u, v)$ all occur in $\varphi$.

(4) $P(u, w), \neg P(v, w)$, and $E(u, v)$ all occur in $\varphi$.

(5) $E(u, v)$ and $U(u)$ both occur in $\varphi$.

(6) $\neg E(u, u)$ and $\neg U(u)$ both occur in $\varphi$.

(7) $D_{\sigma}(u)$ and $U(u)$ both occur in $\varphi$.

(8) $D_{\sigma}(u)$ and $\neg D_{\tau}(u)$ for $\tau \prec \sigma$ both occur in $\varphi$.

(9) $D_{\sigma}(u)$ and $D_{\tau}(u)$, where $|\sigma|=|\tau|$ and $\sigma \neq \tau$, both occur in $\varphi$.

(10) $R_{i}(u)$ and $\neg U(u)$ both occur in $\varphi$.

(11) $R_{i}(u)$ and $\neg R_{j}(u)$ with $i<j$ both occur in $\varphi$.

(12) $D_{\sigma}(u), P(u, v)$, and $R_{|\sigma|}(v)$ all occur in $\varphi$.

(13) $E(u, v), D_{\sigma}(u)$, and $\neg D_{\tau}(v)$ for all $\tau$ such that $|\tau|=|\sigma|$, all occur in $\varphi$.

Then $A \vdash(\exists y) \varphi(\bar{x} y) \leftrightarrow$ F. (For (1)-(12), this fact is easy to see from the axioms. For $(13)$, assume that $E(u, v)$ and $D_{\sigma}(u)$ occurs in $\varphi$. We may assume that $\neg U(u)$ and $\neg U(v)$ both occur in $\varphi$, as otherwise $\varphi$ satisfies (5). Then $T \cup\{\varphi\}$ implies the negation of the left hand side of A13 for $x=u$ and $n=|\sigma|$, and hence the negation of the right hand side of A13 for $x=u$ and $n=|\sigma|$. By A4, $T \cup\{\varphi\}$ implies the negation of the right hand side of A13 for $x=v$ and $n=|\sigma|$, hence the negation of the left hand side of A13 for $x=v$ and $n=|\sigma|$, and hence $D_{\tau}(v)$ for some $\tau$ such that $|\tau|=|\sigma|$.)

Otherwise, let $\mathcal{D}$ be the set of variables $u$ such that $\neg U(u)$ occurs in $\varphi$ and let $\mathcal{R}$ be the set of variables $v$ such that $U(v)$ occurs in $\varphi$. For each $v \in \mathcal{R}$, let $\mathcal{D}_{v}$ be the set of variables $u$ such that $P(u, v)$ occurs in $\varphi$. Note that the sets $\mathcal{D}_{v}$ are 
pairwise disjoint. Let $\mathcal{D}_{\emptyset}$ be the set of all elements of $\mathcal{D}$ that are not in any $\mathcal{D}_{v}$. Let $\theta(\bar{x})$ be the result of removing all literals mentioning $y$ from $\varphi$.

If $y \in \mathcal{R}$ then the only positive literals in $\varphi$ involving $y$ and another variable are the ones of the form $P(x, y)$ for $x \in \mathcal{D}_{y}$. If $x, x^{\prime} \in \mathcal{D}_{y}$, then $E\left(x, x^{\prime}\right)$ occurs in $\varphi$, and if $x \in \mathcal{D}_{y}$, then $R_{i}(y)$ occurs in $\varphi$ if and only if $\neg D_{\sigma}(x)$ occurs in $\varphi$ for all $\sigma \in \mathcal{T}$ of length $i$. Combining A2, A4, and A13, we see that $A \vdash(\exists y) \varphi(\bar{x} y) \leftrightarrow \theta(\bar{x})$.

If $y \in \mathcal{D}_{v}$, where $v$ is either a variable or $\emptyset$, then the only positive literals in $\varphi$ involving $y$ and another variable are ones of the form $E(x, y)$ or $E(y, x)$ with $x \in \mathcal{D}_{v}$, and $P(y, v)$ if $v$ is a variable. Let $x \in \mathcal{D}_{v}$ and $i \leqslant m$. Then $D_{\sigma}(x)$ occurs in $\varphi$ for some $\sigma$ of length $i$ if and only if $D_{\tau}(y)$ occurs in $\varphi$ for some $\tau$ of length $i$. Thus, by A2, A3, A4, A12, and A13, we again have $A \vdash(\exists y) \varphi(\bar{x} y) \leftrightarrow \theta(\bar{x})$.

We have completed the quantifier elimination process, and hence conclude that the deductive closure $\Delta$ of $A$ exists and is a theory.

Consistency. We now establish the consistency of $\Delta$ by providing a model $\mathcal{M}$. By effective quantifier elimination, to define $\mathcal{M}$, it suffices to specify the interpretations of the symbols of our language in $\mathcal{M}$. Let $\sigma_{0}, \sigma_{1}, \ldots$ be a listing of the elements of $\mathcal{T}$.

(1) Let $M=\mathbb{N}$.

(2) Let $U^{\mathcal{M}}$ be the odd numbers.

(3) Let $D_{\tau}^{\mathcal{M}}=\left\{2\langle i, j, m\rangle: \tau \preccurlyeq \sigma_{i}\right\}$.

(4) Let $R_{n}^{\mathcal{M}}=\{2\langle k, j\rangle+1: n \geqslant k\}$.

(5) Let $P^{\mathcal{M}}=\left\{(2\langle i, j, m\rangle, 2\langle k, j\rangle+1):\left|\sigma_{i}\right|=k-1\right\}$.

(6) Let $E^{\mathcal{M}}=\{(2\langle i, j, m\rangle, 2\langle i, j, n\rangle): m, n \in \mathbb{N}\}$.

It is easy to check that $\mathrm{A} 1-\mathrm{A} 13$ hold in this model. (Indeed, $\mathcal{M}$ is an atomic model of the theory induced by these axioms, though this fact is not needed in our proof.)

Defining the list of types $X$. We now proceed to define a list $X$ of types of $\Delta$. By effective quantifier elimination, we can identify types of $\Delta$ with atomic types. Notice that the 0 -type $\Delta$ corresponds to the empty atomic 0 -type. From now on, we refer to an atomic type as simply a type.

We define a few particular 1-types in a given variable $x$. Each 1-type in $x$ includes $x=x$ and $\neg P(x, x)$. If it includes $\neg U(x)$, then it includes $E(x, x)$ and $\neg R_{i}(x)$ for all $i \in \mathbb{N}$. If it includes $U(x)$, then it includes $\neg E(x, x)$ and $\neg D_{\sigma}(x)$ for all $\sigma \in \mathcal{T}$.

Let $i \in \mathbb{N}$. Let $r_{i}(x)$ be the principal type generated by $\neg R_{i-1}(x) \wedge R_{i}(x)$, or just $R_{0}(x)$ if $i=0$. Let $s(x)$ be the nonprincipal type that includes $U(x)$ and $\neg R_{j}(x)$ for all $j \in \mathbb{N}$.

Let $\sigma \in \mathcal{T}$. If $\sigma$ is not the empty string $\lambda$, let $\sigma^{-}$be $\sigma \uparrow|\sigma|-1$. Let $d_{\sigma}(x)$ be the principal type generated by $D_{\sigma^{-}}(x) \wedge \neg D_{\sigma}(x)$. Let $d_{\lambda}(x)$ be the principal type generated by $\neg U(x) \wedge \neg D_{\lambda}(x)$.

Let $e_{\sigma}(x)$ be the type defined as follows. First, $e_{\sigma}(x)$ includes $\neg U(x), D_{\tau}(x)$ for every $\tau \preccurlyeq \sigma$, and $\neg D_{\tau}(x)$ for every $\tau \in \mathcal{T}$ incompatible with $\sigma$. Next, for each $\tau \in \mathcal{T}$ extending $\sigma$, if $\tau$ is the only extension of $\sigma$ of length $|\tau|$ in $\mathcal{T}$, then $e_{\sigma}(x)$ includes $D_{\tau}(x)$. Otherwise, $e_{\sigma}(x)$ includes $\neg D_{\rho}(x)$ for every extension $\rho$ of $\sigma$ of length $|\tau|$ in $\mathcal{T}$, including $\tau$ itself. Notice that if $\sigma$ is not an atom, then $e_{\sigma}(x)$ is equal to $d_{\tau}(x)$ for some $\tau$. Otherwise, the set of $\tau$ such that $D_{\tau}(x) \in e_{\sigma}(x)$ determines an isolated path of $\mathcal{T}$.

Let $B$ be the set of 1-types described above. These types (where $x$ ranges over all variables) are exactly the 1 -types in $X$. Notice that they include every principal 
1-type of $\Delta$, and every 1-type of $\Delta$ extending $U(x)$. To describe the $n$-types in $X$ for $n>1$, we first define a few operators. It is straightforward to check from the axioms that operators with the stated properties exist.

Our first operator gives us a way to extend a formula to a type in a safe way, i.e., without introducing any unwanted 1-types. For a conjunction of literals $\theta$ consistent with $\Delta$, let $\mathcal{J}(\theta)$ be a type containing the literals in $\theta$ such that for each variable $x$, there are at most finitely many $\sigma$ for which $D_{\sigma}(x) \in \mathcal{J}(\theta)$. Notice that the 1-subtypes of $\mathcal{J}(\theta)$ must be of the form $d_{\sigma}, r_{i}$, or $s$.

Our second operator gives us a way to transform a possibly inconsistent complete set of literals into a type. Let $q(\bar{x})$ be a set of literals that, for every atomic formula $\varphi$ with free variables in $\bar{x}$, includes either $\varphi$ or $\neg \varphi$ (and possibly both). Let $\varphi_{0}, \varphi_{1}, \ldots$ be the elements of $q$ (ordered according to a standard fixed ordering of literals). If there is an $n$ such that $\left\{\varphi_{0}, \ldots, \varphi_{n}\right\}$ is not consistent with $\Delta$, then for the least such $n$, let $\mathcal{P}(q)=\mathcal{J}\left(\bigwedge_{i<n} \varphi_{i}\right)$. Otherwise, let $\mathcal{P}(q)=q$. Note that this operator is effective, since to know whether a given literal $\varphi$ is in $\mathcal{P}(q)$, we need only find an $m$ such that $\varphi_{m}$ is either $\varphi$ or its negation. If $\left\{\varphi_{0}, \ldots, \varphi_{m}\right\}$ is consistent with $\Delta$ then $\varphi \in \mathcal{P}(q)$ if and only in $\varphi_{m} \equiv \varphi$. Otherwise we check whether $\varphi$ is in $\mathcal{J}\left(\bigwedge_{i<n} \varphi_{i}\right)$ for the least $n$ such that $\left\{\varphi_{0}, \ldots, \varphi_{n}\right\}$ is not consistent with $\Delta$, which must exist.

Our third operator performs amalgamation. Let $q_{0}\left(\bar{x}_{0}\right), \ldots, q_{n}\left(\bar{x}_{n}\right)$ be types, let $\bar{x}$ be a tuple such that $\bar{x}=\bar{x}_{0} \cup \cdots \cup \bar{x}_{n}$, and let $q=q_{0} \cup \cdots \cup q_{n}$. Let $\theta(\bar{x})$ be a conjunction of literals such that for each pair of distinct variables $u, v \in \bar{x}$, either $P(u, v)$ or $\neg P(u, v)$ is in $\theta$, either $E(u, v)$ or $\neg E(u, v)$ is in $\theta$, and either $u=v$ or $\neg u=v$ is in $\theta$. Let $r$ be the union of $q$ with the literals in $\theta$. Note that if $r$ is consistent with $\Delta$, then it is in fact a type, and amalgamates $q_{0}, \ldots, q_{n}$. Let $\mathcal{A}\left(q_{0}, \ldots, q_{n}, \theta\right)=\mathcal{P}(r)$.

Our fourth operator extracts subtypes and performs variable substitutions. Let $V$ be the set of variables in our language. By a finite injective partial function on variables we mean a finite subset $f$ of $V^{2}$ that satisfies the properties of an injective function. We use the usual functional notation when discussing $f$, and in particular write $f(x) \downarrow$ to mean that $x$ is in the domain of $f$. Let $q\left(x_{0}, \ldots, x_{n}\right)$ be a type and let $f$ be an finite injective partial function on variables. Let $S$ be the set of $x_{i}$ with $i \leqslant n$ such that $f\left(x_{i}\right) \downarrow$. If $x_{i} \in S$ then let $g\left(x_{i}\right)=f\left(x_{i}\right)$. Define $g$ on all other $x_{i}$ with $i \leqslant n$ so that $g$ is a finite injective partial function on variables. Let $\mathcal{S}(q, f)=q\left[g\left(x_{0}\right), \ldots, g\left(x_{n}\right)\right]\lceil S$ (using the notation introduced in Remark 5.9).

We define $X$ recursively. Even if we can ensure that we do not introduce any unwanted 1-types at each step, we do not have sufficient induction to conclude that no unwanted 1-types ever appear. Our final operator allows us to ensure that this is indeed the case by transforming an arbitrary type into one whose 1-subtypes are all of the desired form. Let $q\left(x_{0}, \ldots, x_{k-1}\right)$ be a type and $\sigma_{0}, \ldots, \sigma_{k-1} \in \mathcal{T}$. Let $\varphi_{0}, \varphi_{1}, \ldots$ be the elements of $q$ (ordered according to a standard fixed ordering of literals). If there are an $n$ and a $j<k$ such that $\varphi_{n} \equiv D_{\tau}\left(x_{j}\right)$ and either $\tau$ is incompatible with $\sigma_{j}$ or there is more than one extension of $\sigma_{j}$ of length $\tau$ in $\mathcal{T}$, then for the least such $n$, let $\mathcal{N}\left(q, \sigma_{0}, \ldots, \sigma_{k-1}\right)=\mathcal{J}\left(\bigwedge_{i<n} \varphi_{i}\right)$. Otherwise, let $\mathcal{N}\left(q, \sigma_{0}, \ldots, \sigma_{k-1}\right)=q$. Notice that, in either case, the 1-subtypes of $\mathcal{N}\left(q, \sigma_{0}, \ldots, \sigma_{k-1}\right)$ are all in $B$.

We are now ready to define $X=\left\{p_{i}\right\}_{i \in \mathbb{N}}$. Let $p_{\langle 0, k\rangle}=\emptyset$ for all $k \in \mathbb{N}$. Let $t_{0}, t_{1}, \ldots$ be a listing of $B$, let $\sigma_{0}, \sigma_{1}, \ldots$ be a listing of $\mathcal{T}$, let $x_{0}, x_{1}, \ldots$ be 
the variables in our language, and let $p_{\langle 1, k\rangle}=\mathcal{N}\left(t_{i}\left(x_{j}\right), \sigma_{m}\right)$ where $k=\langle i, j, m\rangle$. (While we do not need to apply the $\mathcal{N}$ operator here, it is helpful for uniformity of definition. Notice that for each $t_{i}$, there is a $\sigma$ such that $\mathcal{N}\left(t_{i}, \sigma\right)=t_{i}$.)

Having defined the types $p_{\langle m, k\rangle}$ for $m<n$, we define the types of the form $p_{\langle n, k\rangle}$ to consist of all possible amalgamations of previously defined types, together with all types obtained from these amalgamations by permuting variables or taking subtypes. We use the $\mathcal{N}$ operator to ensure that we do not create any unwanted 1-types.

Let $\mathcal{F}$ be the set of 4 -tuples consisting of a finite set $G$ of numbers of the form $\langle m, k\rangle$ with $m<n$, a conjunction of literals in the variables $\bar{x}$ appearing in the union of the types $p_{c}$ for $c \in G$, a sequence $\sigma_{0}, \ldots, \sigma_{|\bar{x}|-1}$ of elements of $\mathcal{T}$, and a finite injective partial function on variables. Fix a listing of $\mathcal{F}$, and let $G_{0}, G_{1}, \ldots$ be the corresponding finite sets, $\theta_{0}, \theta_{1}, \ldots$ the corresponding formulas, $s_{0}, s_{1}, \ldots$ the corresponding sequences of strings, and $f_{0}, f_{1}, \ldots$ the corresponding partial functions. For each $i$, let $q_{0}, \ldots, q_{l}$ be the types $p_{c}$ such that $c \in G_{i}$, and let $p_{\langle n, i\rangle}=\mathcal{S}\left(\mathcal{N}\left(\mathcal{A}\left(q_{0}, \ldots, q_{l}, \theta_{i}\right), s_{i}\right), f_{i}\right)$.

Let $X=\left\{p_{i}\right\}_{i \in \mathbb{N}}$. Then $X$ is a list of types of $\Delta$, and, because of our application of the $\mathcal{N}$ operator, every 1 -type in $X$ is in $B$. We claim that $X$ satisfies the finite full amalgamation closure conditions.

First, $\emptyset \in X$. Closure under sequences of variable substitutions, and hence closure under variable substitutions and under subtypes, follows from the fact that each time a type is added to $X$, so are all the types obtained from it by applications of the $\mathcal{S}$ operator, in an effective way.

It is also easy to see that for a finite set of elements $q_{0}, \ldots, q_{n}$ of $X$, if there is a type of $\Delta$ containing $q_{0} \cup \cdots \cup q_{n}$, then there is a conjunction of literals $\theta$ such that $\mathcal{A}\left(q_{0}, \ldots, q_{n}, \theta\right)$ amalgamates these types. Furthermore, since the 1 -subtypes of $q_{0} \cup \cdots \cup q_{n}$ are all in $B$, the fact that $\mathcal{T}$ is strongly atomic means that there is a sequence $t$ of elements of $\mathcal{T}$ such that $\mathcal{N}\left(\mathcal{A}\left(q_{0}, \ldots, q_{n}, \theta\right), t\right)=\mathcal{A}\left(q_{0}, \ldots, q_{n}, \theta\right)$. Thus finite full type amalgamation holds.

To verify that the extension condition is satisfied, it is enough to fix a $p(\bar{x}) \in X$ and a conjunction of literals $\theta(\bar{x} \bar{y})$ consistent with $p$, and show that there is a $q(\bar{x} \bar{y}) \in X$ containing both. We do so by defining a type $t(u)$ for each $u \in \bar{y}$, and then applying the $\mathcal{A}$ operator to $p$, the types $t(u)$, and $\theta$.

By extending $\theta$ if needed, we may assume that $p \cup\{\theta\}$ decides every atomic formula in its free variables involving $P, E$, or $U$ (i.e., it includes either the formula or its negation). We may also assume that if it includes $R_{i}(u)$ then it decides $R_{j}(u)$ for all $j \leqslant i$, and that if it includes $\neg D_{\sigma}(u)$, then it decides $D_{\tau}(u)$ for all $\tau \preccurlyeq \sigma$. We may also assume that $u=v$ does not occur in $p \cup\{\theta\}$ for any distinct variables $u$ and $v$, and hence that $\neg u=v$ occurs in $p \cup\{\theta\}$ for all distinct $u$ and $v$ among its free variables.

For each $u \in \bar{y}$, let $\sigma_{u}$ be the longest string such that $D_{\sigma}(u)$ occurs in $\theta$, if one exists, and let $\sigma_{u}=\lambda$ otherwise. Since $\mathcal{T}$ is strongly atomic, for each $u \in \bar{y}$ there is an atom $\rho_{u}$ of $\mathcal{T}$ extending $\sigma_{u}$.

Let $u \in \bar{y}$. If $R_{i}(u)$ occurs in $\theta$ for some $i$, then, for the least such $i$, let $t(u)=r_{i}(u)$. If $\neg D_{\tau}(u)$ and $\neg U(u)$ both occur in $\theta$ for some $\tau$ compatible with $\rho_{u}$, then for the shortest such $\tau$, let $t(u)=d_{\tau}(u)$. 
If $P(v, u)$ occurs in $\theta$ for $v \in \bar{x}$, and $t(u)$ has not yet been defined, then let $t(u)$ be the 1-type that includes $U(u)$ and includes $R_{i}(u)$ if and only if $p$ includes $\neg D_{\sigma}(v)$ for all $\sigma \in \mathcal{T}$ of length $i$. Notice that $t(u)$ either is $r_{j}(u)$ for some $j$ or is $s$.

If $P(u, v)$ occurs in $\theta$ for $v \in \bar{x}$, and $t(u)$ has not yet been defined, then let $t(u)$ be the 1-type defined as follows. The type $t(u)$ includes $\neg U(u)$. For each $n$ such that $\neg R_{n}(v) \in p$, it includes $D_{\tau}(u)$ for the unique $\tau \in \mathcal{T}$ of length $n$ that is compatible with $\rho_{u}$. If $R_{n}(v) \in p$, then $t(u)$ includes $\neg D_{\tau}(u)$ for all $\tau$ of length $n$. Notice that $t(u)$ either is $d_{\tau}(u)$ for some $\tau \succcurlyeq \sigma_{u}$ or is $d_{\rho_{u}}(u)$.

If $P(u, v)$ occurs in $\theta$ for some $v \in \bar{y}$ for which we have defined $t(v)$, and $t(u)$ has not yet been defined, then define $t(u)$ as in the previous paragraph, with $t(v)$ in place of $p$.

If $P(v, u)$ occurs in $\theta$ for some $v \in \bar{y}$ for which we have defined $t(v)$, and $t(u)$ has not yet been defined, then we must have defined $t(v)=r_{i}(v)$ for some $i$. In this case, let $t(u)$ be some $d_{\sigma}(u)$ such that $|\sigma|=i$ and $d_{\sigma}(u)$ is consistent with the literals of the form $D_{\tau}(u)$ and $\neg D_{\tau}(u)$ occurring in $\theta$.

If we have not yet defined $t(u)$ for $u \in \bar{y}$, then no $R_{i}(u)$ or $\neg D_{\tau}(u)$ for $\tau$ compatible with $\rho_{u}$ occurs in $\theta$. In this case, if $U(u)$ occurs in $\theta$ then let $t(u)=s$, and otherwise let $t(u)=d_{\rho_{u}}(u)$.

It is straightforward to check that applying the $\mathcal{A}$ operator to $p$, the types $t(u)$ with $u \in \bar{y}$, and $\theta$ yields a type $q$ containing both $p$ and $\theta$, and that there is a sequence of strings $t$ such that $\mathcal{N}(q, t)=q$. Thus $q \in X$.

Applying $\mathrm{EFAE}_{\mathcal{C}}$. By $\mathrm{EFAE}_{\mathcal{C}}$, we have an extension function approximation $g$ for $X$. Recall the type $s$ defined above. Let $i$ be such that $p_{i}=s\left(x_{0}\right)$. Given $\sigma \in \mathcal{T}$, let $j$ be such that $\theta_{j}=D_{\sigma}\left(x_{1}\right) \wedge P\left(x_{1}, x_{0}\right)$. Applying $g$ to $i$ and $j$, we obtain a 2-type $q\left(x_{0}, x_{1}\right)$ of $\Delta$ amalgamating $p_{i}$ and $\theta_{j}$. Note that this type is obtained effectively from $i$ and $j$, as $q \uparrow s=p_{g(i, j, s)} \uparrow s$ for all $s$. Since $\lim _{s} g(i, j, s)$ exists, $q \in X$, so $q\left\lceil x_{1} \in X\right.$. The only 1-types in $X$ are the ones of the form $d_{\rho}, e_{\rho}$, $r_{i}$, or $s$. Since $\neg R_{j}\left(x_{0}\right) \in q$ for all $j$, for each $m$ there must be some $\tau$ of length $m$ such that $D_{\tau}\left(x_{1}\right) \in q$. Thus we must have $q\left\lceil x_{1}=e_{\rho}\right.$ for some atom $\rho$ of $\Delta$. Then $f_{\sigma}=\bigcup_{D_{\tau}\left(x_{1}\right) \in q} \tau$ is an isolated path of $\mathcal{T}$. The function $\sigma \mapsto f_{\sigma}$ exists. Let $\sigma_{0}, \sigma_{1}, \ldots$ be a listing of the elements of $\mathcal{T}$ and let $g_{i}=f_{\sigma_{i}}$. Then $\left\{g_{i}\right\}_{i \in \mathbb{N}}$ is a listing of the isolated paths of $\mathcal{T}$.

Theorems 7.1 and 7.4 together give us the following results.

THEOREM 7.5. (1) Let $\mathcal{C}$ denote the finite full or finite free amalgamation closure conditions. Then $\mathrm{EFAE}_{\mathcal{C}}$ is equivalent to AMT over $\mathrm{RCA}_{0}$.

(2) Let $\mathcal{C}$ be any set of closure conditions that includes the extension condition, in particular the pairwise full, pairwise free, or 1-point full amalgamation closure conditions. Then $\mathrm{EFAE}_{\mathcal{C}}$ implies AMT and is implied by ATT over $\mathrm{RCA}_{0}$.

Assuming AMT does not imply ATT, the second part of the above theorem has a gap, which remains open.

\subsection{Comparing model existence theorems}

As mentioned above, HMT and WMT are each equivalent to the corresponding $\mathrm{EFAE}_{\mathcal{C}}$ principle over $\mathrm{RCA}_{0}$, so Theorem 7.5 gives us the following result.

TheORem 7.6. Both HMT and WMT are equivalent to AMT over $\mathrm{RCA}_{0}$. 
Now consider the statement that every list of types that satisfies the 1-point full amalgamation closure conditions and has an extension function approximation is an enumeration of the type spectrum of some 1-point homogeneous model. By Theorem 6.12, this statement implies $\mathrm{I}_{2}^{0}$ over $\mathrm{RCA}_{0}$. Since 1-PHMT implies this statement, it also implies $\mathrm{I} \Sigma_{2}^{0}$ over $\mathrm{RCA}_{0}$. Since 1-PHMT also implies $\mathrm{EFAE}_{\mathcal{C}}$, where $\mathcal{C}$ denotes the 1-point full amalgamation closure conditions, it follows from Theorem 7.5 that 1-PHMT also implies AMT over $\mathrm{RCA}_{0}$. On the other hand, by Theorem 5.11, under $\mathrm{RCA}_{0}+\mathrm{I} \Sigma_{2}^{0}$, satisfying the 1-point full amalgamation closure conditions implies satisfying the finite full amalgamation closure conditions. Thus we have the following result.

THEOREM 7.7. 1-PHMT is equivalent to $\mathrm{AMT}+\mathrm{I} \Sigma_{2}^{0}$ over $\mathrm{RCA}_{0}$.

The situation for 1-HMT and SMT is not quite as clear. Since they each imply the corresponding $\mathrm{EFAE}_{\mathcal{C}}$ principle over $\mathrm{RCA}_{0}$, Theorem 7.5 gives us the following result.

TheOrem 7.8. Both 1-HMT and SMT imply AMT over $\mathrm{RCA}_{0}$.

By Theorems 6.10 and 7.5, we also have the following result.

THEOREM 7.9. Both 1 -HMT and SMT are provable in $\mathrm{RCA}_{0}+\mathrm{ATT}+\Pi_{1}^{0} \mathrm{GA}$.

Let $\mathcal{P}$ be one of 1 -HMT or SMT, and let $\mathcal{C}_{\mathcal{P}}$ be the set of closure conditions mentioned in the statement of $\mathcal{P}$. Consider the statement that if $X$ is a list of types satisfying $\mathcal{C}_{\mathcal{P}}$ and $X$ has an extension function approximation, then there is a (1-homogeneous, if $\mathcal{P}$ is 1 -HMT) model $\mathcal{A}$ such that $X$ enumerates the type spectrum of $\mathcal{A}$. By Theorem 6.13 (in particular, item $\mathrm{T}$ in the charts justifying that theorem), this statement implies $\mathrm{I} \Sigma_{2}^{0}$ over $\mathrm{RCA}_{0}+\mathrm{B} \Sigma_{2}^{0}$. Since the statement clearly follows from $\mathcal{P}$ over $\mathrm{RCA}_{0}$, we see that $\mathcal{P}$ implies $\mathrm{I}_{2}^{0}$ over $\mathrm{RCA}_{0}+\mathrm{B} \Sigma_{2}^{0}$. But ATT is $\Pi_{1}^{1}$-conservative over $\mathrm{RCA}_{0}+\mathrm{B} \Sigma_{2}^{0}$, as mentioned in Chapter 3, so we have the following result, which in particular implies that Theorem 7.8 cannot be reversed, even over $\mathrm{B} \Sigma_{2}^{0}$.

THEOREM 7.10. Neither 1 -HMT nor SMT are provable in $\mathrm{RCA}_{0}+\mathrm{ATT}+\mathrm{B} \Sigma_{2}^{0}$.

We do not know the exact strengths of 1-HMT and SMT.

\subsection{Computability theoretic equivalences}

Theorem 7.6 has a strong computability theoretic analog. In the computability theoretic setting, where we work over the standard model of first order arithmetic, there is no difference between our versions of HMT. There is also no difference between SMT and WMT, so in this section we refer to this principle as MT. Similarly, in this setting we have only two versions of $\mathrm{EFAE}_{\mathcal{C}}$, one for full amalgamation, which we call $\mathrm{EFAE}_{\mathrm{full}}$, and one for free amalgamation, which we call EFAE free.

We may think of a principle of the form $\forall X[\Theta(X) \rightarrow \exists Y \Psi(X, Y)]$, where $\Theta$ and $\Psi$ are arithmetic, as a problem. An instance of this problem is an $X$ such that $\Theta(X)$ holds and a solution to this instance is a $Y$ such that $\Psi(X, Y)$ holds. For example, an instance of HMT consists of a theory $T$ together with a list of types $X$ of $T$ satisfying the full amalgamation closure conditions, and a solution to this instance is the elementary diagram of a homogeneous model $\mathcal{A}$ of $T$ such that $X$ is an enumeration of $\mathbb{T}(\mathcal{A})$. 
Let $P$ and $Q$ be problems. We say that $P$ is uniformly reducible to $Q$, and write $P \leqslant \mathrm{u} Q$, if there are Turing functionals $\Phi$ and $\Psi$ such that, for every instance $X$ of $P$, the set $\widehat{X}=\Phi^{X}$ is an instance of $Q$, and for every solution $\widehat{Y}$ to $\widehat{X}$, the set $Y=\Psi^{X \oplus \widehat{Y}}$ is a solution to $X$. We say that $P$ and $Q$ are uniformly equivalent, and write $P \equiv_{\mathrm{u}} Q$, if $P \leqslant_{\mathrm{u}} Q$ and $Q \leqslant_{\mathrm{u}} P$. (As noted by Dorais, Dzhafarov, Hirst, Mileti, and Shafer [10], uniform reducibility is equivalent to a special case of the notion of Weihrauch reducibility that has been widely studied in computable analysis. This notion and other related computability theoretic reducibilities between problems, many with strong relations to reverse mathematics, are presented and analyzed in papers such as [10] and Hirschfeldt and Jockusch [21].)

Theorem 7.11. HMT $\equiv_{u} \mathrm{MT} \equiv_{u} \mathrm{AMT}$.

ProOF. It is easy to check that the translations between trees and theories in Chapter 3 are uniform, so AMT $\equiv_{\mathrm{u}}$ ATT. (In this setting, there is no distinction between atomic and strongly atomic trees.)

The proof of Proposition 6.6 shows that EFAE $_{\text {full }} \leqslant \mathrm{u}$ HMT and $\mathrm{EFAE}_{\text {free }} \leqslant_{\mathrm{u}}$ MT. The proofs of Lemma 6.9 and Theorem 6.10 are also both uniform, so in fact $\mathrm{HMT} \equiv_{\mathrm{u}}$ EFAE $_{\text {full }}$ and $\mathrm{MT} \equiv_{\mathrm{u}}$ EFAE $_{\text {free }}$. (These facts can also be obtained from the original proofs of Theorem 6.4 by Goncharov [15] and Peretyat'kin [41].)

The construction of the tree $\mathcal{T}$ from the theory $T$ and list of types $X$ in the proof of Theorem 7.1 is uniform, as is the construction of an extension function approximation for $X$ from an enumeration of the isolated paths of $\mathcal{T}$. Thus ATT $\leqslant_{\mathrm{u}}$ EFAE $_{\text {free. }}$

In the proof of Theorem 7.4, the construction of the theory $\Delta$ and the list of types $X$ from the tree $\mathcal{T}$ is uniform. The construction of a listing of the isolated paths of $\mathcal{T}$ from an extension function approximation for $X$ is also uniform, except for the choice of an $i$ such that $p_{i}=s\left(x_{0}\right)$. We can find such an $i$ if we know an atom $\sigma$ of $\mathcal{T}$, since then, given our listings $\sigma_{0}, \sigma_{1}, \ldots$ of $\mathcal{T}$ and $t_{0}, t_{1}, \ldots$ of $B$ (where $B$ is as in the proof of Theorem 7.4), we can fix $m$ and $i$ such that $\sigma_{m}=\sigma$ and $t_{i}=s$, and have that $p_{\langle 1,\langle i, 0, m\rangle\rangle}=s_{x_{0}}$. So if we let $\widehat{\mathcal{T}}$ be the tree consisting of all strings of the form $0^{n}$ and all strings of the form $1 \tau$ for $\tau \in \mathcal{T}$, and apply our construction to $\widehat{\mathcal{T}}$ instead of $T$, we get a fully uniform construction. From a listing of the isolated paths of $\widehat{\mathcal{T}}$, we can uniformly obtain one of the isolated paths of $\mathcal{T}$, so EFAE $_{\text {full }} \leqslant$ uTT.

The above results, together with the obvious fact that $\mathrm{EFAE}_{\mathrm{full}} \leqslant \mathrm{u} \mathrm{EFAE}_{\text {free}}$, yield the theorem.

For a problem $P$, a degree a is $P$-bounding if every computable instance of $P$ has an a-computable solution. (The AMT-bounding degrees have been referred to in the literature as atomic bounding or prime bounding.) More generally, a is $P$-bounding relative to $\mathbf{b}$ if every $\mathbf{b}$-computable instance of $P$ has an a-computable solution.

Corollary 7.12. For any degree $\mathbf{b}$, the HMT-bounding degrees relative to $\mathbf{b}$, the MT-bounding degrees relative to $\mathbf{b}$, and the AMT-bounding degrees relative to b all coincide. 



\section{CHAPTER 8}

\section{Open Questions}

In this chapter, we gather some open questions raised in previous chapters.

As mentioned in Chapter $3, \Pi_{1}^{0} \mathrm{G}$ implies AMT $+\Pi_{1}^{0} \mathrm{GA}$, but, although $\Pi_{1}^{0} \mathrm{GA}$ can be seen as an attempt to capture the first order difference between AMT and $\Pi_{1}^{0} \mathrm{G}$, the reverse implication remains open.

Question 8.1. Does AMT $+\Pi_{1}^{0} \mathrm{GA}$ imply $\Pi_{1}^{0} \mathrm{G}$ over $\mathrm{RCA}_{0}$ ?

In connection with both this question and the following one, recall from Corollary 3.4 that AMT does not imply $\Pi_{1}^{0} \mathrm{GA}$.

As noted following Proposition 3.6, AMT is equivalent to the statement that if $\mathcal{T}$ is a strongly atomic extendible tree, then there is a listing of the isolated paths of $\mathcal{T}$. Weakening the hypothesis of $\mathcal{T}$ being strongly atomic to that of $\mathcal{T}$ being atomic yields ATT. ATT implies AMT, and $\Pi_{1}^{0} \mathrm{G}$ implies ATT. Furthermore, as noted following the definition of ATT in Chapter 3, AMT implies ATT over B $\Sigma_{2}^{0}$.

Question 8.2. Does AMT imply ATT over $\mathrm{RCA}_{0}$ ? Does AMT $+\Pi_{1}^{0} \mathrm{GA}$ imply ATT over $\mathrm{RCA}_{0}$ ? What if we replace ATT by the principle $\mathrm{ATT}^{+}$defined at the end of Appendix B below?

Note that a positive answer to Question 8.1 implies a positive answer to the second part of Question 8.2.

By Proposition 4.5 , it is provable in $\mathrm{RCA}_{0}$ that every saturated model is 1homogeneous, and that every strongly saturated model is homogeneous. As noted following that proposition, the statement that every saturated model is homogeneous is provable in both $\mathrm{B} \Sigma_{2}^{0}$ and $\mathrm{WKL}_{0}$ (and hence does not imply $\mathrm{B} \Sigma_{2}^{0}$ ).

Question 8.3. What is the exact strength of the statement that every saturated model is homogeneous?

Proposition 4.6 says that the statement that every atomic model is strongly 1-homogeneous is equivalent to $\mathrm{ACA}_{0}$. Combining Proposition 4.5 with the fact that $\mathrm{ACA}_{0}$ suffices to show that all of our definitions of homogeneity are equivalent, we see that $\mathrm{ACA}_{0}$ proves that every prime model is strongly 1-homogeneous, and so is every saturated model.

QUESTION 8.4. What are the exact strengths of the statements that every prime model is strongly 1-homogeneous, and that every (strongly) saturated model is strongly 1-homogeneous?

As noted in Section 6.1, the statement that if $X$ has a weak extension function approximation then it has an extension function approximation follows from $\mathrm{I} \Sigma_{2}^{0}$, and Theorem 7.1 implies that it also follows from ATT (assuming $X$ in fact satisfies the extension condition). 
Question 8.5. What more can be said about the strength of the above statement? What about the version of Theorem 6.10 where extension function approximations are replaced by weak extension function approximations?

Recall that, in Table 6.1 , a 3 means that the principle is provable from $\Pi_{1}^{0} \mathrm{GA}$ over $\mathrm{RCA}_{0}$ and is equivalent to $\mathrm{I}_{2}^{0}$ over $\mathrm{RCA}_{0}+\mathrm{B} \Sigma_{2}^{0}$; a 4 means that the principle is provable from $\Pi_{1}^{0} \mathrm{GA}$ over $\mathrm{RCA}_{0}$, but its strength is otherwise unknown; and a 5 means that the principle is provable from $\mathrm{I} \Sigma_{2}^{0}$ over $\mathrm{RCA}_{0}$, but its strength is otherwise unknown.

Question 8.6. Are the principles marked with a 3 in Table 6.1 equivalent to each other? Are they equivalent to $\Pi_{1}^{0} \mathrm{GA}$ ? What more can be said about the items marked 4 or 5 in Table 6.1 ? Are they equivalent to $\Pi_{1}^{0} \mathrm{GA}$ ?

Theorem 7.5 shows that if $\mathcal{C}$ denotes the pairwise full, pairwise free, or 1point full amalgamation closure conditions, or indeed any set of closure conditions containing the extension condition, then $\mathrm{EFAE}_{\mathcal{C}}$ implies AMT and is implied by ATT.

QUESTION 8.7. What is the exact strength of $\mathrm{EFAE}_{\mathcal{C}}$ in the above cases?

Note that if the answer to the first part of Question 8.2 is positive then Theorem 7.5 answers this question.

By Theorem 7.8, both 1-HMT and SMT imply AMT. By Theorems 7.9 and 7.10 , both of these principles are implied by ATT $+\Pi_{1}^{0} \mathrm{GA}$, but not by ATT $+\mathrm{B} \Sigma_{2}^{0}$.

QUESTION 8.8. What are the exact strengths of 1-HMT and SMT?

In Appendix B below, we define the principle FATT, which follows from $\mathrm{B} \Sigma_{2}^{0}$ and from ATT, but is not provable in $\mathrm{RCA}_{0}$.

Question 8.9. Does $\Pi_{1}^{0}$ GA imply FATT over $\mathrm{RCA}_{0}$ ? 


\section{APPENDIX A}

\section{Approximating Generics}

We can define analogs of $\Pi_{1}^{0} \mathrm{G}$ for higher levels of the arithmetic hierarchy. As in the $\Pi_{1}^{0}$ case, when we say that $D$ is a $\Pi_{n}^{0}$ set of strings, we mean that $D$ is a $\Pi_{n}^{0}$ predicate on $2^{<\mathbb{N}}$, and write $\sigma \in D$ to mean that $D$ holds of $\sigma$.

$\Pi_{\mathbf{n}}^{0} \mathbf{G}$ : For any uniformly $\Pi_{n}^{0}$ collection of sets of strings $D_{0}, D_{1}, \ldots$, each of which is dense in $2^{<\mathbb{N}}$, there is a $G \in 2^{\mathbb{N}}$ such that $(\forall i)(\exists m)\left[G\left\lceil m \in D_{i}\right]\right.$.

In Chapter 3, we introduced the $\Pi_{1}^{0}$-generic approximation principle $\Pi_{1}^{0} \mathrm{GA}$, and showed that it is equivalent to $\mathrm{I} \Sigma_{2}^{0}$ over $\mathrm{B} \Sigma_{2}^{0}$. In the spirit of the strong form of the limit lemma, we have the following analogous generic approximation principles. (We use $\left(\forall^{\infty} x\right)$ as an abbreviation of $(\exists y)(\forall x>y)$.)

$\Pi_{\mathbf{n}}^{\mathbf{0}} \mathbf{G A}$ : For any uniformly $\Pi_{n}^{0}$ collection of sets of strings $D_{0}, D_{1}, \ldots$, each of which is dense in $2^{<\mathbb{N}}$, there are $g_{k_{0}, \ldots, k_{n-1}} \in 2^{\mathbb{N}}$ for $k_{0}, \ldots, k_{n-1} \in \mathbb{N}$ such that

$$
(\forall i)\left(\exists \sigma \in D_{i}\right)\left(\forall^{\infty} k_{0}\right) \cdots\left(\forall^{\infty} k_{n-1}\right)\left[g_{k_{0}, \ldots, k_{n-1}} \succ \sigma\right] .
$$

Clearly, $\Pi_{n}^{0} \mathrm{GA}$ follows from $\Pi_{n}^{0} \mathrm{G}$. Let $\mathcal{M}$ be a countable model of $\mathrm{RCA}_{0}$. For a subset $G$ of the domain of $\mathcal{M}$, let $\mathcal{M}[G]$ be the structure obtained by adding to the second order part of $\mathcal{M}$ all sets definable over $\mathcal{M}$ by $\Delta_{1}^{0}$ formulas with $G$ as an added set parameter. If $G$ is Cohen 1-generic, then adding $G$ to $\mathcal{M}$ preserves $\mathrm{I} \Sigma_{1}^{0}$, which implies that $\mathcal{M}[G]$ is a model of $\mathrm{RCA}_{0}$. (See [47]; for more on the general structure of arguments establishing conservativity results of the kind we are discussing, see $[\mathbf{2 0}, \mathbf{2 2}, \mathbf{4 7}]$.) It is easy to see that, if $G$ is sufficiently Cohen generic, then $\mathcal{M}[G]$ contains solutions to all instances of $\Pi_{n}^{0} \mathrm{G}$ in $\mathcal{M}$. By iterating this construction, we obtain an extension $\mathcal{N}$ of $\mathcal{M}$ that is a model of $\mathrm{RCA}_{0}+\Pi_{n}^{0} \mathrm{G}$, which is enough to conclude that $\Pi_{n}^{0} \mathrm{G}$ is $\Pi_{1}^{1}$-conservative over $\mathrm{RCA}_{0}$. In particular, $\Pi_{n}^{0} \mathrm{G}$ (and hence $\Pi_{n}^{0} \mathrm{GA}$ ) does not imply I $\Sigma_{n+1}^{0}$, even over I $\Sigma_{n}^{0}$. (In fact, the same argument as in $[\mathbf{2 3}]$ for $\Pi_{1}^{0} \mathrm{G}$ shows that $\Pi_{n}^{0} \mathrm{G}$ is restricted $\Pi_{2}^{1}$-conservative over $\mathrm{RCA}_{0}$.)

We have the following analogs to Theorems 3.2 and 3.3 .

Theorem A.1. $\mathrm{RCA}_{0}+\mathrm{I} \Sigma_{n+1}^{0} \vdash \Pi_{n}^{0} \mathrm{GA}$.

Proof. Let $D_{0}, D_{1}, \ldots$ be uniformly $\Pi_{n}^{0}$ sets of strings, each of which is dense in $2^{<\mathbb{N}}$. Let $\Theta$ be a $\Delta_{0}^{0}$ predicate such that $\sigma \in D_{i}$ if and only if

$$
\left(\forall x_{0}\right)\left(\exists x_{1}\right) \cdots\left(Q x_{n-1}\right) \Theta\left(i, \sigma, x_{0}, \ldots, x_{n-1}\right),
$$

where $Q$ is either $\exists$ or $\forall$, depending on the parity of $n$. Let

$$
\begin{aligned}
& D_{i}\left[k_{0}, \ldots, k_{n-1}\right]=\left\{\sigma:|\sigma|>k_{n-1} \vee\right. \\
&\left.\left(\forall x_{0}<k_{0}\right)\left(\exists x_{1}<k_{1}\right) \cdots\left(Q x_{n-1}<k_{n-1}\right) \Theta\left(i, \sigma, x_{0}, \ldots, x_{n-1}\right)\right\} .
\end{aligned}
$$


Let $\leqslant$ ll be the length-lexicographic order on $2^{<\mathbb{N}}$. For each tuple $\left(k_{0}, \ldots, k_{n-1}\right)$, define $\sigma_{k_{0}, \ldots, k_{n-1}, i}$ for $i \leqslant k_{n-1}$ by recursion as follows. Let $\sigma_{k_{0}, \ldots, k_{n-1}, 0}$ be the $\leqslant 1^{-}$ least string in $D_{0}\left[k_{0}, \ldots, k_{n-1}\right]$. For $i>0$, given $\sigma_{k_{0}, \ldots, k_{n-1}, i-1}$, let $\sigma_{k_{0}, \ldots, k_{n-1}, i}$ be the $\leqslant 11$-least extension of $\sigma_{k_{0}, \ldots, k_{n-1}, i-1}$ in $D_{i}\left[k_{0}, \ldots, k_{n-1}\right]$. Let $g_{k_{0}, \ldots, k_{n-1}}=$ $\sigma_{k_{0}, \ldots, k_{n-1}, k_{n-1}} 0^{\mathbb{N}}$.

Let $\varphi(i, \sigma, \tau)$ be a $\Pi_{n}^{0}$ formula stating that $\tau \succcurlyeq \sigma$ and $\tau \in D_{i}$. Let $\psi(i, \sigma, \tau)$ be a formula stating that $\varphi(i, \sigma, \tau)$ and $\neg \varphi(i, \sigma, \rho)$ for all $\rho<_{11} \tau$. Note that for each $i$ and $\sigma$ there is a $\tau$ such that $\psi(i, \sigma, \tau)$, by $\Pi_{n}^{0}$-induction. Consider the statement

$$
\left(\exists\left\langle\sigma_{0}, \ldots, \sigma_{i}\right\rangle\right)\left[\psi\left(0, \lambda, \sigma_{0}\right) \wedge(\forall j<i) \psi\left(j+1, \sigma_{j}, \sigma_{j+1}\right)\right] .
$$

This statement is $\Sigma_{n+1}^{0}$, and if it holds of $i$, then it holds of $i+1$, so by $\Sigma_{n+1^{-}}^{0}$ induction, it holds of all $i$. Fix $i$ and let $\sigma_{0}, \ldots, \sigma_{i}$ be as in the statement. We claim that

$$
\left(\forall^{\infty} k_{0}\right) \cdots\left(\forall^{\infty} k_{n-1}\right)\left[g_{k_{0}, \ldots, k_{n-1}} \succ \sigma_{i}\right]
$$

which is enough to establish the theorem, as $\sigma_{i} \in D_{i}$.

For each $j \leqslant i$ and each $\rho<_{11} \sigma_{j}$ such that $\rho \succcurlyeq \sigma_{j-1}$ (or $j=0$ ), there is an $m_{\rho, j}$ such that

$$
\neg\left(\exists x_{1}\right) \cdots\left(Q x_{n-1}\right) \Theta\left(j, \rho, m_{\rho, j}, x_{1}, \ldots, x_{n-1}\right),
$$

so by $\Pi_{n-1}^{0}$-bounding, there is an $m_{0}$ bounding such an $m_{j, \rho}$ for each such $j$ and $\rho$. Fix $k_{0}>m_{0}$. It is enough to show that

$$
\left(\forall^{\infty} k_{1}\right) \cdots\left(\forall^{\infty} k_{n-1}\right)\left[g_{k_{0}, \ldots, k_{n-1}} \succ \sigma_{i}\right]
$$

For each $j \leqslant i$,

$$
\left(\forall x_{0}<k_{0}\right)\left(\exists x_{1}\right)\left(\forall x_{2}\right) \cdots\left(Q x_{n-1}\right) \Theta\left(j, \sigma_{j}, x_{0}, x_{1}, \ldots, x_{n-1}\right),
$$

so by $\Pi_{n-2}^{0}$-bounding, there is an $m_{1}$ such that for all $j \leqslant i$,

$$
\left(\forall x_{0}<k_{0}\right)\left(\exists x_{1}<m_{1}\right)\left(\forall x_{2}\right) \cdots\left(Q x_{n-1}\right) \Theta\left(j, \sigma_{j}, x_{0}, x_{1}, \ldots, x_{n-1}\right) .
$$

Fix $k_{1} \geqslant m_{1}$. It is enough to show that

$$
\left(\forall^{\infty} k_{2}\right) \cdots\left(\forall^{\infty} k_{n-1}\right)\left[g_{k_{0}, \ldots, k_{n-1}} \succ \sigma_{i}\right] .
$$

For each $j \leqslant i$ and each $\rho<_{11} \sigma_{j}$ such that $\rho \succcurlyeq \sigma_{j-1}$ (or $j=0$ ),

$$
\neg\left(\exists x_{1}<k_{1}\right)\left(\forall x_{2}\right)\left(\exists x_{3}\right) \cdots\left(Q x_{n-1}\right) \Theta\left(j, \rho, m_{j, \rho}, x_{1}, \ldots, x_{n-1}\right),
$$

where $m_{j, \rho}$ is as above, so for each $x_{1}<k_{1}$, there is an $m_{j, \rho, x_{1}}$ such that

$$
\neg\left(\forall x_{2}<m_{j, \rho, x_{1}}\right)\left(\exists x_{3}\right) \cdots\left(Q x_{n-1}\right) \Theta\left(j, \rho, m_{j, \rho}, x_{1}, \ldots, x_{n-1}\right) .
$$

Let $m_{2}$ bound all such $m_{j, \rho, x_{1}}$. Fix $k_{2}>m_{2}$. It is enough to show that

$$
\left(\forall^{\infty} k_{3}\right) \cdots\left(\forall^{\infty} k_{n-1}\right)\left[g_{k_{0}, \ldots, k_{n-1}} \succ \sigma_{i}\right] \text {. }
$$

We continue in this manner until we have defined $k_{0}, \ldots, k_{n-1}$, and established that it is enough to show that $g_{k_{0}, \ldots, k_{n-1}} \succ \sigma_{i}$. (We make sure to have $k_{n-1} \geqslant i$.) Our definition ensures that for each $j \leqslant i$, we have $\sigma_{j} \in D_{j}\left[k_{0}, \ldots, k_{n-1}\right]$ and $\rho \notin D_{j}\left[k_{0}, \ldots, k_{n-1}\right]$ for all $\rho<_{11} \sigma_{j}$ such that $\rho \succcurlyeq \sigma_{j-1}$ (or $j=0$ ), whence $\sigma_{k_{0}, \ldots, k_{n-1}, j}=\sigma_{j}$. Thus $g_{k_{0}, \ldots, k_{n-1}} \succ \sigma_{i}$, as desired.

Theorem A.2. $\mathrm{RCA}_{0}+\mathrm{B} \Sigma_{n+1}^{0}+\Pi_{n}^{0} \mathrm{GA} \vdash \mathrm{I} \Sigma_{n+1}^{0}$, and hence $\mathrm{RCA}_{0}+\mathrm{B} \Sigma_{n+1}^{0} \vdash$ $\Pi_{n}^{0} \mathrm{GA} \leftrightarrow \mathrm{I} \Sigma_{n+1}^{0}$. 
Proof. Fix a model of $\mathrm{RCA}_{0}+\mathrm{B} \Sigma_{n+1}^{0}+\Pi_{n}^{0} \mathrm{GA}$ with first order part $M$ and second order part $\mathcal{S}$. Suppose for the sake of a contradiction that $I \Sigma_{n+1}^{0}$ fails in this model. As in the proof of Theorem 3.3, there is a $\Sigma_{n+1}^{0} \operatorname{cut} \mathcal{I}$ in $M$. As in that proof, let $D_{i}$ be the set of all $\sigma \in 2^{<\mathbb{N}}$ such that, thinking of $\sigma$ as a finite set, the last two elements of $\sigma$, in order, are $w$ and $\langle i, x, y\rangle$, and

$$
\left(\exists y^{\prime}<y\right)(\forall z)\left[\varphi\left(x, y^{\prime}, z\right) \wedge\left(\forall y^{\prime} \leqslant w\right)\left(\exists z^{\prime}<y\right) \neg \varphi\left(x, y^{\prime}, z^{\prime}\right)\right] .
$$

The $D_{i}$ are uniformly $\Pi_{n}^{0}$. There is no bound on the least witnesses $y$ such that $(\forall z) \varphi(x, y, z)$ for $x \in \mathcal{I}$, as otherwise $\mathcal{I}$ would be a $\Pi_{n}^{0}$ cut, which is not possible since II ${ }_{n}^{0}$ holds in our model. Now the same argument as in the proof of Theorem 3.3 shows that each $D_{i}$ is dense. Let

Let $g_{k_{0}, \ldots, k_{n-1}}$ be as in $\Pi_{n}^{0} \mathrm{GA}$ and let $a$ be any number above all those in $\mathcal{I}$.

$$
\begin{aligned}
E_{i}=\left\{\langle\sigma, m\rangle: \sigma \in D_{i} \wedge\right. & \\
& \left.\left(\forall k_{0}>m\right)\left(\exists^{\infty} k_{1}\right)\left(\forall^{\infty} k_{2}\right) \cdots\left(Q^{\infty} k_{n-1}\right) g_{k_{0}, \ldots, k_{n-1}} \succ \sigma\right\},
\end{aligned}
$$

where $Q$ is either $\exists$ or $\forall$ depending on the parity of $n$. Note that the $E_{i}$ are uniformly $\Pi_{n}^{0}$. For each $i<a$ there is a $\langle\sigma, m\rangle \in E_{i}$, so by the finite axiom of choice for $\Pi_{n}^{0}$ properties, which follows from $\mathrm{B} \Sigma_{n+1}^{0}$, there is a function $f$ with domain $a$ that is $M$-finite and such that for each $i<a$ there is a $\langle\sigma, m\rangle \in E_{i}$ with the last element of $\sigma$ of the form $\langle i, f(i), y\rangle$. Since $\langle\sigma, m\rangle \in E_{i} \Rightarrow \sigma \in D_{i}$, every $f(i)$ is in $\mathcal{I}$.

We claim that $f$ is one-to-one, which gives us a contradiction as in the proof of Theorem 3.3. So assume for a contradiction that $f(i)=f(j)=x$ for $i \neq j$. Then there are $\langle\sigma, m\rangle \in E_{i}$ and $\left\langle\sigma^{\prime}, m^{\prime}\right\rangle \in E_{j}$, and there are $v, w, y_{i}, y_{j}$, such that the next to last and last elements of $\sigma$ are $v$ and $\left\langle i, x, y_{i}\right\rangle$, and the next to last and last elements of $\sigma^{\prime}$ are $w$ and $\left\langle j, x, y_{j}\right\rangle$. Let $l=\max \left(|\sigma|,\left|\sigma^{\prime}\right|\right)$, and let $\tau \in D_{l}$ be such that

$$
\left(\forall^{\infty} k_{0}\right)\left(\forall^{\infty} k_{1}\right) \cdots\left(\forall^{\infty} k_{n-1}\right) g_{k_{0}, \ldots, k_{n-1}} \succ \tau .
$$

Note that $|\tau|>l$. Let $m_{0}$ be such that

$$
\left(\forall k_{0}>m_{0}\right)\left(\forall^{\infty} k_{1}\right) \cdots\left(\forall^{\infty} k_{n-1}\right) g_{k_{0}, \ldots, k_{n-1}} \succ \tau
$$

and let $k_{0}>m, m_{0}$. Let $m_{1}$ be such that

$$
\left(\forall k_{1}>m_{1}\right)\left(\forall^{\infty} k_{2}\right) \cdots\left(\forall^{\infty} k_{n-1}\right) g_{k_{0}, \ldots, k_{n-1}} \succ \tau,
$$

and let $k_{1}>m_{1}$ be such that

$$
\left(\forall^{\infty} k_{2}\right)\left(\exists^{\infty} k_{3}\right) \cdots\left(Q^{\infty} k_{n-1}\right) g_{k_{0}, \ldots, k_{n-1}} \succ \sigma .
$$

Let $m_{2}$ be such that

$$
\left(\forall k_{2}>m_{2}\right)\left(\forall^{\infty} k_{3}\right) \cdots\left(\forall^{\infty} k_{n-1}\right) g_{k_{0}, \ldots, k_{n-1}} \succ \tau,
$$

and let $k_{2}>m_{2}$ be such that

$$
\left(\exists^{\infty} k_{3}\right)\left(\forall^{\infty} k_{3}\right) \cdots\left(Q^{\infty} k_{n-1}\right) g_{k_{0}, \ldots, k_{n-1}} \succ \sigma .
$$

Keep defining $m_{l}$ and $k_{l}$ this way, to obtain $k_{0}, \ldots, k_{n-1}$. Then $g_{k_{0}, \ldots, k_{n-1}} \succ \tau$ and $g_{k_{0}, \ldots, k_{n-1}} \succ \sigma$, so $\sigma \prec \tau$. The same argument shows that $\sigma^{\prime} \prec \tau$. Thus $\sigma$ and $\sigma^{\prime}$ are compatible. We cannot have $\sigma=\sigma^{\prime}$, since $\sigma$ and $\sigma^{\prime}$ have different last elements, so, without loss of generality, we may assume that $\sigma \prec \sigma^{\prime}$ and so $v<\left\langle i, x, y_{i}\right\rangle \leqslant w<\left\langle j, x, y_{j}\right\rangle$. We now have a contradiction, as the definitions of $D_{i}$ and $D_{j}$ then say that $\left(\exists y^{\prime}<y_{i}\right)(\forall z) \varphi\left(x, y^{\prime}, z\right)$ and $\left(\forall y^{\prime} \leqslant w\right)\left(\exists z^{\prime}<y\right) \neg \varphi\left(x, y^{\prime}, z^{\prime}\right)$, respectively, but $y_{i} \leqslant w$. 



\section{APPENDIX B}

\section{Atomic Trees}

In this appendix, we discuss a couple of results on atomic trees mentioned above. We begin with the following fact.

Proposition B.1. The statement that every atomic extendible tree is strongly atomic is equivalent to $\mathrm{B} \Sigma_{2}^{0}$ over $\mathrm{RCA}_{0}$.

Proof. Since being an atom of a tree is a $\Pi_{1}^{0}$ property, the statement follows from the finite axiom of choice for $\Pi_{1}^{0}$ properties. In the other direction, we assume that every atomic extendible tree is strongly atomic and argue in $\mathrm{RCA}_{0}$ as follows. Let $P$ be a $\Pi_{1}^{0}$ property and $m$ be such that $(\forall i<m)(\exists u) P(i, u)$. Let $R$ be a $\Delta_{1}^{0}$ property such that $P(x, y)$ if and only if $(\forall z) R(x, y, z)$, and for $i<m$, let $w(i, s)$ be the least $u$ such that $(\forall z<s) R(i, u, s)$. We define a tree $\mathcal{T}$ in stages. At stage 0 put into $\mathcal{T}[0]$ all strings of the forms $0^{i}$ and $0^{i} 1$ for $i<m$. At stage $s+1$, for each $i<m$ and each leaf $\sigma$ of $\mathcal{T}[s]$ extending $0^{i} 1$, if $w(i, s+1)=w(i, s)$ then put $\sigma 0$ into $\mathcal{T}[s+1]$, and otherwise put both $\sigma 0$ and $\sigma 1$ into $\mathcal{T}[s+1]$.

It is easy to check that $\mathcal{T}$ is a well-defined extendible tree. Let $\tau \in \mathcal{T}$. By extending $\tau$ if needed, we may assume that $\tau$ extends $0^{i} 1$ for some $i<m$. Since $P(i, u)$ holds for some $u$, there is an $s \geqslant|\tau|$ such that $w(i, t)=w(i, s)$ for all $t>s$. Let $\sigma \succ \tau$ be a leaf of $\mathcal{T}[s]$. Then $\sigma$ is an atom of $\mathcal{T}$ extending $\tau$. Thus $\mathcal{T}$ is atomic, and hence by hypothesis $\mathcal{T}$ is strongly atomic. Let $\tau_{0}, \ldots, \tau_{m-1}$ be atoms of $\mathcal{T}$ extending $01,001, \ldots, 0^{m-1} 1$, respectively, and let $s$ be greater than the length of each $\tau_{i}$. Then $w(i, t)=w(i, s)$ for all $t>s$, so $P(i, w(i, s))$ holds for all $i<m$. Letting $v$ be greater than all $w(i, s)$ for $i<m$, we have $(\forall i<m)(\exists u<v) P(i, u)$. Thus $\mathrm{B}_{1}^{0}$ holds. 3.

Next, we define the "miniaturization" of ATT discussed at the end of Chapter

FATT (Finite Atomic Tree Theorem): For each atomic extendible tree $\mathcal{T}$ and each finite set of nodes $\sigma_{0}, \ldots, \sigma_{k} \in \mathcal{T}$, there is a set of isolated paths $P_{0}, \ldots, P_{k}$ such that $P_{i}$ extends $\sigma_{i}$.

It is easy to see that FATT follows from B $\Sigma_{2}^{0}$. It also follows from ATT, and hence from $\Pi_{1}^{0} \mathrm{G}$, so it is strictly weaker than $\mathrm{B} \Sigma_{2}^{0}$. We do not know whether FATT follows from $\Pi_{1}^{0} \mathrm{GA}$, but we can show it is not provable in $\mathrm{RCA}_{0}$.

Theorem B.2. RCA $\nvdash$ FATT.

Proof. Define a $k$-path list to be a sequence $L=\left(P_{0}, \ldots, P_{k}\right) \in\left(2^{\mathbb{N}}\right)^{k+1}$ such that $P_{i}$ extends $0^{i} 1$. We write $L(i)$ for $P_{i}$. We assume that $\mathrm{B} \Sigma_{2}^{0}$ fails and argue in $\mathrm{RCA}_{0}$ to build a computable (i.e., $\Delta_{1}^{0}$-definable) atomic extendible tree $\mathcal{T}$ such that, for some $k$, we have $0^{i} 1 \in \mathcal{T}$ for all $i \leqslant k$, but there is no computable $k$-path list $L$ such that $L(i)$ is an isolated path of $\mathcal{T}$ for all $i \leqslant k$. The reason such a 
construction suffices to establish the theorem is the following: Let $\mathcal{N}$ be a model of $\Sigma_{1}^{0}$-PA (i.e., Peano Arithmetic with induction restricted to $\Sigma_{1}^{0}$ formulas) in which $\mathrm{B} \Sigma_{2}^{0}$ fails. Let $\mathcal{M}$ be the structure in the language of second order arithmetic with first order part $\mathcal{N}$ and second order part consisting of the subsets of the domain of $\mathcal{N}$ that are $\Delta_{1}^{0}$-definable over $\mathcal{N}$. Then $\mathcal{M}$ is a model of $\mathrm{RCA}_{0}$ (see [47]), and our construction shows that $\mathcal{M}$ is not a model of FATT.

As shown by Hirst [24], $\mathrm{B} \Sigma_{2}^{0}$ is equivalent over $\mathrm{RCA}_{0}$ to $\mathrm{RT}_{<\infty}^{1}$, the statement that for each $k$ and each partition $A_{0}, \ldots, A_{k}$ of $\mathbb{N}$, some $A_{i}$ is infinite. Thus we may assume there is a $k \in \mathbb{N}$ and a partition $A_{0}, \ldots, A_{k}$ of $\mathbb{N}$ such that each $A_{k}$ is bounded.

Let $L_{0}, L_{1}, \ldots$ list all partial computable $k$-path lists (i.e., partial computable sets that could be extended to $k$-path lists). We construct $\mathcal{T}$ to ensure that if $L_{j}$ is total and $j \in A_{i}$, then $L_{j}(i)$ is not isolated. Our construction is a version of the one used by Goncharov and Nurtazin [16] to build an atomic extendible tree for which there is no computable listing of the isolated paths.

Begin by putting $0^{i} 1$ in $\mathcal{T}$ for all $i \leqslant k$. Every other node in $\mathcal{T}$ extends one of these nodes, so it suffices to describe how $\mathcal{T}$ is built above a fixed $0^{i} 1$ with $i \leqslant k$. We proceed in stages. At each stage, certain strings will be declared to be active. The construction will ensure that if $\sigma$ is active at the end of stage $s$, then there is an $n$ such that the extensions of $\sigma$ put into $\mathcal{T}$ by the end of stage $s$ are exactly those of the form $\sigma 0^{j}$ for $j \leqslant n$.

At stage 0 , declare $0^{i} 1$ to be active.

At stage $s+1$, proceed as follows for each active $\sigma$. Let $n$ be such that the extensions of $\sigma$ put into $\mathcal{T}$ so far are exactly those of the form $\sigma 0^{j}$ for $j \leqslant n$. If by this stage we have not yet found a $j \in A_{i}$ such that $\sigma \prec L_{j}(i)$, then put $\sigma 0^{j+1}$ into $\mathcal{T}$. Otherwise, put both $\sigma 0^{j+1}$ and $\sigma 0^{j} 1$ into $\mathcal{T}$, declare both these strings to be active, and declare $\sigma$ to no longer be active.

It is clear that $\mathcal{T}$ is an extendible tree. Now suppose that $j \in A_{i}$ and $L_{j}(i)$ is an infinite path of $\mathcal{T}$. Let $S$ be the set of all $\sigma \prec L_{j}(i)$ that are active at some point in the construction. If $\sigma \in S$ then, at some point in the construction, $\sigma$ is declared to no longer be active, and strings $\sigma 0^{j+1}$ and $\sigma 0^{j} 1$ are declared to be active. These strings are the only extensions of $\sigma$ of length $|\sigma|+j+1$ in $\mathcal{T}$, so $L_{j}(i)$ must extend one of them. Thus we see that $S$ is infinite, which implies that $L_{j}(i)$ is not isolated, since for every $\tau l \in S$ other than $0^{i} 1$, we also have $\tau(1-l) \in \mathcal{T}$. So we are left with showing that $\mathcal{T}$ is atomic.

Fix a $\rho \in \mathcal{T}$. We may assume that $\rho$ extends some $0^{i} 1$. Let $P$ be any path of $\mathcal{T}$ extending $\rho$ (say the leftmost one). If $P$ is isolated then we are done, so assume otherwise. Then $P$ must go through infinitely many nodes that are active at some point in the construction, and we can find a sequence $\rho \prec \sigma_{0} \prec \sigma_{1} \prec \cdots$ of such nodes. Each $\sigma_{l}$ is of the form $\tau_{l} j_{l}$ for some $\tau_{l}$ and $j_{l} \leqslant 1$. If $\tau_{l}\left(1-j_{l}\right)$ is permanently active then there is a unique path of $\mathcal{T}$ extending $\tau_{l}\left(1-j_{l}\right)$. Since $\tau_{l}\left(1-j_{l}\right)$ extends $\rho$, in this case we are done. So assume that for all $l$, the node $\tau_{l}\left(1-j_{l}\right)$ is not permanently active. Then there is an $n_{l} \in A_{i}$ such that $\tau_{l}\left(1-j_{l}\right) \in L_{n_{l}}(i)$. But if $l<l^{\prime}$ then $\tau_{l}\left(1-j_{l}\right)$ and $\tau_{l^{\prime}}\left(1-j_{l^{\prime}}\right)$ are incompatible, so $n_{l} \neq n_{l^{\prime}}$. Thus we get a computable injective map from $\mathbb{N}$ into the bounded set $A_{i}$, which is a contradiction. 
The statement of FATT suggests the idea of strengthening the hypothesis of ATT by replacing it with the conclusion of FATT, yielding a principle intermediate between ATT and AMT (see Question 8.2 above):

$\mathbf{A T T}^{+}:$Let $\mathcal{T}$ be an extendible tree with the property that for each finite set of nodes $\sigma_{0}, \ldots, \sigma_{k} \in \mathcal{T}$, there is a set of isolated paths $P_{0}, \ldots, P_{k}$ such that $P_{i}$ extends $\sigma_{i}$. Then there is a listing of the isolated paths of $\mathcal{T}$. 



\section{APPENDIX C}

\section{Saturated Models}

Recall that $\mathcal{A}$ is saturated if for all $\bar{a} \subseteq A$, each complete type of $\operatorname{Th}(\mathcal{A}, \bar{a})$ is realized in $\mathcal{A}$; and strongly saturated if for all $\bar{a} \subseteq A$, each partial type of $\operatorname{Th}(\mathcal{A}, \bar{a})$ is realized in $\mathcal{A}$. In this appendix, we prove the following result, mentioned in Section 2.2.

THEOREM C.1. The statement that every saturated model is strongly saturated is equivalent to $\mathrm{WKL}_{0}$ over $\mathrm{RCA}_{0}$.

Proof. To show that the statement holds in $\mathrm{WKL}_{0}$, it is enough to fix $\bar{a} \subseteq A$ and show in $\mathrm{WKL}_{0}$ that every partial type of $\operatorname{Th}(\mathcal{A}, \bar{a})$ is contained in a complete type of $\operatorname{Th}(\mathcal{A}, \bar{a})$. Let $p(\bar{x})$ be a partial $n$-type of $\operatorname{Th}(\mathcal{A}, \bar{a})$. Add an $n$-tuple of constant symbols $\bar{c}$ to the language of $(\mathcal{A}, \bar{a})$, and let $S=\operatorname{Th}(\mathcal{A}, \bar{a}) \cup\{\varphi(\bar{c})$ : $\varphi(\bar{x}) \in p\}$. It is well-known that Lindenbaum's Lemma, which implies that $S$ has a completion $T$, is provable in $\mathrm{WKL}_{0}$ (see for instance [47]). Then $\{\varphi(\bar{x}): \varphi(\bar{c}) \in T\}$ is a complete type containing $p$.

In the other direction, we argue in $\mathrm{RCA}_{0}$ as follows. Assume that every saturated model is strongly saturated. Let $f$ and $g$ be functions with disjoint ranges, and let $\mathcal{T}$ be a tree such that $P$ is an infinite path of $\mathcal{T}$ if and only if $n \in \operatorname{rng} f \Rightarrow P(n)=1$ and $n \in \operatorname{rng} g \Rightarrow P(n)=0$. To show that $W_{K L}$ holds, it is enough to show that a tree of this form has an infinite path (see [47]). Let $S$ be the set of terminal nodes of $\mathcal{T}$, and let $\widehat{\mathcal{T}}=\mathcal{T} \cup\left\{\sigma 0^{n}: \sigma \in S, n \in \mathbb{N}\right\}$. Let $T=T(\widehat{\mathcal{T}})$ be as in Definition 3.5, and let $\mathcal{A}$ be a model of $T$ (which exists because the Completeness Theorem for theories holds in $\left.\mathrm{RCA}_{0}\right)$. If $\mathcal{T}$ has an infinite path then we are done, so assume otherwise. Let $\bar{a} \subset A$ and let $p\left(x_{0}, \ldots, x_{n-1}\right)$ be a complete $n$-type of $\operatorname{Th}(\mathcal{A}, \bar{a})$. For each $i<n$, the induced 1-type on $x_{i}$ corresponds to an infinite path of $\widehat{\mathcal{T}}$, which must be of the form $\sigma_{i} 0^{\mathbb{N}}$ with $\sigma_{i} \in S$. Since $S$ is a set, we can form the tuple $\left(\sigma_{0}, \ldots, \sigma_{n-1}\right)$, from which we can define a formula generating $p$. So $p$ is principal, and hence realized in $\mathcal{A}$.

Thus $\mathcal{A}$ is saturated and hence, by hypothesis, strongly saturated. Using the notation of Definition 3.5, let $\varphi_{n, s}(x) \equiv P_{n}(x) \wedge P_{n}(x) \wedge \cdots \wedge P_{n}(x)$, where $P_{n}(x)$ is repeated $s+1$ many times, and let $\psi_{n, s}(x) \equiv \neg P_{n}(x) \wedge \neg P_{n}(x) \wedge \cdots \wedge \neg P_{n}(x)$, where $\neg P_{n}(x)$ is repeated $s+1$ many times. Let $\Gamma=\left\{\varphi_{n, s}: f(s)=n\right\} \cup\left\{\psi_{n, s}: g(s)=n\right\}$. Then $\Gamma$ is a partial 1-type of $T$, and hence is realized by some $a \in \mathcal{A}$. It follows that $\left\{n: \mathcal{A} \vDash P_{n}(a)\right\}$ is a path on $\mathcal{T}$. 



\section{Bibliography}

[1] C. J. Ash and J. F. Knight, Computable Structures and the Hyperarithmetical Hierarchy, 1st edn., Stud. Logic Found. Math., vol. 144, Amsterdam, 2000.

[2] D. Belanger, Reverse mathematics of first order theories with finitely many models, J. Symbolic Logic, vol. 79 (2014), 955-984.

[3], $\mathrm{WKL}_{0}$ and induction principles in model theory, Ann. Pure Appl. Logic, vol. 166 (2015), 767-799.

[4] C. C. Chang and H. J. Keisler, Model Theory, 3rd edn., Stud. Logic Found. Math., vol. 73, North-Holland, Amsterdam, 1990 [1st edn. 1973, 2nd edn. 1977].

[5] P. A. Cholak, C. G. Jockusch, Jr. and T. A. Slaman, On the strength of Ramsey's Theorem for pairs, J. Symbolic Logic, vol. 66 (2001), 1-55.

[6] C. J. Conidis, Classifying model-theoretic properties, J. Symbolic Logic, vol. 73 (2008), 885905.

[7] B. F. Csima, Degree spectra of prime models, J. Symbolic Logic, vol. 69 (2004), 430-442.

[8] B. F. Csima, V. S. Harizanov, D. R. Hirschfeldt, and R. I. Soare, Bounding homogeneous models, J. Symbolic Logic, vol. 72 (2007), 305-323.

[9] B. F. Csima, D. R. Hirschfeldt, J. F. Knight, and R. I. Soare, Bounding prime models, J. Symbolic Logic, vol. 69 (2004), 1117-1142.

[10] F. G. Dorais, D. D. Dzhafarov, J. L. Hirst, J. R. Mileti, and P. Shafer, On uniform relationships between combinatorial problems, Trans. Amer. Math. Soc., to appear.

[11] H. Friedman, Subsystems of Set Theory and Analysis, PhD Dissertation, M.I.T., 1967.

[12] _ Higher set theory and mathematical practice, Ann. Math. Logic, vol. 2 (1971), 325357.

[13] Some systems of second order arithmetic and their use, in Proceedings of the International Congress of Mathematicians, Vancouver 1974, vol. 1, Canadian Mathematical Congress, 1975, 235-242.

[14] H. Friedman, S. G. Simpson, and X. Yu, Periodic points and subsystems of second-order arithmetic, Ann. Pure Appl. Logic, vol. 62 (1993), 51-64.

[15] S. S. Goncharov, Strong constructivizability of homogeneous models (Russian), Algebra i Logika, vol. 17 (1978), 363-388, 490; [translated in: Algebra and Logic, vol. 17 (1978), 247263].

[16] S. S. Goncharov and A. T. Nurtazin, Constructive models of complete decidable theories, Algebra i Logika, vol. 12 (1973), 125-42, 243; [translated in: Algebra and Logic, vol 12 (1973), 67-77].

[17] P. Hájek and P. Pudlák, Metamathematics of First-order Arithmetic, second printing, Perspect. Math. Logic, Springer-Verlag, Berlin, 1998.

[18] V. S. Harizanov, Pure computable model theory, in Handbook of Recursive Mathematics (Yu. L. Ershov, S. S. Goncharov, A. Nerode, J. B. Remmel, eds.), Stud. Logic Found. Math., vol. 138-139, Elsevier Science, Amsterdam, 1998, 3-114.

[19] L. Harrington, Recursively presentable prime models, J. Symbolic Logic, vol. 39 (1974), 305309 .

[20] D. R. Hirschfeldt, Slicing the Truth: On the Computable and Reverse Mathematics of Combinatorial Principles, Lecture Notes Series, Institute for Mathematical Sciences, National University of Singapore, vol. 28, World Scientific, Hackensack, NJ, 2015.

[21] D. R. Hirschfeldt and C. G. Jockusch, Jr., On notions of computability theoretic reduction between $\Pi_{2}^{1}$ principles, to appear.

[22] D. R. Hirschfeldt and R. A. Shore, Combinatorial principles weaker than Ramsey's theorem for pairs, J. Symbolic Logic, vol. 72 (2007), 171-206. 
[23] D. R. Hirschfeldt, R. A. Shore, and T. A. Slaman, The atomic model theorem and type omitting, Trans. Amer. Math. Soc., vol. 361 (2009), 5805-5837.

[24] J. L. Hirst, Combinatorics in Subsystems of Second Order Arithmetic, PhD Dissertation, The Pennsylvania State University, 1987.

[25] U. Kohlenbach, Higher order reverse mathematics, in Reverse mathematics 2001 (S. G. Simpson, ed.), Lect. Notes Log., vol. 21, Association for Symbolic Logic and A. K. Peters, Wellesley, MA, 2005, 281-295.

[26] K. M. Lange, The Computational Complexity of Homogeneous Models, PhD Dissertation, The University of Chicago, 2008.

[27] _ The degree spectra of homogeneous models, J. Symbolic Logic, vol. 73 (2008), 10091028.

[28] _ A characterization of the 0-basis homogeneous bounding degrees, J. Symbolic Logic, vol. 75 (2010), 971-995.

[29] K. M. Lange and R. I. Soare, Computability of homogeneous models, Notre Dame J. Formal Logic, vol. 48 (2007), 143-170.

[30] D. Marker, Model Theory: An Introduction, Grad. Texts in Math., vol. 277, Springer, New York, 2002

[31] T. S. Millar, Homogeneous models and decidability, Pacific J. Math, vol. 91 (1980), 407-418

[32] A. Montalbán, Indecomposable linear orderings and hyperarithmetic analysis, J. Math. Logic, vol. 6 (2006), 89-120.

[33] $71-99$

[34] $\ldots$, Open questions in reverse mathematics, Bull. Symbolic Logic, vol. 17 (2011), 431454 .

[35] A. Montalbán and R. A. Shore, The strength of Turing determinacy in second order arithmetic, to appear in Fund. Math.

[36] M. Morley, Decidable models, Israel J. Math., vol. 25 (1976), 233-240.

[37] M. E. Mytilinaios, Finite injury and $\Sigma_{1}$-induction, J. Symbolic Logic, vol. 54 (1989), 212-221.

[38] I. Neeman, The strength of Jullien's indecomposability theorem, J. Math. Logic, vol. 8 (2008), 93-119.

[39] , Necessary use of $\Sigma_{1}^{1}$ induction in a reversal, J. Symbolic Logic, vol. 76 (2011), 561574.

[40] J. B. Paris and L. A. S. Kirby, $\Sigma_{n}$-collection schemas in arithmetic, in Logic Colloquium '7r7, Stud. Logic Found. Math., vol. 96, North-Holland, Amsterdam-New York, 1978, 199-209.

[41] M. G. Peretyat'kin, A criterion for strong constructivizability of a homogeneous model (Russian), Algebra i Logika, vol. 17 (1978), 436-454, 491; [translated in: Algebra and Logic, vol. 19 (1980), 202-229].

[42] D. Seetapun and T. A. Slaman, On the strength of Ramsey's Theorem, Notre Dame J. Forma Logic, vol. 36 (1995), 570-582.

[43] R. A. Shore, Splitting an $\alpha$-recursively enumerable set, Trans. Amer. Math. Soc., vol. 204 (1975), 65-77.

[44] Reverse mathematics: the playground of logic, Bull. Symbolic Logic, vol. 16 (2010), $378-402$.

[45] - Reverse mathematics, countable and uncountable: a computational approach, in Effective Mathematics of the Uncountable (N. Greenberg, J. D. Hamkins, D. R. Hirschfeldt, and R. Miller, eds.), Lect. Notes Log., Association for Symbolic Logic and Cambridge University Press, New York, 2013, 150-163.

[46] S. G. Simpson, Subsystems of Second Order Arithmetic, Perspect. Math. Logic, SpringerVerlag, Berlin, 1999.

[47] Subsystems of Second Order Arithmetic, 2nd edn., Perspectives in Logic, Association for Symbolic Logic and Cambridge University Press, New York, 2009.

[48] T. A. Slaman, $\Sigma_{n}$-bounding and $\Delta_{n}$-induction, Proc. Amer. Math. Soc., vol. $132(2004)$ 2449-2456. 